\title{
Ambient Laser Ablation Electrospray Ionization Mass Spectrometry Imaging
}

\author{
Freddie A. M. G. van Geenen
}





\section{Ambient Laser Ablation Electrospray Ionization Mass Spectrometry Imaging}

Freddie A. M. G. van Geenen 


\section{Thesis committee}

\section{Promotors}

Prof. Dr M. W. F. Nielen

Professor of Analytical Chemistry

Wageningen University \& Research

Prof. Dr H. Zuilhof

Professor of Organic Chemistry

Wageningen University \& Research

\section{Co-promotor}

Dr M. C. R. Franssen

Associate professor at the Laboratory of Organic Chemistry

Wageningen University \& Research

\section{Other members}

Prof. Dr H. A. Schols, Wageningen University \& Research

Prof. Dr R. M. A. Heeren, Maastricht University

Prof. Dr E. J. R. Sudhölter, Delft University of Technology

Prof. Dr G. W. Somsen, Vrije Universiteit Amsterdam

This research was conducted under the auspices of the Graduate School VLAG (Advanced studies in Food Technology, Agrobiotechnology, Nutrition and Health Sciences). 


\section{Ambient Laser Ablation Electrospray Ionization Mass Spectrometry Imaging}

Freddie A. M. G. van Geenen

Thesis

Submitted in fulfilment of the requirements for the degree of doctor

at Wageningen University

by the authority of the Rector Magnificus

Prof. Dr A. P. J. Mol,

in the presence of the

Thesis Committee appointed by the Academic Board

to be defended in public

on Wednesday, July $3^{\text {rd }}$

at 4 p.m. in the Aula. 
Freddie A. M. G. van Geenen

Ambient Laser Ablation Electrospray Ionization Mass Spectrometry Imaging 164 pages

PhD thesis, Wageningen University, Wageningen, The Netherlands (2019)

With references, with summary in English

ISBN 978-94-6343-922-0

DOI $\quad 10.18174 / 473023$ 


\section{To my family}





\section{Table of contents}

$\begin{array}{lr}\text { List of Abbreviations } & 9\end{array}$

$\begin{array}{ll}\text { Chapter 1: General Introduction } & 11\end{array}$

Chapter 2: Ambient Characterization of Synthetic Fibers by Laser Ablation Electrospray Ionization Mass Spectrometry

Chapter 3: Reactive Laser Ablation Electrospray Ionization TimeResolved Mass Spectrometry of Click Reactions

Chapter 4: $\mathrm{TiO}_{2}$ Photocatalyzed Oxidation of Drugs Studied by Laser Ablation Electrospray Ionization Mass Spectrometry

Chapter 5: Laser Ablation Electrospray Ionization Hydrogen/Deuterium Exchange for Structure Elucidation in Ambient Mass Spectrometry Imaging

Chapter 6: General Discussion and Future Perspectives

Summary

Acknowledgements

Curriculum Vitae

List of Publications 



\section{List of Abbreviations}

\begin{tabular}{|c|c|}
\hline $\mathrm{ACN}$ & acetonitrile \\
\hline $\mathrm{BCN}$ & bicyclo[6.1.0]non-4-yn \\
\hline $\mathrm{Cl}$ & chemical ionization \\
\hline DART & direct analysis in real time \\
\hline DESI & desorption electrospray ionization \\
\hline EIC & extracted ion current \\
\hline ELDI & electrospray-assisted laser desorption ionization \\
\hline ESI & electrospray ionization \\
\hline FA & formic acid \\
\hline FA-APGD & flowing afterglow atmospheric pressure glow discharge \\
\hline HDX & hydrogen/deuterium exchange \\
\hline $\mathrm{H}_{2} \mathrm{O}$ & ultrapure water \\
\hline IEDDA & inverse electron demand Diels-Alder addition \\
\hline IMS & ion mobility separation \\
\hline IR-MALDESI & infrared matrix-assisted laser desorption electrospray ionization \\
\hline LA & laser ablation \\
\hline LAESI & laser ablation electrospray ionization \\
\hline Leu-enk & leucine-enkephalin \\
\hline M5 & $\begin{array}{l}\text { poly[2,6-diimidazo }\left(4,5-\beta-4^{\prime}, 5^{\prime}-\varepsilon\right) \text { pyridinylene-1,4-(2,5- } \\
\text { dihydroxy)phenylene] }\end{array}$ \\
\hline MALDI & matrix-assisted laser desorption ionization \\
\hline $\mathrm{MeOH}$ & methanol \\
\hline MS & mass spectrometry \\
\hline MSI & mass spectrometry imaging \\
\hline PA & polyamide \\
\hline PADI & plasma-assisted desorption ionization \\
\hline PEG & polyethylene glycol \\
\hline PET & polyethylene terephthalate \\
\hline PMMA & poly(methyl methacrylate) \\
\hline PPG & polypropylene glycol \\
\hline PMS & poly(a-methyl styrene) \\
\hline SARM & selective androgen receptor modulator \\
\hline $\mathrm{SI}$ & supporting information \\
\hline SIMS & secondary ion mass spectrometry \\
\hline TA-APGD & thermal-assisted atmospheric pressure glow discharge \\
\hline TIC & total ion current \\
\hline TOF & time of flight \\
\hline TRMS & time-resolved mass spectrometry \\
\hline TWIM & travelling wave ion mobility \\
\hline
\end{tabular}


Chapter 1

\section{General Introduction}


Chapter 1 


\section{Imaging}

An image is a product that depicts a concept or visual perception of a subject, like a picture or painting. Images are captured by, e.g., optical devices such as cameras or biological organs like eyes. Images can be reconstructed or reproduced by drawing, painting, printing, or projecting the perception onto a material or screen. Images are highly valuable and stated "to be worth a thousand words." This statement is a reference to the often high information density, comprising countless details, presented in a clear and straightforward fashion. Most images today are photographs, which are made with camera's and capture subjects in spacetime. The same method is often used by many scientists and specialists to record, study and present their samples. When the relevant information is located on the microscopic scale, like in cells or their clusters, lenses are used to enlarge the images for their detailed study. The knowledge obtained in such a setting is limited to the information that is carried by and contained within the recorded light, such as color and spatial arrangement. For example, an image of a leaf is sufficient to identify the sample as a leaf, yet there is much more information within this leaf that cannot be accessed visually. Detailed information on (bio)molecules that are present within the leaf, like carbohydrate structures, metabolites, proteins, or administered pesticides is missing in optical images. With access to such information, biosynthesis areas, transport pathways, and storage locations, as well as immune or metabolism responses to stimulations, could all be monitored long before changes can be observed at the phenotypical level. The metabolism response of a leaf after infection by Plasmopara viticola is shown in Figure 1 by optical imaging and molecular imaging (fluorescence and mass spectrometry) techniques. In these images, an infection on the upper zone of the leaf is visible when measured with molecular imaging techniques, whereas no differences between the infected and the control area in the optical image are observed. Hence, molecular imaging enables the visualization, characterization, and measurement of biological processes, leading to a more detailed understanding of samples in their totality.

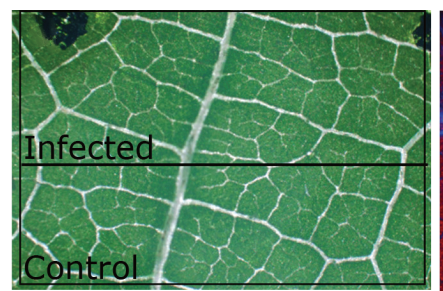

Optical image

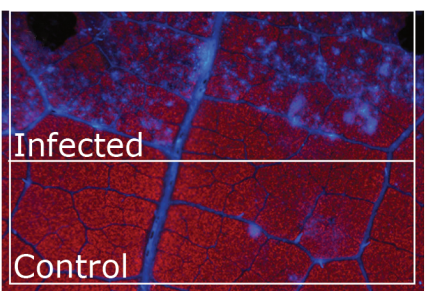

Fluorescence image

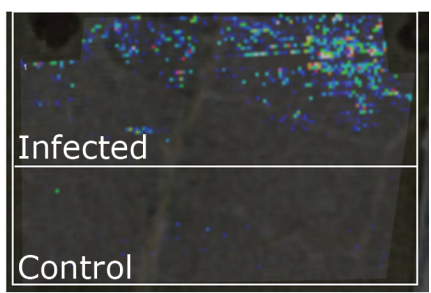

Mass spectrometry image

Figure 1. Optical, fluorescence and mass spectrometry images of the same area on a leaf. The horizontal line shows the infected area (upper zone) and the control area (lower zone). Image was reprinted from ${ }^{1}$, with permission from the American Chemical Society. 


\section{Molecular Imaging}

Many insightful observations on complex molecular processes in living cells, tissues and animals by molecular imaging platforms have already greatly advanced our understanding of biological systems. ${ }^{2,3}$ Several complementary molecular imaging techniques, including positron-emission tomography (PET), ${ }^{4}$ fluorescence (along with bioluminescence and phosphorescence) imaging and nuclear magnetic resonance imaging (MRI), 5,6 can image different parameters of cellular function. In PET imaging, a radioactive probe is used to follow analytes of interest. This probe (which is based on an unstable isotope) can be incorporated in a new chemical entity to follow the location of the probe and its metabolites in ADME (absorption, distribution, metabolism, and excretion) studies, or attached to a biomolecule -like an amino acid or carbohydrate- to follow biosynthesis routes and local uptake. PET is a quantitative technique and allows the monitoring of relative changes over time, e.g., as a result of stimuli responses or disease progression. Additionally, PET provides excellent sensitivity to observe analytes even in the nanomolar range and can image with a spatial resolution of approximately a few $\mathrm{mm} .^{7,8}$ The main disadvantages of this technique are the expensive on-site synthesis of radioactive probes, the radioactivity of the probe and the fact that only the signal of the probe can be imaged. Hence, there is no differentiation between the labelled molecule and its metabolites, and any metabolism products without the probe remain unnoticed. Although PET is excellent for the imaging of single molecules, it provides little information on their interactions. Different approaches, including fluorescence imaging, are required to study those (bio)molecular interactions.

Fluorescence imaging is a probe-based method too, exploiting rigid molecular structures that can significantly emit photons upon excitation by electromagnetic radiation instead of utilizing the radiation of an unstable atomic isotope. These probes consequently eliminate the drawbacks of radioactivity and the requirement for expensive on-site synthesis. They are however often unsuitable for incorporation in new chemical entities due to a much larger size, possibly disturbing metabolism by hampering selective protein interactions. These probes are most frequently designed for coupling with a biomolecule of interest, in that way visualizing sophisticated processes including protein complexation, motility, and metabolism. ${ }^{9}$ Figure 2 shows a time-resolved fluorescence image of a tumor (encircled in red) bearing mice after injection with a probe that binds to tumor cells. Fluorescence imaging is regarded as a technique that provides complementary information that cannot be obtained with PET, like information contained within single cells or molecular interactions. Major drawbacks of fluorescence imaging techniques however are its need for fluorescent probes as well as a low tissue penetration depth. Nonetheless, the complementary information from the focus on interactions, an excellent sub- $\mu \mathrm{m}$ spatial resolution, and micromolar sensitivity ensures that fluorescence imaging is widely established for the imaging of analytes and/or their molecular interactions for superficial targets. PET and fluorescence imaging are thus mostly 
complementary techniques, each providing a partial understanding of the total biological system.

An alternative molecular imaging approach is $\mathrm{MRI},{ }^{10}$ which is commonly known for its analysis of the human body. The MRI signal is obtained by the alignment of endogenous nuclei with spins -like ${ }^{1} \mathrm{H},{ }^{13} \mathrm{C},{ }^{19} \mathrm{~F}$ - in a sample within a strong magnetic field, subsequent perturbation by a radio-frequency pulse, and detection of the relaxation signals. ${ }^{11-13} \mathrm{MRI}$, like PET, is not significantly limited by penetration depth and comprises a spatial resolution of approximately $0.1 \mathrm{~mm}$. However, its very poor sensitivity is a major disadvantage. Additionally, the detection and identification of specific analytes in the presence of all endogenous cellular molecules is a daunting task. Therefore, probes were also introduced for MRI. These probes are generally designed to interact with target molecules and dramatically change the relaxation properties of protons in surrounding water molecules, improving selectivity and sensitivity. ${ }^{14} \mathrm{MRI}$ is thus capable of detecting biomolecules and/or their interactions noninvasively and deep within samples.

The major disadvantages of all "probe-based" approaches are the need to identify the molecules of interest prior to the experiment to apply an appropriate label, and that all information obtained is only about these specific molecules, interactions, and pathways. In order to obtain a full understanding of the biological system all separate information is required to be combined and integrated into an underlying biological framework. While these approaches are and will always be important, many biological and clinical problems can only be solved with a "complete" or "systems" approach. The development of molecular imaging techniques that can directly measure multiplex molecular interactions and pathway connectivity is thus of absolute importance to advance the knowledge of biological systems in their totality.

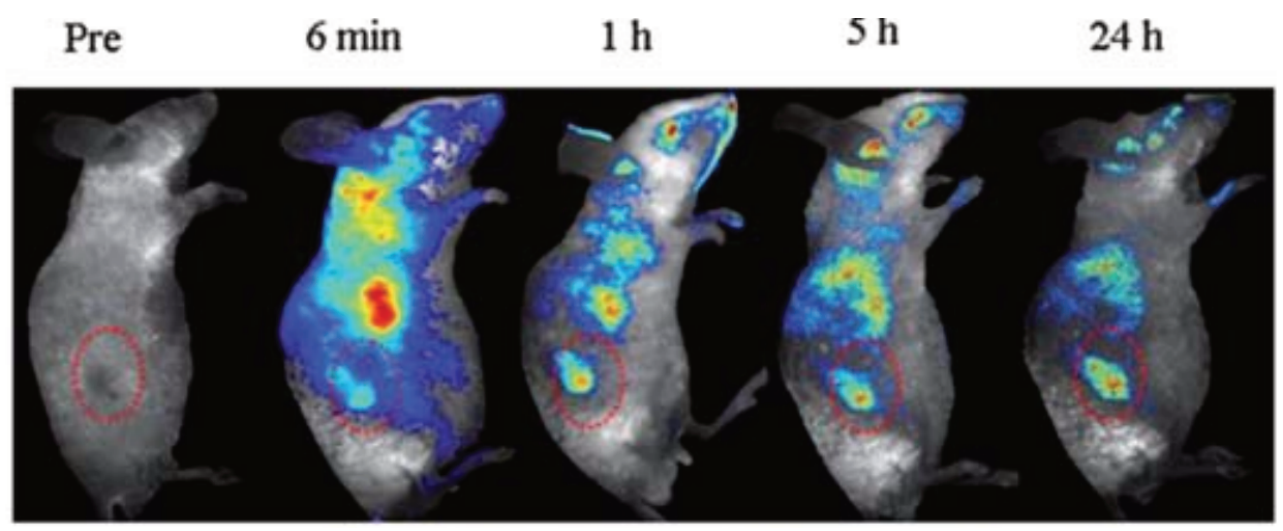

Figure 2. Time-resolved in vivo fluorescence imaging of a tumor bearing mice after tail injection of a fluorescent probe. Image was reprinted from ${ }^{15}$, with permission from The Royal Society of Chemistry. 


\section{Molecular Imaging by Mass Spectrometry}

Mass spectrometry imaging (MSI) is a molecular imaging technique capable of simultaneously measuring numerous molecules with exceptional molecular specificity. ${ }^{16,17}$ $\mathrm{MSI}$ is label free and can create molecular images of sample surfaces, like a biopsy or an entire animal tissue section. MSI therefore has great potential for disease diagnosis, ${ }^{18}$ following disease progression, ${ }^{19}$ as well as to observe how a drug and its metabolites distribute. ${ }^{20,21}$ Besides biological samples, MSI can also be used in material sciences to obtain information on surfaces, such as modifications and defects. ${ }^{22,23}$ The potential and continuous development of $\mathrm{MSI}$ has resulted in a substantially increased popularity over the last two decades, which can be seen from the amount of publications on MSI since 1997 as depicted in Figure 3. However, there is still much room for improving sensitivity, spatial resolution, quantification aspects and structure elucidation. ${ }^{24}$

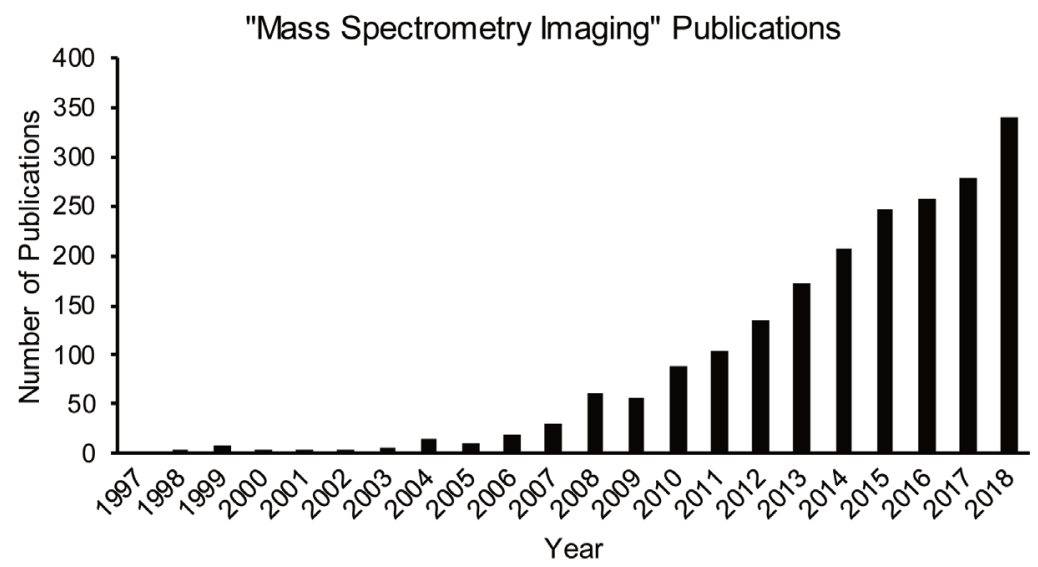

Figure 3. Number of publications on "Mass Spectrometry Imaging" per year based on a SciFinder ${ }^{\circledR}$ search.

\section{Mass Spectrometry}

Mass spectrometry (MS) is an analytical technique that is suitable for qualitative and quantitative analysis of samples. MS analysis is achieved by ionization of molecules and subsequent detection of generated gas phase ions based on their mass-to-charge $(\mathrm{m} / \mathrm{z})$ ratio. The separation of ions on $\mathrm{m} / \mathrm{z}$ is accomplished by exposing them to an electric or magnetic field. Various mass analyzers are available to perform the separation, each comprising distinctive characteristics. In classical magnetic sector instruments, ions with various $\mathrm{m} / \mathrm{z}$ values undergo a different degree of deflection, bending the trajectories of the ions as they pass through the analyzer. Magnetic sector instruments are used to continuously measure a narrow $m / z$ range at sub-ppm mass accuracy. ${ }^{25-27}$ Quadrupole instruments use oscillating electric fields to selectively stabilize the paths of ions passing through the parallel quadrupole 
rods. The potential on the rods can be altered rapidly, allowing for a stable trajectory for a wide range of $\mathrm{m} / \mathrm{z}$ values in a sequential process. The mass accuracy is at best $20 \mathrm{ppm}$, but quadrupole instruments are versatile and are often combined with other mass analyzers for tandem MS experiments. ${ }^{28}$ lon trap instruments measure the orbital frequency of electrostatically trapped ions in space. Their mass accuracy is similar to quadrupole instruments, but ion traps can fragment ions in time, producing additional structural information. ${ }^{29}$ The orbital frequency of trapped ions can also be measured by trapping them in a magnetic field, like with Fourier transform ion cyclotron MS (FT-ICR) instruments. FT-ICR instruments comprise, like sector instruments, a sub-ppm mass accuracy, but as a trap based instrument, it has a slow scan rate. ${ }^{30}$ Orbitrap instruments trap ions in orbit around a central electrode; endcap electrodes are used for ion oscillation along the trapped axis, allowing the accurate measurement of the frequency of the ions along this axis. Orbitraps provide sub-ppm mass accuracy and have increased space charged capacity at higher masses as well as a larger trapping volume compared to FT-ICR instruments. ${ }^{31}$ Finally, time-of-flight (TOF) instruments measure the velocity of ions after subjecting them to an accelerating field. . $^{27,32,33}$ TOF analyzers typically achieve 2-5 ppm mass accuracy, which allows for assessment of molecular formulas. ${ }^{34}$ Additionally, a rapid scan rate for the simultaneous measurement of a broad $m / z$ range is a distinctive aspect for TOF mass analyzers.

After ion separation on $\mathrm{m} / \mathrm{z}$ their amount is determined by the detector, which is usually a counting device for charged species, like an electron multiplier tube or a microchannel plate detector. The more ions that reach the detector, the more counts will be recorded. ${ }^{35,36}$ Obtained data of separated ions and their intensities are displayed in a mass spectrum. Figure 4 shows an electrospray ionization mass spectrum of a caffeine solution. Protonated caffeine is detected accordingly to its elemental composition $\left[\mathrm{C}_{8} \mathrm{H}_{10} \mathrm{~N}_{4} \mathrm{O}_{2}+\mathrm{H}\right]^{+}$as $m / z$ value 195.088 .

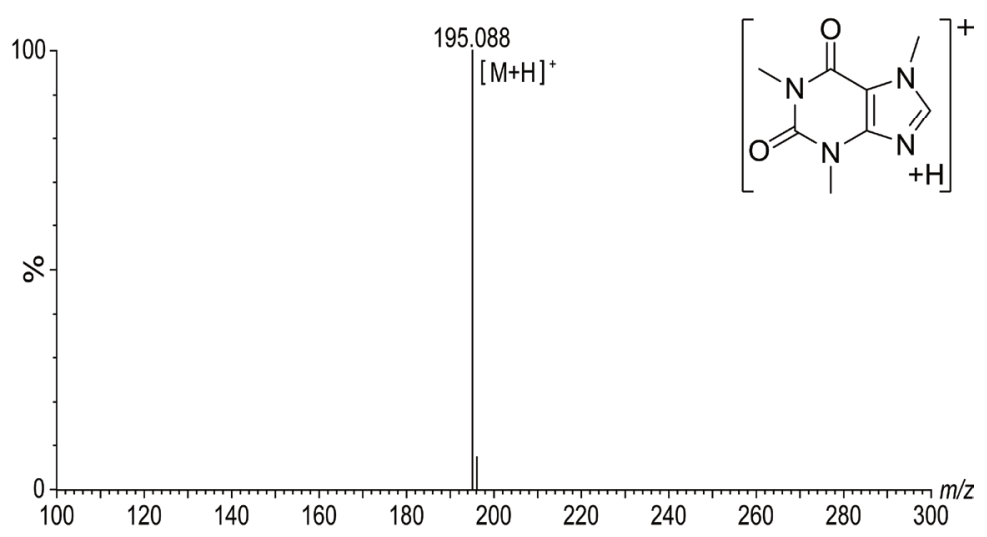

Figure 4. Electrospray ionization mass spectrum and chemical structure of protonated caffeine, detected at $m / z 195.088$. 
As discussed above, mass spectrometry is only able to separate and detect gasphase ions. Any uncharged analytes are therefore required to be charged prior to MS analysis. Apart from classical ionization methods such as electron impact (EI) and chemical ionization $(\mathrm{Cl})$, various methods were developed for the generation of gas-phase ions, like electrospray ionization (ESI) and matrix-assisted laser desorption ionization (MALDI). ${ }^{27,37,38}$ In ESI an analyte solution flows through a metal capillary, and upon application of a high voltage, an electrostatic spray will be created at the capillary tip. ${ }^{39}$ The resulting charged microdroplets can be considered monodisperse and become smaller in size due to solvent evaporation and subsequent droplet fission. ${ }^{40,41}$ In the end, gas-phase ions are generated containing one or multiple charges. These charges are mostly the result of the addition or removal of a proton(s), however also adducts of, e.g., sodium or potassium cations can be observed. ESI is abundantly used for the analysis of compounds with a wide range of molecular weights, starting from small molecules (ca. < $500 \mathrm{Da}$ ) up to very high molecular weight (MDa) nonvolatile compounds such as proteins and their complexes, that can still be measured at relatively low $\mathrm{m} / \mathrm{z}$ ranges due to multiple charges. ${ }^{42-44}$ Along with ESI, MALDI is one of the most important ionization methods for nonvolatile high molecular weight compounds. For instance, peptides, proteins, oligonucleotides, oligosaccharides, and synthetic polymers have been studied with MALDI-MS. ${ }^{45,46}$ In MALDI analytes are most frequently co-crystallized with an excess of matrix material, which absorbs the pulsed laser energy. Analytes are subsequently desorbed/ablated along with the matrix material and are protonated or deprotonated by and within the resulting hot plume of desorbed/ablated material before acceleration into the mass spectrometer. ${ }^{47}$ Small molecules are compatible with MALDI as well, although interfering signals derived from the MALDI matrix and poor reproducibility of signal intensities are main challenges below $\mathrm{m} / \mathrm{z} 1000.48,49$

Additional molecular structural information and/or increased selectivity for analyte quantification can be obtained with tandem mass spectrometry (either in time: $\mathrm{MS}^{n}$, or space: MS/MS). ${ }^{50,51}$ Considering soft ionization techniques, e.g., ESI and MALDI, tandem MS is commonly achieved by precursor ion selection, subsequent fragmentation, and MS analyses of obtained fragments. These fragments contain information on specific substructures derived from the original structure, which assists in the structure elucidation of unknowns. Also, the sequence of building blocks in large molecules such as amino acids in proteins or monomers in polymers can be determined by tandem MS. ${ }^{52,53}$ Furthermore, the selection of a precursor ion together with a specific fragment will often increase selectivity and/or sensitivity of tandem MS due to a substantial reduction of interfering background ions comprising different fragmentation patterns.

Mass spectrometric analysis is generally performed as a continuous measurement in time, recording consecutive MS spectra per time unit until the analysis is completed. The intensity of ions is then commonly provided in a graph versus time as, e.g., total ion current (TIC) for the sum of all ions, or extracted ion current (EIC) for a single $m / z$ value. Because of 
its great utility, structure elucidation capabilities, sensitivity, selectivity, and options for hyphenation with separation techniques like chromatography, electrophoresis, and ion mobility (IMS), MS has developed into a powerful and abundantly used analytical technique.

\section{Mass Spectrometry Imaging}

Mass spectrometry imaging (MSI) enables untargeted investigations into the spatial distribution of analytes. Two approaches were developed for MS image acquisition; the microprobe and microscope modes. ${ }^{16,54}$ Both approaches are depicted in Figure 5 . In the microprobe approach a focused -e.g., laser/ion/charged droplet- beam is fired at a single, spatially defined spot on the sample. ${ }^{55}$ The coordinates of this spot along with the resulting mass spectrum are stored. Then the next spot will be targeted and another mass spectrum is recorded. This process is repeated in a step by step approach until the entire sample area has been analyzed. The molecular image can then be reconstructed from the spatially correlated mass spectra. In this approach, the spatial resolution of the image is corresponding with the focused beam spot size. Hence, a smaller spot size results in improved spatial resolution, but at the cost of decreased molecular information (due to a lower sensitivity as less sample material is injected) and longer analysis time (as a result of increased number of sample spot locations). The microprobe approach is the most abundantly used method, featuring wide compatibility with various ion sources and mass analyzers.

In the microscope approach, a large unfocused -laser/ion- beam is directed at a spatially defined area on the sample. ${ }^{56-60}$ Sample material from this area is desorbed simultaneously and is recorded by a position-sensitive detection system (e.g., a specially modified microchannel plate), magnifying the image while retaining spatial information. This approach results in improved spatial resolution and a substantially reduced analysis time. Although favorable for rapid molecular image generation at high spatial resolution, the molecular information can only be recorded in a linear time-of-flight approach. This results in reduced molecular specificity as well as limited identification capabilities in comparison with microprobe mode studies utilizing high-resolution mass analyzers and/or MS/MS approaches. 

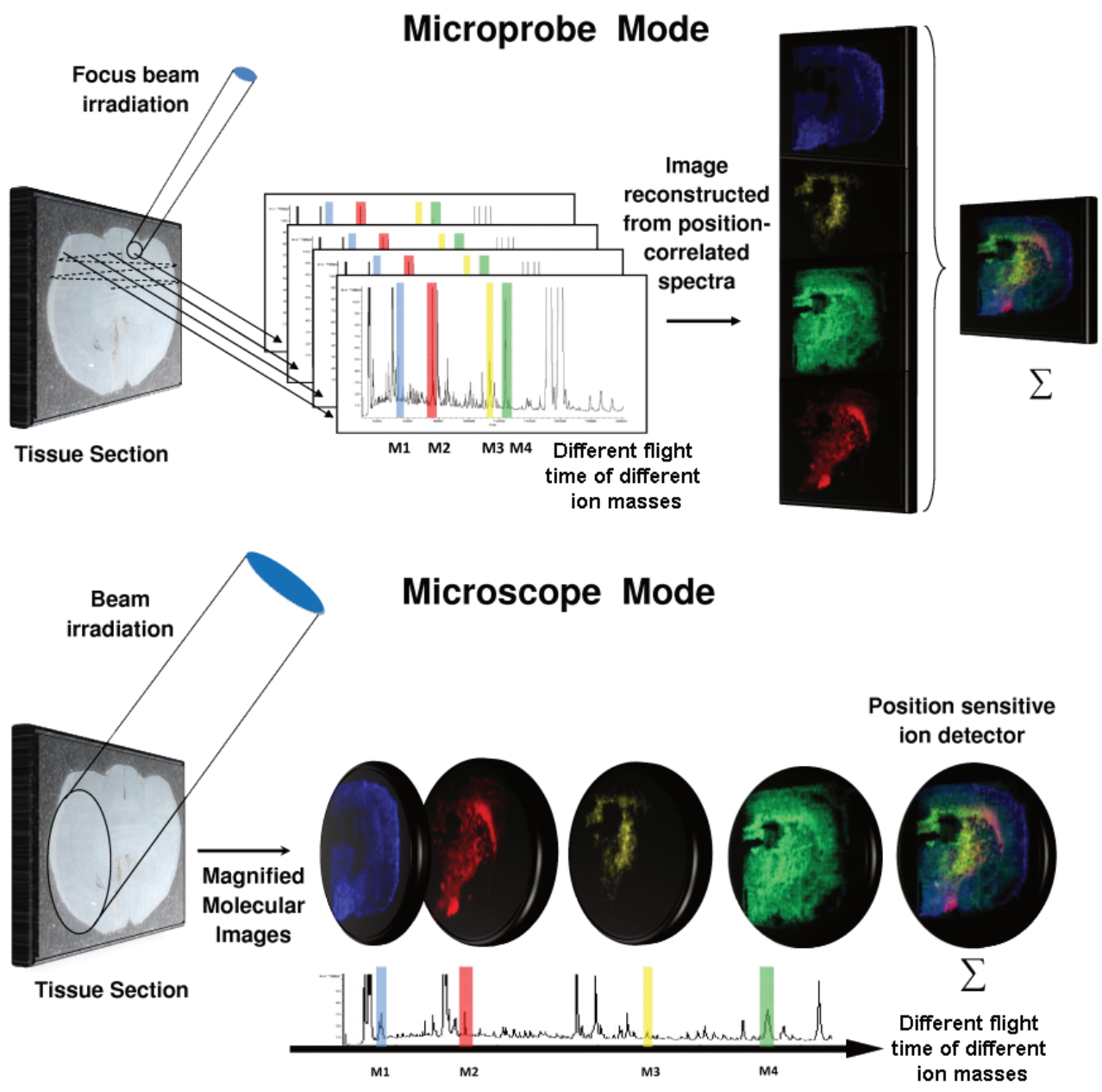

Figure 5. Image reconstruction from spatial coordinates and corresponding mass spectra. Image was reprinted from ${ }^{61}$, with permission from John Wiley and Sons.

Aside from the microprobe and microscope approaches, MSI is typically a four-step process and involves sample pretreatment, desorption and ionization, mass analysis, and data processing. Most frequently biological samples are prepared (e.g., frozen and sectioned) prior to MSI analysis. These samples often require further pretreatment, which is a delicate step and mostly dependent on the subsequent ionization technique. In the perspective of molecular imaging in a "complete" or "systems" approach, essential aspects of sample pretreatment are to prevent the loss of molecular information as well as the delocalization of analytes. The classical and well-established desorption and ionization techniques for MSI are SIMS and MALDI (both methods are shown in Figure 6). SIMS was initially developed for 
imaging of surfaces in the material sciences, like chips and semiconductors in the $1960 \mathrm{~s} .{ }^{62}$ Imaging of biological samples was only introduced at the beginning of the $21^{\text {st }}$ century. ${ }^{55}$ SIMS utilizes a beam of primary ions to bombard a spot on the sample surface, causing substantial molecular fragmentation and producing secondary ions out of surface molecules for MS detection. The sample spot size can be focused to only tens of nanometers, ${ }^{63,64}$ providing exceptional spatial resolution. The substantial fragmentation, in combination with the fact that only a small percentage of the surface molecules are ionized, ${ }^{65}$ limits the experimental mass range to approximately $1000 \mathrm{Da} .{ }^{66}$ SIMS is due to this limitation mostly used for the imaging of elements ( $\mathrm{Na}, \mathrm{K}, \mathrm{Ca}$, etc.) and small molecules (drugs, fatty acids, lipids) in biological samples. ${ }^{55,67-71}$ A lower degree of fragmentation can be obtained by applying a matrix to the surface -to absorb the high energy- or the incorporation of cluster ion sources. ${ }^{72-74}$ These methods substantially increase the sensitivity for molecules up to ca. $5000 \mathrm{Da}$, but also result in reduced spatial resolution due to the size of matrix crystals or ion clusters. ${ }^{75}$

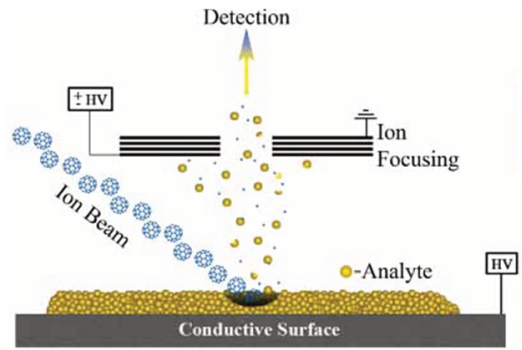

SIMS

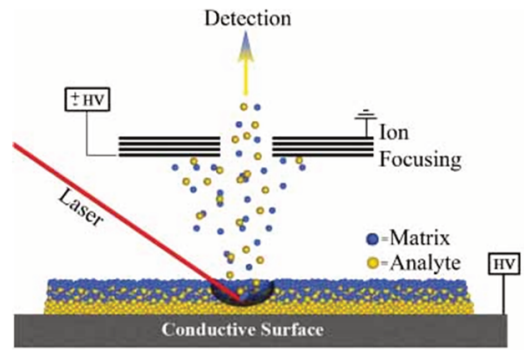

MALDI

Figure 6. Ionization mechanisms for SIMS and MALDI ionization sources. Image was reprinted from ${ }^{76}$, with permission from John Wiley and Sons.

MALDI imaging was first introduced in the late 1990s where it was used for the imaging of peptides and proteins in biological samples. ${ }^{77}$ In MALDI, the sample is covered with a matrix that extracts and incorporates molecules from the surface. A laser, instead of an ion beam, is then utilized to desorb and ionize matrix and sample molecules before MS detection. Although the matrix limits the spatial resolution to ca. 10-20 $\mu \mathrm{m}$, i.e., the size of matrix crystals, ${ }^{55,78}$ it drastically increases ionization efficiency for the analysis of molecules up to $200 \mathrm{kDa}$. MALDI causes little molecular fragmentation and provides high sensitivity in a wide mass range, which established MALDI as the leading method for peptide and protein MSI. ${ }^{16,76,79}$ Sample pretreatment is however also a limiting factor in MALDI (and SIMS) imaging. The application of a matrix prevents real-time analysis and can substantially affect the outcome of MSI, such as analyte losses and the delocalization of analytes. ${ }^{80,81}$ Cells will react to their environment and the often acidic and denaturing matrices can cause problems preserving intact protein configurations and complexes. The type of matrix material and its application process are also crucial parameters for obtaining high quality and reproducible 
images, making it challenging for non-specialist users from other disciplines in biology and medicine. ${ }^{82-84}$ Image quality can also be affected by vacuum conditions in the MALDI or SIMS ion source and sample stage, disrupting or damaging biological samples when exposed. ${ }^{85,86}$ Additionally, in case of combining MSI with other molecular imaging techniques (multimodal molecular imaging), both the matrix and vacuum conditions can cause several different challenges, ranging from matrix interferences to sample disruption. The investigating of samples in their native conditions would thus be a valuable development for MSI analysis.

\section{Ambient Mass Spectrometry Imaging}

Ambient mass spectrometry was introduced in 2004 to record mass spectra of samples under ambient conditions, without any sample pretreatment. ${ }^{87,88}$ Although ion transmission is reduced in comparison with vacuum approaches, a wide variety of ambient MS techniques was developed due to the importance of native analysis for biological samples. Ambient MS techniques follow three main principles: 1) ions are generated under ambient conditions before introduction into a mass spectrometer, 2) samples do not require any pretreatment, and 3) analytes are directly desorbed from the sample surface. Ambient techniques can be arranged according to their general ionization process, which is mostly based on the use of either an excited gas or charged solvent droplets. These groups are divided into $\mathrm{Cl}$-gasrelated techniques, including ambient solid analysis probe (ASAP), ${ }^{89}$ direct analysis in real time (DART), ${ }^{88}$ desorption atmospheric pressure chemical ionization (DAPCI), ${ }^{90}$ dielectric barrier discharge ionization (DBDI), ${ }^{91}$ and plasma-assisted desorption/ionization (PADI), ${ }^{92}$ as well as ESI -solvent- related techniques, such as desorption electrospray ionization (DESI), ${ }^{93}$ desorption sonic spray ionization (DeSSI), ${ }^{94}$ electrospray laser desorption/ionization (ELDI), ${ }^{95}$ laser ablation electrospray ionization (LAESI), ${ }^{96}$ and neutral desorption extractive electrospray ionization (ND-EESI). ${ }^{97}$ An overview of ambient MS techniques is depicted in Figure 7.

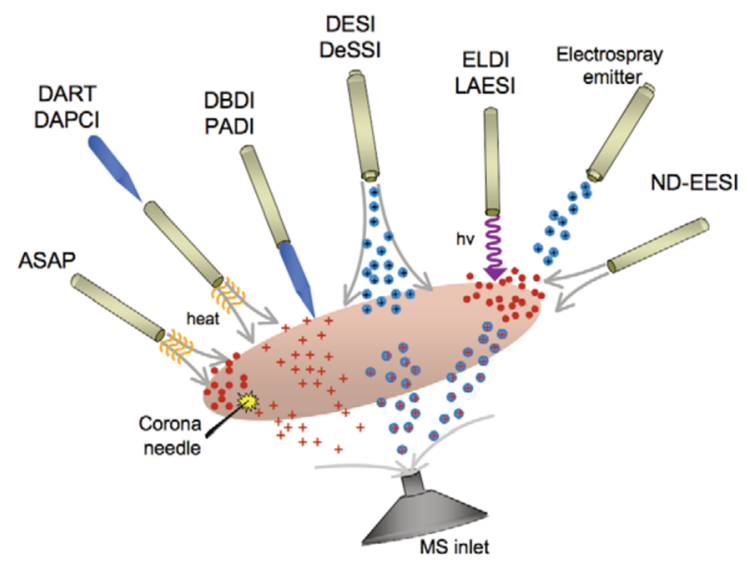

Figure 7. Techniques used in ambient desorption ionization. Image was reprinted from, ${ }^{98}$ with permission from Elsevier. 
The technique widely established for ambient MSI is DESI. ${ }^{93} \mathrm{DESI}$ is an adaptation of ESI and focusses a beam of charged ESI droplets onto the sample surface (depicted in Figure 8). Analytes are extracted by accumulated solvent on the surface, and subsequent collisions of ESI droplets with the wetted surface desorb secondary droplets with incorporated analytes for mass spectrometric analysis. ${ }^{99-101}$ The mass range for DESI analysis is limited to ca. $2000 \mathrm{Da}$, but DESI utilizes the same ionization mechanisms as ESI, and large molecules like proteins can still be measured as multiple charged ions. ${ }^{102-104}$ Also reagents can be added to the DESI solvent to perform online reactions with surface analytes for structure elucidation or sensitivity enhancements. ${ }^{105-109}$ DESI has a spatial resolution of about $200 \mu \mathrm{m}$, although the wetting of the sample surface can cause diffusion of extracted analytes. DESI requires flat samples, as height differences might cause the ESI needle to scratch the surface.

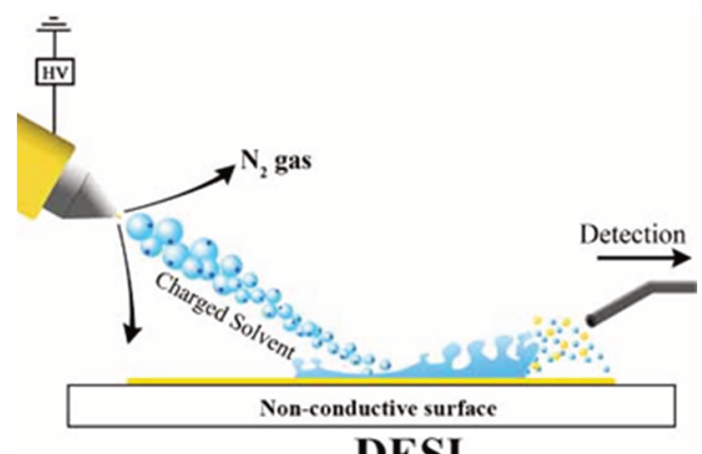

Figure 8. Ionization mechanism for DESI. Image was reprinted from ${ }^{76}$, with permission from John Wiley and Sons.

While MALDI, SIMS, and DESI remain primarily utilized in the field of MSI, new techniques are continuously being developed. These techniques are often improvements on current methods or combinations of existing ionization sources. The endgame in MSI development would be a technique that is simple, rapid, sensitive, quantitative, reproducible, capable of native MSI in real-time, providing sub-cellular spatial resolution and images in a complete/systems approach. As DESI theoretically comprises many of these aspects, it would be interesting to remove some of its limitations. The spatial resolution and incompatibility with non-flat samples can be improved by making use of laser desorption as used in MALDI systems. Instead of a MALDI matrix, the laser energy can be absorbed by endogenous solvent molecules, like water. Desorbed analytes can then be post-ionized with ambient MS techniques. As $\mathrm{Cl}$ based techniques are unsuitable for medium and large compounds, ESI is an excellent post-ionization choice for the next generation of mass spectrometry imaging, due to a considerable mass range coverage thanks to multiple charging and a soft ionization mechanism. 


\section{Ambient Laser Ablation Electrospray lonization Mass Spectrometry Imaging}

Nemes and Vertes first described laser ablation electrospray ionization (LAESI) as a technique for in vivo analysis of biological samples at ambient conditions for MSI. ${ }^{96}$ LAESI exploits a $2940 \mathrm{~nm}$ mid-IR laser to target the $-\mathrm{OH}$ stretch vibration of endogenous water molecules in a sample. Ablated analytes are intercepted by charged microdroplets generated by an orthogonally placed electrospray emitter. Ionization occurs following the soft ESI mechanisms, thus intact molecular ions and adducts are often observed in LAESI mass spectra. ${ }^{110}$ An overview of the LAESI process is presented in Figure 9. The spatial resolution, as well as the sensitivity, are strongly related with the laser spot size; a smaller spot gives a better spatial resolution, whereas a larger spot provides better sensitivity due to more ablated sample material. LAESI MSI is then achieved following the microprobe mode by exploiting the laser $x$-y coordinates.

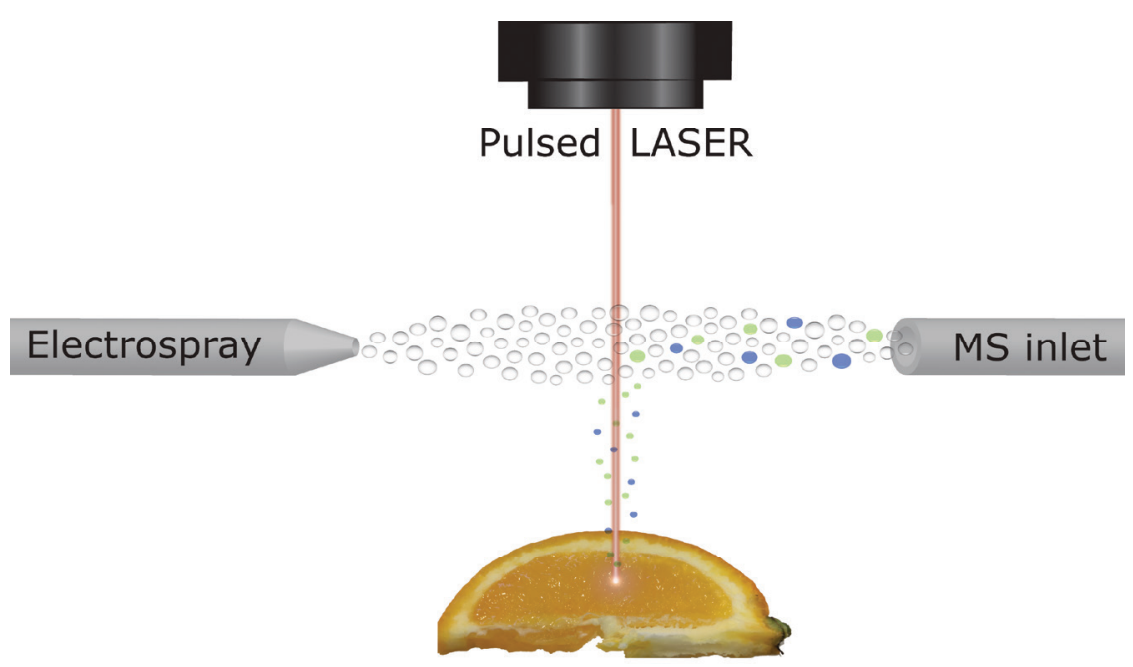

Figure 9. Overview of the LAESI mechanism.

LAESI applicability was successfully demonstrated in the analysis of tissue, food contaminants, and single cells. ${ }^{111-120}$ Yet, there is plenty of potential for LAESI development. The capabilities of LAESI analysis for materials that do not contain endogenous water to absorb laser energy is an exciting field for exploration. Combining LAESI with in-situ chemical reactions, aiming for enhanced sensitivity and selectivity, is an area that is also entirely unexplored. As high-resolution MS and MS/MS methods are frequently insufficient for unambiguous structure elucidation in ambient MSI, the development of additional approaches to confirm and identify biomolecules is of paramount importance in any untargeted study and should thus be investigated for LAESI. A combination of such developments could rapidly increase the scientific and social impact of ambient MSI. 


\section{Aim of this Research}

Molecular imaging provides information of samples on the molecular level, often long before visual changes can be observed. Several molecular imaging techniques are available to study and understand biological tissues and processes, like positron-emission tomography, fluorescence, and magnetic resonance imaging. These techniques however often require chemical probes and thus image in a targeted approach. As many biological questions can only be answered in a systems approach, molecular imaging methods that can simultaneously measure many molecules are desired.

Mass spectrometry imaging is capable of measuring many molecules simultaneously without the use of chemical probes, additionally eliminating the requirement to identify compounds for probe design prior to analysis. Mass spectrometry imaging experiments frequently require vacuum conditions at the sample stage/ion source and extensive sample pretreatment such as matrix application. Vacuum conditions can disrupt or damage biological samples when exposed, and sample pretreatment prevents real-time analyses and can cause analyte losses, analyte delocalization and denaturing of proteins. Ambient ionization was introduced to measure samples under ambient conditions without any sample pretreatment. The ion transmission in ambient mass spectrometry imaging is however decreased in comparison with vacuum approaches, reducing the amount of molecular information.

With the work described in this thesis, we aimed to improve the capabilities and broaden the scope of laser ablation electrospray ionization mass spectrometry imaging. The work is based on laser ablation combined with electrospray ionization, which exploits the $x-y$ coordinates of a mid-IR laser for imaging in combination with the electrospray ionization mechanism for a considerable molecular mass range coverage. We aimed to enhance the technique's sensitivity and/or selectivity by online chemical reactions following hardware improvements. We target rapid screening of molecular oxidation products, and improved structure elucidation approaches. Finally, apart from the established methods for biological samples, we alternatively intended to expand the scope and understanding of this technique for samples from material sciences. 


\section{Outline of this Thesis}

In order to broaden the scope of ambient laser ablation electrospray ionization (LAESI) mass spectrometry imaging, sample surface properties in the absence of endogenous water were investigated. Chapter 2 describes the analysis of several polymers containing amide or amine groups in the absence of water. For the analysis of finishes deposited on the polymer fibers, a thin layer of ice was accumulated on the fiber, as a laser absorbing matrix (IR-MALDESI). Imaging experiments were designed to detect synthetic fiber surface layer defects in a transient LAESI and IR-MALDESI mode.

In Chapter 3, reactive LAESI was introduced for sensitivity and selectivity enhancements. Hardware improvements in the form of a reaction tube were implemented to enable online chemical reactions. "Click chemistry" reactions were studied online in a time-resolved way by using different reaction tube lengths to vary the time difference between the laser pulse and the onset of the MS signal. The feasibility of reactive LAESI mass spectrometry imaging was shown with the analysis of an image of the WUR logo containing a frozen model compound solution.

The rapid screening of molecular oxidation products which are similar to phase I oxidation metabolism is shown in Chapter 4. Titanium dioxide $\left(\mathrm{TiO}_{2}\right)$ was used as photocatalyst material for oxidation reactions. Two approaches were developed, a "static" approach in which glass slides were coated with $\mathrm{TiO}_{2}$ and sample solvent droplets on these slides were irradiated with UV light before LAESI analysis. The $2^{\text {nd }}$ approach was an online time-resolved method exploiting a sample cup containing a $\mathrm{TiO}_{2}$ nanoparticle suspension, which could identify sequential oxidation steps.

A new structure elucidation approach for ambient mass spectrometry was introduced in Chapter 5. Hydrogen-deuterium exchange was used to obtain additional information on molecular structures. Exchangeable protons, e.g., in $-\mathrm{OH}$ and $-\mathrm{NH}$ moieties, can rapidly exchange for deuterons and increase the detected $\mathrm{m} / \mathrm{z}$ value, providing information on analyte functional groups. This approach can in addition to accurate mass determination and MS/MS workflows be used to elucidate the molecular structures of unknowns. The method was demonstrated with the analysis of endogenous arginine and oligosaccharides directly from an orange slice as well as with a ubiquitin spiked muscle tissue sample.

Chapter 6 contains the general discussion of the topics presented in this thesis and provides insights into the limitations, social impact and future perspectives of the research. 


\section{References}

1 Becker, L.; Bellow, S.; Carré, V., et al. Anal. Chem. 2017, 89, 7099-7106.

2 Weissleder, R.; Pittet, M. J. Nature 2008, 452, 580.

3 Weissleder, R.; Nahrendorf, M. Proc. Natl. Acad. Sci. U.S.A 2015, 112, 14424-14428.

4 Jones, T. Eur. J. Nucl. Med. 1996, 23, 807-813.

5 Imamura, T.; Saitou, T.; Kawakami, R. Cancer Sci. 2018, 109, 912-918.

6 Lauterbur, P. C. Nature 1973, 242, 190-191.

7 Shukla, A. K.; Kumar, U. J. Med. Phys. 2006, 31, 13-21.

8 Moses, W. W. Nucl. Instrum. Methods Phys. Res. A 2011, 648, S236-S240.

9 Lichtman, J. W.; Conchello, J.-A. Nat. Methods 2005, 2, 910.

10 Potter, K. J. Cell. Biochem. 2002, 87, 147-153.

11 Bloch, F.; Hansen, W. W.; Packard, M. Phys. Rev. 1946, 69, 127.

12 Purcell, E. M.; Torrey, H. C.; Pound, R. V. Phys. Rev. 1946, 69, 37-38.

13 Friebolin, H. In Basic one- and two-dimensional NMR spectroscopy, 5th edition, WileyVHC, 2010.

14 Perez, J. M.; Josephson, L.; O'Loughlin, T., et al. Nat. Biotechnol. 2002, 20, 816.

15 Hu, D.-H.; Sheng, Z.-H.; Zhang, P.-F., et al. Nanoscale 2013, 5, 1624-1628.

16 Chughtai, K.; Heeren, R. M. A. Chem. Rev. 2010, 110, 3237-3277.

17 Caprioli, R. M. Proteomics 2014, 14, 807-809.

18 McDonnell, L. A.; Angel, P. M.; Lou, S., et al. Adv. Cancer Res. 2017, 134, 283-290.

19 Dill, A. L.; Eberlin, L. S.; Ifa, D. R.; Cooks, R. G. Chem. Commun. 2011, 47, 27412746.

20 Prideaux, B.; Stoeckli, M. J. Proteom. 2012, 75, 4999-5013.

21 Takai, N.; Tanaka, Y. Bioanalysis 2015, 7, 2639-2648.

22 Tiddia, M.; Mihara, I.; Seah, M. P., et al. Appl. Mater. Interfaces 2019, 11, 4500-4506.

23 Endres, K. J.; Hill, J. A.; Lu, K., et al. Anal. Chem. 2018, 90, 13427-13433.

24 Doerr, A. Nat. Methods 2018, 15, 32.

25 Mattauch, J.; Herzog, R. Z. Phys. 1934, 89, 786-795.

26 Bristow, A. W. T.; Webb, K. S. J. Am. Soc. Mass Spectrom. 2003, 14, 1086-1098.

27 de Hoffmann, E.; Stroobant, V. In Mass Spectrometry: Principles and Applications, 3rd Edition, Wiley-Interscience, 2007.

28 Yost, R. A.; Enke, C. G. J. Am. Chem. Soc. 1978, 100, 2274-2275.

29 March, R. E. J. Mass Spectrom. 1997, 32, 351-369.

30 Marshall, A. G.; Hendrickson, C. L.; Jackson, G. S. Mass Spectrom. Rev. 1998, 17, 135.

$31 \mathrm{Hu}, \mathrm{Q}$.; Noll, R. J.; Li, H., et al. J. Mass Spectrom. 2005, 40, 430-443.

32 Stephens, W. E. Phys. Rev. 1946, 69, 674-674.

33 Wiley, W. C.; McLaren, I. H. Rev. Sci. Instrum. 1955, 26, 1150-1157.

34 Mcluckey, S. A.; Wells, J. M. Chem. Rev. 2001, 101, 571-606.

35 Allen, J. S. Phys. Rev. 1939, 55, 966-971.

36 Allen, J. S. Rev. Sci. Instrum. 1947, 18, 739-749.

37 Fenn, J.; Mann, M.; Meng, C., et al. Science 1989, 246, 64-71.

38 Karas, M.; Hillenkamp, F. Anal. Chem. 1988, 60, 2299-2301.

39 Kebarle, P.; Tang, L. Anal. Chem. 1993, 65, 972-986.

40 Tang, K.; Gomez, A. Phys. Fluids 1994, 6, 2317-2332.

41 Gomez, A.; Tang, K. Phys. Fluids 1994, 6, 404-414.

42 van Berkel, W. J. H.; van den Heuvel, R. H. H.; Versluis, C.; Heck, A. J. R. Protein Sci. 2000, 9, 435-439.

43 Snijder, J.; Rose, R. J.; Veesler, D., et al. Angew. Chem. Int. Ed. 2013, 52, 4020-4023.

44 Fenn, J. B.; Mann, M.; Meng, C. K., et al. Mass Spectrom. Rev. 1990, 9, 37-70.

45 Zenobi, R.; Knochenmuss, R. Mass Spectrom. Rev. 1998, 17, 337-366.

46 Nielen, M. W. F. Mass Spectrom. Rev. 1999, 18, 309-344. 
47 Karas, M.; Krüger, R. Chem. Rev. 2003, 103, 427-440.

48 van Kampen, J. J. A.; Burgers, P. C.; de Groot, R., et al. Mass Spectrom. Rev. 2011, 30, 101-120.

49 O'Rourke, M. B.; Djordjevic, S. P.; Padula, M. P. Mass Spectrom. Rev. 2018, 37, 217228.

50 McLafferty, F. W. Acc. Chem. Res. 1980, 13, 33-39.

51 Kind, T.; Tsugawa, H.; Cajka, T., et al. Mass Spectrom. Rev. 2018, 37, 513-532.

52 Kiss, A.; Smith, D. F.; Reschke, B. R., et al. Proteomics 2014, 14, 1283-1289.

53 Altuntaş, E.; Schubert, U. S. Anal. Chim. Acta 2014, 808, 56-69.

54 Luxembourg, S. L.; Mize, T. H.; McDonnell, L. A.; Heeren, R. M. A. Anal. Chem. 2004, 76, 5339-5344.

55 Todd, P. J.; Schaaff, T. G.; Chaurand, P.; Caprioli, R. M. J. Mass Spectrom. 2001, 36, 355-369.

56 Luxembourg, S. L.; Vaezaddeh, A. R.; Amstalden, E. R., et al. Rapid Commun. Mass Spectrom. 2006, 20, 3435-3442.

57 Froesch, M.; Luxembourg, S. L.; Verheijde, D.; Heeren, R. M. A. Eur. J. Mass Spectrom. 2010, 16, 35-45.

58 Kiss, A.; Smith, D. F.; Jungmann, J. H.; Heeren, R. M. A. Rapid Commun. Mass Spectrom. 2013, 27, 2745-2750.

59 Soltwisch, J.; Göritz, G.; Jungmann, J. H., et al. Anal. Chem. 2014, 86, 321-325.

60 Klerk, L. A.; Altelaar, A. F. M.; Froesch, M., et al. Int. J. Mass spectrom. 2009, 285, $19-25$.

61 MacAleese, L.; Stauber, J.; Heeren, R. M. A. Proteomics 2009, 9, 819-834.

62 Castaing, R.; Slodzian, G. J. Microscopie 1962, 1, 395-410.

63 Veryovkin, I. V.; Calaway, W. F.; Tripa, C. E.; Pellin, M. J. Nucl. Instrum. Methods Phys. Res. B 2007, 261, 508-511.

64 Kaletaş, B. K.; van der Wiel, I. M.; Stauber, J., et al. Proteomics 2009, 9, 2622-2633.

65 Benninghoven, A.; Hagenhoff, B.; Niehuis, E. Anal. Chem. 1993, 65, 630A-640A.

66 Diehnelt, C. W.; Van Stipdonk, M. J.; Schweikert, E. A. Int. J. Mass spectrom. 2001, 207, 111-122.

67 Chandra, S.; Morrison, G. H. Int. J. of Mass Spectrom. Ion Process. 1995, 143, 161176.

68 Strick, R.; Strissel, P. L.; Gavrilov, K.; Levi-Setti, R. J. Cell Biol. 2001, 155, 899-910.

69 Pacholski, M. L.; Cannon Jr., D. M.; Ewing, A. G.; Winograd, N. Rapid Commun. Mass Spectrom. 1998, 12, 1232-1235.

70 Magnusson, Y.; Friberg, P.; Sjövall, P., et al. Clin. Physiol. Funct. Imaging 2008, 28, 202-209.

71 Fernandez-Lima, F. A.; DeBord, J. D.; Schweikert, E. A., et al. Surf. Interface Anal. 2013, 45, 294-297.

72 Delcorte, A.; Garrison, B. J. J. Phys. Chem. B 2003, 107, 2297-2310.

73 Altelaar, A. F. M.; Klinkert, I.; Jalink, K., et al. Anal. Chem. 2006, 78, 734-742.

74 Winograd, N.; Garrison, B. J. Annu. Rev. Phys. Chem. 2010, 61, 305-322.

75 Heeren, R. M. A.; McDonnell, L. A.; Amstalden, E., et al. Appl. Surf. Sci. 2006, 252, 6827-6835.

76 Watrous, J. D.; Alexandrov, T.; Dorrestein, P. C. J. Mass Spectrom. 2011, 46, 209222.

77 Caprioli, R. M.; Farmer, T. B.; Gile, J. Anal. Chem. 1997, 69, 4751-4760.

78 Chaurand, P.; Schriver, K. E.; Caprioli, R. M. J. Mass Spectrom. 2007, 42, 476-489.

79 Bodzon-Kulakowska, A.; Suder, P. Mass Spectrom. Rev. 2016, 35, 147-169.

80 Dettmer, K.; Aronov, P. A.; Hammock, B. D. Mass Spectrom. Rev. 2007, 26, 51-78.

81 Wu, C.; Dill, A. L.; Eberlin, L. S., et al. Mass Spectrom. Rev. 2013, 32, 218-243.

82 Garden, R. W.; Sweedler, J. V. Anal. Chem. 2000, 72, 30-36.

83 Cillero-Pastor, B.; Heeren, R. M. A. J. Proteome Res. 2014, 13, 325-335. 
84 Luxembourg, S. L.; McDonnell, L. A.; Duursma, M. C., et al. Anal. Chem. 2003, 75, 2333-2341.

85 Cha, S.; Zhang, H.; Ilarslan, H. I., et al. Plant J. 2008, 55, 348-360.

86 Thomas, A.; Charbonneau, J. L.; Fournaise, E.; Chaurand, P. Anal. Chem. 2012, 84, 2048-2054.

87 Takáts, Z.; Wiseman, J. M.; Gologan, B.; Cooks, R. G. Science 2004, 306, 471-473.

88 Cody, R. B.; Laramée, J. A.; Durst, H. D. Anal. Chem. 2005, 77, 2297-2302.

89 McEwen, C. N.; McKay, R. G.; Larsen, B. S. Anal. Chem. 2005, 77, 7826-7831.

90 Takáts, Z.; Cotte-Rodriguez, I.; Talaty, N., et al. Chem. Commun. 2005, 1950-1952.

$91 \mathrm{Na}, \mathrm{N}$.; Zhao, M.; Zhang, S., et al. J. Am. Soc. Mass Spectrom. 2007, 18, 1859-1862.

92 Ratcliffe, L. V.; Rutten, F. J. M.; Barrett, D. A., et al. Anal. Chem. 2007, 79, 60946101.

93 Wiseman, J. M.; Ifa, D. R.; Song, Q.; Cooks, R. G. Angew. Chem. Int. Ed. 2006, 45, 7188-7192.

94 Haddad, R.; Sparrapan, R.; Eberlin, M. N. Rapid Commun. Mass Spectrom. 2006, 20, 2901-2905.

95 Shiea, J.; Yuan, C.-H.; Huang, M.-Z., et al. Anal. Chem. 2008, 80, 4845-4852.

96 Nemes, P.; Vertes, A. Anal. Chem. 2007, 79, 8098-8106.

97 Chen, H.; Yang, S.; Wortmann, A.; Zenobi, R. Angew. Chem. Int. Ed. 2007, 46, 75917594.

98 Venter, A.; Nefliu, M.; Cooks, R. G. TrAC, Trends Anal. Chem. 2008, 27, 284-290.

99 Takáts, Z.; Wiseman, J. M.; Cooks, R. G. J. Mass Spectrom. 2005, 40, 1261.

100 Venter, A.; Sojka, P. E.; Cooks, R. G. Anal. Chem. 2006, 78, 8549.

101 Costa, A. B.; Cooks, R. G. Chem. Phys. Lett. 2008, 464, 1-8.

102 Garza, K. Y.; Feider, C. L.; Klein, D. R., et al. Anal. Chem. 2018, 90, 7785-7789.

103 Kocurek, K. I.; Griffiths, R. L.; Cooper, H. J. J. Mass Spectrom. 2018, 53, 565-578.

104 Towers, M. W.; Karancsi, T.; Jones, E. A., et al. J. Am. Soc. Mass Spectrom. 2018, 29, 2456-2466.

105 Wu, C.; Ifa, D. R.; Manicke, N. E.; Cooks, R. G. Anal. Chem. 2009, 81, 7618-7624.

106 Barbara, J. E.; Eyler, J. R.; Powell, D. H. Rapid Commun. Mass Spectrom. 2008, 22, 4121-4128.

107 Song, Y.; Cooks, R. G. J. Mass Spectrom. 2007, 42, 1086-1092.

108 Chen, H.; Cotte-Rodriguez, I.; Cooks, R. G. Chem. Commun. 2006, 597-599.

109 Nefliu, M.; Cooks, R. G.; Moore, C. J. Am. Soc. Mass Spectrom. 2006, 17, 1091-1095.

110 Nemes, P.; Huang, H.; Vertes, A. Phys. Chem. Chem. Phys. 2012, 14, 2501-2507.

111 Nemes, P.; Barton, A. A.; Li, Y.; Vertes, A. Anal. Chem. 2008, 80, 4575-4582.

112 Vertes, A.; Nemes, P.; Shrestha, B., et al. Appl. Phys. A 2008, 93, 885-891.

113 Sripadi, P.; Nazarian, J.; Hathout, Y., et al. Metabolomics 2009, 5, 263-276.

114 Nemes, P.; Barton, A. A.; Vertes, A. Anal. Chem. 2009, 81, 6668-6675.

115 Nemes, P.; Vertes, A. Methods Mol. Biol. 2010, 656, 159-171.

116 Nielen, M. W. F.; van Beek, T. A. Anal. Bioanal. Chem. 2014, 406, 6805-6815.

117 Shrestha, B.; Patt, J. M.; Vertes, A. Anal. Chem. 2011, 83, 2947-2955.

118 Shrestha, B.; Vertes, A. J. Vis. Exp. 2010, e2144.

119 Shrestha, B.; Vertes, A. Anal. Chem. 2009, 81, 8265-8271.

120 Vaikkinen, A.; Shrestha, B.; Nazarian, J., et al. Anal. Chem. 2012, 85, 177-184. 


\section{Chapter 2}

\section{Ambient Characterization of Synthetic Fibers by Laser Ablation Electrospray Ionization Mass Spectrometry}

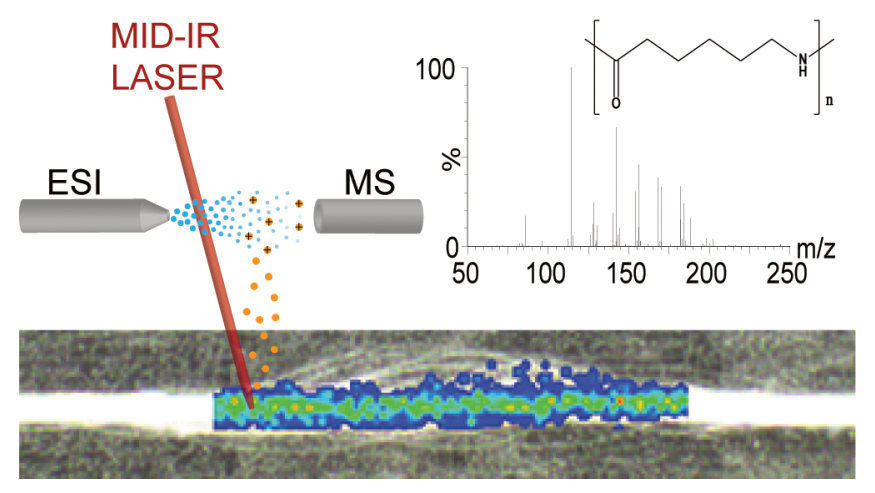

This Chapter was published as:

Fred A. M. G. van Geenen, Maurice C. R. Franssen, Anton H. M. Schotman, Han Zuilhof and Michel W. F. Nielen. Ambient Characterization of Synthetic Fibers by Laser Ablation Electrospray lonization Mass Spectrometry. Analytical Chemistry, 2017, 89, 4031-4037. https://pubs.acs.org/doi/abs/10.1021/acs.analchem.6b04641

Note: permissions related to the material in this chapter should be directed to the American Chemical Society. 


\section{Abstract}

Direct analysis of synthetic fibers under ambient conditions is highly desired to identify the polymer, the finishes applied and irregularities that may compromise its performance and value. In this paper, laser ablation electrospray ionization ion mobility timeof-flight mass spectrometry (LAESI-IMS-TOF-MS) was used for the analysis of synthetic polymers and fibers. The key to this analysis was the absorption of laser light by aliphatic and aromatic nitrogen functionalities in the polymers. Analysis of polyamide (PA) 6, 46, 66, and 12 pellets and PA 6, 66, polyaramid and M5 fibers yielded characteristic fragment ions without any sample pretreatment, enabling their unambiguous identification. Synthetic fibers are, in addition, commonly covered with a surface layer for improved adhesion and processing. The same setup, but operated in a transient infrared matrix-assisted laser desorption electrospray ionization (IR-MALDESI) mode, allowed the detailed characterization of the fiber finish layer and the underlying polymer. Differences in finish layer distribution may cause variations in local properties of synthetic fibers. Here we also show the feasibility of mass spectrometry imaging (MSI) of the distribution of a finish layer on the synthetic fiber and the successful detection of local surface defects. 


\section{Introduction}

Synthetic fibers such as polyamide and polyester are widely used in many industrial materials, fabrics, clothes, etc., and their importance can hardly be overemphasized. Highperformance fibers such as polyaramid are heat resistant and stronger than steel on an equal weight basis. Because of these properties, they can be used in, for example, ballistic vests, cables, optical fiber reinforcement, as well as in rubber reinforcement such as in tires, highpressure hoses, conveyer belts, etc. The properties of synthetic fibers and their interaction with surrounding materials are strongly influenced by the chemical treatment of their surface. ${ }^{1-}$ ${ }^{4} \mathrm{~A}$ large number of different types of surface layers can be used to adapt the fiber properties to the desired applications. Surface layers are used for processing, e.g., to reduce friction, electrostatic charging, and abrasion ${ }^{5}$ or to allow better adhesion to other polymers in blends. ${ }^{6-}$ ${ }^{8}$ Differences in the amount and distribution of the surface layer lead to variations in properties of the fiber. ${ }^{9}$ Such surface defects might result in weak spots leading to abrasion or localized reduced adhesion, ${ }^{10}$ which could be detrimental in high-performance applications.

Mass spectrometry (MS) is an excellent tool for the characterization of polymers as well as polymer surfaces and additives. The use of MS in polymer analysis has been reviewed recently. ${ }^{11,12}$ Secondary ion mass spectrometry (SIMS) is commonly used for the analysis of polymers and synthetic fibers. ${ }^{13-26}$ SIMS typically produces small fragment ions for solid polymer samples, e.g., CN- in PA fibers. ${ }^{18,20,21}$ Oligomers of PA of 6 up to 24 repeating units were observed after dissolution of the fiber in trifluoroacetic acid, deposition on a silver substrate, and SIMS analysis. ${ }^{13}$ Also, matrix-assisted laser desorption ionization MS (MALDI$M S$ ) is frequently used for the characterization of polymers. ${ }^{27-33}$ Applications range from mass spectrometry imaging of polymer membranes, showing polymer distributions and contaminations on membrane surfaces, ${ }^{32}$ to the characterization of branching in polyaramid fibers. ${ }^{33}$ The analysis of fibers by SIMS or MALDI-MS is, however, hampered by sample pretreatment requirements and ionization under vacuum conditions. Analysis under ambient conditions without any sample pretreatment would be very useful for synthetic fibers in order to quickly identify the material, finish layers, and defects without introducing any pretreatmentinduced bias.

Direct analysis in real time (DART) is an ambient MS technique first introduced in 2005 by Cody. ${ }^{34}$ DART has been used for the detection of additives in different polymer materials such as softeners and stabilizers or degradation products, ${ }^{35-40}$ but the polymer material itself was not detected since DART relies on thermal desorption. Some progress has been made to fingerprint insoluble polymers under ambient conditions using thermal-assisted atmospheric pressure glow discharge (TA-APGD) following fixation of the sample on a heated stage. ${ }^{41}$ Other plasma-based techniques such as plasma-assisted desorption ionization (PADI) and flowing afterglow atmospheric pressure glow discharge (FA-APGD) were reported to successfully identify different insoluble polymers. ${ }^{42,43}$ While certainly useful, the main 
drawbacks of these techniques relate to long stabilization times and relatively low spatial resolution that precludes mass spectrometry imaging (MSI) of fibers. Another ambient ionization MS technique used for identification of polymer species is desorption electrospray ionization (DESI). ${ }^{44-47} \mathrm{DESI}$ relies on the solubility of sample material for desorption and subsequent ESI-like ionization mechanisms. ${ }^{48}$ For the analysis of hydrophobic polymers, such as poly(methyl methacrylate) and polymethylstyrene, methanol solutions containing formic acid or salts such as lithium bromide or silver nitrate were used. ${ }^{49}$ Without water in the solution, however, DESI is less able to create higher charge states which limits polymer analysis applications.

Alternatively, laser-based techniques are fast and offer much smaller spot sizes. Electrospray-assisted laser desorption ionization (ELDI) was used to characterize dried polymer standard solutions of PPG 1000, PMMA 1300, and PEG 1500 on the surface of a steel sample plate. ${ }^{50}$ Tuning of the laser wavelength to specific polymer absorption bands removed the necessity of an external matrix and allowed successful ablation of bulk polymer material, as demonstrated with a free electron laser operated at a wavelength of $3.43 \mu \mathrm{m}$ to interact with the aliphatic $\mathrm{CH}$ stretch vibration of polystyrene. ${ }^{51}$ Ablated material can subsequently be postionized by different techniques such as electrospray ionization, chemical ionization or photoionization. ${ }^{52-54}$

Laser ablation electrospray ionization (LAESI) is an ambient (imaging) MS technique first introduced by Nemes and Vertes in $2007 .{ }^{52}$ It uses a mid-infrared laser producing a wavelength of $2.94 \mu \mathrm{m}$ that addresses hydroxyl functionalities, such as endogenous water molecules in biomaterials. In principle, the same wavelength can also be absorbed by aliphatic or aromatic nitrogen functionalities in polymers. The absorbed energy could break the polymer chains and ablate characteristic fragments under ambient conditions for subsequent mass spectrometric analysis. In the absence of laser-absorbing functional groups, a small amount of water vapor can be condensed on a sample surface to absorb the laser energy, as was shown for the detailed structure elucidation of dyes on fabrics by infrared matrix-assisted laser desorption electrospray ionization (IR-MALDESI). ${ }^{55}$ Detailed characterization along the length of a $1 \mathrm{~mm}$ wide synthetic fiber was feasible. IR-MALDESI-MSI of dyes on textile fibers within a forensic context even showed single filament images as small as $10 \mu \mathrm{m}$ in diameter ${ }^{56}$ When combining $\mathrm{MSI}$ with ion mobility separation (IMS), an additional separation dimension becomes available to identify different polymer distributions, and to separate ions with the same mass to charge $(\mathrm{m} / \mathrm{z})$ ratio but having different collisional cross sections. ${ }^{57}$

In this research, we demonstrate the detailed MS characterization of different synthetic fibers such as PA $6,46,66,12$, polyaramid, and M5 by direct LAESI-MS. In addition, we provide full finish characterization, show the feasibility of mass spectrometry imaging of the fiber, the distribution of the finish layer, and the detection of local surface defects by LAESIIMS-TOF-MSI operated in a transient (ice)matrix-assisted laser ablation mode. 


\section{Experimental section}

\section{Materials}

Ultrapure water $\left(\mathrm{H}_{2} \mathrm{O}\right) 18.2 \mathrm{M} \Omega \times \mathrm{cm}$ at $25^{\circ} \mathrm{C}$ was freshly produced daily with a Millipore (Molsheim, France) Integral 3 system. Methanol (MeOH) LC-MS grade was purchased from VWR (Leuven, Belgium). Formic acid (FA) LC-MS grade was bought from Fisher Scientific (Geel, Belgium). Leucine-enkephalin (leu-enk) for lock mass-corrected mass calibration was purchased from Waters (Manchester, U.K.). Para-aramid trimer- $\mathrm{NH}_{2}$ (structure is show in Figure S1) and Twaron para-aramid yarn were provided by Teijin Aramid (Arnhem, The Netherlands). One standard yarn type (1680 dtex, f1000) was produced without applying a basic spin finish. In a second step, the "naked" yarn was treated with a finish, consisting of an aqueous solution (4.25\%) of Lansurf OA10 (polyethylene glycol (PEG) 400 monooleate, structure is shown in Figure S2), from Lankem (Dukinfield, United Kingdom). After application of this finish using a slit applicator, the yarn was dried by passing through a hot air oven (3.6 $\mathrm{s}$ at $180{ }^{\circ} \mathrm{C}$ ). The estimated (final) finish amount was $0.5 \%$ Lansurf OA10 (finish weight on yarn weight). Poly[2,6-diimidazo (4,5- $\left.\beta-4^{\prime}, 5^{\prime}-\varepsilon\right)$ pyridinylene-1,4-(2,5-dihydroxy)phenylene] (M5) fiber, polyethylene terephthalate (PET) fiber, and the PA fibers PA 6 ( $\mathrm{M}_{w}$ unknown), PA $66\left(\mathrm{M}_{\mathrm{w}} 27 \times 10^{3}\right), \mathrm{PA} 66\left(\mathrm{M}_{\mathrm{w}} 46 \times 10^{3}\right)$, and PA $66\left(\mathrm{M}_{\mathrm{w}} 33 \times 10^{3}\right)$ were from laboratory stock, as were poly(methyl methacrylate) (PMMA) ( $\mathrm{M}_{w}$ unknown) pellets and the PA pellets PA 6 $\left(\mathrm{M}_{\mathrm{w}} 30 \times 10^{3}\right)$, PA $6\left(\mathrm{M}_{\mathrm{w}} 31 \times 10^{3}\right)$, PA $6\left(\mathrm{M}_{\mathrm{w}} 15 \times 10^{3}\right)$, PA $12\left(\mathrm{M}_{\mathrm{w}}\right.$ unknown), PA $66\left(\mathrm{M}_{\mathrm{w}} 22\right.$ $\left.\times 10^{3}\right)$, and PA $46\left(\mathrm{M}_{w}\right.$ unknown). Chemical structures of fibers and pellets are given in Figure 1.<smiles>CNCCCCCC(C)=O</smiles><smiles>[B]C(C)(C)CCCCCCCCCCCNC(C)(C)C</smiles><smiles>CNCCCCNC(=O)CCCCC(C)C</smiles><smiles>CC(C)(C)CCCCCCNC(=O)CCCCCCNCCC(=O)O</smiles>
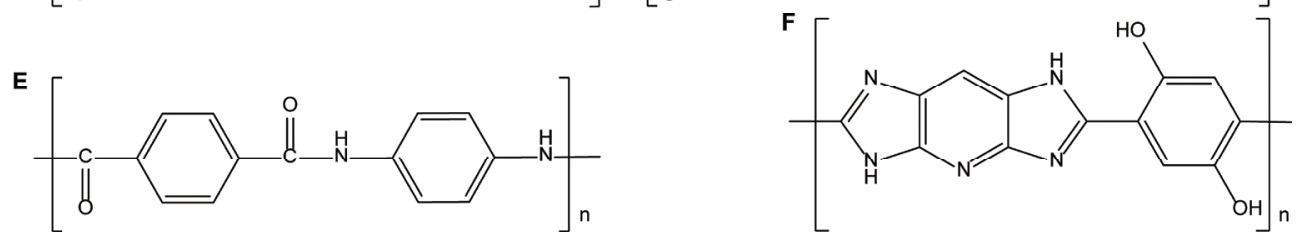

Figure 1. Chemical structures of (A) PA 6, (B) PA 12, (C) PA 46, (D) PA 66, (E) polyaramid, and (F) M5. 


\section{LAESI-MS of Polymers and Fibers}

A Protea Biosciences (Morgantown, WV) LAESI DP-1000 system was coupled to a Waters (Manchester, U.K.) Synapt G2S traveling wave ion mobility time-of-flight mass spectrometer and used for the analysis of all sample materials. Both polymer pellets and synthetic fibers were directly mounted with Tesa double-sided tape (Hamburg, Germany) to the sample stage that was kept at $10{ }^{\circ} \mathrm{C}$. LAESI desktop software v.2.0.1.3 (Protea Biosciences) was used to control experimental parameters of the LAESI system. The Nd:YAG optical parametric oscillator mid infrared laser $(2.94 \mu \mathrm{m})$ was set to $100 \%$ laser power $(\Phi 3.2$ $\mathrm{J} / \mathrm{cm}^{2}$ ) and 10 pulses with a specified pulse length of $5 \mathrm{~ns}$ were acquired on every spot $(\varnothing 200$ $\mu \mathrm{m})$ at a frequency of $10 \mathrm{~Hz}$ with a between spot interval of $200 \mu \mathrm{m}$. A solution of $\mathrm{MeOH}-\mathrm{H}_{2} \mathrm{O}$ (1:1) with $0.1 \% \mathrm{FA}$ and $40 \mathrm{ng} / \mathrm{mL}$ leu-enk was used as electrospray solvent at a flow rate of 1 $\mu \mathrm{L} / \mathrm{min}$. Electrospray voltage was set at $\sim 3.5 \mathrm{kV}$ in order to have a stable Taylor cone. Nitrogen was used as LAESI bath gas at $20 \mathrm{~L} / \mathrm{h}$. The Synapt G2S was controlled by Masslynx v4.1 SCN 883 (Waters) and operated in positive ion TOF-MS resolution mode, $\mathrm{m} / \mathrm{z}$ range 50 $1200 \mathrm{Da}$, scan time $1 \mathrm{~s}$, and source and interface temperatures were both set at $150{ }^{\circ} \mathrm{C}$. For ion mobility TOF-MS the IMS wave velocity was set to $650 \mathrm{~m} / \mathrm{s}$ and the transfer velocity at $1200 \mathrm{~m} / \mathrm{s}$. Background-subtracted mass spectra were created using the "combine spectrum" function in Masslynx: five scans, each corresponding to 10 laser pulses were averaged, and 50 scans of the electrospray background were subtracted. Proteaplot v2.0.8.5 (Protea Biosciences) was used to create maximum intensity ion maps. Driftscope v2.7 (Waters) was used to select the different polymer distributions within the $\mathrm{m} / \mathrm{z}$ versus drift time space and to clean the spectral background.

\section{IR-MALDESI and LAESI Mass Spectrometry Imaging of Fibers with a Finish Layer}

For MSI of the finish layers on the fibers, the same instrument and experimental conditions were used as described in the previous section, with the exception of the sample stage temperature which was set to $-19^{\circ} \mathrm{C}$, starting $15 \mathrm{~min}$ before analysis in order to achieve a thin layer of ice on the sample. Consequently, instead of LAESI, ice-assisted LAESI or IRMALDESI ${ }^{55,56}$ occurred for typically the first two or three (out of ten) laser pulses applied. Optical images of the $10 \mathrm{~cm} \times 7 \mathrm{~cm}$ sample stage were obtained and used to superimpose the ion maps. A $60 \times 6$ pattern (350 sample locations) with an interval of $400 \mu \mathrm{m}$ was acquired around the synthetic fiber area on the optical image. The MSI analysis, including the recording of electrospray background, had a total runtime of $45 \mathrm{~min}$. 


\section{Results and Discussion}

\section{LAESI Fingerprinting of Synthetic Polymers}

LAESI experiments conventionally use a mid-infrared laser $(2.94 \mu \mathrm{m})$ to efficiently transfer laser energy to the strong $\mathrm{OH}$ stretch vibration of water-containing (mostly biological) samples in order to ablate sample material prior to ionization by electrospray for subsequent $\mathrm{MS}$ analysis. Besides the strong $\mathrm{OH}$ absorption band, also $\mathrm{NH}$ stretch vibrations of amines and amides absorb at this wavelength. So initially, we examined LAESI-TOF-MS as a tool for rapid ambient identification of solid $\mathrm{NH}$-containing polymer materials without any sample pretreatment. Figure 2 presents the background-subtracted mass spectra of different polyamides. The mass spectra obtained show characteristic ions and confirm the capability of LAESI fingerprinting of polyamides. The mass accuracies corresponding to the proposed elemental compositions are provided in Table S1. The mass spectrum of PA 6 (Figure 2A) shows a high abundance ion at $m / z 114.091$ having the elemental composition $\left[\mathrm{C}_{6} \mathrm{H}_{12} \mathrm{NO}\right]^{+}$, which represents residual caprolactam monomer and ions formed by cleavage of the amide bond. This high abundance ion differentiates PA 6 from the other polyamides and was also observed by Klun after acid hydrolysis of PA 6 and subsequent electrospray MS analysis. ${ }^{58}$ In LAESI analysis, although at lower intensity, $m / z$ values $128.105\left[\mathrm{C}_{7} \mathrm{H}_{14} \mathrm{NO}^{+}, 142.121\right.$ $\left[\mathrm{C}_{8} \mathrm{H}_{16} \mathrm{NO}\right]^{+}, 156.138\left[\mathrm{C}_{9} \mathrm{H}_{18} \mathrm{NO}\right]^{+}, 170.153\left[\mathrm{C}_{10} \mathrm{H}_{20} \mathrm{NO}\right]^{+}$, and $184.168\left[\mathrm{C}_{11} \mathrm{H}_{22} \mathrm{NO}\right]^{+}$were additionally detected. Two extra PA 6 samples were analyzed providing similar results, the background-subtracted mass spectra are presented in Figures S3 and S4. The LAESI mass spectrum of PA 12 (Figure 2B) shows a single ion at $\mathrm{m} / \mathrm{z} 198.188$ having the elemental composition of $\left[\mathrm{C}_{12} \mathrm{H}_{24} \mathrm{NO}\right]^{+}$. Like PA 6, this ion represents residual monomer and/or cleavage of the amide bond yielding the ion for the repeating unit of PA 12. In contrast to PA 6 and 12, PA 46 and PA 66 are synthesized from two different monomers; therefore their mass spectra will be more complex. Cleavage of the amide bond will lead to fragments containing at least either two nitrogen atoms or two oxygen atoms, and furthermore cleavage of carbon-carbon bonds could yield fragments that contain the intact amide bond. The LAESI mass spectrum of PA 46 is presented in Figure $2 \mathrm{C}$ and shows ions at $m / z$ values $128.105\left[\mathrm{C}_{7} \mathrm{H}_{14} \mathrm{NO}^{+}, 142.126\right.$ $\left[\mathrm{C}_{8} \mathrm{H}_{16} \mathrm{NO}\right]^{+}$, and $156.138\left[\mathrm{C}_{9} \mathrm{H}_{18} \mathrm{NO}\right]^{+}$that are consistent with the intact amide bond and carbon-carbon bond cleavages at different positions of the polymer backbone. In addition, the ion at $\mathrm{m} / \mathrm{z} 115.089\left[\mathrm{C}_{5} \mathrm{H}_{11} \mathrm{~N}_{2} \mathrm{O}\right]^{+}$is formed, which is characteristic for polyamides containing 1,4-butanediamine, like PA 46. This fragment contains the amide group, the four carbon atoms, and the final nitrogen yielding an elemental composition unique for PA 46. Finally, the mass spectrum of PA 66 is depicted in Figure 2D. Like PA 46 this spectrum contains ions at $m / z$ values $128.105\left[\mathrm{C}_{7} \mathrm{H}_{14} \mathrm{NO}\right]^{+}, 142.121\left[\mathrm{C}_{8} \mathrm{H}_{16} \mathrm{NO}\right]^{+}$, and $156.138\left[\mathrm{C}_{9} \mathrm{H}_{18} \mathrm{NO}\right]^{+}$. However, in PA 66 this ion series continues with the $\mathrm{m} / z$ values $170.153\left[\mathrm{C}_{10} \mathrm{H}_{20} \mathrm{NO}\right]^{+}$and 184.168 $\left[\mathrm{C}_{11} \mathrm{H}_{22} \mathrm{NO}\right]^{+}$that could not be found in PA 46. In addition, the absence of $\mathrm{m} / \mathrm{z}$ values 115.089 (PA 46), 198.188 (PA 12), and high intensity $m / z 114.091$ (PA 6) makes this spectrum unique 
for identification of PA 66 polymer. It should be noted that MS/MS data acquisition following precursor ion selection of specific LAESI induced fragment ions may add additional evidence for the identity of the polymers but was not further investigated here.

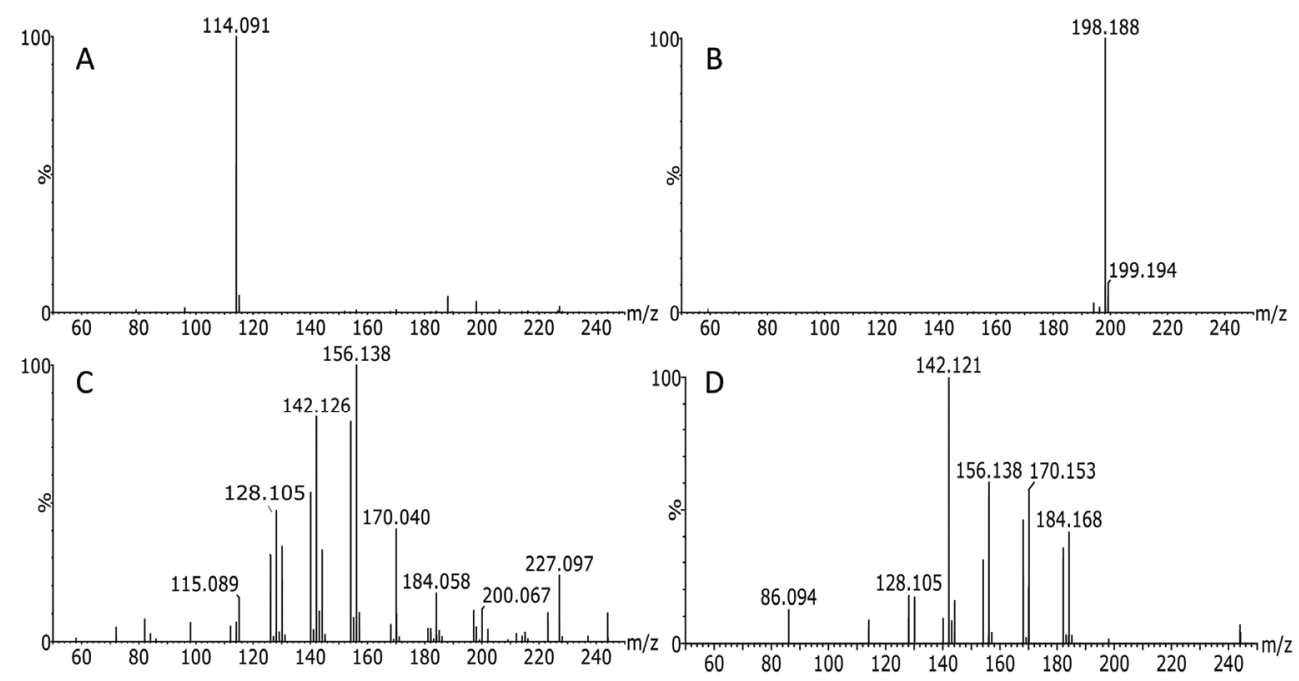

Figure 2. LAESI-TOF-MS background-subtracted mass spectra of polyamide pellets (A) PA 6, (B) PA 12, (C) PA 46, and (D) PA 66.

In the MS analysis of polyamides with other ionization techniques like SIMS, MALDI and sequential pyrolysis field desorption, PA distributions of multiple intact oligomers have been detected. ${ }^{13,59-61}$ Differentiation between, e.g., PA 6 and PA 66 could not be achieved. With FA-APGD, the cyclic monomer ion of PA 66 at $\mathrm{m} / \mathrm{z} 227$ could be detected. ${ }^{43}$ In LAESI, we detect characteristic polymer fragments in the low mass range. This is in contrast with the suggestion that LAESI is a low energy ionization technique comparable to ESI. ${ }^{22}$ Note that in the present situation the $\mathrm{NH}$ stretching vibration within the amide bond is addressed in the absence of water and, as a result, the laser energy is not dissipated by an excess of hydroxyl moieties from the sample matrix.

\section{LAESI Fingerprinting of Synthetic Fibers}

Similarly, LAESI-MS can be used to identify the polymer used in synthetic fibers. Different $\mathrm{NH}$-containing fibers, such as the polyamides PA 6 and PA 66 and the aromatic fibers polyaramid and M5, were analyzed by LAESI-TOF-MS. Additionally, the obtained data were used to generate spatial ion maps superimposed onto the camera images of the fibers in order to explore LAESI-MSI possibilities. Figure 3 shows ion maps and backgroundsubtracted mass spectra of (A) PA 6, (B) PA 66, (C) polyaramid, and (D) M5 fibers. The LAESI mass spectrum of PA 6 (Figure $3 A$ ) shows the same fragment ion at $m / z 114.091$ as obtained 
with the pellets, and a range of ions formed by breaking of carbon-carbon bonds in the polymer backbone. Most likely, PA 6 fiber contains less residual caprolactam monomer than the PA 6 polymer sample analyzed in Figure 2A. The additional ions in Figure 3A were also observed (but at much lower relative intensity) in the LAESI mass spectrum of the PA 6 polymer sample shown in Figure 2A. The LAESI mass spectra obtained from PA 66 fibers (Figure 3B and Figures S5 and S6) show similar fragment ions as seen for the PA 66 pellets in Figure 2D, strengthening our claim on the identification of PA polymers, regardless of processing into fibers or as raw polymer materials. The LAESI mass spectrum of the aromatic polyaramid fiber shows characteristic fragment ions at $\mathrm{m} / \mathrm{z} 107.059$ and $\mathrm{m} / \mathrm{z} 135.057$, which belong to the elemental compositions $\left[\mathrm{C}_{6} \mathrm{H}_{7} \mathrm{~N}_{2}\right]^{+}$and $\left[\mathrm{C}_{7} \mathrm{H}_{7} \mathrm{~N}_{2} \mathrm{O}\right]^{+}$, respectively. These fragments originate from the aromatic ring containing the two (1,4-) amine groups $\left[\mathrm{C}_{6} \mathrm{H}_{7} \mathrm{~N}_{2}\right]^{+}$and one additional carbonyl group from the amide $\left[\mathrm{C}_{7} \mathrm{H}_{7} \mathrm{~N}_{2} \mathrm{O}\right]^{+}$. The second aromatic fiber analyzed, M5, does not contain an amide bond (cf. Figure 1); nevertheless, the laser energy could be absorbed by either or both the secondary amine and hydroxyl groups. The background-subtracted LAESI mass spectrum of M5 given in Figure 3D shows fragment ions at $\mathrm{m} / \mathrm{z} 120.058, \mathrm{~m} / \mathrm{z}$ 143.059, and $m / z$ 144.053. The elemental compositions of these fragments are $\left[\mathrm{C}_{6} \mathrm{H}_{6} \mathrm{~N}_{3}\right]^{+}$, $\left[\mathrm{C}_{9} \mathrm{H}_{7} \mathrm{~N}_{2}\right]^{+}$, and $\left[\mathrm{C}_{8} \mathrm{H}_{6} \mathrm{~N}_{3}\right]^{+}$, respectively. Although these elemental compositions are difficult to explain and require multiple bond cleavages, these ions are unique for $\mathrm{M} 5$ among the $\mathrm{NH}$ containing fibers such as polyamides and polyaramides. Therefore, these ions provide the ability to quickly identify this fiber under ambient conditions. In comparison with, and in contrast to other ionization techniques like pyrolysis MS, ${ }^{62}$ MALDI and IR-MALDESI, 33,55,56 LAESI exclusively provides fragments in the low mass range. In SIMS, oxygen containing fragment ions from PA 6 filaments with $\mathrm{m} / \mathrm{z}$ values 31, 45, 114, and 227 were detected in positive ion mode by Yip et al. ${ }^{20}$ In comparison, the fragment ions $m / z 31$ and 45 were outside the mass range of our mass spectrometer, but $m / z$ value 114 corresponds to the mass found in this study for the repeating unit fragment of PA 6.

In addition to straightforward polymer identification, the feasibility of LAESI-MSI was explored in the same experiments. Reconstructed ion maps, superimposed on the optical images, show the distribution of the most abundant fragment ion in Figure 3A-D. In all cases the ion follows nicely the $1 \mathrm{~mm}$ wide fiber pattern on the sample stage. At $200 \mu \mathrm{m}$ spatial resolution, approximately five locations were analyzed over the fiber diameter. These results are very encouraging for imaging of finish layers on fibers. 

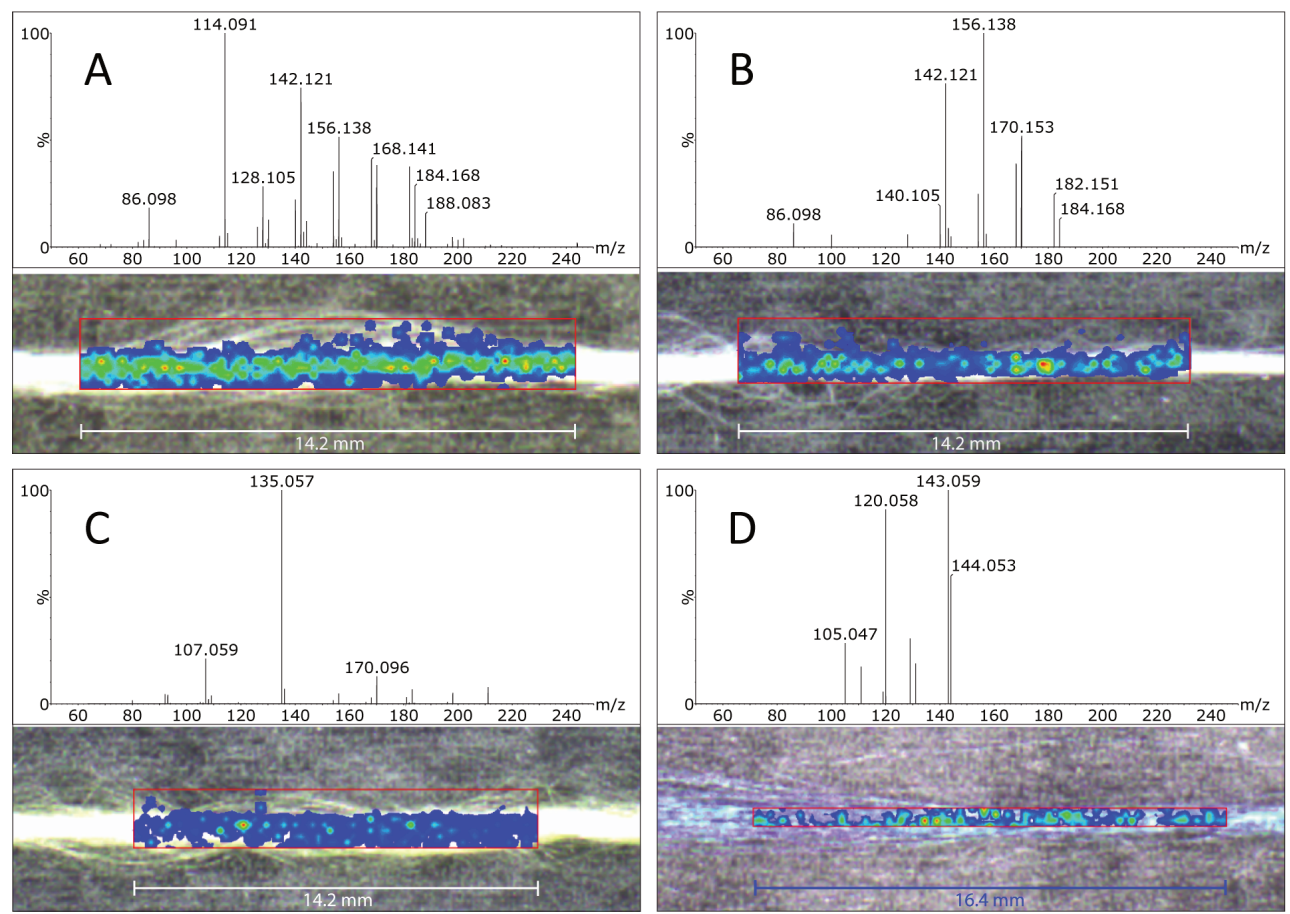

Figure 3. LAESI background-subtracted mass spectra and ion maps of (A) PA 6 fiber, (B) PA 66 fiber, (C) polyaramid fiber, and (D) M5 fiber. Ion maps show the highest intensity ion: (A) $m / z 114.091$, (B) $m / z 156.138$, (C) $m / z$ 135.057, and (D) $m / z 143.059$.

\section{LAESI Induced Fragmentation of Polymers}

In order to support our hypothesis that laser-induced fragmentation, initiated by absorption at $\mathrm{NH}$ functionalities, is the primary cause of the observed polymer characteristic fragment ions, we performed a range of complementary experiments. First, we extracted polyaramid fiber with $\mathrm{MeOH}$ (as described in the Supporting Information) to find out whether unreacted monomers, dimer, trimer, etc. could be present and contribute to the observed characteristic ions. The obtained ESI-MS background subtracted mass spectrum is depicted in Figure S7. Evidently, the finish is detected, but the fiber polymer fragments at $m / z 107.059$ and 135.057 (or dimers/trimers) were not.

To investigate the possibility of acid hydrolysis due to the close proximity of the formic acid-containing electrospray plume, we placed $10 \mathrm{~cm}$ of polyaramid fiber into $4 \mathrm{~mL}$ of electrospray solution for $30 \mathrm{~min}$. Subsequently, we analyzed this solution by ESI-MS and, as expected, did not detect any of the characteristic polymer fragments (data not shown). It should be kept in mind however that the $\mathrm{pH}$ experienced in electrospray droplets may be lower (due to charging and concentration effects of the spray) than that of the bulk spray solution. 
In addition to the analysis of polyaramid material with LAESI in the absence of water, aramid trimer- $\mathrm{NH}_{2}$ was also investigated. Figure S8A presents the ESI background subtracted mass spectrum of a $1 \mu \mathrm{g} / \mathrm{mL}$ trimer- $\mathrm{NH}_{2}$ solution in $\mathrm{MeOH}-\mathrm{H}_{2} \mathrm{O}(1: 1)+0.1 \% \mathrm{FA}$, showing ions at $m / z 347.149[\mathrm{M}+\mathrm{H}]^{+}, 369.126[\mathrm{M}+\mathrm{Na}]^{+}$, and $174.080[\mathrm{M}+2 \mathrm{H}]^{2+}$. Also, the LAESI background subtracted mass spectrum of a $10 \mu \mathrm{g} / \mathrm{mL}$ trimer- $\mathrm{NH}_{2}$ solution in $\mathrm{MeOH}-\mathrm{H}_{2} \mathrm{O}(1: 1)$ is depicted (Figure S8B), showing a single ion at $\mathrm{m} / \mathrm{z} 347.149[\mathrm{M}+\mathrm{H}]^{+}$. In contrast, LAESI analysis of trimer- $\mathrm{NH}_{2}$ powder (mass spectrum is shown in Figure $\mathrm{S} 8 \mathrm{C}$ ) did not display any of these masses, just the fragment ions $\mathrm{m} / \mathrm{z} 107.059$ and 135.057, as with the LAESI analysis of polyaramid fiber. This demonstrates that the observed fragmentation of the solid polymer pellets and fibers is a result of the dissipation of the laser energy. To examine the importance of absorption by $\mathrm{NH}$ functionalities within the polymer material, two materials without the $\mathrm{NH}$ functionality were measured: PMMA pellets and PET fiber. In accordance with our hypothesis, no ions from these polymers were detected (data not shown).

To further explore the effect of laser energy on the fragmentation of the polymer materials, PA 66 pellets were analyzed at different amounts of laser energy: 20, 40, 60, 80, and $100 \%$. Only at $100 \%$ laser power fragment ions, e.g., $\mathrm{m} / \mathrm{z} 142.121$ and 156.138 were detected. Most likely, this is a result of the laser ablation threshold, as is reported for IR laser ablation of other polymeric materials in the literature. ${ }^{51,63}$ Kappes et al. proposed an ablation mechanism well below decomposition temperature that is photomechanical, i.e., a stress due to the thermal expansion of the polymer. ${ }^{63}$ Here we hypothesize that in case the laser energy cannot be dissipated by the solvent (or endogenous water), the $\mathrm{NH}$-functionalities in the solid material will absorb the energy. As the stretch vibrations are unable to dissipate the excess of energy, multiple bond cleavages in the polymer backbone will occur, resulting in ablated polymer fragments.

\section{IR-MALDESI and LAESI MSI of Polyaramid Fiber with Finish}

A PEG 400 monooleate finish was applied to polyaramid fiber at a fiber treatment line as described in the Experimental Section. Investigations of the fiber containing this finish layer were conducted with transient IR-MALDESI and LAESI IMS-TOF-MSI in a single setup. The background-subtracted mass spectrum of polyaramid fiber containing $0.5 \%(\mathrm{~m} / \mathrm{m})$ finish is presented in Figure S9A and shows several polymer distributions. The characterization of this finish was supported by extracting the different polymer distributions from the $m / z$ versus ion mobility map provided in Figure 4. Three main distributions were assigned: (1) a $\left[(\mathrm{PEG})_{n}+\mathrm{NH}_{4}\right]^{+}$distribution (Figure S9B), (2) a $\left[(\mathrm{PEG})_{n} \text { monooleate }+\mathrm{NH}_{4}\right]^{+}$distribution (Figure $\mathrm{S} 9 \mathrm{C})$, and (3) a [(PEG) n dioleate $\left.+\mathrm{NH}_{4}\right]^{+}$distribution (Figure S9D). In addition to these polymer distributions, $\mathrm{m} / \mathrm{z} 283.264$ and $\mathrm{m} / \mathrm{z} 309.282$ are present, which are tentatively assigned to protonated oleic acid and an elimination product of the PEG ester of oleic acid (structure is shown in Figure S10), respectively. These results directly obtained from a fiber surface are, apart from the different cations and lower intensities, in excellent agreement with the LAESI 
data obtained from a $1 \%$ solution of the finish in $\mathrm{MeOH}$ (Figure $\mathrm{S} 11 \mathrm{~A}-\mathrm{F}$ ). In the latter, $[\mathrm{M}+\mathrm{Na}]^{+}$ ions dominated over $\left[\mathrm{M}+\mathrm{NH}_{4}\right]^{+}$ions. In other words, the transient ice-assisted LAESI provided the same low-energy ablation of the finish polymers from the surface as from solution. Similar cationized ethylene glycol oligomers were also found in the analysis of PEG 3000 with DESI;45 however, in our study no multiple charged polymers were observed due to a lower degree of PEG polymerization.

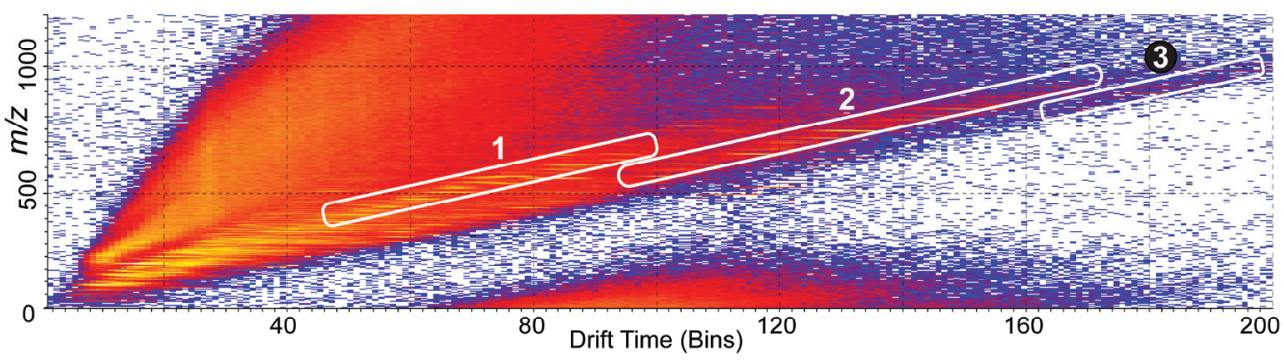

Figure 4. IR-MALDESI-IMS $\mathrm{m} / \mathrm{z}$ versus ion mobility map of $0.5 \%(\mathrm{~m} / \mathrm{m})$ PEG 400 monooleate applied as described in the Experimental Section on polyaramid fiber. Mass spectra of selected areas are shown for 1 in Figure S9B, for 2 in Figure S9C and for 3 in Figure S9D.

The distribution of the finish layer along the fiber and thus any surface defects, i.e., the areas without finish, could be visualized by reconstructing ion maps of the finish ions. An artificial defect of approximately $5 \mathrm{~mm}$ along the fiber length was produced by local heating of the fiber with $0.5 \%$ finish for $3 \mathrm{~s}$ with a soldering iron. Characteristic ions from both the fiber and the finish ion, e.g., $\left[(\mathrm{PEG})_{10} \text { monooleate }+\mathrm{NH}_{4}\right]^{+}$were superimposed on the optical image to create the ion maps, depicted in Figure 5. These ion maps show the polyaramid fiber (Figure $5 \mathrm{~A}$ ) and the finish layer (Figure $5 \mathrm{~B}$ ) with surface defects, demonstrating the ability of ambient imaging to investigate finish layers and the detection of defects in finish layers by transient IRMALDESI and LAESI-IMS-TOF-MSI in a single experiment.

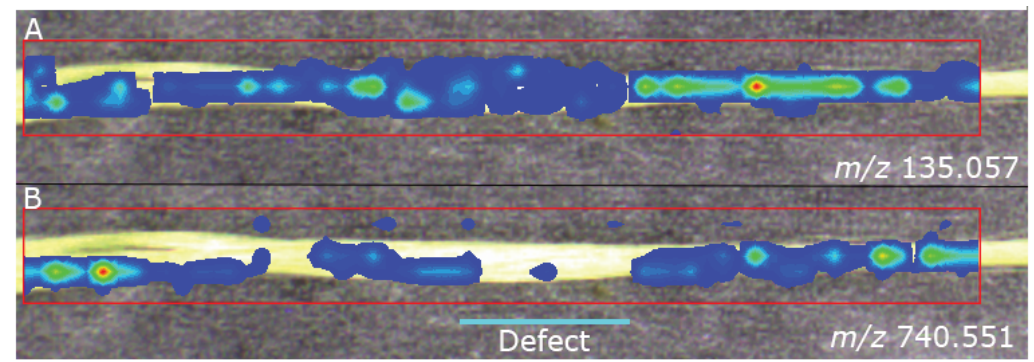

Figure 5. LAESI and IR-MALDESI ion maps of polyaramid fiber with $0.5 \%(\mathrm{~m} / \mathrm{m})$ PEG 400 monooleate finish applied as described in the Experimental Section and an artificial fabricated surface defect, indicated with a cyan line. Part $A$ displays the ion map of one of the fiber fragments $(\mathrm{m} / \mathrm{z} 135.057 \pm 0.001)$, while part $\mathrm{B}$ displays an ion from the finish distribution $\left[(\mathrm{PEG})_{10} \text { monooleate }+\mathrm{NH}_{4}\right]^{+}(\mathrm{m} / \mathrm{z} 740.551 \pm 0.001)$. 


\section{Conclusion}

This study investigated polymer fingerprinting, finish characterization, and the detection of surface defects by mass spectrometry imaging (MSI) under ambient conditions without any sample pretreatment. It was shown that the laser at a wavelength of $2.94 \mu \mathrm{m}$ ablates solid polymer material containing aliphatic and aromatic nitrogen groups, providing fragments to successfully identify PA 6, PA 46, PA 66, PA 12, polyaramid, and M5 polymer species. It was also shown that the finish layer could be fully characterized and, by MSI, localized, which provides detailed insight into surface defects and thus weak spots in the material. Further research might explore possibilities of other laser wavelengths, such as 3.43 $\mu \mathrm{m}$ light to interact with the aliphatic $\mathrm{CH}$ stretch vibration to broaden the polymer application range.

\section{Acknowledgements}

The authors would like to thank Dr. Anton Schotman and Dr. Pieter de Lange for providing synthetic fibers. This research receives funding from The Netherlands Organization for Scientific Research (NWO) in the framework of the Technology Area TA-COAST2 of the Fund New Chemical Innovations (Project No. 053.21.111). 


\section{References}

1 Luo, S.; van Ooij, W. J. J. Adhes. Sci. Technol. 2002, 16, 1715-1735.

2

3

4

5

Drzal, L. T.; Madhukar, M. J. Mater. Sci. 1993, 28, 569-610.

Zhang, X.; Chen, P.; Yu, Q., et al. Vacuum 2013, 97, 1-8.

Chen, J.; Zhu, Y.; Ni, Q., et al. Appl. Surf. Sci. 2014, 321, 103-108.

Niestegge, R. Chem. Fibers Int. 2001, 51, 450-451.

de Lange, P. J.; Akker, P. G.; Maas, A. J. H., et al. Surf. Interface Anal. 2001, 31, 1079-1084.

de Lange, P. J.; Akker, P. G. J. Adhes. Sci. Technol. 2012, 26, 827-839.

Peng, T.; Cai, R.; Chen, C., et al. J. Macromol. Sci. B 2012, 51, 538-550.

de Lange, P. J.; Akker, P. G.; Mäder, E., et al. Compos. Sci. Technol. 2007, 67, 2027-2035.

Weigel, J. Chem. Fibers Int. 2010, 60, 230-233.

Paine, M. R. L.; Barker, P. J.; Blanksby, S. J. Anal. Chim. Acta 2014, 808, 70-82.

Crotty, S.; Gerişlioğlu, S.; Endres, K. J., et al. Anal. Chim. Acta 2016, 932, 1-21.

Bletsos, I. V.; Hercules, D. M.; Greifendorf, D.; Benninghoven, A. Anal. Chem. $1985,57,2384-2388$.

Reddy, S. S.; Dong, X.; Murgasova, R., et al. Macromolecules 1999, 32, 13671374.

Burrell, M. C.; Chao, H. S. I.; Meerman, T. P.; Peterson, G. S. Surf. Interface Anal. 1997, 25, 799-803.

Kang, H. M.; Yoon, T. H.; van Ooij, W. J. J. Adhes. Sci. Technol. 2006, 20, 11551169.

Valantin, C.; Benoit, R.; D, M. P., et al. Appl. Surf. Sci. 2016, 360, Part B, 623633.

Zhou, C.; Li, M.; Garcia, R., et al. Anal. Chem. 2012, 84, 10085-10090.

de Lange, P. J.; Mahy, J. W. G. Fresen. J. Anal. Chem. 1995, 353, 487-493.

Yip, J.; Chan, K.; Sin, K. M.; Lau, K. S. Appl. Surf. Sci. 2003, 205, 151-159.

Biganska, O.; Darque-Ceretti, E.; Giulieri, F.; Combarieu, R. Surf. Interface Anal. 2001, 31, 847-855.

Malshe, P.; Mazloumpour, M.; El-Shafei, A.; Hauser, P. Surf. Coat. Technol. 2013, $217,112-118$.

Yip, J.; Chan, K.; Sin, K. M.; Lau, K. S. Polym. Int. 2004, 53, 627-633.

Wu, S. R.; Sheu, G. S.; Shyu, S. S. J. Appl. Polym. Sci. 1996, 62, 1347-1360.

Lineton, W. B.; Nunn, N. S.; Bishop, D. P. J. Text. I. 1999, 90, 385-394.

Pelster, A.; Körsgen, M.; Kurosawa, T., et al. Anal. Chem. 2016, 88, 9638-9646.

Nielen, M. W. F. Mass Spectrom. Rev. 1999, 18, 309-344.

Scarff, C. A.; Snelling, J. R.; Knust, M. M., et al. J. Am. Chem. Soc. 2012, 134, 9193-9198.

Shan, L.; Murgasova, R.; Hercules, D. M.; Houalla, M. J. Mass Spectrom. 2001, 36, 140-144.

Hilton, G. R.; Jackson, A. T.; Thalassinos, K.; Scrivens, J. H. Anal. Chem. 2008, 80, 9720-9725.

Montaudo, G.; Samperi, F.; Montaudo, M. S. Prog. Polym. Sci. 2006, 31, 277-357.

Krueger, K.; Terne, C.; Werner, C., et al. Anal. Chem. 2013, 85, 4998-5004.

Gies, A. P.; Kliman, M.; McLean, J. A.; Hercules, D. M. Macromolecules 2008, 41, 8299-8301.

Cody, R. B.; Laramée, J. A.; Durst, H. D. Anal. Chem. 2005, 77, 2297-2302.

Rothenbacher, T.; Schwack, W. Rapid Commun. Mass Spectrom. 2009, 23, 2829-

2835.

Rothenbacher, T.; Schwack, W. Rapid Commun. Mass Spectrom. 2010, 24, 21-29. Mess, A.; Vietzke, J.-P.; Rapp, C.; Francke, W. Anal. Chem. 2011, 83, 7323-7330. 
Ackerman, L. K.; Noonan, G. O.; Begley, T. H. Food Addit. Contam. 2009, 26, 1611-1618.

Haunschmidt, M.; Klampfl, C. W.; Buchberger, W.; Hertsens, R. Analyst 2010, 135, 80-85.

Fouyer, K.; Lavastre, O.; Rondeau, D. Anal. Chem. 2012, 84, 8642-8649. Zhang, N.; Zhou, Y.; Zhen, C., et al. Analyst 2012, 137, 5051-5056.

Salter, T. L.; Gilmore, I. S.; Bowfield, A., et al. Anal. Chem. 2013, 85, 1675-1682. Jecklin, M. C.; Gamez, G.; Zenobi, R. Analyst 2009, 134, 1629-1636.

Takáts, Z.; Wiseman, J. M.; Gologan, B.; Cooks, R. G. Science 2004, 306, 471473. 2007, 21, 1693-1704.

47 Friia, M.; Legros, V.; Tortajada, J.; Buchmann, W. J. Mass Spectrom. 2012, 47, 1023-1033. Jackson, A. T.; Williams, J. P.; Scrivens, J. H. Rapid Commun. Mass Spectrom. 2006, 20, 2717-2727.

50 Huang, M.-Z.; Hsu, H.-J.; Wu, C.-I., et al. Rapid Commun. Mass Spectrom. 2007, 21, 1767-1775. Spectrom. 2013, 27, 2595-2600.

54 Vaikkinen, A.; Shrestha, B.; Koivisto, J., et al. Rapid Commun. Mass Spectrom. 2014, 28, 2490-2496. 831-836.

57 Lanucara, F.; Holman, S. W.; Gray, C. J.; Eyers, C. E. Nat. Chem. 2014, 6, 281294.

58 Klun, U.; Andrenšek, S.; Kržan, A. Polymer 2001, 42, 7095-7099.

59 Montaudo, G.; Montaudo, M. S.; Puglisi, C.; Samperi, F. J. Polym. Sci., Part A: Polym. Chem. 1996, 34, 439-447.

60 Bahr, U.; Lüderwald, I.; Müller, R.; Schulten, H. R. Angew. Makromol. Chem. 1984, $120,163-175$.

61 Schulten, H.-R.; Plage, B. J. Polym. Sci., Part A: Polym. Chem. 1988, 26, 23812394.

62 Schulten, H. R.; Plage, B.; Ohtani, H.; Tsuge, S. Angew. Makromol. Chem. 1987, $155,1-20$.

63 Kappes, R. S.; Schönfeld, F.; Li, C., et al. SpringerPlus 2014, 3, 489. 


\section{Supporting Information Chapter 2}

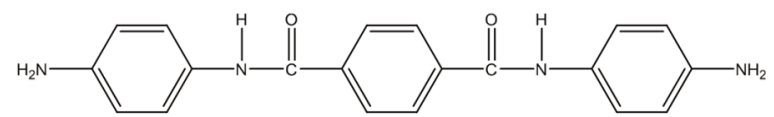

Figure S1. Chemical structure of aramid trimer- $\mathrm{NH}_{2}$.

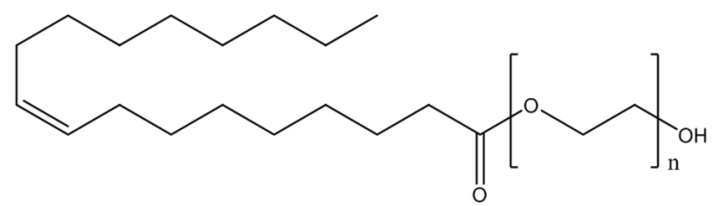

Figure S2. Chemical structure of the used finish on polyaramid fiber (PEG 400 monooleate).

\begin{tabular}{lcccc}
\hline Polymer & $m / z$ experimental & $\begin{array}{c}\text { proposed elemental } \\
\text { composition }\end{array}$ & $m / z$ theory & $\Delta \mathrm{mDa}$ \\
\hline PA 6 & 114.091 & {$\left[\mathrm{C}_{6} \mathrm{H}_{12} \mathrm{NO}^{+}\right.$} & 114.092 & 1 \\
\hline PA 12 & 198.188 & {$\left[\mathrm{C}_{12} \mathrm{H}_{24} \mathrm{NO}^{+}\right.$} & 198.186 & 2 \\
\hline PA 46 & 115.089 & {$\left[\mathrm{C}_{5} \mathrm{H}_{11} \mathrm{~N}_{2} \mathrm{O}\right]^{+}$} & 115.087 & 2 \\
& 128.105 & {$\left[\mathrm{C}_{7} \mathrm{H}_{14} \mathrm{NO}^{+}\right.$} & 128.108 & 2 \\
& 142.126 & {$\left[\mathrm{C}_{8} \mathrm{H}_{16} \mathrm{NO}\right]^{+}$} & 142.123 & 3 \\
\hline PA 66 & 156.138 & {$\left[\mathrm{C}_{9} \mathrm{H}_{18} \mathrm{NO}^{+}\right.$} & 156.139 & 1 \\
& 128.105 & {$\left[\mathrm{C}_{7} \mathrm{H}_{14} \mathrm{NO}\right]^{+}$} & 128.108 & 2 \\
& 142.121 & {$\left[\mathrm{C}_{8} \mathrm{H}_{16} \mathrm{NO}\right]^{+}$} & 142.123 & 2 \\
& 156.138 & {$\left[\mathrm{C}_{9} \mathrm{H}_{18} \mathrm{NO}\right]^{+}$} & 156.139 & 1 \\
\hline & 170.153 & {$\left[\mathrm{C}_{10} \mathrm{H}_{20} \mathrm{NO}^{+}\right.$} & 170.155 & 2 \\
\hline
\end{tabular}

Table S1. Mass accuracy for proposed elemental compositions presented in Figure 2. 


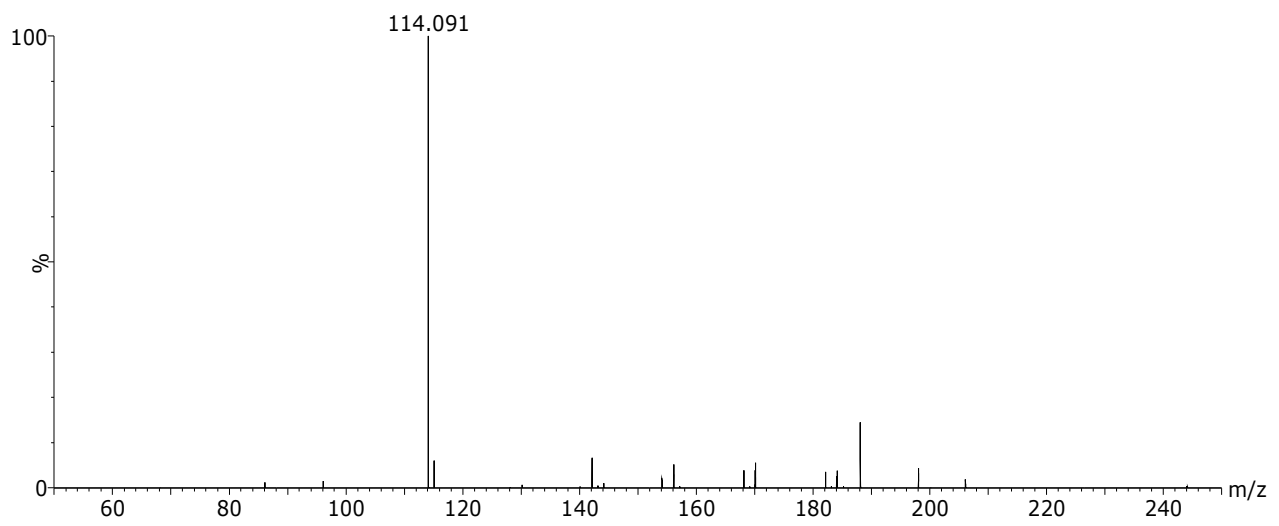

Figure S3. LAESI-MS background subtracted mass spectrum of PA $6\left(M_{w} 15 \times 10^{3}\right)$ polymer pellets.

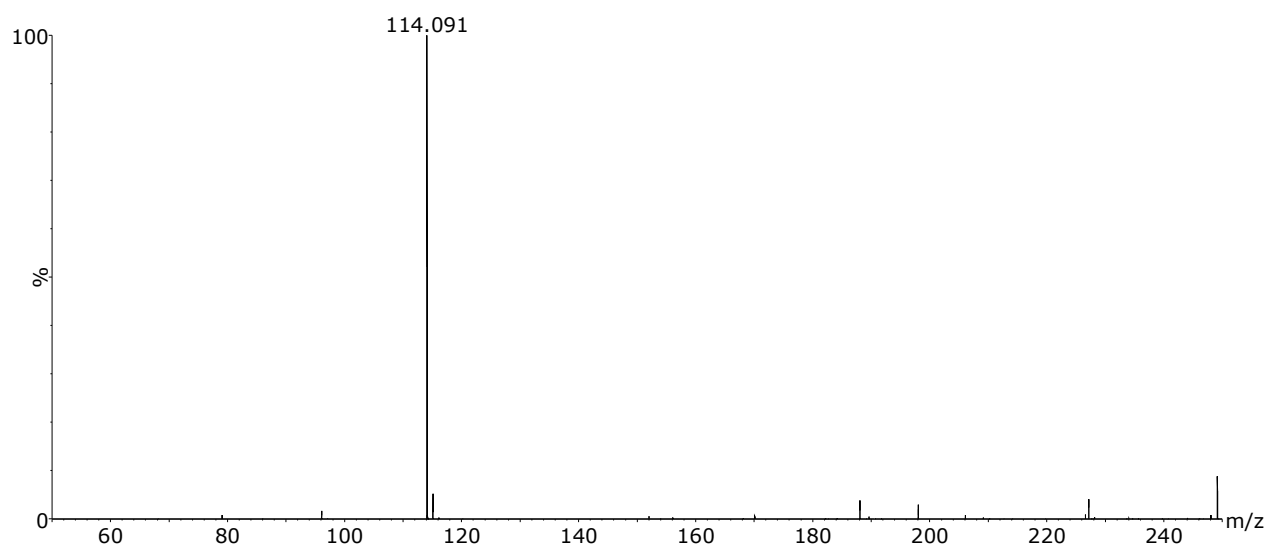

Figure S4. LAESI-MS background subtracted mass spectrum of PA $6\left(M_{w} 30 \times 10^{3}\right)$ polymer pellets. 


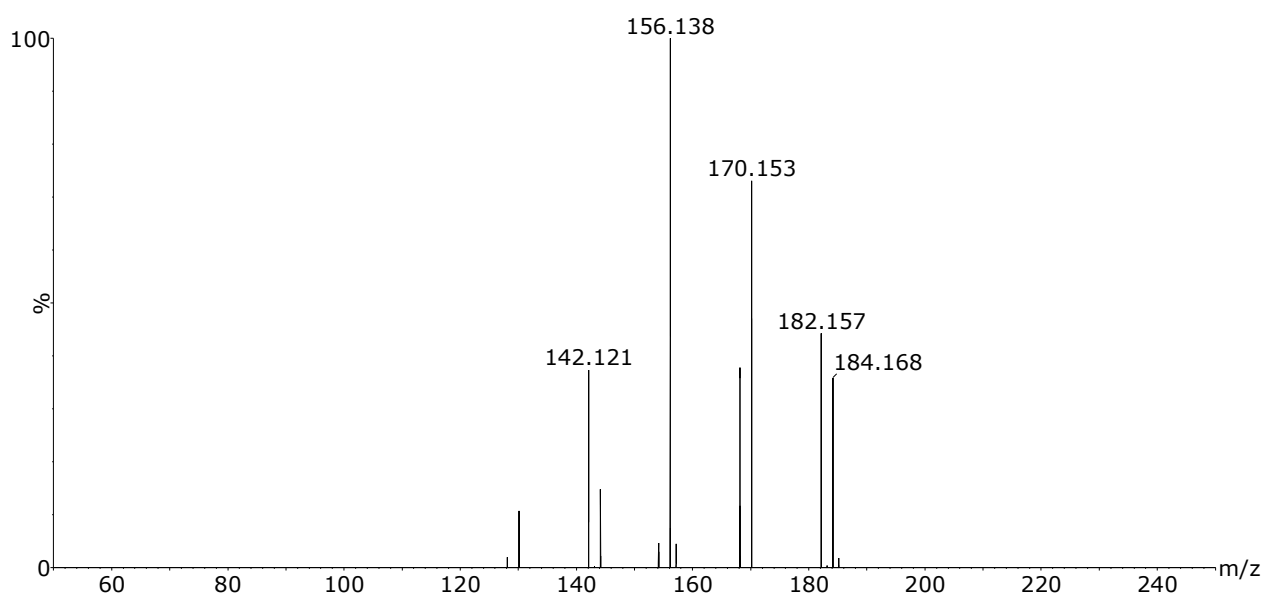

Figure S5. LAESI-MS background subtracted mass spectrum of PA $66\left(\mathrm{M}_{\mathrm{w}} 33 \times 10^{3}\right)$ synthetic fiber.

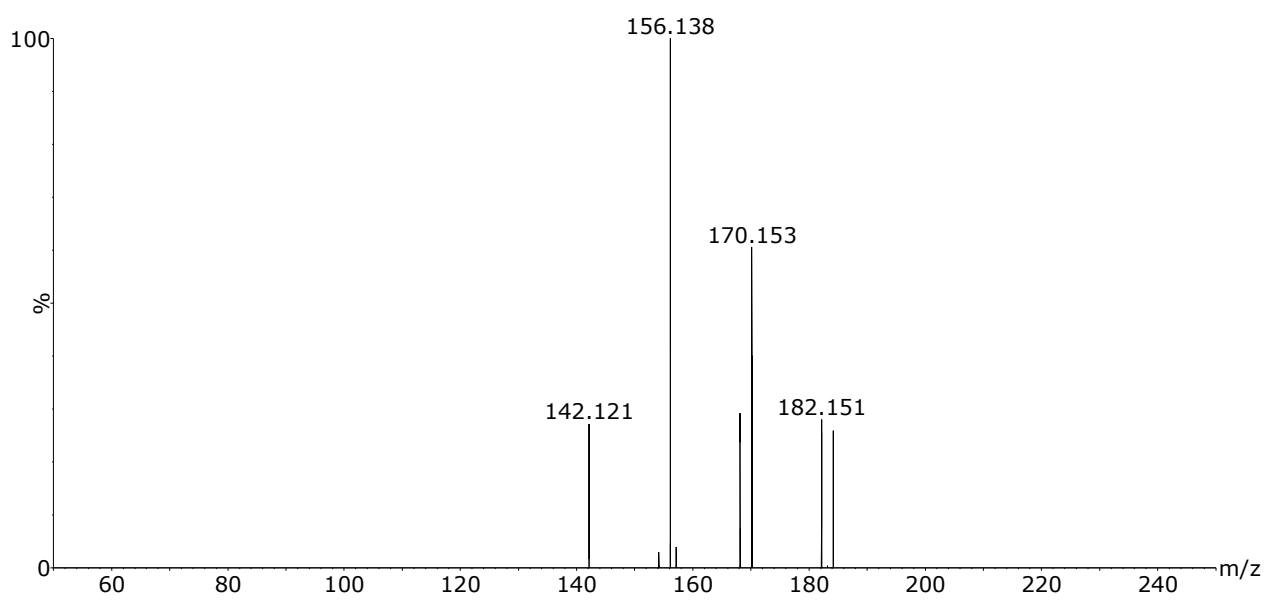

Figure S6. LAESI-MS background subtracted mass spectrum of PA $66\left(M_{w} 46 \times 10^{3}\right)$ synthetic fiber.

\section{Method of Extraction and ESI-MS Parameters}

$10 \mathrm{~cm}$ of polyaramid fiber was extracted for 30 seconds with $4 \mathrm{~mL} \mathrm{MeOH}$. Thereafter, $2 \mathrm{~mL}$ extract was added to $2 \mathrm{~mL} \mathrm{H}_{2} \mathrm{O}+0.2 \% \mathrm{FA}+80 \mathrm{ng} / \mathrm{mL}$ leu-enk to obtain the same $\mathrm{ESI}$ solution as used in LAESI experiments. The ESI function in our LAESI setup was used for all ESI experiments, all parameters were set as used in LAESI-MS experiments, but without engaging the laser. 
Ambient Characterization of Synthetic Fibers by LAESI-MS

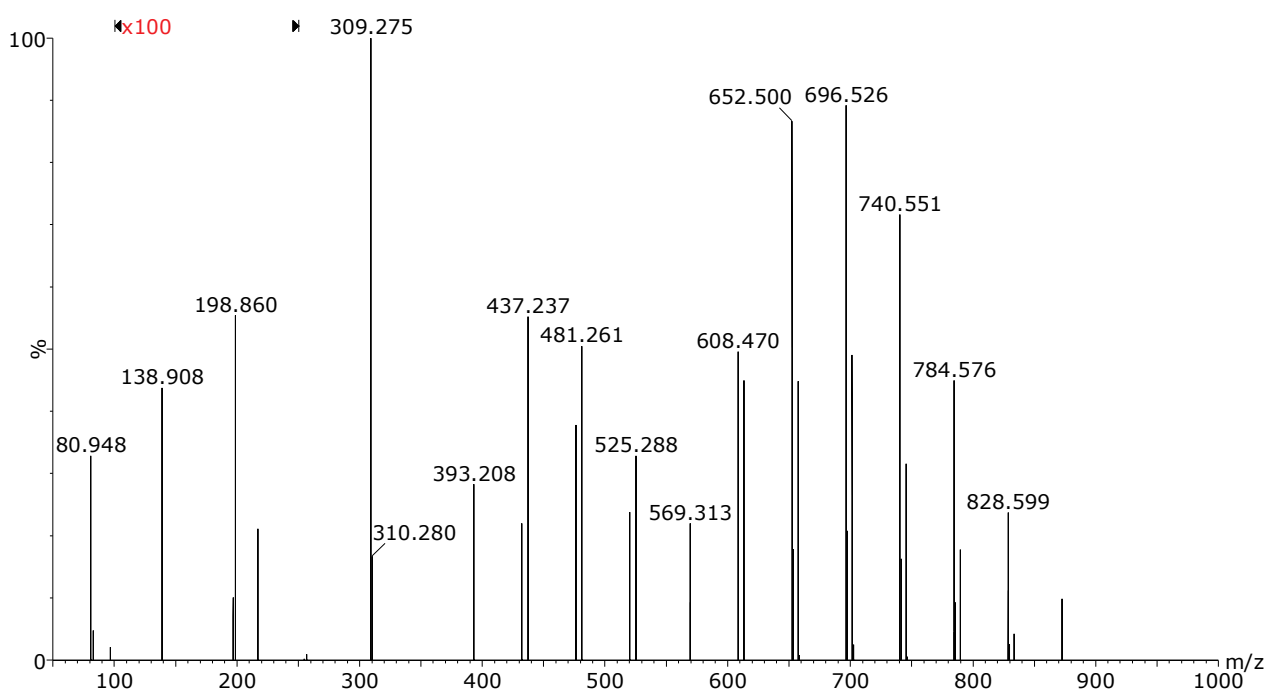

Figure S7. ESI-MS background subtracted mass spectrum of polyaramid fiber extract. The intensity of $\mathrm{m} / \mathrm{z}$ range 100 to 250 is magnified by a factor 100 . 


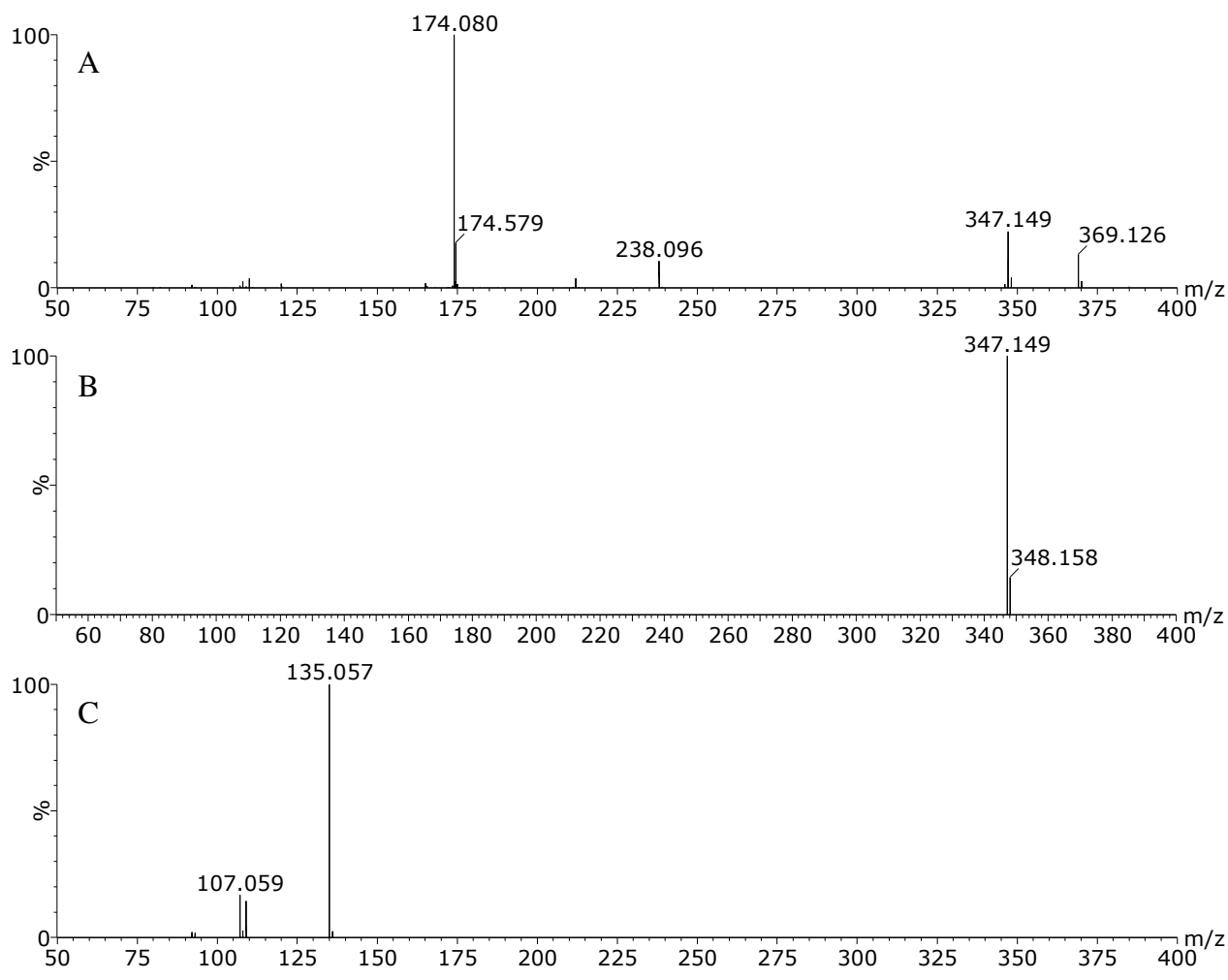

Figure S8. Part A displays the ESI-MS background subtracted mass spectrum of aramid trimer- $\mathrm{NH}_{2}, 1 \mu \mathrm{g} / \mathrm{mL}$ solution in $\mathrm{MeOH}-\mathrm{H}_{2} \mathrm{O}(1: 1)$ with $0.1 \%$ FA. Part $\mathrm{B}$ displays the LAESI-MS background subtracted mass spectrum of aramid trimer$\mathrm{NH}_{2}, 10 \mu \mathrm{g} / \mathrm{mL}$ solution in $\mathrm{MeOH}-\mathrm{H}_{2} \mathrm{O}(1: 1)$, and part $\mathrm{C}$ displays the LAESI-MS background subtracted mass spectrum of aramid trimer- $\mathrm{NH}_{2}$ powder. 


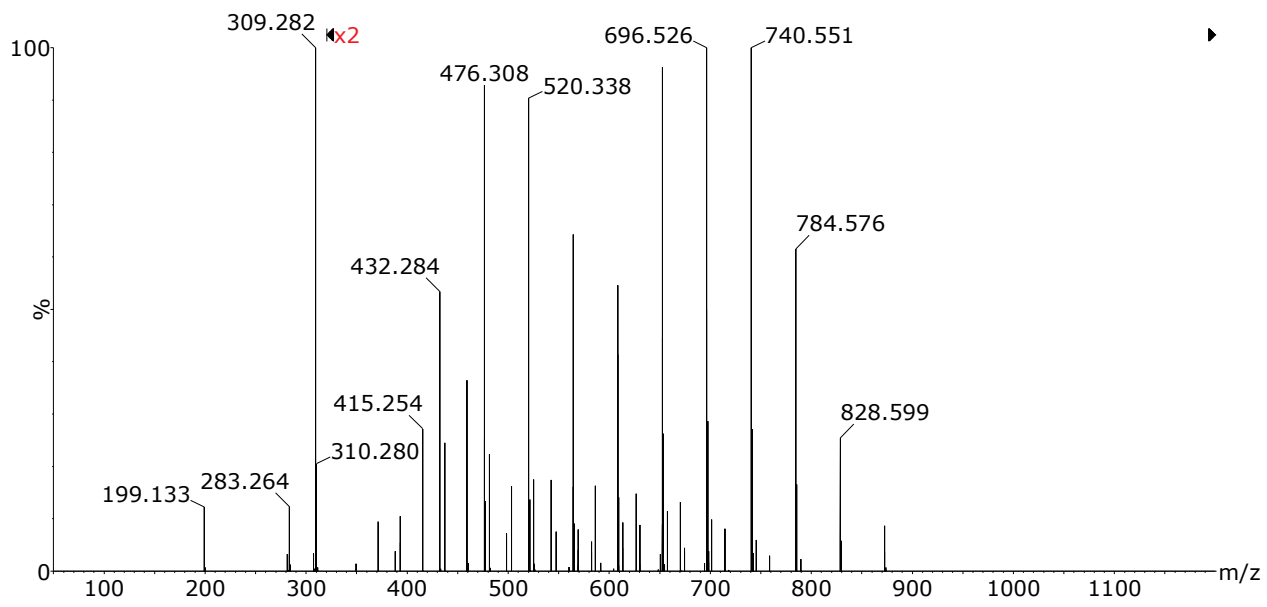

Figure S9A. IR-MALDESI-IMS-MS background subtracted mass spectrum of $0.5 \%(\mathrm{~m} / \mathrm{m})$ PEG 400 monooleate on polyaramid fiber. The intensity of $m / z$ range 325 to 1200 is magnified by a factor 2 .

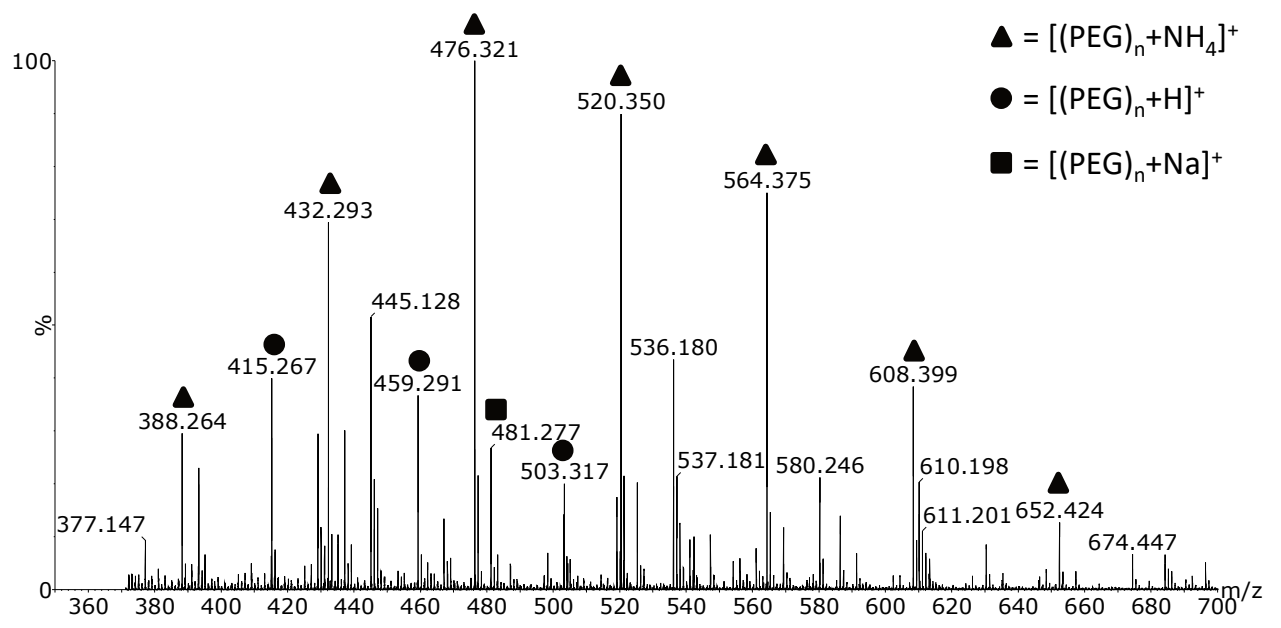

Figure S9B. IR-MALDESI-IMS-MS selected (area 1 in Figure 4) polyethylene glycol distribution as part of the PEG 400 monooleate finish on polyaramid fiber. 


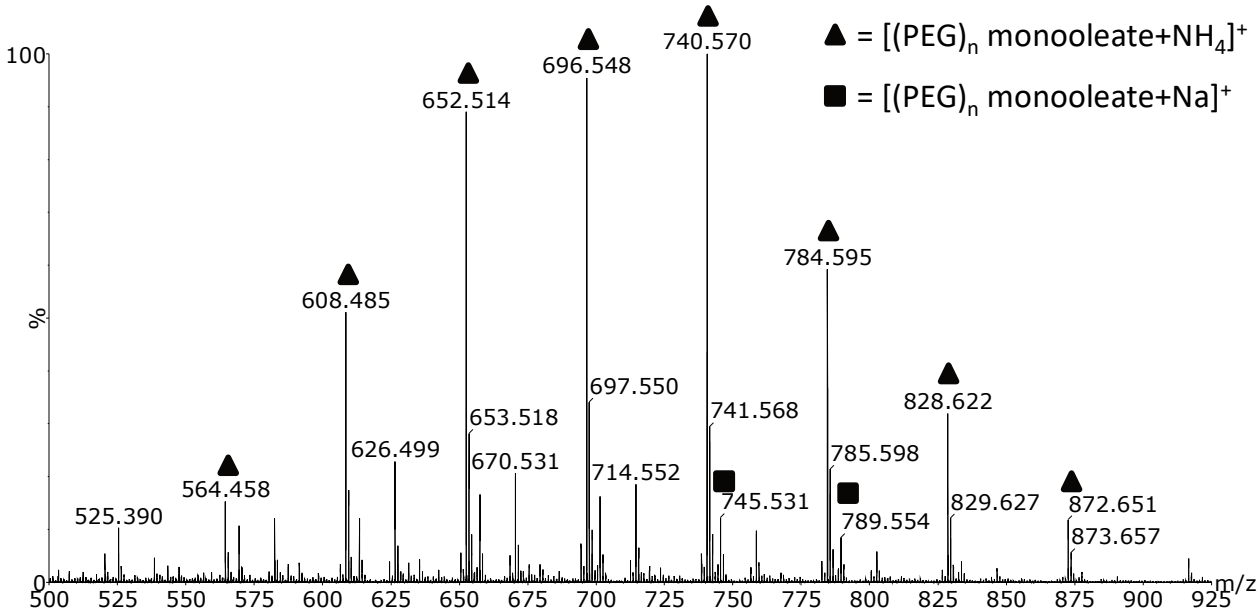

Figure S9C. IR-MALDESI-IMS-MS selected (area 2 in Figure 4) polyethylene glycol monooleate distribution as part of the PEG 400 monooleate finish on polyaramid fiber.

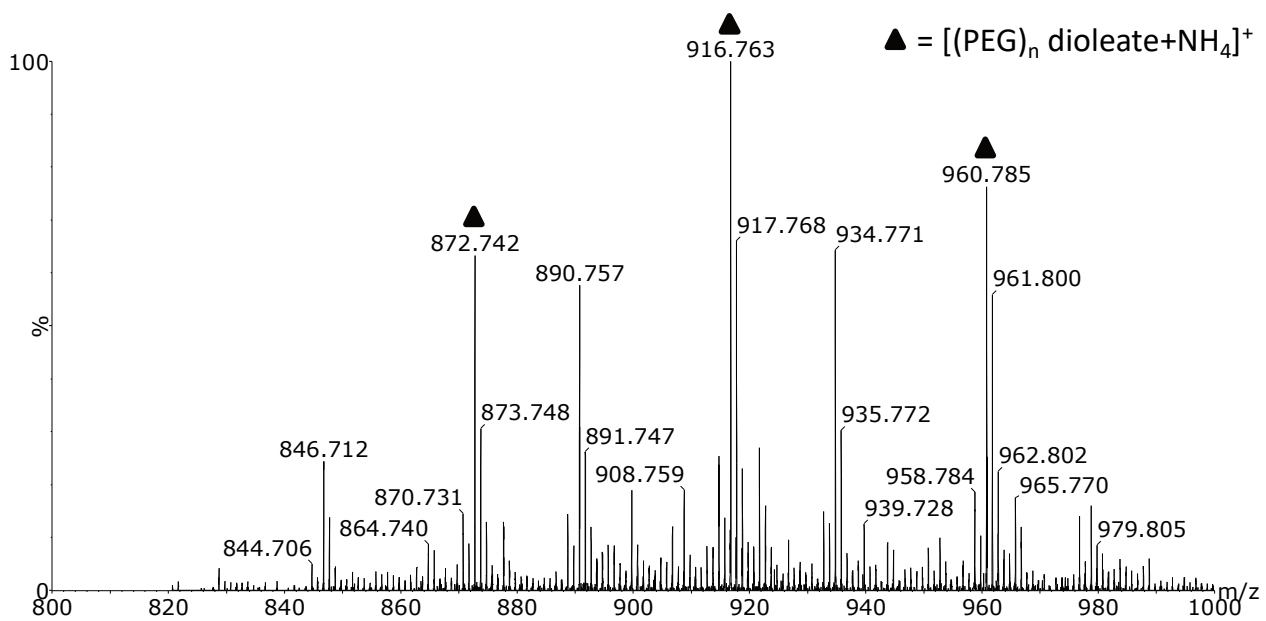

Figure S9D. IR-MALDESI-IMS-MS selected (area 3 in Figure 4) polyethylene glycol dioleate distribution as part of the PEG 400 monooleate finish on polyaramid fiber.<smiles>[CH2+]C=COC(=O)CCCCCCC/C=C\CC</smiles>

Figure S10. Proposed chemical structure of $m / z 309.282$. 


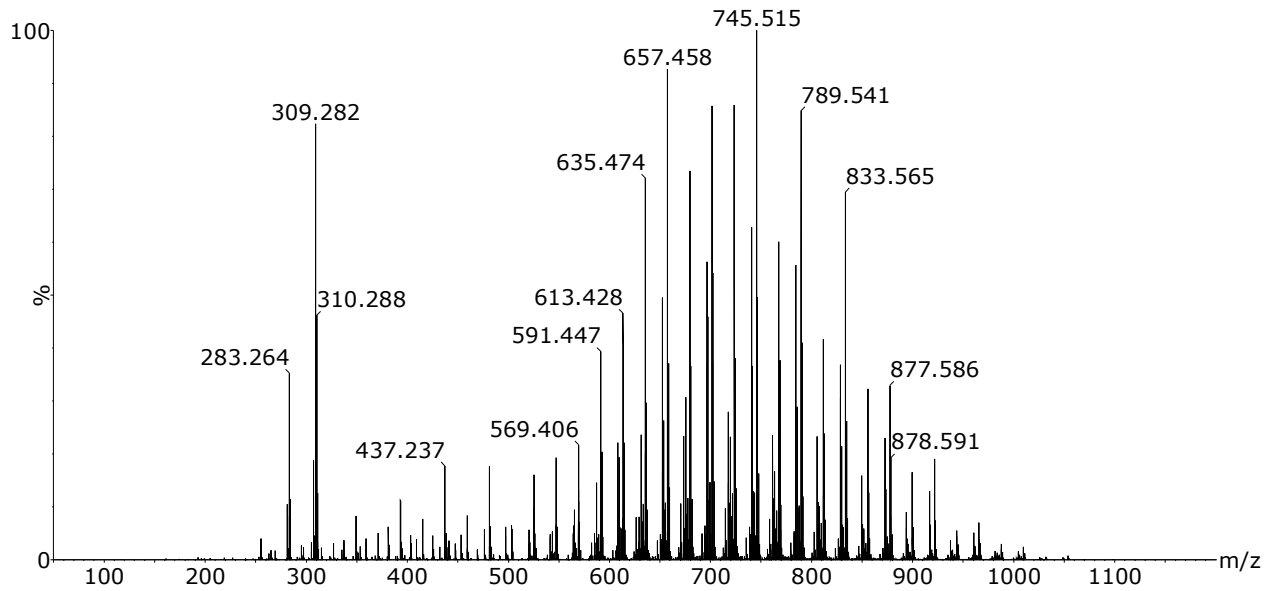

Figure S11A. LAESI-IMS-MS background subtracted mass spectrum of a 1\% (v/v) solution of PEG 400 monooleate in $\mathrm{MeOH}$.

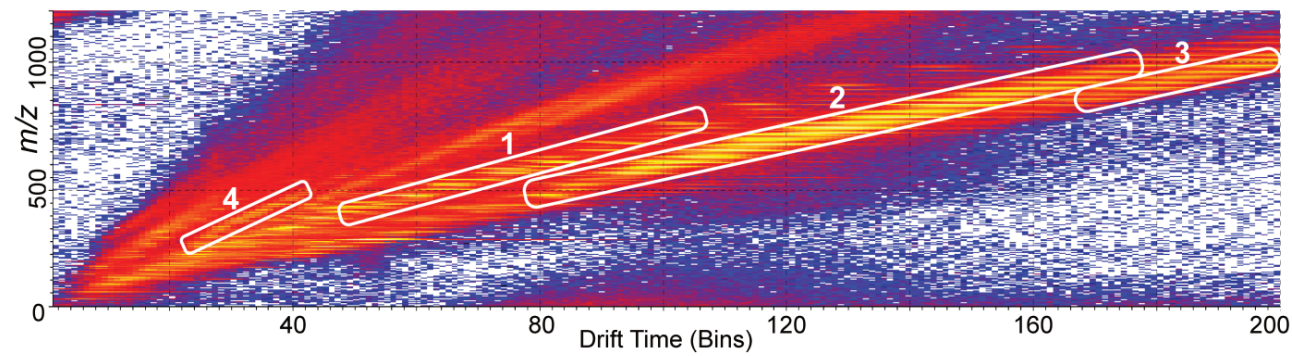

Figure S11B. LAESI-IMS-MS 2D map of a $1 \%(\mathrm{v} / \mathrm{v})$ solution of PEG 400 monooleate in $\mathrm{MeOH}$. Four areas were isolated and the corresponding mass spectra are given for area 1 in Figure S11C, 2 in Figure S11D, 3 in Figure S11E and 4 in Figure S11F. 


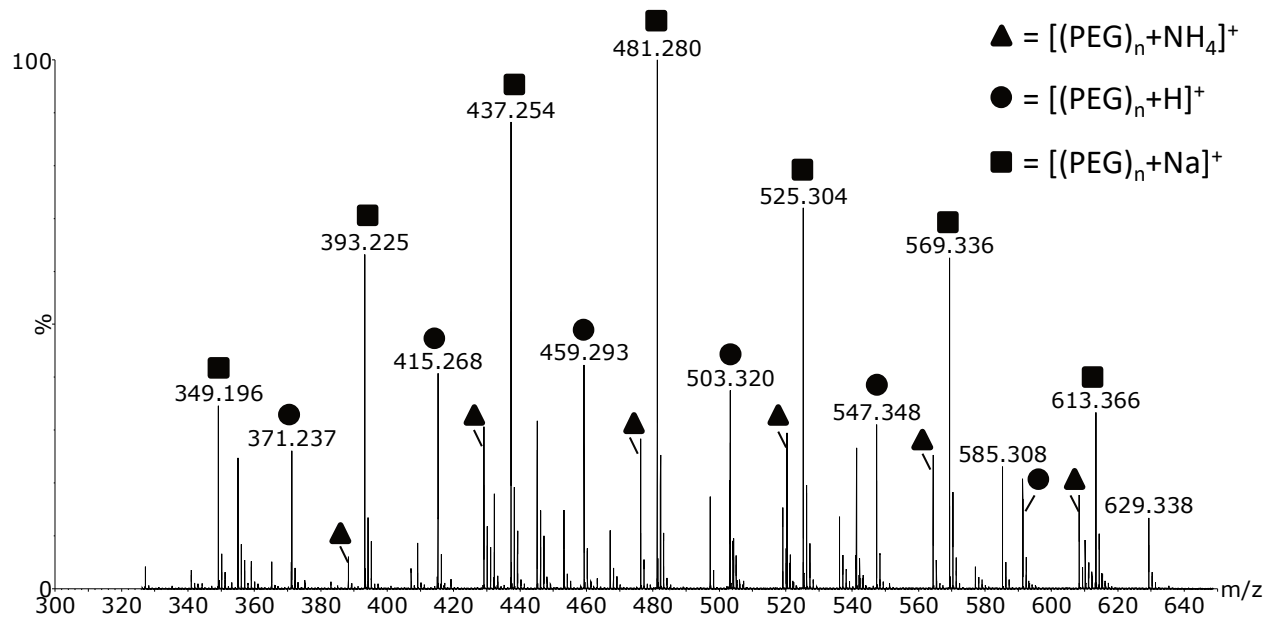

Figure S11C. LAESI-IMS-MS selected (area 1 in Figure S11B) polyethylene glycol distribution as part of a 1\% (v/v) solution of PEG 400 monooleate in $\mathrm{MeOH}$.

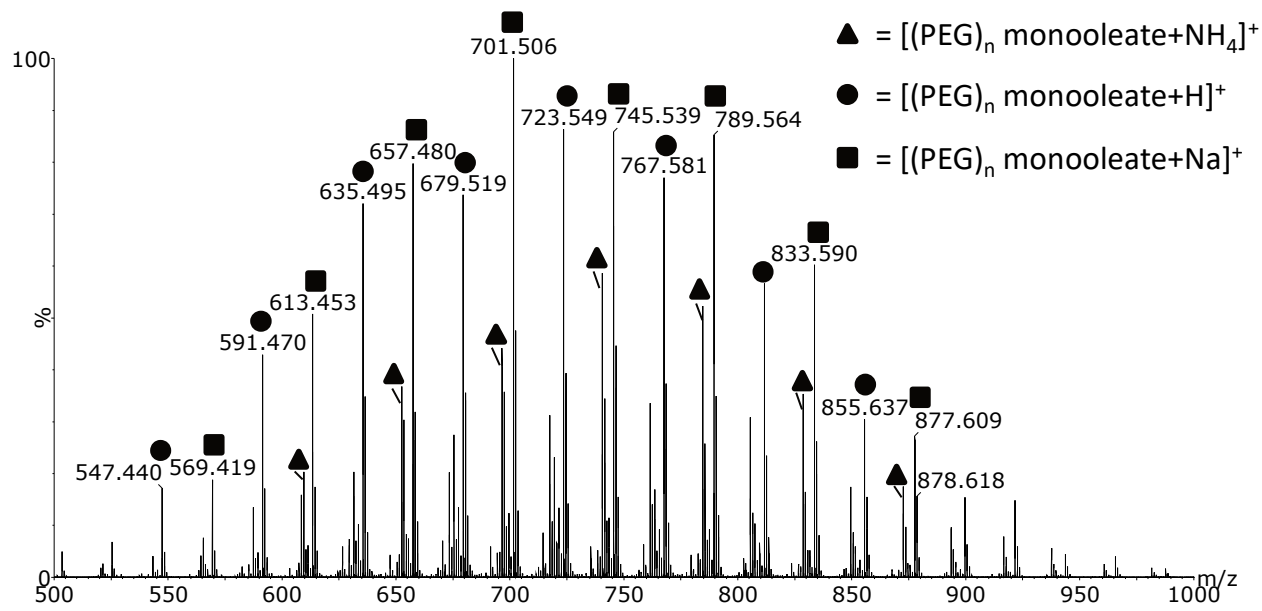

Figure S11D. LAESI-IMS-MS selected (area 2 in Figure S11B) polyethylene glycol monooleate distribution as part of a $1 \%(\mathrm{v} / \mathrm{v})$ solution of PEG 400 monooleate in $\mathrm{MeOH}$. 


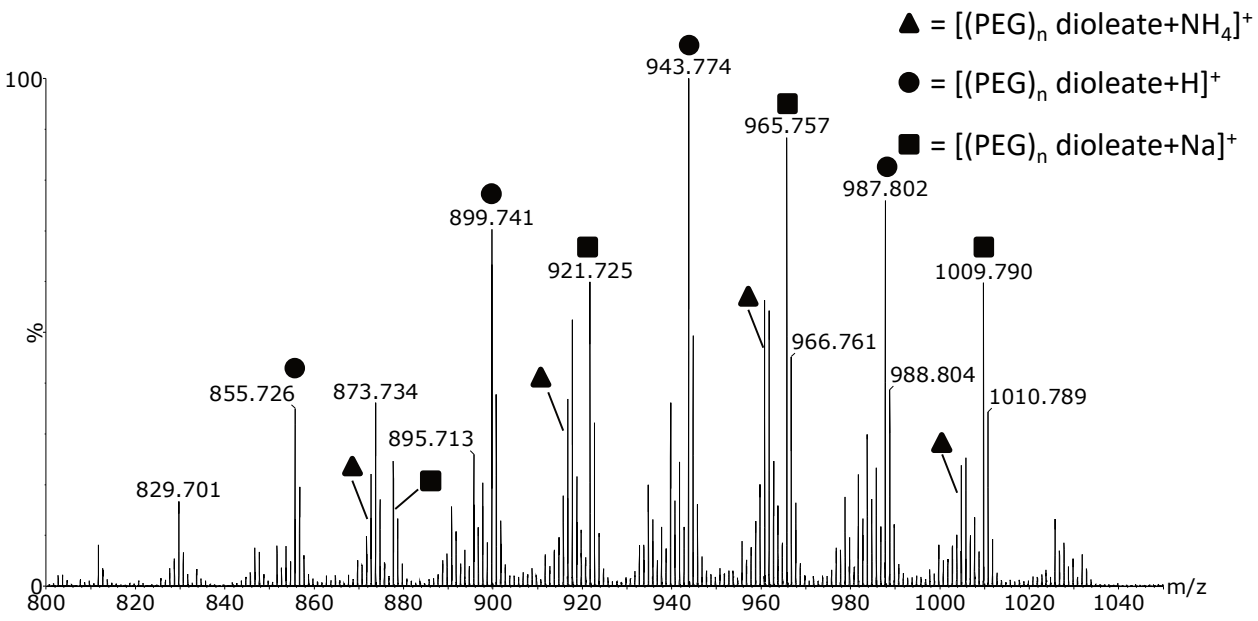

Figure S11E. LAESI-IMS-MS selected (area 3 in Figure S11B) polyethylene glycol dioleate distribution as part of a $1 \%$ (v/v) solution of PEG 400 monooleate in $\mathrm{MeOH}$.

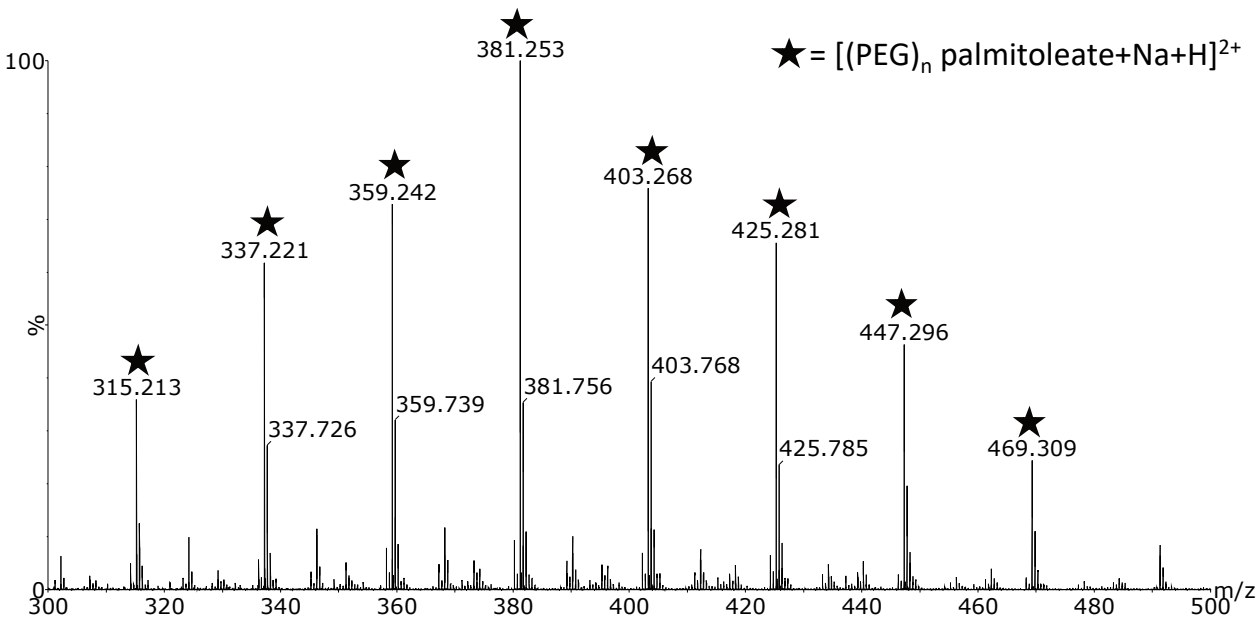

Figure S11F. LAESI-IMS-MS selected (area 4 in Figure S11B) double charged polyethylene glycol palmitoleate distribution as part of a $1 \%(\mathrm{v} / \mathrm{v})$ solution of PEG 400 monooleate in $\mathrm{MeOH}$. 


\section{Chapter 3}

\section{Reactive Laser Ablation Electrospray Ionization Time-Resolved Mass Spectrometry of Click Reactions}

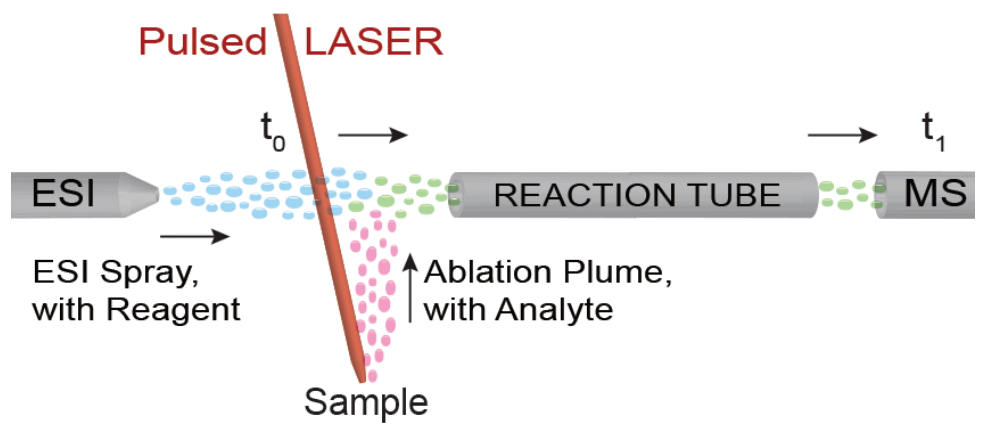

This Chapter was published as:

Fred A. M. G. van Geenen, Maurice C. R. Franssen, Han Zuilhof and Michel W. F. Nielen. Reactive Laser Ablation Electrospray Ionization Time-Resolved Mass Spectrometry of Click Reactions. Analytical Chemistry, 2018, 90, 10409-10416. https://pubs.acs.org/doi/abs/10.1021/acs.analchem.8b02290 


\section{Abstract}

Reactions in confined compartments like charged microdroplets are of increasing interest, notably because of their substantially increased reaction rates. When combined with ambient ionization mass spectrometry (MS), reactions in charged microdroplets can be used to improve the detection of analytes or to study the molecular details of the reactions in real time. Here, we introduce a reactive laser ablation electrospray ionization (reactive LAESI) time-resolved mass spectrometry (TRMS) method to perform and study reactions in charged microdroplets. We demonstrate this approach with a class of reactions new to reactive ambient ionization MS: so-called click chemistry reactions. Click reactions are high-yielding reactions with a high atom efficiency, and are currently drawing significant attention from fields ranging from bioconjugation to polymer modification. Although click reactions are typically at least moderately fast (time scale of minutes to a few hours), in a reactive LAESI approach a substantial increase of reaction time is required for these reactions to occur. This increase was achieved using microdroplet chemistry and followed by MS using the insertion of a reaction tube - up to $1 \mathrm{~m}$ in length - between the LAESI source and the MS inlet, leading to near complete conversions due to significantly extended microdroplet lifetime. This novel approach allowed for the collection of kinetic data for a model (strain-promoted) click reaction between a substituted tetrazine and a strained alkyne and showed in addition excellent instrument stability, improved sensitivity, and applicability to other click reactions. Finally, the

methodology was also demonstrated in a mass spectrometry imaging setting to show its feasibility in future imaging experiments. 


\section{Introduction}

Mass spectrometry (MS), stand-alone or hyphenated with separation techniques like chromatography or electrophoresis, is a well-established analysis technique in industry, healthcare, and many fields of science, such as organic synthesis, ${ }^{1}$ metabolomics, ${ }^{2}$ and proteomics. $^{3}$ The abundant use of MS and its hyphenations is mainly due to the combination of high sensitivity, selectivity, speed, and capabilities for structure elucidation by MS ${ }^{n}$. MS can also be optimized to study molecular reactions in solution. ${ }^{4}$ Time-resolved mass spectrometry (TRMS), where reaction vessels are directly coupled with MS analyses, was first introduced by Lee et al. in $1989 .{ }^{5}$ TRMS is used to study dynamic processes in which reagents convert into (by-)products in real time, typically in the microsecond to minute time range. ${ }^{6-12}$ The development and use of TRMS has been reviewed recently by Lento and Wilson. ${ }^{13}$

The discovery of reaction acceleration in charged microdroplets, even up to six orders of magnitude, ${ }^{14}$ by the Cooks and Zare groups brings a new way to perform and study a wide range of (bio)molecular reactions. ${ }^{15,16}$ It has been used in organic synthesis, ${ }^{17-20}$ in order to predict the likely success of up-scaling reactions, ${ }^{21}$ and as a continuous synthesis system. ${ }^{22}$ Other exciting fields of application are its use to study the chemistry of life, ${ }^{23}$ which mostly takes place in small enclosed volumes such as cells or atmospheric aerosols, ${ }^{24-26}$ or to improve detection of analytes in ambient ionization MS. ${ }^{27-32}$ Several explanations have been suggested why reaction acceleration in charged microdroplets may occur: partial desolvation of reagents at the droplet-to-air interface, ${ }^{32-37} \mathrm{pH}$ change, ${ }^{38}$ and/or a potentially increased reagent concentration associated with solvent evaporation. ${ }^{20}$

The combination of TRMS and charged microdroplets can also be used to determine reaction kinetics, provided that reaction times can be accurately measured. Previously, reaction times with electrospray ionization (ESI) over distances up to $100 \mathrm{~cm}$ were estimations based on approximate droplet flight time, assuming that droplets are carried along by the nebulizing gas. ${ }^{39}$ Further steps have been taken to record reaction time with desorption electrospray ionization (DESI) ${ }^{40}$ and microdroplet fusion in extractive electrospray ionization $(E E S I)^{41}$ by using Doppler techniques to measure the droplet velocity on the $\mu$ s timescale. ${ }^{42,43}$ With DESI the droplet velocity was decreasing quickly with distance due to aerodynamic drag. ${ }^{42}$ With EESI, pressurized nebulizing nitrogen gas was used as propulsive force providing more stable average velocities before reaching the MS. ${ }^{43}$ The reaction is essentially stopped when the droplets reach the vacuum of the heated MS interface. ${ }^{39}$ Up to now, a direct way to measure reaction time over distances up to $100 \mathrm{~cm}$ has, to our knowledge, not been described.

Laser ablation electrospray ionization (LAESI) MS provides a way to analyze a wide range of samples - including liquids, ${ }^{44,45}$ solid materials, ${ }^{46}$ tissue,,${ }^{47-51}$ and single cells ${ }^{52-54}$ under ambient conditions. ${ }^{55}$ In LAESI, a pulsed laser directed onto a substrate produces a neutral plume of ablated material, which can be extracted with charged microdroplets 
produced by an electrospray probe for subsequent analyte ionization. When the electrospray solution contains a reagent, LAESI can be used to perform reactions with the ablated compounds in charged microdroplets. A combination with TRMS would enable the determination of reaction kinetics. Alternatively, the reaction could be used to generate chemical derivatives of the ablated compounds to improve the detection of analytes in ambient ionization MS. So far, mainly reactive DESI has been used in order to improve analyte detection by increased proton affinity, ${ }^{28-32}$ adapted analyte $m / z$ value,${ }^{56-60}$ altered polarity, ${ }^{61-63}$ or as a tool for structure elucidation. ${ }^{64}$ Apart from DESI also a few other reactive ambient ionization techniques have been reported. ${ }^{65-69}$ Potentially, similar reagents can also be used in a reactive LAESI system. In current literature however, reactive LAESI exclusively refers to coordination complexes with metal salts like lithium or silver in order to achieve cationization. ${ }^{70,71} \mathrm{~A}$ reason why common DESI reactions have not been reported for LAESI could be the relatively short reaction time window available in LAESI. For a comparable extractive electrospray ionization setup, the reaction time was stated to be in the order of only $15 \mu \mathrm{s} .{ }^{43}$ Thus, a major challenge for molecular reactions in ambient ionization LAESI MS is to significantly increase the reaction time. One possibility to achieve this is the introduction of a reaction tube, as previously used in an ESI study of a Hantzsch reaction. ${ }^{39}$ However, for introduction of a reaction tube into a reactive LAESI setting additional space needs to be available between the electrospray probe and reaction tube in order to allow the laser to irradiate the sample, ablate the material and subsequently extract the plume by electrospraygenerated charged microdroplets. In this experimental setup, temporal resolution can then be obtained in a direct and precise fashion by the time difference between the laser pulse and the MS detection of analytes or reaction products.

In the present work we report a novel ambient ionization MS approach, viz. reactive LAESI in combination with accurate reaction time determination. The method is demonstrated with an inverse electron demand Diels-Alder addition (IEDDA) - click - reaction between substituted tetrazines and a strained alkyne or alkene to form a stable pyridazine product. As a result of the insertion of a reaction tube, the (charged) microdroplet lifetime, and thus reaction time, was increased up to 4-5 orders of magnitude. Two new chemical bonds are formed in this reaction, underlining the progress versus the cationization LAESI experiments mentioned above. Click reactions are drawing significant attention from different fields such as labeling of biomolecules for imaging, ${ }^{72,73}$ bioconjugation, ${ }^{74-77}$ and polymer modification, ${ }^{78}$ due to the high efficiency. Our reactive LAESI methodology successfully showed pseudo-first order kinetic data of a model click reaction system between BCN-amine $\mathbf{1}$ and dipyridyltetrazine $\mathbf{2 a}$, whereas TCO-amine $\mathbf{4}$ and other tetrazines performed equally well (structures provided in Figure 1). Finally, the feasibility of reactive LAESI mass spectrometry imaging (MSI) was demonstrated exploiting the combined potential of laser spatial resolution and microdroplet-induced reaction acceleration. 


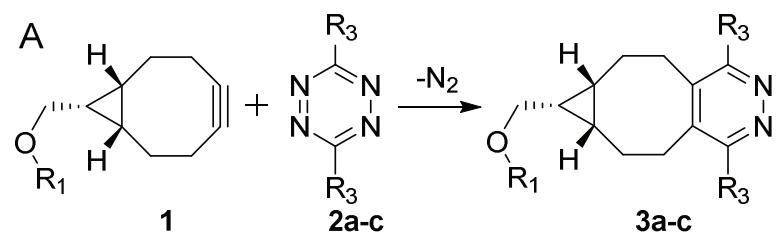<smiles>[R3]C1=NNC([R3])=C2CCCC([R2])CCC12</smiles>

4

$2 a$

$5 \mathbf{a}$

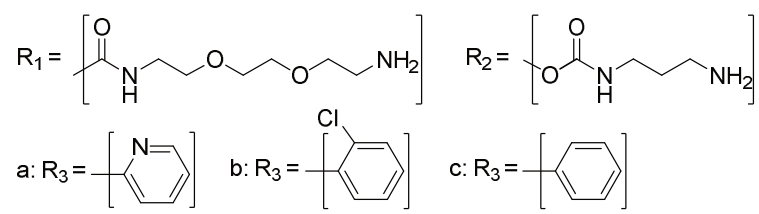

Figure 1. IEDDA reactions between an amine-terminated strained $(A)$ cyclooctyne $(B C N$-amine; 1$)$, or $(B)$ transcyclooctene (TCO-amine; 4) and disubstituted-tetrazines 2 which feature different polarities (dipyridyl-tetrazine 2a, clofentezine $\mathbf{2 b}$, diphenyl-tetrazine $\mathbf{2 c}$ ). The [4+2] cycloaddition initially forms a strained bicyclic reaction intermediate that is converted upon release of $\mathrm{N}_{2}$ into the pyridazine $\mathbf{3}$ or dihydropyridazine $\mathbf{5}$ click reaction product.

\section{Experimental section}

\section{Materials}

Ultrapure water $\left(\mathrm{H}_{2} \mathrm{O}\right), 18.2 \mathrm{M} \Omega \times \mathrm{cm}^{-1}$ at $25^{\circ} \mathrm{C}$, was freshly produced daily with a Millipore (Molsheim, France) Integral 3 system. Methanol (MeOH), LC-MS grade, was purchased from VWR (Leuven, Belgium). $\quad N$-[(1R,8S,9s)-Bicyclo[6.1.0]non-4-yn-9ylmethyloxycarbonyl]-1,8-diamino-3,6-dioxaoctane (BCN-amine, 1), trans-4-cycloocten-1-yl (3-aminopropyl) carbamate (TCO-amine, 4), 3,6-di-2-pyridyl-1,2,4,5-tetrazine (dipyridyltetrazine, 2a), 3,6-di-2-chlorophenyl-1,2,4,5-tetrazine (clofentezine, 2b), 3,6-diphenyl-1,2,4,5tetrazine (diphenyl-tetrazine, 2c), reserpine, and 96 well plates (untreated) were obtained from Sigma-Aldrich (Zwijndrecht, The Netherlands). Chemical structures of reagents are given in Figure 1. All chemicals were used without further purification.

\section{Experimental Setup and Measurement of Reaction Time}

A Protea Biosciences (Morgantown, WV) LAESI DP-1000 system was electronically connected to a Waters (Manchester, U.K.) Synapt G2-S traveling wave ion mobility time of flight mass spectrometer, and used for all analyses. The distance between the LAESI source 
and the MS inlet was varied between 1 and $100 \mathrm{~cm}$ (see Figure 2A). Transport of ions from the LAESI ionization source to the MS inlet was assisted by nitrogen as electrospray nebulizer gas, controlled at $1 \mathrm{~L} \times \mathrm{min}^{-1}$ by a Bronkhorst (Veenendaal, The Netherlands) Mani-Flow mass flow controller, directed toward the inlet of a grounded copper reaction tube - of 1 to $100 \mathrm{~cm}$ long; internal diameter $1.0 \mathrm{~cm}$ (Gamma, The Netherlands) - at room temperature. The nebulizer gas flow was optimized for $50 \mathrm{~cm}$ reaction tube length. Distances between ESI to reaction tube and reaction tube to MS inlet, both under ambient conditions, were 18 and 20 $\mathrm{mm}$, respectively. A picture of the experimental setup is shown in Figure S1. LAESI desktop software v.2.0.1.3 (Protea Biosciences) was used to control experimental parameters of the LAESI system. The Nd:YAG optical parametric oscillator mid infrared laser $(2.94 \mu \mathrm{m})$ was set to $100 \%$ laser power $\left(\Phi 3.2 \mathrm{~J} / \mathrm{cm}^{2}\right)$ and 10 pulses with a specified pulse length of 5 ns were acquired on every spot on the 96-well plate at a frequency of $10 \mathrm{~Hz}$. The 96-well plate contained $383 \mu \mathrm{L}$ of $100 \mu \mathrm{M}$ dipyridyl-tetrazine $2 \mathbf{a}$ in $\mathrm{MeOH}-\mathrm{H}_{2} \mathrm{O}(1: 1)$ per well and the temperature-controlled sample stage was cooled to $4{ }^{\circ} \mathrm{C}$ to avoid evaporation during analysis. A solution of $100 \mu \mathrm{M} \mathrm{BCN}$-amine 1 in $\mathrm{MeOH}-\mathrm{H}_{2} \mathrm{O}$ (1:1) was used as electrospray solvent at a flow rate of $5 \mu \mathrm{L} / \mathrm{min}$. Electrospray voltage was set at $\sim 3.5 \mathrm{kV}$ in order to have a stable Taylor cone. The Synapt G2-S was controlled by MassLynx v4.1 SCN 883 (Waters) and operated in positive ion TOF-MS resolution mode, $\mathrm{m} / \mathrm{z}$ range $50-1200 \mathrm{Da}$, scan time $0.1 \mathrm{~s}$, source and interface temperatures were both set at $120^{\circ} \mathrm{C}$, sample cone $20 \mathrm{~V}$, source offset $60 \mathrm{~V}$, automated detector check was off, pDRE lens $99.80 \%$, and detector value was set to an additional $+100 \mathrm{~V}$. In addition, the trap gas was switched from default argon to nitrogen operated at a flow rate of $2 \mathrm{~mL} / \mathrm{min}$, the helium cell DC was set to $0.0 \mathrm{~V}$, and the trap and transfer collision energies were at $1.0 \mathrm{~V}$ to reduce any ion fragmentation in the mass spectrometer. Reaction time $\Delta \mathrm{t}$ was specified as the time difference between the analog signal of the laser pulse and the first mass spectrometric detection event of the reaction product $3 \mathbf{a}$ (Figure 2B). Different reaction tube lengths, 0, 25, 50, 75 and $100 \mathrm{~cm}$ were used to obtain different reaction times. The $y$-axis cut-off in the measurement of reaction time graphs (Figures $2 \mathrm{C}$ and S2) was set to $\Delta \mathrm{t}=0.10 \mathrm{~s}$ in order to allow exponential fitting. In subsequent calculations on reaction time the added value of $\Delta t=0.10 \mathrm{~s}$ was subtracted. Where relevant in figures, the standard deviation of averaged data is provided as error bars. 

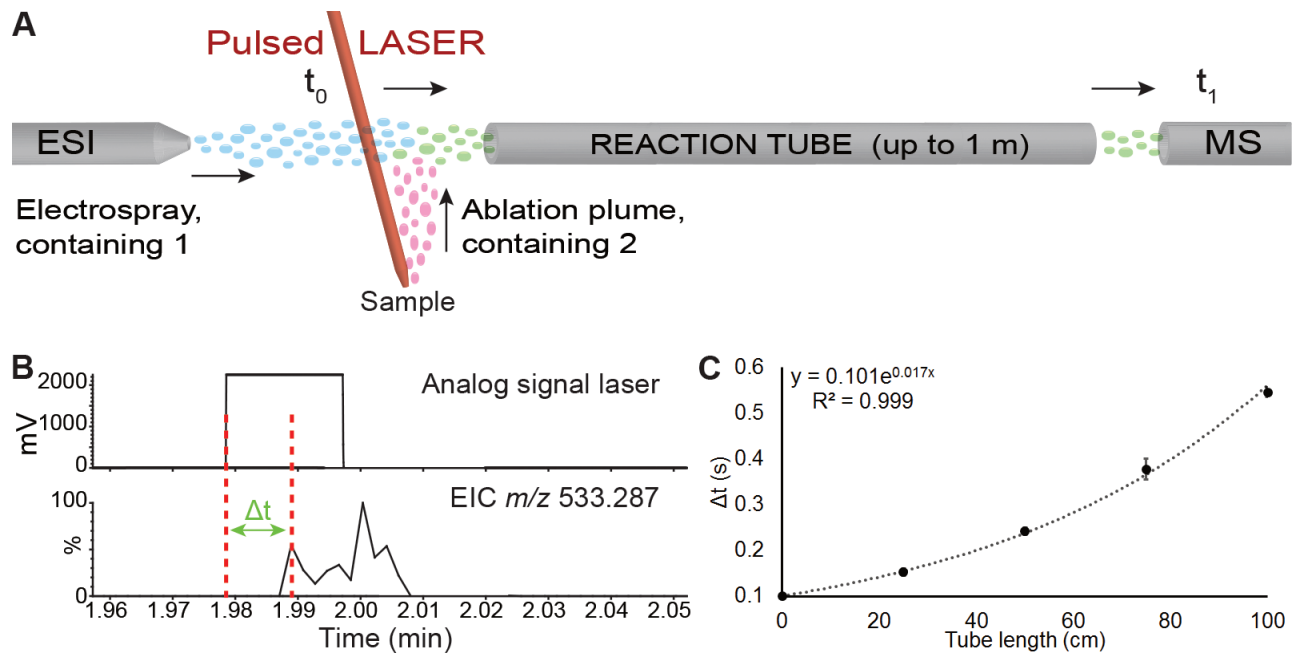

Figure 2. (A) Overview of reactive LAESI setup as used, comprising a pneumatically assisted ESI probe containing click reagent 1, a well plate (not shown) containing click reagent 2, a mid-IR laser and a metal reaction tube $(0-100 \mathrm{~cm})$. Other details can be found in the experimental section and the SI. (B) Specification of reaction time (start time laser pulse time first detection event of product 3a) with (upper trace) the analog signal of the laser pulse and (lower trace) the detection of click reaction product 3a, $m / z$ 533.287. (C) Graph of reaction time versus reaction tube length $(n=2)$.

\section{Determination of Reaction Kinetics}

The experiments that were used to determine the reaction time - described in the previous section - were optimized to achieve maximum temporal resolution, at the cost of signal intensity, with a short MS scan time and few laser pulses per analysis location. The reaction kinetics experiments required larger peak areas for data processing and were optimized for improved signal intensity. For this reason a few acquisition parameters were adjusted from the previous section. The MS scan time was set at $1 \mathrm{~s}$ and 75 laser pulses were used per analysis location to obtain significant reaction product peak areas for reliable integration in the extracted ion currents (EIC) obtained. Reaction time data were now simply calculated for reaction tube lengths of $0,1,2,3,4,5,10,15,20,25,50,75$, and $100 \mathrm{~cm}$, using the exponential fit $\left(y=0.101 \mathrm{e}^{0.017 x}\right)$ of the calibration plot given in Figure $2 \mathrm{C}$. Raw MS data were processed to yield EIC's with a $15 \mathrm{ppm}$ window. These ion currents were mean smoothed (number of smooths was 1, with a window size of 2 scans) and integrated to obtain laser pulse based peak areas. With the kinetic data (as presented in Figure 4, n=3), one outlier at time $19 \mathrm{~ms}$ was removed before averaging with a Grubbs' test based on residuals from the regression line ( $95 \% \mathrm{Cl}, 10 \mathrm{DF})$. Background-subtracted mass spectra were created using the "combine spectrum" function in MassLynx: five scans, each corresponding to 10 laser pulses were averaged, and 50 scans of the electrospray background were subtracted. 


\section{Reactive LAESI Mass Spectrometry Imaging of the Wageningen University (WUR) Logo}

For mass spectrometry imaging the same experimental conditions were used as described in the "Determination of Reaction Kinetics" section, with a few changes. The reaction tube length was fixed at $50 \mathrm{~cm}$, MS inlet and source temperatures were at $150{ }^{\circ} \mathrm{C}$, 20 laser pulses were used per $x-y$ position with a between location dwell time of $0 \mathrm{~s}$, and the LAESI sample stage was cooled further down to $-19{ }^{\circ} \mathrm{C}$ to keep the sample frozen during the experiment. Reactive LAESI data were acquired from a $61 \times 41$ pattern (2501 sample locations) - laser spot size approximately $200 \mu \mathrm{m}$, in-between spot interval $500 \mu \mathrm{m}$ - in a sampling area defined from an optical image, resulting in a total analysis time of $180 \mathrm{~min}$. The sample was created by depositing $200 \mu \mathrm{L}$ of a $1 \mathrm{mM}$ dipyridyl-tetrazine $\mathbf{2 a}$ solution in $\mathrm{H}_{2} \mathrm{O}$ on the hydrophilic part (in the shape of the Wageningen University logo) of an otherwise hydrophobic surface. The sample was then stored in a freezer at $-18^{\circ} \mathrm{C}$ for $30 \mathrm{~min}$ in order to freeze the aqueous solution prior to MSI analysis.

\section{Safety Precautions}

Safety precaution: since the metal reaction tube is in close proximity with the electrospray needle $(\sim 3.5 \mathrm{kV})$, there is risk of electric discharge when not either decently grounded or covered by a nonconductive material. In addition, the open gap between the reaction tube and the MS inlet could contain solvent vapor thus a suction hood is required to remove any harmful solvent vapors that are not sucked into the MS inlet. Finally, there is an open heated MS inlet, which constitutes a potential temperature hazard.

\section{Results and Discussion}

\section{Measurement of Click Reaction Time}

In order to study the reaction time in the click reaction between strained alkyne $\mathbf{1}$ and tetrazine $\mathbf{2 a}$, the following setup was made. A pulsed mid-IR laser $(2.94 \mu \mathrm{m})$ was used to ablate a dipyridyl-tetrazine $\mathbf{2 a}$ solution. The ablation plumes were continuously extracted with orthogonally directed electrospray microdroplets containing $\mathrm{BCN}$-amine 1 reagent. This aerosol is carried along with nebulizer gas through a metal tube before MS detection. To obtain different reaction times, several metal tubes of different lengths $(0,25,50,75$, and 100 $\mathrm{cm}$ ) were used. A schematic overview is presented in Figure $2 \mathrm{~A}$, and an image of the setup is shown Figure $\mathrm{S} 1$. The time difference between the laser pulse and the MS detection of product 3a (Figure $2 \mathrm{~B}$ ) is plotted against increasing tube lengths in Figure $2 \mathrm{C}$. After an initial relatively high linear gas velocity due to the small diameter of nebulizer gas outlet, the velocity is rapidly decreasing, which yields for this range of tube lengths a near-exponential dependence of reaction times on reaction tube length $\left(R^{2}=0.999\right)$. This correlation could therefore be used as a calibration curve for other tube lengths up to $100 \mathrm{~cm}$. The electrical 
voltage offset between the MS inlet and grounded tube was varied to investigate whether charge attraction/repulsion would affect the reaction time; yet, no significant effect was observed (Figure S2).

Figure $3 \mathrm{~A}$ presents a background-subtracted mass spectrum for the reaction between BCN-amine 1 and dipyridyl-tetrazine $\mathbf{2 a}$ after $140 \mathrm{~ms}$ reaction time. Three $\mathrm{m} / \mathrm{z}$ values are observed: protonated dipyridyl-tetrazine $\mathbf{2 a}$ at $\mathrm{m} / \mathrm{z} 237.086$, protonated product $3 \mathbf{a}$ at $\mathrm{m} / \mathrm{z}$ 533.289 and $\mathrm{m} / \mathrm{z}$ 495.148, which corresponds to the sodium adduct of dipyridyl-tetrazine dimer. In order to further demonstrate this methodology, a different click reaction dienophile, TCO-amine 4, was also used as electrospray additive for the reaction with dipyridyl-tetrazine 2a. The obtained background-subtracted mass spectrum is shown in Figure 3B. Like Figure $3 \mathrm{~A}$, both protonated dipyridyl-tetrazine $\mathbf{2 a}$ and its sodium adduct dimer were detected at $\mathrm{m} / \mathrm{z}$ 237.089 and 495.146 , respectively. The reaction product $5 \mathbf{a}$ is detected at $m / z 435.254$, demonstrating the formation of covalently bonded reaction products in reactive LAESI MS. Furthermore, $\mathrm{m} / \mathrm{z}$ value 317.173 was obtained, which is likely to be formed by elimination of the $\mathrm{R}_{2}$ group of structure 5 in Figure 1, the proposed structure is presented in Figure S3 (note: the truncated $R_{2}$ group is already present in $\mathbf{4}$ as an impurity, see Figure S4). Any effect of the nature of the metal from the reaction tube on the reaction was briefly examined by using both copper and stainless steel tubes, and no difference in reaction was observed (Figure S5).
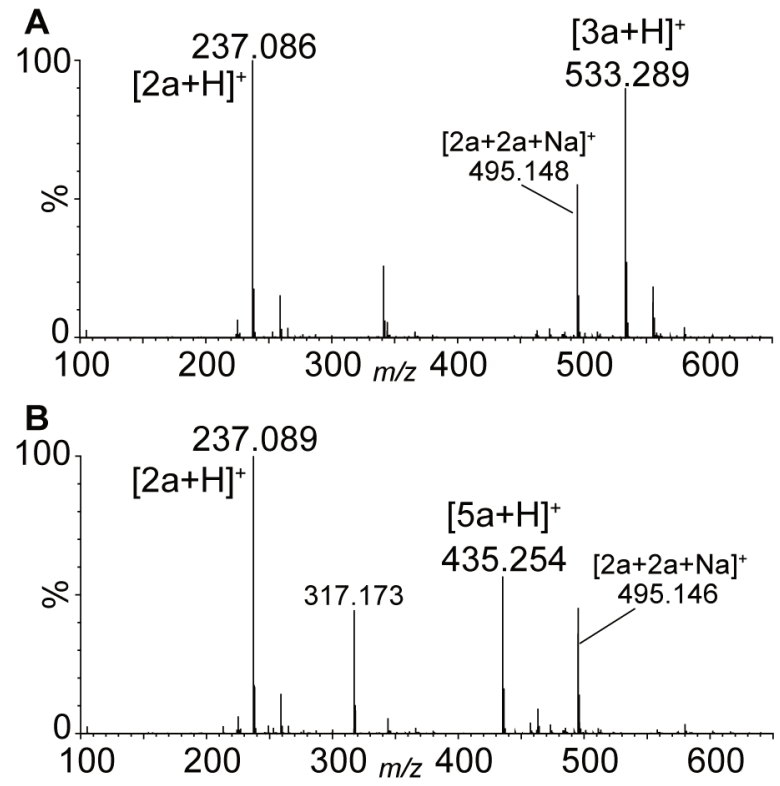

Figure 3. Background-subtracted reactive LAESI mass spectra of the click reaction products between $(A) B C N$-amine 1 and dipyridyl-tetrazine 2a and (B) TCO-amine 4 and dipyridyl-tetrazine 2a, following the setup as depicted in Figure 2 (tube length $=50 \mathrm{~cm}$ ). 
When comparing Figures 3A and S5B a few differences stand out: in Figure 3A both tetrazine $2 \mathrm{a}$ and its dimer are well depicted as a result of the relatively high $(1 \mathrm{mM})$ tetrazine $\mathbf{2 a}$ concentration, whereas in Figure S5B (100 $\mu \mathrm{M}$ tetrazine $\mathbf{2 a}$ concentration) $\mathrm{m} / \mathrm{z}$ value 561.296 is observed; this ion was found to be an adduct between both click reagents (Figure S6), likely due to $\mathrm{H}$-bonding between the protonated amine on $\mathbf{1}$ and the pyridine ring on $\mathbf{2 a}$. $\mathrm{m} / \mathrm{z} 561.296$ is absent in Figure $3 \mathrm{~A}$ due to the higher MS source temperature used $\left(150{ }^{\circ} \mathrm{C}\right.$ instead of $120^{\circ} \mathrm{C}$ ), effectively breaking the supposed $\mathrm{H}$-bond prior to mass analysis.

Finally, the solvent droplet lifetime over the $0.5 \mathrm{~s}(100 \mathrm{~cm})$ experimental timeframe was indirectly investigated by following the area ratio between reaction product $3 \mathbf{a}$ and its $\left[3 \mathbf{a}+\mathrm{H}_{2} \mathrm{O}\right]$ adduct ions. Tabulating these ratio's for multiple tube lengths (Table S7) results in a steady ratio increase corresponding with increasing tube-length. This data suggests gradual droplet evaporation inside our reaction tube, but the microdroplets do not seem to completely evaporate even at distances up to $100 \mathrm{~cm}$, as the $\mathrm{H}_{2} \mathrm{O}$ adduct is still detected here (Figure S8). This is in accordance with earlier reported results by Zare and co-workers, ${ }^{43}$ where the evaporation of water microdroplets produced by an electrospray probe was found to be negligible, although in a much shorter timeframe of $50 \mu \mathrm{s}$.

\section{Determination of Model Click Reaction System Kinetics}

Following the time measurements and detection of click reaction products $\mathbf{3} \mathbf{a}$ and $\mathbf{5 a}$, which are both only possible due to the insertion of a reaction tube, the TRMS method was used to study the IEDDA reaction kinetics in charged microdroplets under pseudo-first order conditions. The latter was achieved with an excess of BCN-amine 1, as the concentration in the electrospray microdroplets is much larger than the dipyridyl-tetrazine $\mathbf{2 a}$ in the ablated liquid solution (Figure S9). Reserpine was added as internal standard to the dipyridyl-tetrazine $\mathbf{2 a}$ solution in order to correct for possible ablation variation and any bias caused by changing the distance between the electrospray source and MS inlet via stepwise variation of the reaction tube length (13 different tube lengths were used). The product $3 \mathbf{a}$ formation over time is presented in Figure 4, from which the pseudo-first order rate constant for the IEDDA reaction was determined to be $8 \mathrm{~s}^{-1}$. One additional interesting finding was that the absolute peak areas of both click reaction product $\mathbf{3 a}$ and the used standard reserpine were surprisingly stable over reaction tube lengths longer than $15 \mathrm{~cm}$, indicating that almost no signal was lost upon increasing the reaction tube length even up to $1 \mathrm{~m}$, corresponding to $0.5 \mathrm{~s}$ reaction time (Figure S10).

The second-order rate constant $k_{2}$ is then derived as $8 \mathrm{~s}^{-1} / 0.10 \mathrm{mM}=8 \times 10^{4}$ $\mathrm{M}^{-1} \mathrm{~s}^{-1}$. The analogous reaction between dipyridyl-tetrazine $\mathbf{2 a}$ and $\mathrm{BCN}-\mathrm{OH}$ performed in methanol solution was reported in various studies to have a $k_{2}$ of typically $10^{2} \mathrm{M}^{-1} \mathrm{~s}^{-1} \cdot{ }^{74,75}$ The current reaction in charged microdroplets is at least two orders of magnitude faster than the similar click reaction in bulk solvents. It has been reported that the addition of water as cosolvent $\left(55: 45 \mathrm{MeOH}: \mathrm{H}_{2} \mathrm{O}\right)$ increases the rate constant due to hydrogen bonding with 
tetrazine, which reduces the highest occupied molecular orbital - lowest unoccupied molecular orbital (HOMO-LUMO) gap. ${ }^{76}$ The effect of a decreasing $\mathrm{pH}$ could also contribute to the increased reaction rate because of possible protonation of the pyridine ring, thus increasing its electron-withdrawing properties and concomitantly speeding up the reaction. Finally, evaporation of solvent molecules could also cause the reaction rate to increase as a result of increasing concentration, however, solvent droplets do not seem to fully evaporate before entering the vacuum of the MS inlet, as discussed above. It is therefore likely that the observed reaction acceleration is due to a combination of effects originating from the charged microdroplet environment, as discussed by Cooks and co-workers. ${ }^{79}$

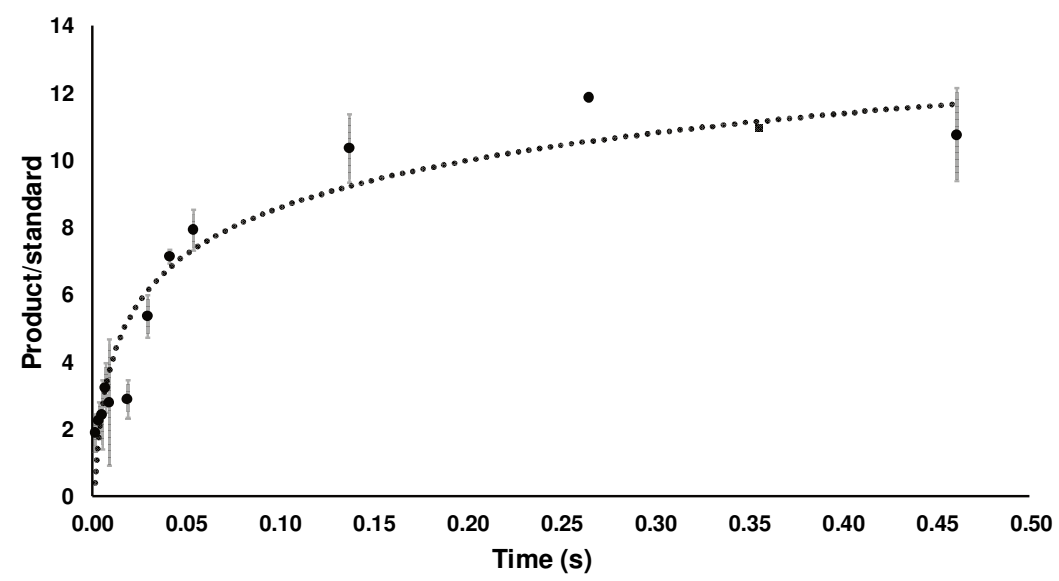

Figure 4. Graph of the formation of click reaction product $3 a$ in relation to reaction time $(n=3)$ following insertion of 13 different reaction tube lengths $(0$ to $100 \mathrm{~cm}$ ) for the model reaction between $\mathrm{BCN}$-amine $\mathbf{1}$ and dipyridyl-tetrazine $\mathbf{2 a}$.

\section{Reactive LAESI Robustness and Scope}

The robustness of the methodology and its application to various tetrazines with different polarities was briefly investigated using clofentezine $\mathbf{2 b}$ and diphenyl-tetrazine $\mathbf{2 c}$. For this, four rows of a 96-well plate were filled with different tetrazine solutions per row, from row $A$ to $D$; blank, dipyridyl-tetrazine $\mathbf{2 a}$, clofentezine $\mathbf{2 b}$, and diphenyl-tetrazine $\mathbf{2 c}$, all $1 \mathrm{mM}$ in $\mathrm{MeOH}-\mathrm{H}_{2} \mathrm{O}$ (95:5). Subsequently, the analysis was run starting at row $\mathrm{A}$ and followed a typewriter pattern to end in row D. Unprocessed EIC's and the analog signal of the laserpulsed injection of tetrazines are shown in Figure 5. 


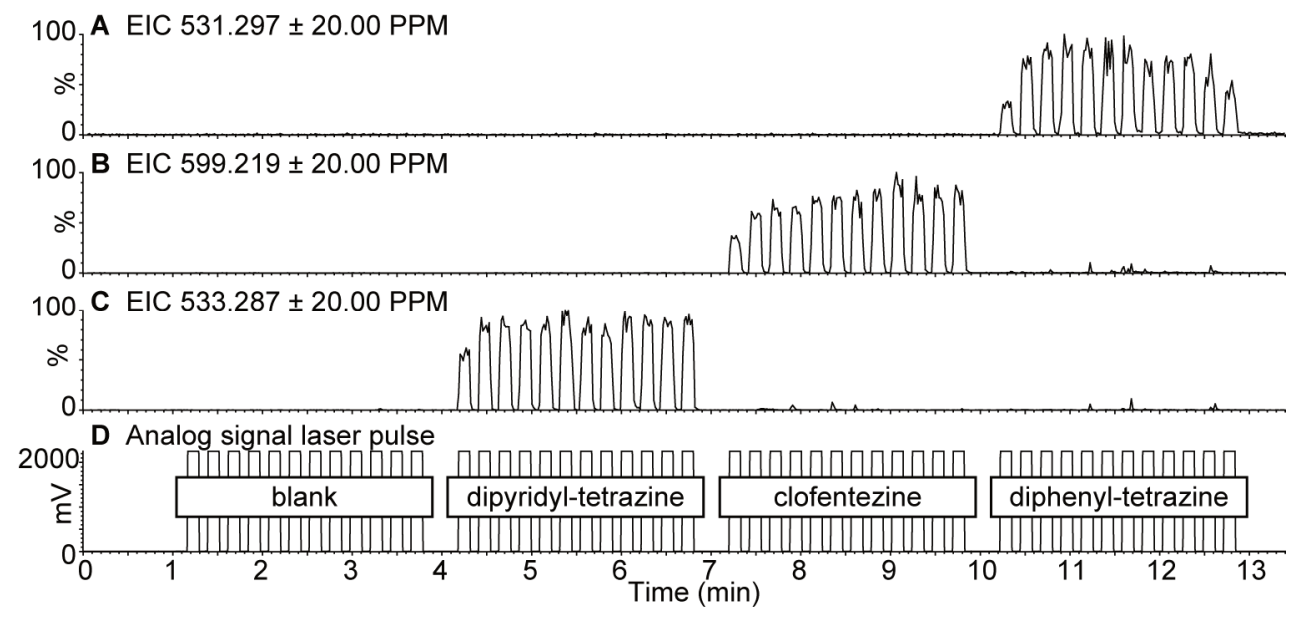

Figure 5. Reactive LAESI MS extracted ion currents of the click reaction products of 1 with (A) diphenyl-tetrazine $3 \mathrm{c}$ ( $m / z$ 531.297), (B) clofentezine 3b ( $\mathrm{m} / \mathbf{z}$ 599.219), and (C) dipyridyl-tetrazine 3a $(\mathrm{m} / \mathrm{z} 533.287)$. Corresponding background-subtracted mass spectra are given in Figures S11A, S11B and 3A, respectively. In addition, the analog signal of the laser pulses is displayed (D). The reaction tube length was $50 \mathrm{~cm}$.

In the blank, at 1 to 4 min, no signal was obtained in the EIC of any BCN-amine 1 tetrazine products. The click reaction product ions of diphenyl-tetrazine $3 c(5 A)$, clofentezine 3b $(5 \mathrm{~B})$, and dipyridyl-tetrazine $3 \mathbf{a}(5 \mathrm{C})$ were in excellent agreement with the timeframe of the analog signal of ablation from the corresponding tetrazine. Although not studied, other (asymmetrical) tetrazines are expected to work as well. The EIC's in Figure 5 also demonstrate that well-to-well signal intensity stability is at least equal to or even better than that of conventional LAESI experiments (Figure S12), showing that ambient mass spectrometry imaging with reactive LAESI would be realistic.

Thanks to reactive LAESI, the sensitivity could be increased because of a higher ionization efficiency of the click reaction product $\mathbf{3 a}$ in comparison with dipyridyl-tetrazine $\mathbf{2 a}$. This effect of increased sensitivity combined with indications of the scope of reactive LAESI are shown in Figure 6, for which dipyridyl-tetrazine 2a was dissolved in both $\mathrm{H}_{2} \mathrm{O}$ and in ten times diluted soft drink (cola) at a concentration of $100 \mu \mathrm{M}$ and analyzed with (Figure 6A) and without (Figure $6 \mathrm{~B}$ ) $\mathrm{BCN}$-amine 1 in the ESI spray solution. As a result, the click reaction product $3 \mathbf{a}$ could still be detected in diluted cola, whereas unreacted dipyridyl-tetrazine $\mathbf{2 a}$ got lost in the background. 


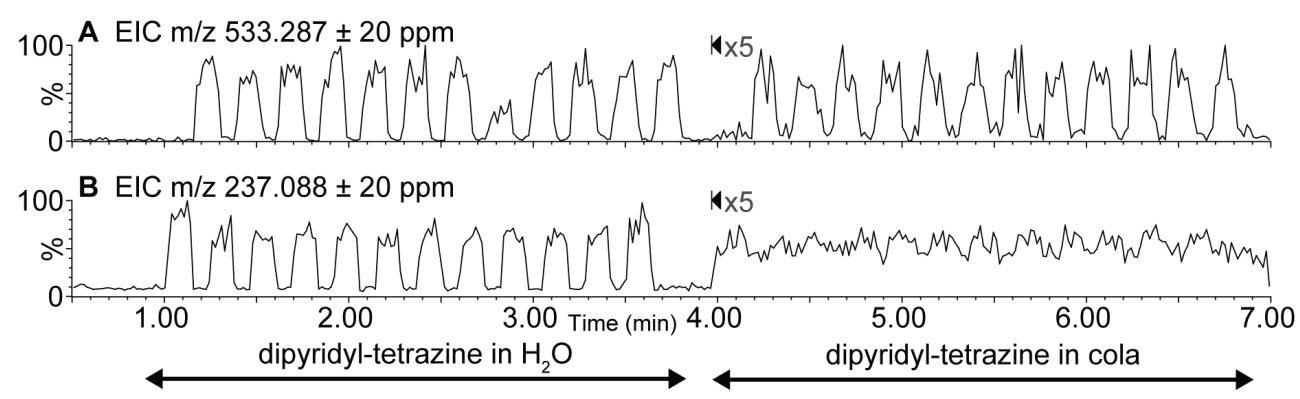

Figure 6. Reactive LAESI extracted ion currents of two separate analyses of a $100 \mu \mathrm{M}$ dipyridyl-tetrazine $2 \mathrm{a}$ solution in $\mathrm{H}_{2} \mathrm{O}$ and in ten times diluted cola. Analysis (A) was with $\mathrm{BCN}$-amine $\mathbf{1}$ in the electrospray solution and depicts the pyridazine product $\mathbf{3 a}(\mathrm{m} / \mathrm{z} 533.287)$ at all times. Analysis $(\mathrm{B})$ was without $\mathrm{BCN}$-amine $\mathbf{1}$ in the electrospray solution and shows dipyridyl-tetrazine $\mathbf{2 a}(\mathrm{m} / \mathrm{z} 237.088)$ in a water environment but not in cola.

\section{Reactive LAESI Mass Spectrometry Imaging}

Finally, preliminary reactive LAESI mass spectrometry imaging (MSI) experiments were conducted on a dipyridyl-tetrazine 2 a solution which was deposited - and subsequently frozen - onto a hydrophilic surface area in the shape of the Wageningen University logo (Figure S13; size $20 \mathrm{~mm} \times 29 \mathrm{~mm}$ ). An image was created by plotting the absolute intensity of the protonated click reaction product $3 a$ according to laser ablation $x-y$ coordinates. The resulting $2 \mathrm{D}$ ion map nicely correlates with the sample (Figure 7 ), showing the potential to map the presence of compounds onto a surface using reactive LAESI MSI.

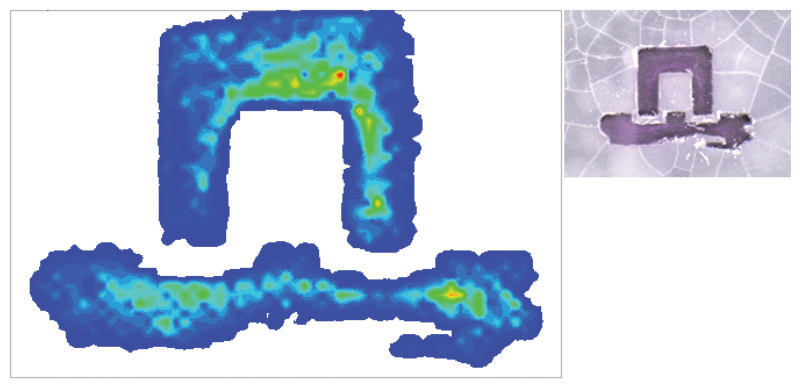

Figure 7. Reactive LAESI-MSI ion map of the Wageningen University logo via $500 \mu \mathrm{m}$ spatial resolution - see experimental section - of $\mathrm{m} / \mathrm{z} 533.287 \pm 0.01 \mathrm{Da}$, being the click reaction product $3 \mathrm{a}[\mathrm{M}+\mathrm{H}]^{+}$. 


\section{Conclusion}

In conclusion, we have developed a novel ambient ionization time-resolved mass spectrometry method for performing and studying reactions in charged microdroplets. For this we developed a setup to allow the first reactive LAESI analysis with covalent bond-forming (click) reactions. The observation of the click reaction products was a result of the substantially increased reaction time due to the insertion of a reaction tube. Following a calibration of the exponential relationship between reaction time and reaction tube length using the time difference between the laser pulse and MS detection signals, we were able to determine kinetic data for the model system IEDDA (click) reaction between a tetrazine with a strained cycloalkyne. Additionally, this method has shown excellent stability and improved sensitivity, and proved to be viable for several click reactions. With this reactive LAESI method, a wide range of molecular reactions can potentially be studied, to investigate rates, solvent effects on molecular reactions, or as a tool to improve the detection of target analytes in ambient ionization MS(I). Following these encouraging results, this reactive LAESI method could also be extended to other reactions previously reported in reactive DESI, like in the analyses of, e.g., carbohydrates with boronic acids, ${ }^{62}$ steroids with hydroxylamine,${ }^{31}$ and/or protein - ligand interactions. ${ }^{80}$ Eventually, the present work is expected to enable in-situ reactions during bioanalytical MSI.

\section{Acknowledgements}

The authors thank Frank Claassen and Jan Commandeur for technical assistance, Sevil Sahin for the NMR measurement and Martine Keijzer for preliminary experiments. The authors are grateful to receive funding for this research from The Netherlands Organization for Scientific Research (NWO) in the framework of the Technology Area TA-COAST2 of the Fund New Chemical Innovations (Project No. 053.21.111). 


\section{References}

Biemann, K. Angew. Chem. Int. Ed. 1962, 1, 98-111.

Petras, D.; Jarmusch, A. K.; Dorrestein, P. C. Curr. Opin. Chem. Biol. 2017, 36, 24-31.

Aebersold, R.; Mann, M. Nature 2016, 537, 347.

Ingram, A. J.; Boeser, C. L.; Zare, R. N. Chem. Sci. 2016, 7, 39-55.

Lee, E. D.; Mueck, W.; Henion, J. D.; Covey, T. R. J. Am. Chem. Soc. 1989, 111, 4600-4604.

Rob, T.; Wilson, D. J. J. Am. Soc. Mass Spectrom. 2009, 20, 124-130.

Pan, J.; Rintala-Dempsey, A. C.; Li, Y., et al. Biochemistry 2006, 45, 3005-3013. Liuni, P.; Olkhov-Mitsel, E.; Orellana, A.; Wilson, D. J. Anal. Chem. 2013, 85, 3758-3764.

Mortensen, D. N.; Williams, E. R. J. Am. Chem. Soc. 2016, 138, 3453-3460.

Jacobs, M. I.; Davies, J. F.; Lee, L., et al. Anal. Chem. 2017, 89, 12511-12519.

Zhu, L.; Gamez, G.; Chen, H. W., et al. Rapid Commun. Mass Spectrom. 2008, 22, 2993-2998.

Gamez, G.; Zhu, L.; Disko, A., et al. Chem. Commun. 2011, 47, 4884-4886.

Lento, C.; Wilson, D. J. Analyst 2017, 142, 1640-1653.

Banerjee, S.; Zare, R. N. Angew. Chem. Int. Ed. 2015, 127, 15008-15012.

Girod, M.; Moyano, E.; Campbell, D. I.; Cooks, R. G. Chem. Sci. 2011, 2, 501-510.

Perry, R. H.; Splendore, M.; Chien, A., et al. Angew. Chem. Int. Ed. 2011, 50, 250254.

Bain, R. M.; Pulliam, C. J.; Thery, F.; Cooks, R. G. Angew. Chem. Int. Ed. 2016, $128,10634-10638$.

Loren, B. P.; Wleklinski, M.; Koswara, A., et al. Chem. Sci. 2017, 8, 4363-4370.

Yan, X.; Cheng, H.; Zare, R. N. Angew. Chem. Int. Ed. 2017, 56, 3562-3565.

Badu-Tawiah, A. K.; Campbell, D. I.; Cooks, R. G. J. Am. Soc. Mass Spectrom. 2012, 23, 1461-1468.

Wleklinski, M.; Falcone, C. E.; Loren, B. P., et al. Eur. J. Org. Chem. 2016, 54805484.

Müller, T.; Badu-Tawiah, A.; Cooks, R. G. Angew. Chem. Int. Ed. 2012, 51, 1183211835.

Cheng, S.; Wu, Q.; Xiao, H.; Chen, H. Anal. Chem. 2017, 89, 2338-2344.

Waldner, M. J.; Neurath, M. F. Curr. Gene Ther. 2009, 9, 239-247.

Mann, S. Angew. Chem. Int. Ed. 2013, 52, 155-162.

Michaud, V.; El Haddad, I.; Yao, L., et al. Atmos. Chem. Phys. 2009, 9, 51195130.

Chen, H.; Cotte-Rodriguez, I.; Cooks, R. G. Chem. Commun. 2006, 597-599.

Wu, C.; Qian, K.; Nefliu, M.; Cooks, R. G. J. Am. Soc. Mass Spectrom. 2010, 21, 261-267.

Rao, W.; Mitchell, D.; Licence, P.; Barrett, D. A. Rapid Commun. Mass Spectrom. 2014, 28, 616-624.

Nyadong, L.; Green, M. D.; de Jesus, V. R., et al. Anal. Chem. 2007, 79, 21502157.

Huang, G.; Chen, H.; Zhang, X., et al. Anal. Chem. 2007, 79, 8327-8332.

Cotte-Rodríguez, I.; Takáts, Z.; Talaty, N., et al. Anal. Chem. 2005, 77, 67556764.

Polenz, I.; Brosseau, Q.; Baret, J.-C. Soft Matter 2015, 11, 2916-2923.

Gruner, P.; Riechers, B.; Semin, B., et al. Nat. Commun. 2016, 7, 10392.

Rideout, D. C.; Breslow, R. J. Am. Chem. Soc. 1980, 102, 7816-7817.

Narayan, S.; Muldoon, J.; Finn, M. G., et al. Angew. Chem. Int. Ed. 2005, 44, 3275-3279. 
Li, Y.; Yan, X.; Cooks, R. G. Angew. Chem. Int. Ed. 2016, 128, 3494-3498.

Lee, J. K.; Banerjee, S.; Nam, H. G.; Zare, R. N. Q. Rev. Biophys. 2015, 48, 437444.

Bain, R. M.; Pulliam, C. J.; Cooks, R. G. Chem. Sci. 2015, 6, 397-401.

Takáts, Z.; Wiseman, J. M.; Gologan, B.; Cooks, R. G. Science 2004, 306, 471473.

Chen, H.; Venter, A.; Cooks, R. G. Chem. Commun. 2006, 2042-2044.

Venter, A.; Sojka, P. E.; Cooks, R. G. Anal. Chem. 2006, 78, 8549.

Lee, J. K.; Kim, S.; Nam, H. G.; Zare, R. N. Proc. Natl. Acad. Sci. U.S.A 2015, 112, 3898-3903.

Nemes, P.; Huang, H.; Vertes, A. Phys. Chem. Chem. Phys. 2012, 14, 2501-2507. Nazari, M.; Ekelöf, M.; Khodjaniyazova, S., et al. Rapid Commun. Mass Spectrom. 2017, 31, 1868-1874.

van Geenen, F. A. M. G.; Franssen, M. C. R.; Schotman, A. H. M., et al. Anal. Chem. 2017, 89, 4031-4037.

Nemes, P.; Barton, A. A.; Li, Y.; Vertes, A. Anal. Chem. 2008, 80, 4575-4582.

Vertes, A.; Nemes, P.; Shrestha, B., et al. Appl. Phys. A 2008, 93, 885-891.

Sripadi, P.; Nazarian, J.; Hathout, Y., et al. Metabolomics 2009, 5, 263-276.

Nemes, P.; Barton, A. A.; Vertes, A. Anal. Chem. 2009, 81, 6668-6675.

Nemes, P.; Vertes, A. In Methods Mol. Biol., 2010, 159-171.

Shrestha, B.; Vertes, A. Anal. Chem. 2009, 81, 8265-8271.

Shrestha, B.; Vertes, A. J. Vis. Exp. 2010, e2144.

Shrestha, B.; Patt, J. M.; Vertes, A. Anal. Chem. 2011, 83, 2947-2955.

Nemes, P.; Vertes, A. Anal. Chem. 2007, 79, 8098-8106.

Liu, Y.; Miao, Z.; Lakshmanan, R., et al. Int. J. Mass Spectrom. 2012, 325-327, 161-166.

Barbara, J. E.; Eyler, J. R.; Powell, D. H. Rapid Commun. Mass Spectrom. 2008, 22, 4121-4128.

Lebeau, D.; Reiller, P. E.; Lamouroux, C. Talanta 2015, 132, 877-883.

Lostun, D.; Perez, C. J.; Licence, P., et al. Anal. Chem. 2015, 87, 3286-3293.

Shin, E.; Cha, S. Bull. Korean Chem. Soc. 2018, 39, 40-44.

Nefliu, M.; Cooks, R. G.; Moore, C. J. Am. Soc. Mass Spectrom. 2006, 17, 10911095.

Zhang, Y.; Chen, H. Int. J. Mass Spectrom. 2010, 289, 98-107.

Wu, C.; Ifa, D. R.; Manicke, N. E.; Cooks, R. G. Anal. Chem. 2009, 81, 7618-7624.

Song, Y.; Cooks, R. G. J. Mass Spectrom. 2007, 42, 1086-1092.

Tian, Y.; Chen, J.; Ouyang, Y., et al. Anal. Chim. Acta 2014, 814, 49-54.

Ding, J.; Gu, H.; Yang, S., et al. Anal. Chem. 2009, 81, 8632-8638.

Jiang, J.; Chen, S.; Li, M., et al. Anal. Lett. 2017, 50, 797-805.

Tang, F.; Guo, C.; Ma, X., et al. Anal. Chem. 2018, 90, 5612-5619.

Devenport, N. A.; Blenkhorn, D. J.; Weston, D. J., et al. Anal. Chem. 2014, 86, 357-361.

Shrestha, B.; Nemes, P.; Nazarian, J., et al. Analyst 2010, 135, 751-758.

Nazari, M.; Malico, A. A.; Ekelöf, M., et al. Anal. Bioanal. Chem. 2018, 410, 953962.

Elliott, T. S.; Bianco, A.; Townsley, F. M., et al. Cell Chem. Biol. 2016, 23, 805815.

Erdmann, R. S.; Takakura, H.; Thompson, A. D., et al. Angew. Chem. Int. Ed. 2014, 53, 10242-10246.

Chen, W.; Wang, D.; Dai, C., et al. Chem. Comm. 2012, 48, 1736-1738.

Wang, D.; Chen, W.; Zheng, Y., et al. Org. Biomol. Chem. 2014, 12, 3950-3955.

Lang, K.; Davis, L.; Wallace, S., et al. J. Am. Chem. Soc. 2012, 134, 10317-10320.

Bruins, J. J.; Albada, B.; van Delft, F. Chem. Eur. J 2018, 24, 4749-4756. 
Reactive LAESI Time-Resolved Mass Spectrometry of Click Reactions

78 Goor, O. J. G. M.; Brouns, J. E. P.; Dankers, P. Y. W. Polym. Chem. 2017, 8, 52285238.

79 Yan, X.; Bain, R. M.; Cooks, R. G. Angew. Chem. Int. Ed. 2016, 55, 12960-12972.

80 Liu, P.; Zhang, J.; Ferguson, C. N., et al. Anal. Chem. 2013, 85, 11966-11972. 


\section{Supporting Information Chapter 3}

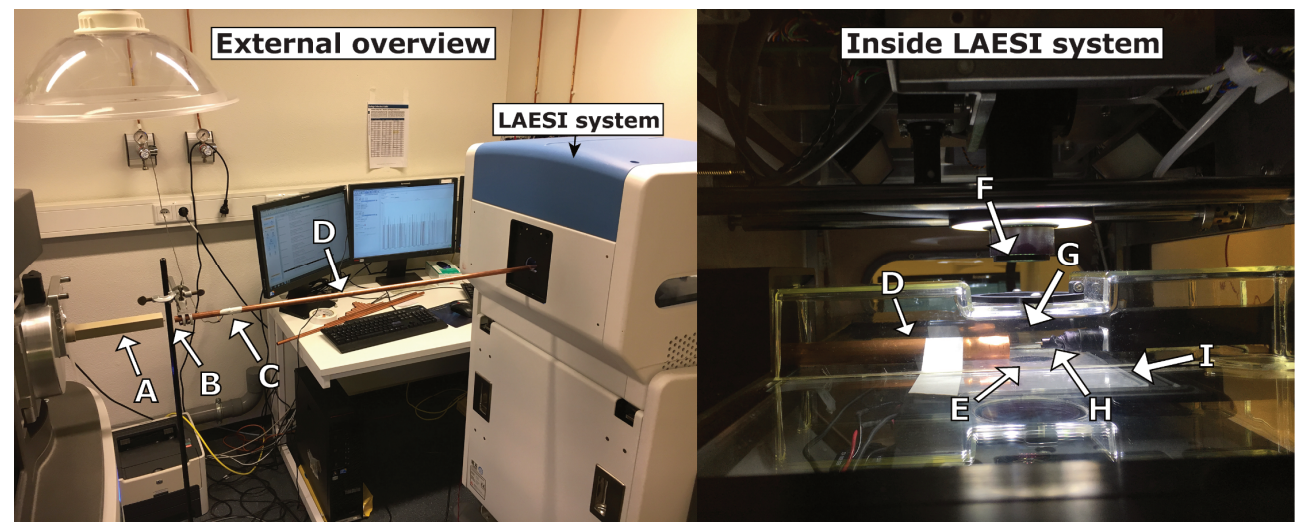

Figure S1. Overview of reactive LAESI instrumental setup. (A) Heated MS inlet, (B) $20 \mathrm{~mm}$ distance between MS inlet and metal reaction tube, $(C)$ wire to ground metal tube, $(D)$ metal reaction tube (lengths 0 to $100 \mathrm{~cm}$ ), (E) sample cover plate (hole $\varnothing 2 \mathrm{~cm}$ ), (F) mid-IR laser focussing lens, (G) $18 \mathrm{~mm}$ distance between ESI spray and metal tube, $(\mathrm{H}) \mathrm{ESI}$ spray needle with nebulizer, and (I) 96 well plate.

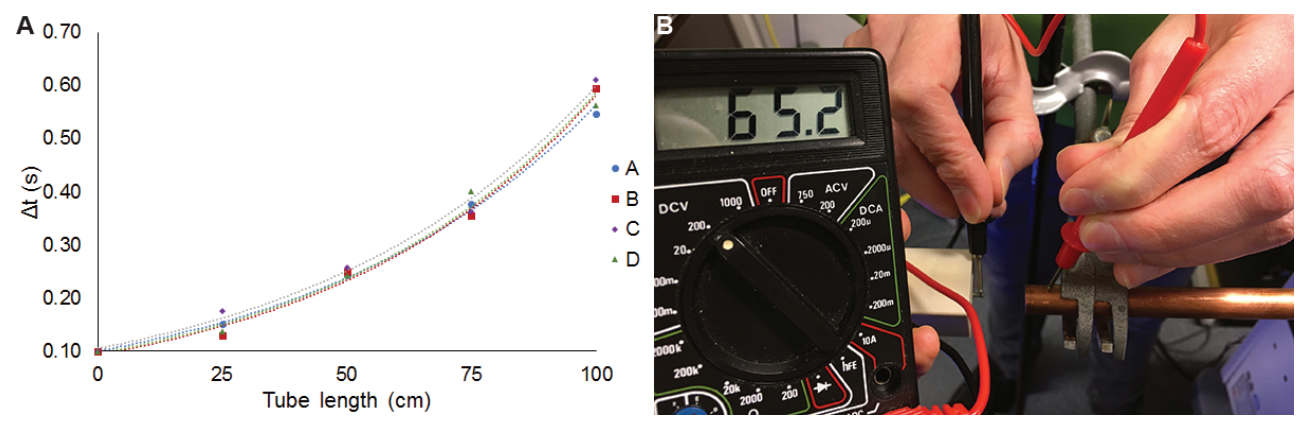

Figure S2. Graph of reaction time vs tube length. (A) Reaction time is plotted against used tube lengths with different ESI and counter electrode (MS inlet) voltage settings: [A] $3500 \mathrm{~V}$ ESI and $65.2 \mathrm{~V}$ MS inlet to grounded tube; [B] $2500 \mathrm{~V}$ ESI and 65.2 V MS inlet to grounded tube; [C] $3500 \mathrm{~V} \mathrm{ESI} \mathrm{and} \mathrm{-23.0} \mathrm{V} \mathrm{MS} \mathrm{inlet} \mathrm{to} \mathrm{grounded} \mathrm{tube;} \mathrm{[D]} 3500 \mathrm{~V}$ ESI and $0.1 \mathrm{~V}$ MS inlet to grounded tube. Apparently, generated microdroplets are propelled by nebulizing gas through the reaction tube and subsequently drawn by vacuum into the MS, without being influenced by offset voltage settings. (B) Optical image of voltage measurement between grounded metal tube and MS inlet (counter electrode). 


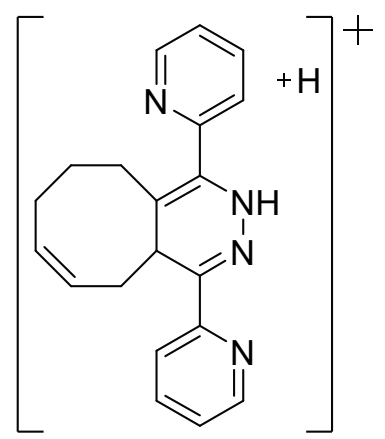

Figure S3. Proposed structure of $m / z 317.173$.

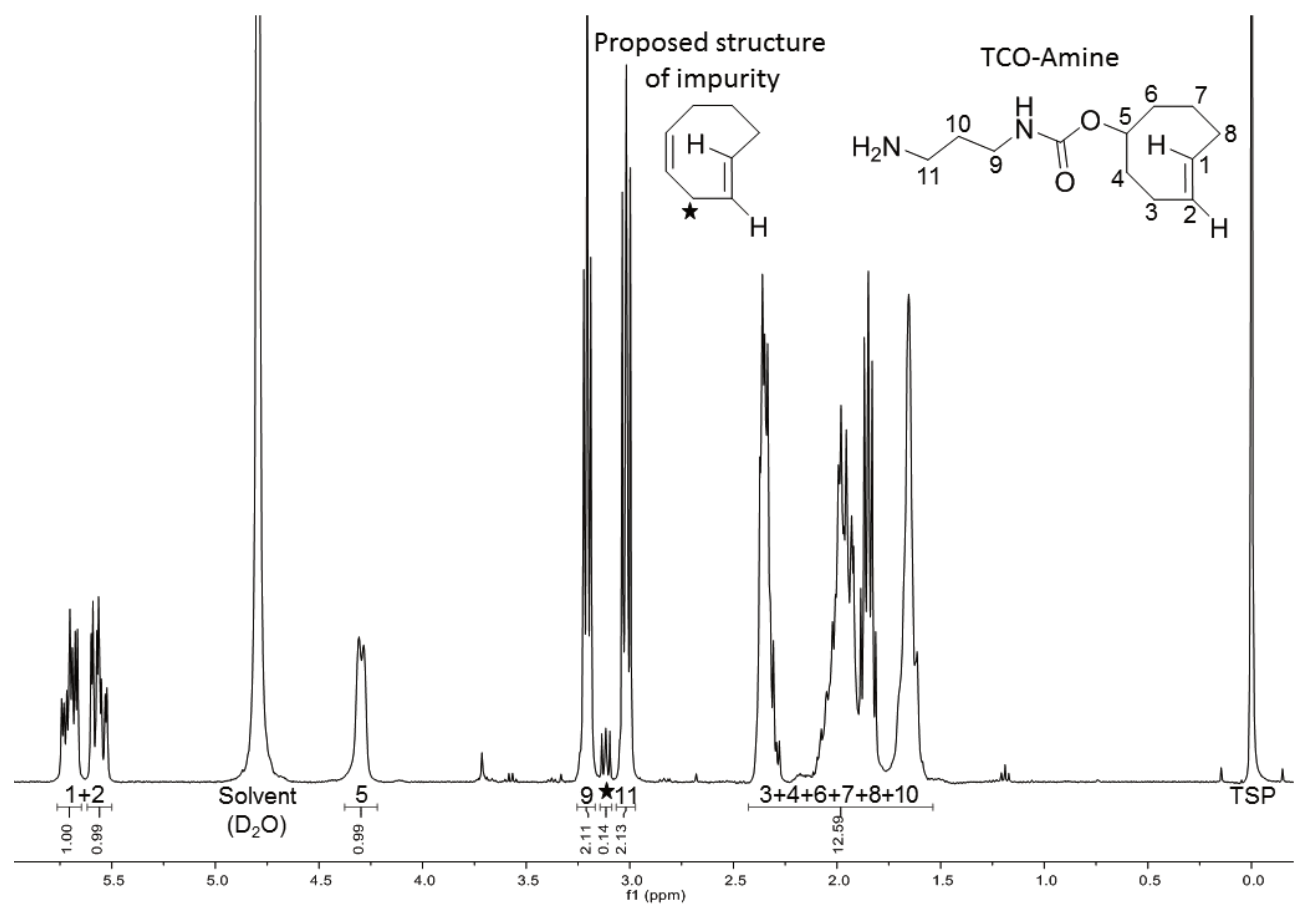

Figure S4. ${ }^{1} \mathrm{H}-\mathrm{NMR}$ spectrum of structure 4. An impurity was detected - the triplet is marked with a star - in the ${ }^{1} \mathrm{H}-\mathrm{NMR}$ spectrum of structure 4 in $\mathrm{D}_{2} \mathrm{O}$. The proposed structure of the impurity would lead to MS detection of proposed product in Figure $\mathrm{S} 3$ at $m / z 317.173$. 

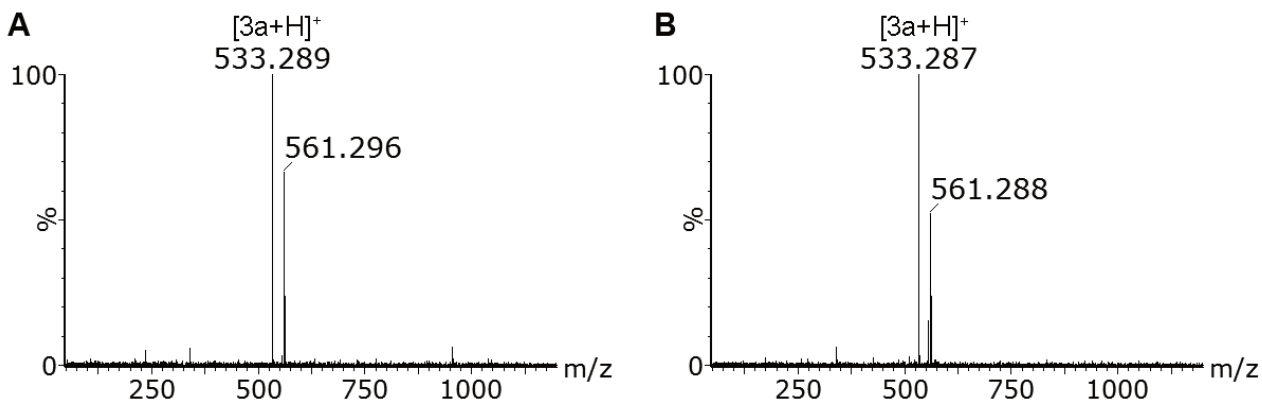

Figure S5. Background-subtracted mass spectra of the IEDDA reaction between BCN-amine 1 and dipyridyl-tetrazine 2a with (A) stainless steel and (B) copper tubes. Tube lengths of $100 \mathrm{~cm}$ were used for both experiments. $m / z 561.29$ is likely to be an adduct between the highly functionalized BCN-amine $\mathbf{1}$ and dipyridyl-tetrazine $\mathbf{2 a}$. The absence of a BCNamine $1 \mathrm{~m} / \mathrm{z}$ value in the spectra is because it was part of the ESI spray solution and thus background subtracted.

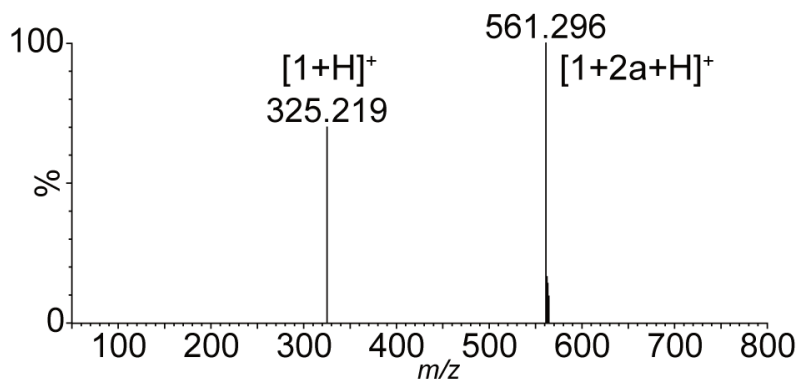

Figure S6. $\mathrm{MS}^{2}$ on $\mathrm{m} / \mathrm{z}$ 561.3. Protonated $\mathrm{BCN}$-amine 1 is obtained following MS/MS on the ion of $\mathrm{m} / \mathrm{z} 561.3$ indicating adduct formation between both $\mathrm{BCN}$-amine 1 and dipyridyl-tetrazine $2 \mathrm{a}$ reagents.

\begin{tabular}{|c|c|}
\hline Tube length $(\mathrm{cm})$ & {$[3 \mathrm{a}+\mathrm{H}]^{+} /\left[3 \mathrm{a}+\mathrm{H}_{3} \mathrm{O}\right]^{+}$} \\
\hline 2 & 0.4 \\
\hline 5 & 0.5 \\
\hline 10 & 0.8 \\
\hline 25 & 0.9 \\
\hline 50 & 1.2 \\
\hline 75 & 1.5 \\
\hline 100 & 2.0 \\
\hline
\end{tabular}

Table S7. Table of tube length with corresponding area ratio of protonated product $\mathbf{3 a}$ and its water adduct. 


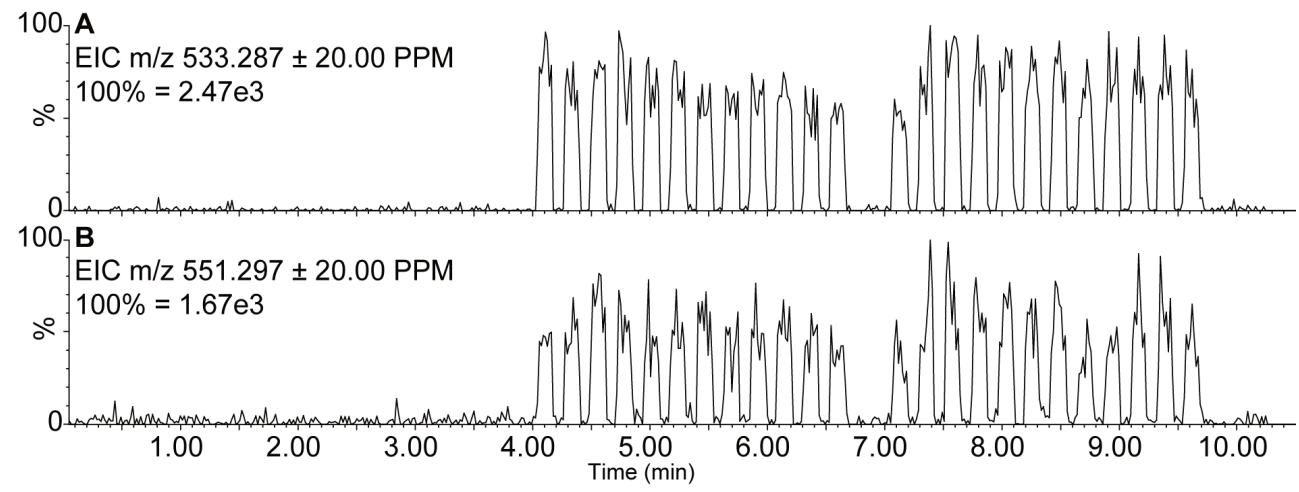

Figure S8. Reactive LAESI EIC's of product 3a and its water adduct. (A) EIC of $m / z 533.287 \pm 20$ ppm (click reaction product, 3a) and (B) EIC of $m / z 551.297 \pm 20$ ppm $\left(\mathrm{H}_{2} \mathrm{O}\right.$ adduct of product $\left.3 \mathbf{a}\right)$ in the same experiment. Reaction tube $100 \mathrm{~cm}$.

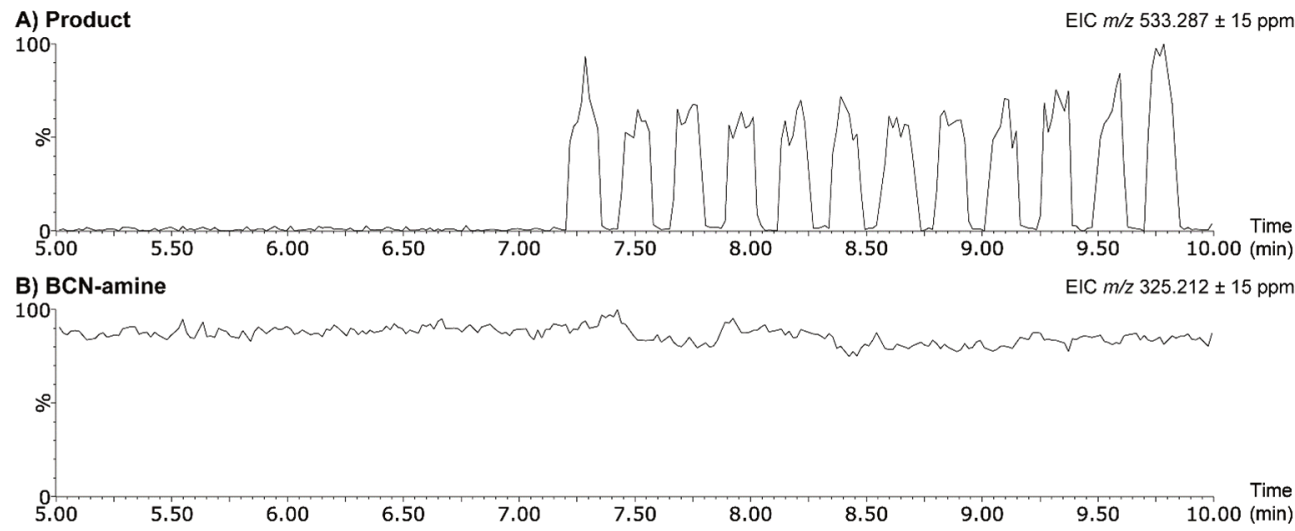

Figure S9. Reactive LAESI EIC's of click reaction product 3a and BCN-amine 1. (A) EIC of $\mathrm{m} / z 533.287 \pm 15 \mathrm{ppm}$ (product 3a) and (B) EIC of $\mathrm{m} / z 325.212 \pm 15 \mathrm{ppm}$ (BCN-amine 1) in the same experiment. No negative peaks in BCNamine 1 signal are observed at the time of product 3 a detection, demonstrating an excess of $\mathrm{BCN}$-amine 1 in the electrospray solution and thus conditions for pseudo-first order kinetics. 

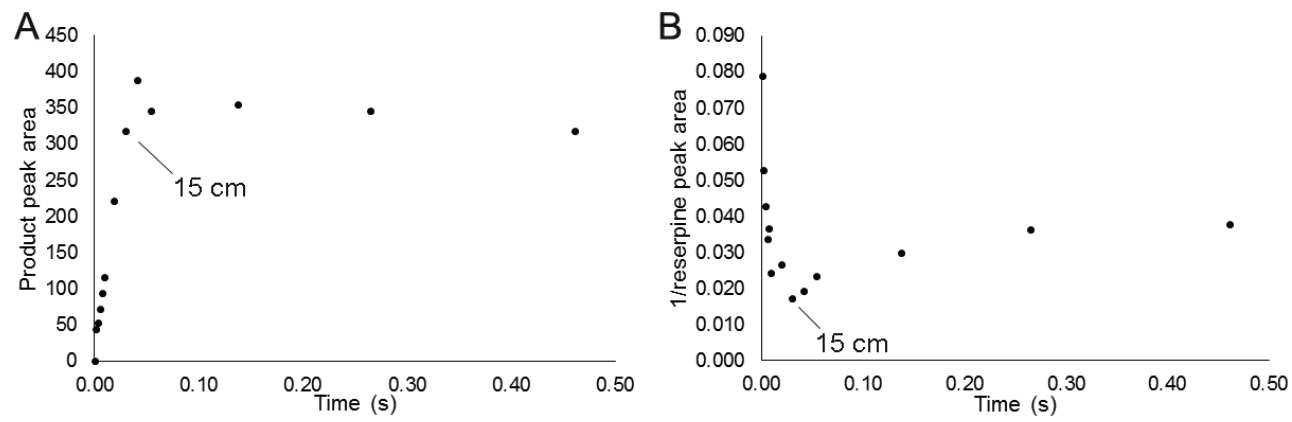

Figure S10. Graphs of absolute peak areas of product 3a and 1/reserpine. (A) Absolute peak area product 3a and (B) 1/absolute peak area reserpine (used as internal standard). Peak areas of both product $\mathbf{3 a}$ and reserpine were only slightly decreasing at reaction tube distances over $15 \mathrm{~cm}(30 \mathrm{~ms})$.

A

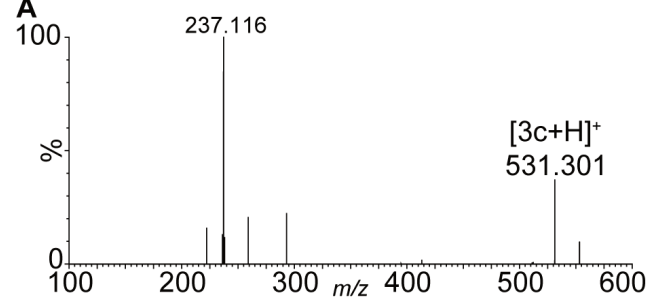

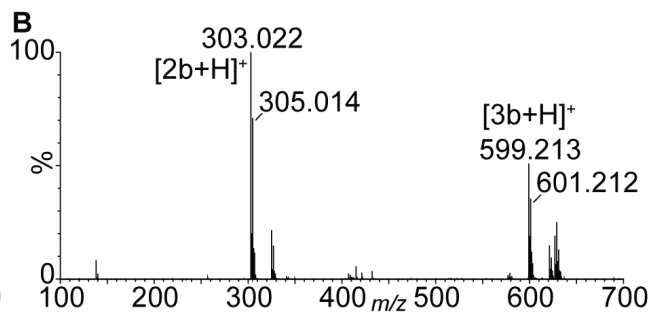

Figure S11. Reactive LAESI background-subtracted mass spectra of the reaction of BCN-amine 1 with diphenyltetrazine $\mathbf{2 c}$ and clofentezine $\mathbf{2 b}$. (A) Background subtracted mass spectrum for the reaction of diphenyl-tetrazine (2c) to give 3c. $m / z 237.116$ has an elemental composition of $\left[\mathrm{C}_{14} \mathrm{H}_{13} \mathrm{~N}_{4}\right]^{+}$, therefore it cannot be residual dipyridyl-tetrazine 2a. (B) Background substracted mass spectrum for the reaction of chlofentezine (2b) to give $\mathbf{3 b}$.

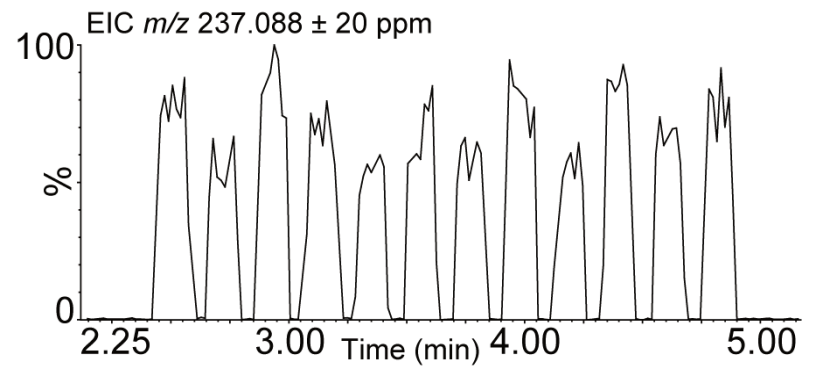

Figure S12. EIC of dipyridyl-tetrazine 2a analysis in regular LAESI conditions (without click reagent in ESI spray). Concentration of $2 \mathbf{a}$ was $1 \mathrm{mM}$ in $\mathrm{MeOH}-\mathrm{H}_{2} \mathrm{O}(1: 1)$ in the well plate, analysis parameters were as described in experimental, with a few exceptions; no reaction tube or ESI spray reagents were used. 


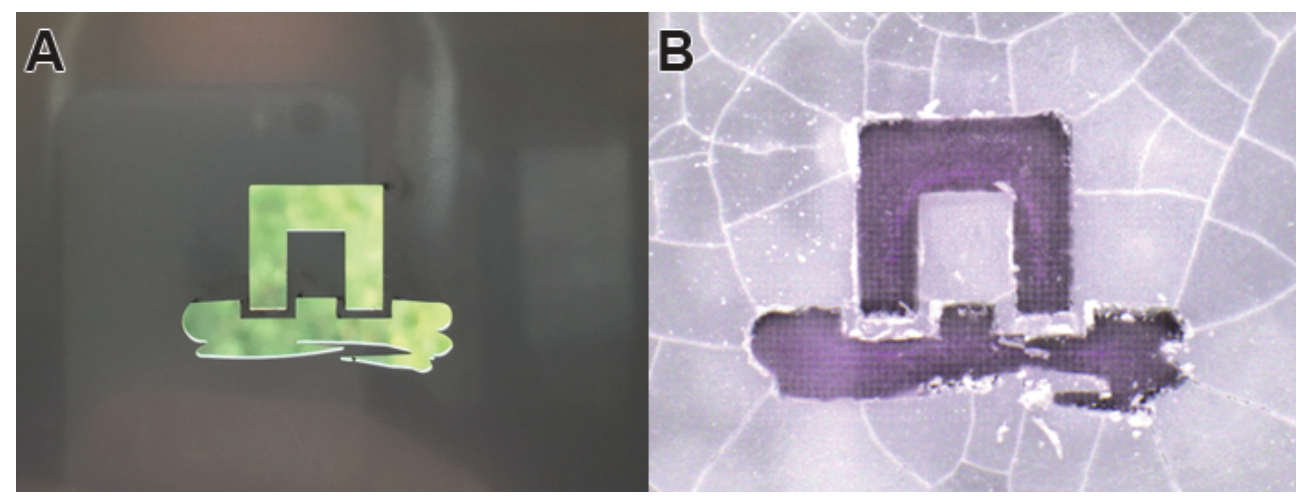

Figure S13. Template and created sample of Wageningen University logo. (A) Template $(20 \mathrm{~mm} \times 29 \mathrm{~mm})$ used to create the Wageningen University logo. (B) Optical image of the sample as analyzed in Figure 7. 


\section{Chapter 4}

\section{$\mathrm{TiO}_{2}$ Photocatalyzed Oxidation of Drugs Studied by Laser Ablation Electrospray Ionization Mass Spectrometry}
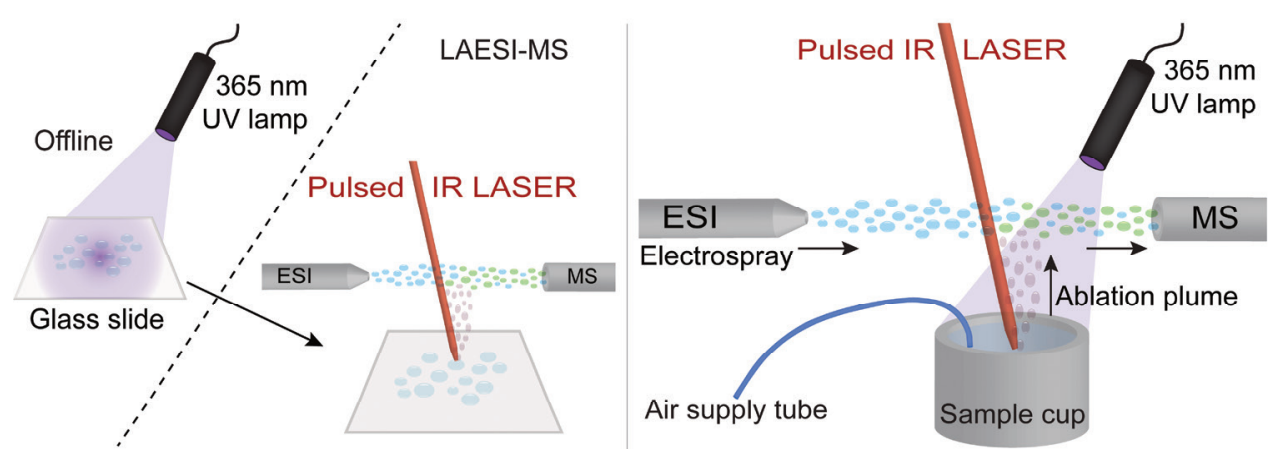

This Chapter was published as:

Fred A. M. G. van Geenen, Maurice C. R. Franssen, Ville Miikkulainen, Mikko Ritala, Han Zuilhof, Risto Kostiainen and Michel W. F. Nielen. $\mathrm{TiO}_{2}$ Photocatalyzed Oxidation of Drugs Studied by Laser Ablation Electrospray Ionization Mass Spectrometry. Journal of The American Society for Mass Spectrometry, 2019, 30, 639-646. 


\section{Abstract}

In drug discovery it is important to identify phase I metabolic modifications as early as possible to screen for inactivation of drugs and/or activation of prodrugs. As the major class of reactions in phase I metabolism are oxidation reactions, oxidation of drugs with $\mathrm{TiO}_{2}$ photocatalysis can be used as a simple non-biological method to initially eliminate (pro)drug candidates with an undesired phase I oxidation metabolism. Analysis of reaction products is commonly achieved with mass spectrometry coupled to chromatography. However, sample throughput can be substantially increased by eliminating pretreatment steps and exploiting the potential of ambient ionization mass spectrometry (MS). Furthermore, online monitoring of reactions in a time-resolved way would identify sequential modification steps. Here we introduce a novel (time-resolved) $\mathrm{TiO}_{2}$-photocatalysis laser ablation electrospray ionization (LAESI) MS method for the analysis of drug candidates. This method was proven to be compatible with both $\mathrm{TiO}_{2}$-coated glass slides as well as solutions containing suspended $\mathrm{TiO}_{2}$ nanoparticles, and the results were in excellent agreement with studies on biological oxidation of verapamil, buspirone, testosterone, andarine, and ostarine. Finally, a time-resolved LAESI MS setup was developed and initial results for verapamil showed excellent analytical stability for online photocatalyzed oxidation reactions within the set-up up to at least one hour. 


\section{Introduction}

Metabolism of drugs is an important aspect of their efficacy after administration to the body. One aspect of human metabolism is the excretion of xenobiotics - e.g. drugs or carcinogens - by biochemical transformations. In pharmacology, these transformations can also be exploited for the activation of prodrugs. The metabolism of drugs is often divided into two phases, biotransformation and bioconjugation, respectively. The biotransformation, phase I, plays an important role in the deactivation of drugs and activation of prodrugs, and comprises reactions such as oxidation, reduction, and hydrolysis. In drug discovery, it is important to identify these modifications as early as possible to screen for possible pharmacologically active compounds. ${ }^{1,2}$ Commonly, the metabolism of drug candidates is evaluated by in vitro biotransformations (with microsomes or recombinant enzymes), which are time-consuming and relatively expensive. ${ }^{2}$ Since the major class of reactions in phase I metabolism are oxidation reactions, ${ }^{3}$ also non-enzymatic methods could be used for rapid phase I reactions to eliminate drug candidates with an undesired metabolism. Apart from electrochemistry approaches, ${ }^{4,5}$ titanium dioxide $\left(\mathrm{TiO}_{2}\right)$ photocatalysis is a simple nonenzymatic method to generate oxidation reaction products similar to those obtained by phase I biochemical transformations. ${ }^{6-13}$

In $\mathrm{TiO}_{2}$ photocatalysis (see refs ${ }^{14}$ and ${ }^{15}$ for reviews), $\mathrm{TiO}_{2}$ is used as a material that is able to catalyze oxidation and reduction reactions when exposed to ultra-violet (UV) radiation. ${ }^{16-18}$ The absorbance of a photon by $\mathrm{TiO}_{2}$ with an energy greater than its bandgap ( $3.2 \mathrm{eV}, 385 \mathrm{~nm}$ ) will excite a valence electron, producing a free electron in the conduction band and leaving a hole in the valence band. Both the hole and free electron can react with other molecules that are present at the material surface, like molecular oxygen and water molecules. Molecular oxygen can be reduced to superoxide $\left(\mathrm{O}_{2}^{-}\right)$, which is a very strong oxidizing agent of organic molecules. ${ }^{19}$ In both the oxidation (by photogenerated holes) and reduction (by the free electrons) of water molecules hydroxyl radicals are produced. Hydroxyl radicals are highly oxidative and has been used extensively as a tool for oxidation reactions with drug candidates. ${ }^{6-13}$

Mass spectrometry (MS), often coupled with liquid chromatography (LC), is a wellestablished analysis technique for the identification and quantification of drugs and their metabolites following in vitro and in vivo studies. Hence, LC-MS has been used as analytical method of choice for identification of the drug reaction products in most $\mathrm{TiO}_{2}$ photocatalyzed oxidation studies. ${ }^{6-12}$ This method requires the removal of $\mathrm{TiO}_{2}$ particles priori to LC-MS analysis, introducing pretreatment steps and thus limiting sample throughput. Improvements have been achieved with a nanoreactor electrospray ionization chip $\left(\mathrm{TiO}_{2}-\mu \mathrm{PESI}\right)$ containing integrated $\mathrm{TiO}_{2}$-coated micropillars for direct $\mathrm{MS}$ analysis. ${ }^{13}$ Apart from this approach, a simple $\mathrm{TiO}_{2}$-coated rotating platform was recently introduced in combination with desorption electrospray ionization (DESI) MS, providing the opportunity to increase throughput of 
oxidation reactions up to four samples per minute. ${ }^{20}$ Although these MS methods do not allow for the separation of isomeric reaction products, MS/MS provides information on the modification site. A drawback in current $\mathrm{TiO}_{2}$ photocatalysis systems is the possibility of continuous oxidation of formed reaction products, as often multiple, consecutive oxidation reactions might take place. This makes it difficult to identify sequential oxidation steps. An online, time-resolved based method would therefore be a real asset for identification of products, and to follow consecutive and/or parallel modifications in real time.

Laser ablation electrospray ionization (LAESI) is an ambient MS technique used in the analysis of, e.g., tissues, food contaminants, solid materials, and liquids. ${ }^{21-29}$ It uses a 2.94 $\mu \mathrm{m}$ mid-infrared pulsed laser that addresses hydroxyl moieties leading to the efficient ablation of sample material that is subsequently extracted by charged electrospray droplets upon MS analysis. LAESI has previously been demonstrated its ability to allow monitoring of enzymatic reactions in time, directly from a well plate. ${ }^{28}$ In a $\mathrm{LAESI} \mathrm{TiO}_{2}$ photocatalysis $\mathrm{MS}$ system, reaction products in the ablation plume would simply be extracted by electrospray droplets for MS detection, without any need for removal of $\mathrm{TiO}_{2}$ particles. Temporal resolution would then easily be obtained by online UV exposure inside the LAESI ambient ionization source, providing the opportunity to study reactions in real time.

In the present work we introduce LAESI-MS as an analysis technique in $\mathrm{TiO}_{2}$ photocatalysis. The feasibility of the method is demonstrated and critically compared to LCMS, $\mathrm{TiO}_{2}-\mu \mathrm{PESI}-\mathrm{MS}$, and DESI-MS $\mathrm{TiO}_{2}$ photocatalysis methods for verapamil, buspirone, and testosterone. Furthermore, the method is used to generate reaction products of the selective androgen receptor modulators andarine and ostarine, and compared with metabolites obtained in vitro. Finally, time-resolved $\mathrm{TiO}_{2}$ photocatalysis $\mathrm{LAESI-MS}$ is shown, with the online $\mathrm{TiO}_{2}$ photocatalyzed oxidation reaction of verapamil as a model system.

\section{Experimental section}

\section{Materials}

$\mathrm{TiO}_{2}$-coated glass slides were created by atomic layer deposition of titanium(IV) isopropoxide on glass; the full procedure has been reported elsewhere. ${ }^{20}$ Verapamil, buspirone and titanium(IV) oxide nanopowder P25 were obtained from Sigma-Aldrich (Zwijndrecht, The Netherlands). Testosterone, ostarine and andarine were kindly donated by RIKILT (Wageningen, The Netherlands). Ultrapure water $\left(\mathrm{H}_{2} \mathrm{O}\right)-18.2 \mathrm{M} \Omega \times \mathrm{cm}^{-1}$ at $25^{\circ} \mathrm{C}-$ was freshly produced daily with a Millipore (Molsheim, France) Integral 3 system. Acetonitrile (ACN) LC-MS grade was bought from VWR (Leuven, Belgium). Methanol (MeOH) UPLC-MS grade was purchased from Biosolve (Valkenswaard, The Netherlands). Formic acid (FA) LCMS grade was bought from Fisher Scientific (Geel, Belgium) and leucine-enkephalin (leu-enk) for lock mass-corrected mass calibration was purchased from Waters (Manchester, UK). 


\section{LAESI-MS of $\mathrm{TiO}_{2}$ Photocatalyzed Oxidation Reactions on Glass Slides}

For all compounds only $1 \mu \mathrm{L}$ of sample solution $(1 \mathrm{mM})$ was dispensed on $\mathrm{TiO}_{2}-$ coated glass slides. The sample spots were UV exposed until dry - roughly between 60 and $180 \mathrm{~s}$, depending on solvent volatility - with a Uvet (Guangdong, P.R. China) UV LED Spot Curing System NSC4 (specified maximum peak at $365 \mathrm{~nm}$, intensity $650 \mathrm{~mW} / \mathrm{cm}^{2}$ at $4 \mathrm{~cm}$ distance). $2 \mu \mathrm{L}$ solvent was added to the dried sample spots before direct analysis with a Protea Biosciences (Morgantown, WV) LAESI DP-1000 system coupled to a Waters Synapt G2S traveling wave ion mobility (IMS) time of flight (TOF) mass spectrometer (MS). LAESI desktop software v.2.0.1.3 (Protea Biosciences) was used to control experimental parameters of the LAESI system. The Nd:YAG optical parametric oscillator mid-infrared laser $(2.94 \mu \mathrm{m})$ was set to $100 \%$ laser power $\left(\Phi 3.2 \mathrm{~J} / \mathrm{cm}^{2}\right)$ and 10 pulses with a specified pulse length of 5 ns were acquired on every spot $(\varnothing 200 \mu \mathrm{m})$ at a frequency of $5 \mathrm{~Hz}$. A solution of $\mathrm{MeOH}: \mathrm{H}_{2} \mathrm{O}$ (1:1) with $0.1 \% \mathrm{FA}$ and $40 \mathrm{ng} \times \mathrm{mL}^{-1}$ leu-enk was used as electrospray solvent in positive ion mode whereas in negative ion mode a solution of $\mathrm{MeOH}: \mathrm{H}_{2} \mathrm{O}(1: 1)$ with $100 \mathrm{ng} \times \mathrm{ml}^{-1}$ leu-enk was used. Electrospray flow rate was $1 \mu \mathrm{l} \times \mathrm{min}^{-1}$ and the voltage was set at $\sim 3.5 \mathrm{kV}$ (ESI+) or $3.0 \mathrm{kV}$ (ESI-) in order to have a stable ESI signal. The Synapt G2S was controlled by MassLynx v4.1 SCN 883 (Waters) and operated in either positive - for verapamil, buspirone and testosterone - or negative - for ostarine and andarine - ion TOF-MS resolution mode, $\mathrm{m} / \mathrm{z}$ range $50-1200$, scan time $1 \mathrm{~s}$, and source and interface temperatures were both set at $150{ }^{\circ} \mathrm{C}$. Background-subtracted mass spectra were generated using the "combine spectrum" function in MassLynx: five scans, each corresponding to five laser pulses of UV exposed sample spots were combined and fifty scans, matching laser pulses of non-UV exposed sample spots (blank) together with electrospray background, were subtracted.
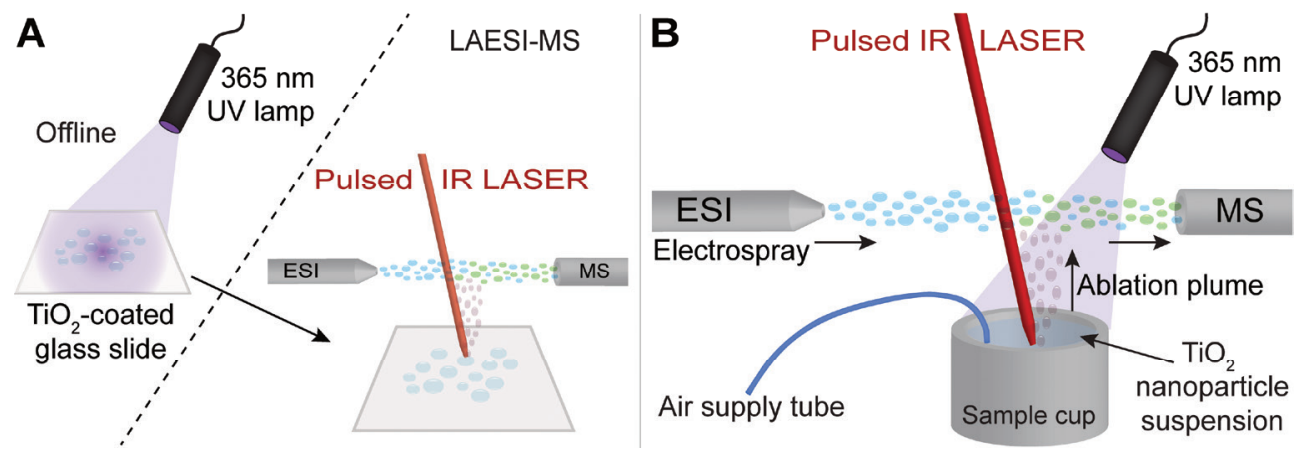

Figure 1. Schematic overview of $\mathrm{TiO}_{2}$ photocatalysis $\mathrm{LAESI-MS}$. A depicts offline UV exposure of samples on $\mathrm{TiO}_{2}-$ coated glass slides followed by direct LAESI-MS analysis. B shows the online time-resolved $\mathrm{TiO}_{2}$ photocatalysis LAESIMS setup, comprising an ESI probe, a sample cup with PEEK tube for oxygen supply, a mid-IR laser, and a $365 \mathrm{~nm}$ UV lamp. Additional details can be found in the experimental section and a picture of $\mathbf{B}$ is shown in Figure S1. 


\section{Time-Resolved $\mathrm{TiO}_{2}$ Photocatalysis LAESI-MS}

For the time-resolved measurements the UV lamp was installed inside the LAESI system. Verapamil $(1 \mathrm{mM})$ was dissolved in $0.2 \mathrm{~g} \times \mathrm{L}^{-1} \mathrm{TiO}_{2}$ nanopowder $\left(\mathrm{H}_{2} \mathrm{O}\right)$ and the polypropylene sample cup containing the solution $(4 \mathrm{~mL})$ was mounted inside the LAESI system. PEEK tubing, connected to an air supply was placed into the solution to provide a continuous oxygen source as well as a stirring mechanism due to the bubbling of air (5 $\left.\mathrm{mL} \times \mathrm{min}^{-1}\right)$. An overview of the experimental setup is shown in Figure 1B. The sample was online UV exposed for $60 \mathrm{~min}$, during which the mid-IR laser was pulsed continuously at a frequency of $0.1 \mathrm{~Hz}$, and MS scan time was $0.5 \mathrm{~s}$. The heated MS inlet temperature was decreased to $80^{\circ} \mathrm{C}$ to minimize solvent evaporation in the sample cup. Extracted ion current (EIC) signals were integrated with MassLynx and the (laser ablation-induced) peak areas obtained were normalized to the verapamil $[\mathrm{M}+\mathrm{H}]^{+}$signal.

\section{Results and Discussion}

\section{LAESI-MS as an Analysis $\mathrm{Tool}$ in $\mathrm{TiO}_{2}$ Photocatalyzed Oxidation Reactions on Glass Slides}

LAESI-MS was examined as a direct analysis tool in $\mathrm{TiO}_{2}$ photocatalysis; the generated oxidation products were critically compared with data of previously reported oxidation products of the model compounds verapamil, buspirone and testosterone. $7,10,11,13,20$ A first notable practical aspect was that LAESI, like DESI, was able to easily analyze samples on surfaces without any sample pre-treatment or lengthy chromatographic separation. Oxidation products were generated by simple offline UV exposure of analyte solutions on $\mathrm{TiO}_{2}$-coated glass slides, after which LAESI analysis directly onto the glass slides proved feasible. Additional analyte solution spots on the same glass slides were covered to prevent UV exposure; the obtained LAESI-MS signals of these spots were used for MS background subtraction. Table 1 presents the obtained photocatalyzed oxidation products of verapamil together with their collision-induced dissociation (CID) MS/MS product ions. Detected LAESIionized oxidation products at $\mathrm{m} / \mathrm{z} 291.206,441.270,487.282$ and 469.266 correspond to the molecular structures $[\mathrm{M}-164+\mathrm{H}]^{+}\left(\mathrm{N}\right.$-dealkylation), $\left[\mathrm{M}-\mathrm{CH}_{2}+\mathrm{H}\right]^{+}$(demethylation), $[\mathrm{M}+2 \mathrm{O}+\mathrm{H}]^{+}$ (dihydroxylation) and $\left[\mathrm{M}+\mathrm{O}-\mathrm{H}_{2}+\mathrm{H}\right]^{+}$(carbonyl formation), respectively. Both the demethylation $\left(\mathrm{M}-\mathrm{CH}_{2}\right)$ and carbonyl formation $\left(\mathrm{M}+\mathrm{O}-\mathrm{H}_{2}\right)$ appear to occur at various positions in the molecule. For the demethylation this is shown by the CID product ions $\mathrm{m} / \mathrm{z} 151.076$ and $\mathrm{m} / \mathrm{z}$ 289.192 (Table 1, patterns $\mathbf{B}$ and $\mathbf{C}$, respectively) that both comprise $\mathrm{CH}_{2}$ (-14.02 Da.) loss. In the carbonyl formation - oxidation product - this is indicated by the two different CID product ions $\mathrm{m} / \mathrm{z} 179.070$ and $\mathrm{m} / \mathrm{z} 317.187$ (Table 1, patterns B and C, respectively), which show a $13.98 \mathrm{Da}$ addition $\left(+\mathrm{O}-\mathrm{H}_{2}\right)$ compared to their verapamil equivalents. The observed photocatalyzed oxidation products and CID product ions are similar to those reported in $\mathrm{TiO}_{2}$ - 
$\mu \mathrm{PESI}$ and DESI analysis studies. ${ }^{13,20}$ One exception is a product detected with $\mathrm{TiO}_{2}-\mu \mathrm{PESI}-$ MS at $m / z 195$ (N-dealkylation) which was not observed in our data. This absence could be a result of the specific $\mathrm{TiO}_{2}$ photocatalysis procedure used, as with $\mathrm{TiO}_{2}-\mu \mathrm{PESI}$ the photocatalytic micropillar nanoreactor was integrated in the electrospray ionization chip, which comprises a different setup. Our method has more similarities with the DESI study, in which $m / z 195$ was absent as well. ${ }^{20}$ The obtained verapamil CID fragmentation pathway in Table 1 is similar to the one suggested by Walles et al. ${ }^{30}$

\begin{tabular}{|c|c|c|c|c|c|c|c|}
\hline \multirow{2}{*}{$\begin{array}{l}\text { Photocatalytic } \\
\text { oxidation product }\end{array}$} & \multirow[b]{2}{*}[\mathrm{M}+\mathrm{H}]{$^{+}$} & \multicolumn{6}{|c|}{ Observed CID MS/MS product ions $(\mathrm{m} / \mathrm{z})$} \\
\hline & & A & $\mathrm{B}$ & $\mathrm{C}$ & $\mathrm{D}$ & $\mathrm{E}$ & other \\
\hline Verapamil & 455.290 & 150.068 & 165.091 & 303.206 & & 260.165 & \\
\hline $\begin{array}{l}\text { M-164, } N- \\
\text { dealkylation }\end{array}$ & 291.206 & & & & & 260.165 & $\begin{array}{l}248.152 \\
177.091 \\
165.091 \\
151.076\end{array}$ \\
\hline $\mathrm{M}-\mathrm{CH}_{2}$ & 441.270 & 150.068 & $\begin{array}{l}165.092 \\
151.076\end{array}$ & $\begin{array}{l}303.207 \\
289.192\end{array}$ & 291.207 & 260.165 & \\
\hline $\mathrm{M}+2 \mathrm{O}$ & 487.282 & & 165.092 & $\begin{array}{l}303.208 \\
289.192\end{array}$ & & 260.165 & $\begin{array}{l}469.270 \\
440.267\end{array}$ \\
\hline $\mathrm{M}+\mathrm{O}-\mathrm{H}_{2}$ & 469.266 & & $\begin{array}{l}179.070 \\
165.091 \\
151.076\end{array}$ & $\begin{array}{l}303.207 \\
317.187\end{array}$ & & 260.166 & \\
\hline
\end{tabular}

Proposed CID fragmentation pattern of verapamil

Table 1. Observed $\mathrm{TiO}_{2}$ photocatalyzed oxidation products of verapamil, as generated on $\mathrm{TiO}_{2}$-coated glass slides, together with their CID product ions. A background-subtracted mass spectrum is given in Figure S2A and CID MS/MS spectra of verapamil and photocatalyzed oxidation products are provided in Figure S3.

For buspirone the obtained $\mathrm{TiO}_{2}$ photocatalyzed oxidation products following LAESIionization were $\mathrm{m} / \mathrm{z} 402.254,400.241,384.240$ and 418.253 , and are presented along with the their CID MS/MS product ions in Table 2. The obtained oxidation products relate to the molecular structures $[\mathrm{M}+\mathrm{O}+\mathrm{H}]^{+}$(hydroxylation), $\left[\mathrm{M}+\mathrm{O}-\mathrm{H}_{2}+\mathrm{H}\right]^{+}$(hydroxylation and dehydrogenation), $\left[\mathrm{M}-\mathrm{H}_{2}+\mathrm{H}\right]^{+}$(dehydrogenation) and $[\mathrm{M}+2 \mathrm{O}+\mathrm{H}]^{+}$(dihydroxylation), respectively. As with verapamil, all obtained oxidation products are in excellent agreement with LC-MS and DESI-MS studies on this compound, underlining the feasibility of LAESI-MS as a rapid analysis tool for $\mathrm{TiO}_{2}$ photocatalysis. ${ }^{7,11,20}$ Furthermore, the obtained CID product ions $\mathbf{D}$ and $\mathbf{F}$ (Table 2$)$ of photocatalyzed oxidation products $\mathrm{M}+\mathrm{O}(\mathrm{m} / \mathrm{z} 402.254)$ and $\mathrm{M}+\mathrm{O}-\mathrm{H}_{2}$ $(m / z$ 400.241) in particular demonstrate distinctive CID fragmentation. The hydroxylation 
$(\mathrm{M}+\mathrm{O})$ product suggests a rapid loss of $\mathrm{H}_{2} \mathrm{O}$ upon $\mathrm{CID}$, making the hydroxylation site undetected in CID MS/MS analysis from the $\mathrm{m} / \mathrm{z}$ values of fragment ions $\mathbf{D}$ and $\mathbf{F}$. However, with the hydroxylation and dehydrogenation $\left(\mathrm{M}+\mathrm{O}-\mathrm{H}_{2}\right)$ product this site is indicated to be in CID product ion $\mathbf{F}$ due to the increased $\mathrm{m} / \mathrm{z}$ value of this CID product ion (from $\mathrm{m} / \mathrm{z} 265$ to 279). The dehydrogenation site is located in the piperazine moiety, as indicated by CID MS/MS product ion $\mathbf{E}(\mathrm{m} / \mathrm{z} 162.090$, Table 2$)$. The opportunity to include CID MS/MS is a useful option, but a minor shortcoming of ambient ionization MS compared to hyphenated-MS techniques is the absence of the extra separation dimension. E.g., there were four chromatographic peaks reported by Calza et al. in the LC-MS analysis of hydroxy-buspirone ( $\mathrm{m} / \mathrm{z} 402$ ), corresponding to at least four different isobaric hydroxylation products. ${ }^{7} \mathrm{An}$ additional separation dimension compatible with ambient ionization MS is ion mobility (IMS). ${ }^{26}$ The applicability of IMS was investigated by calculation of collision cross sections (CCS) for reported isobaric hydroxylation products and are provided in Table S1. ${ }^{7}$ Buspirone hydroxylation products are varying in CCS by circa $1 \%$, whereas the IMS resolving power of used instrumentation is capable of separating compounds with CCS differences of over approximately $5 \% .{ }^{31}$ The IMS separation of buspirone hydroxylation products are therefore beyond the resolving power of present instrumentation and was not further investigated here. Nonetheless, studies comprising other molecules and/or different ion mobility hardware could benefit from separation with this technique. The CID MS/MS fragmentation pathway as proposed in Table 2 is in good agreement with literature. ${ }^{7,32}$

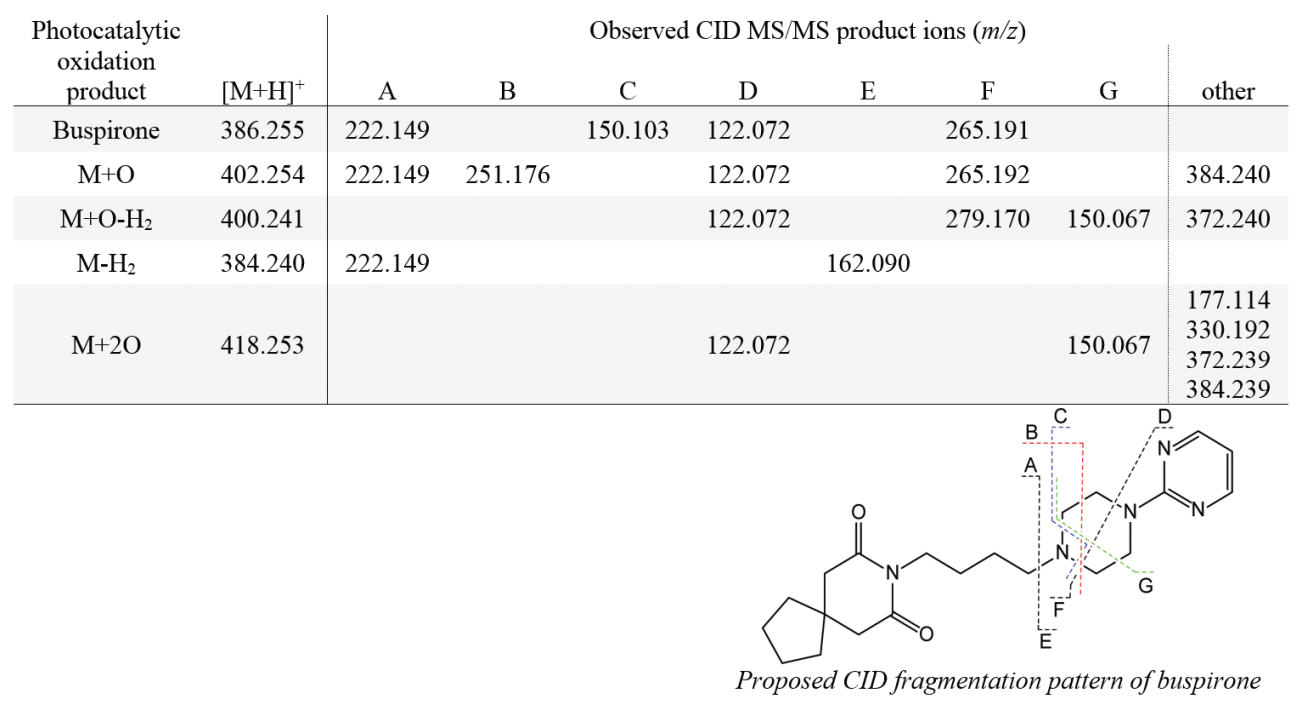

Table 2. Observed $\mathrm{TiO}_{2}$ photocatalyzed oxidation products of buspirone, and their CID MS/MS product ions. A background-subtracted mass spectrum is given in Figure S2B and CID MS/MS spectra of buspirone and photocatalyzed oxidation products are provided in Figure S4. 
Finally the steroid testosterone was studied to show the feasibility of $\mathrm{TiO}_{2}$ photocatalysis LAESI-MS for analysis of a more hydrophobic compound. Testosterone was dissolved in $\mathrm{H}_{2} \mathrm{O}: \mathrm{ACN}(1: 1 \mathrm{v} / \mathrm{v})$ priori to the analysis. $\mathrm{ACN}$ is known to be an $\mathrm{OH}$ radical scavenger and may therefore somewhat hamper the $\mathrm{TiO}_{2}$ photocatalyzed oxidation of testosterone. ${ }^{10,33}$ Despite this organic modifier, oxidation products were easily obtained, and are presented with their CID MS/MS product ions in Table S2. The observed reaction products $\mathrm{M}+\mathrm{O}\left(\mathrm{m} / \mathrm{z}\right.$ 305.209), $\mathrm{M}+\mathrm{O}-\mathrm{H}_{2}\left(\mathrm{~m} / \mathrm{z}\right.$ 303.194) and $\mathrm{M}-\mathrm{H}_{2}(\mathrm{~m} / \mathrm{z} 287.203)$ are in line with the products detected in conventional LC-MS analysis. ${ }^{10,11}$

\section{$\mathrm{TiO}_{2}$ Photocatalysis LAESI-MS of Selective Androgen Receptor Modulators on Glass Slides}

Following the verification of LAESI-MS as a suitable analysis tool in $\mathrm{TiO}_{2}$ photocatalysis on glass slides, the method was used to study the oxidation products of the selective androgen receptor modulators andarine and ostarine. The only photocatalytic reaction product of andarine was observed at $\mathrm{m} / \mathrm{z} 307.058$ (Figure $2 \mathrm{~A}$ ) following LAESI-MS analysis, and the proposed mechanism is depicted in Figure S6. The proposed structure could further be confirmed by MS/MS of $m / z 307.058$, via the observation of a fragment ion at $\mathrm{m} / \mathrm{z}$ 205.023. This $m / z 307.058$ peak and similar MS/MS spectra were reported to belong to the most abundant metabolite in several biological in vitro and in vivo studies, using human liver microsomes and human urine. ${ }^{34-40}$ In those studies, reported phase II metabolites produced by enzymes such as glutathione-S-transferase, $\mathrm{N}$-acetyl transferase, and sulfotransferase, are obviously not observed in $\mathrm{TiO}_{2}$ photocatalyzed oxidation studies.

In case of ostarine, the LAESI-ionized oxidation products were observed at $\mathrm{m} / \mathrm{z}$ 287.062 and $m / z 404.093$ (Figure $2 \mathrm{~B}$ ). The ion at $\mathrm{m} / \mathrm{z} 287.062$ is proposed to be produced following a similar reaction mechanism (Figure S6) as $\mathrm{m} / z 307.058$ of andarine. The structure is further confirmed by MS/MS to yield a CID fragment ion at $m / z 185.033$, which is in agreement with literature. ${ }^{40,41}$ The oxidation product appearing at $\mathrm{m} / \mathrm{z} 404.093$ is most likely formed by hydroxylation of ostarine. Multiple hydroxylation sites were identified by MS/MS experiments. The most obvious sites are aromatic rings (Figure S7). The CID fragment ions at $m / z 285.049$ and $m / z 118.030$ correspond to hydroxylation in aromatic ring $\mathbf{A}$, whereas the fragment ions at $\mathrm{m} / \mathrm{z} 269.054$ and $\mathrm{m} / \mathrm{z} 134.025$ associate with hydroxylation at ring $\mathbf{B}$. A fragmentation mechanism for these compounds has been reported previously. ${ }^{33}$ However, we here propose a different CID fragmentation mechanism, leading to fragment ions with a stable $\mathrm{C}-\mathrm{N}$ bond instead of a labile $\mathrm{N}-\mathrm{O}$ bond (Figure S7B). These results demonstrate that our method provides rapid identification of photocatalyzed oxidation products that mimic products found in several in vitro studies. ${ }^{34-41}$

Although both andarine and ostarine do not significantly absorb UV light at $\lambda=365$ $\mathrm{nm}$, the same experiment, but on regular - uncoated - glass slides was performed to exclude any possibility of photodegradation due to direct UV exposure. Indeed, no reaction products 
were obtained (Figure S10). Please note that all compounds used in this study do not significantly absorb UV light at $\lambda>300 \mathrm{~nm}$ and the used UV lamp does not emit UV light at $\lambda$ $<350 \mathrm{~nm}$ (Figure S11). It is therefore unlikely that any products shown in this work are a result of photodegradation.
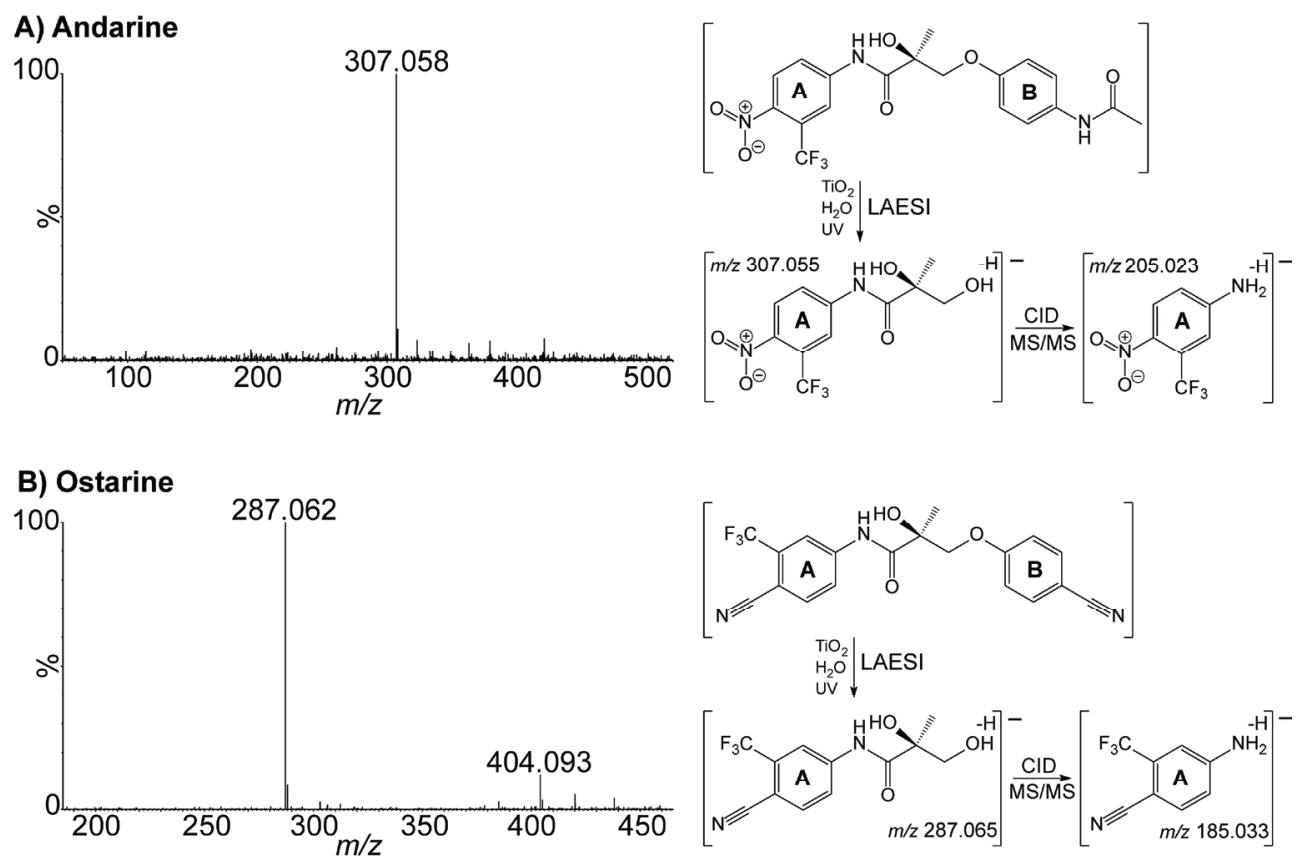

Figure 2. Background-subtracted mass spectra of the oxidation products of andarine (A) and ostarine (B), with their proposed structures. Obtained CID MS/MS spectra are provided in Figures S7 (ostarine product, $\mathrm{m} / z$ 404.093), S8 (andarine product, $\mathrm{m} / \mathrm{z} 307.058$ ), and S9 (ostarine product, $\mathrm{m} / \mathrm{z} 287.062$ ).

\section{Time-Resolved Photocatalyzed Oxidation LAESI-MS of Verapamil using $\mathrm{TiO}_{2}$ Nanoparticle Suspensions}

In order to monitor the generation of reaction products in time, an online setup was created by installing a reaction cup and the UV lamp inside the LAESI system (Figures $1 \mathrm{~B}$ and $\mathrm{S} 1$ ). Besides measuring oxidation products from $\mathrm{TiO}_{2}$-coated glass slides as demonstrated in the previous paragraphs, LAESI is also capable of measuring those directly from a sample cup containing suspended $\mathrm{TiO}_{2}$ nanoparticles in water. Verapamil was used as a model compound and the generation of several products during a reaction time of one hour is shown in Figure 3. After switching on the $365 \mathrm{~nm}$ UV lamp, at 2 min runtime, almost instantly two major products of verapamil, the $\mathrm{N}$-dealkylation and demethylation products (Figure 3 , signals $A$ and $B$ ) were observed. These reaction products steadily increased in abundance 
during the entire analysis runtime of 60 min (Figure 3, raw data in Figure S12). The added value of time-resolved analysis started to show after ca. 25 min reaction time, when a product ion at $m / z 277.19$ started to appear (Figure 3, signal C). This $m / z$ value is the result of both $N$ dealkylation and demethylation of verapamil and clearly demonstrates the power of this system in identifying subsequent oxidation steps. In addition, also the product of a double demethylation ( $\mathrm{m} / \mathrm{z}$ 427.26) was observed after ca. $18 \mathrm{~min}$ (Figure $\mathrm{S13}$ ). Following these results this time-resolved method allows the optimization of reactions conditions preliminary to high-throughput static experiments. Despite the inherent poor reproducibility of MS data from individual LAESI pulses, the reproducibility of time-resolved $\mathrm{TiO}_{2}$ photocatalyzed LAESIMS over three different analyses is actually quite good, cf. the individual lines of the $(n=3)$ averaged data presented in Figure 3 given in Figure S14. This indicates time-resolved analysis of the oxidation products of drugs is not only feasible by LAESI, but also easily adds mechanistic information that is not available upon analysis of the composition of just one time point.

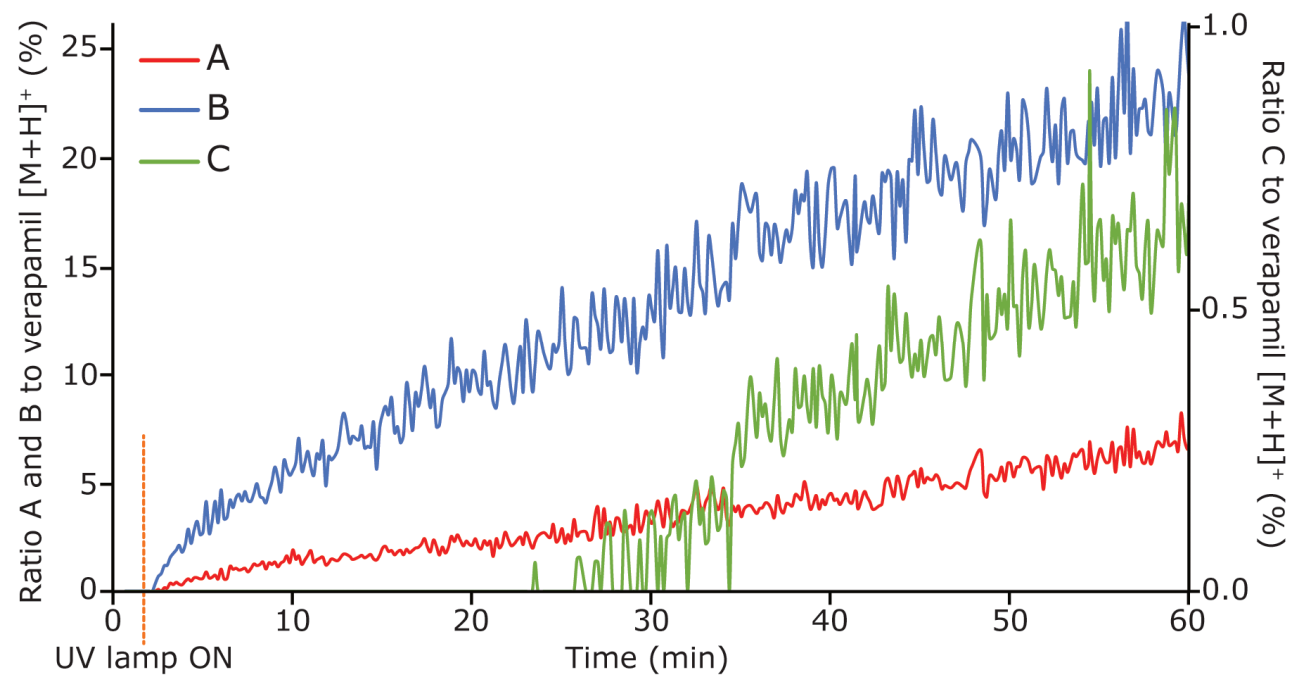

Figure 3. Time-resolved photocatalyzed oxidation LAESI-MS of verapamil using a $\mathrm{TiO}_{2}$ nanoparticle suspension. A shows the formation of the $N$-dealkylation product $(\mathrm{m} / \mathrm{z} 291.21)$ of verapamil. B displays the formation of the demethylation product of verapamil ( $\mathrm{m} / \mathrm{z} 441.28)$, and $\mathbf{C}$ shows the product of both the $N$-dealkylation and demethylation ( $\mathrm{m} / z$ 277.19), which starts to appear around $25 \mathrm{~min}$ (intensity shown at secondary $\mathrm{y}$-axis). The $365 \mathrm{~nm}$ UV lamp was switched ON after $2 \mathrm{~min}$. All lines were normalized to verapamil $[M+H]^{+}$. Data were averaged $(n=3$, for clarity, error bars are not shown), individual data are shown in Figure S14. 


\section{Conclusion}

We have developed an ambient $\mathrm{TiO}_{2}$ photocatalysis LAESI MS method to generate photocatalyzed oxidation products of drugs that are similar to phase I metabolites. Like other ambient ionization MS approaches the concept features rapid simplified analysis without any sample pretreatment or lengthy chromatographic separations. In addition, LAESI MS, was able to measure drugs and their metabolites directly from the surface of $\mathrm{TiO}_{2}$-coated glass slides as well as from $\mathrm{TiO}_{2}$ nanoparticle suspensions. Among others, the method on $\mathrm{TiO}_{2}-$ coated glass slides was demonstrated with the selective androgen receptor modulators andarine and ostarine to yield oxidation reaction products similar to those obtained in various in vitro studies. Moreover, a novel fully integrated, time-resolved LAESI MS photocatalyzed oxidation approach was successfully developed using a $\mathrm{TiO}_{2}$ nanoparticle suspension and demonstrated for verapamil. The time-resolved $\mathrm{TiO}_{2}$ photocatalysis LAESI MS exhibited excellent stability and enabled the monitoring of reaction products during a reaction time of at least one hour. This method can be used in future research to expeditiously assess drug candidates in the early stages of development as well as an online tool for time-resolved monitoring for this and of other - non- $\mathrm{TiO}_{2}$ based - reactions.

\section{Acknowledgements}

The authors thank Marco Blokland for providing reagents and preliminary discussions. The authors are grateful to receive funding for this research from The Netherlands Organization for Scientific Research (NWO) in the framework of the Technology Area TA-COAST2 of the Fund New Chemical Innovations (Project No. 053.21.111). 


\section{References}

Zhang, Z.; Tang, W. Acta Parm. Sin. $B$ 2018, DOI: https://doi.org/10.1016/j.apsb.2018.1004.1003.

Kirchmair, J.; Göller, A. H.; Lang, D., et al. Nat. Rev. Drug. Discov. 2015, 14, 387404.

Guengerich, F. P. Chem. Res. Toxicol. 2001, 14, 611-650.

Faber, H.; Vogel, M.; Karst, U. Anal. Chim. Acta 2014, 834, 9-21.

Bussy, U.; Boisseau, R.; Thobie-Gautier, C.; Boujtita, M. TrAC, Trends Anal. Chem. 2015, 70, 67-73.

Calza, P.; Pelizzetti, E.; Brussino, M.; Baiocchi, C. J. Am. Soc. Mass Spectrom. 2001, 12, 1286-1295.

Calza, P.; Pazzi, M.; Medana, C., et al. J. Pharm. Biomed. Anal. 2004, 35, 9-19.

Medana, C.; Calza, P.; Giancotti, V., et al. Anal. Bioanal. Chem. 2013, 405, 11051113.

Medana, C.; Calza, P.; Giancotti, V., et al. Drug Test. Anal. 2011, 3, 724-734.

Ruokolainen, M.; Valkonen, M.; Sikanen, T., et al. Eur. J. Pharm. Sci. 2014, 65, 45-55.

Ruokolainen, M.; Gul, T.; Permentier, H., et al. Eur. J. Pharm. Sci. 2016, 83, 3644.

Raoof, H.; Mielczarek, P.; Michalow, K. A., et al. J. Photochem. Photobiol. B 2013, $118,49-57$.

Nissilä, T.; Sainiemi, L.; Karikko, M.-M., et al. Lab Chip 2011, 11, 1470-1476.

Fujishima, A.; Zhang, X.; Tryk, D. A. Surf. Sci. Rep. 2008, 63, 515-582.

Rahimi, N.; Pax, R. A.; Gray, E. M. Prog. Solid State Chem. 2016, 44, 86-105.

Baur, E.; Neuweiler, C. Helv. Chim. Acta 1927, 10, 901-907.

Markham, M. C.; Laidler, K. J. J. Phys. Chem. 1953, 57, 363-369.

Rubin, T. R.; Calvert, J. G.; Rankin, G. T.; MacNevin, W. J. Am. Chem. Soc. 1953, 75, 2850-2853.

McLintock, I. S.; Ritchie, M. Trans. Faraday Soc. 1965, 61, 1007-1016.

Ruokolainen, M.; Miikkulainen, V.; Ritala, M., et al. Anal. Chem. 2017, 89, 1121411218.

Nemes, P.; Vertes, A. Anal. Chem. 2007, 79, 8098-8106.

Nemes, P.; Barton, A. A.; Li, Y.; Vertes, A. Anal. Chem. 2008, 80, 4575-4582.

Nemes, P.; Barton, A. A.; Vertes, A. Anal. Chem. 2009, 81, 6668-6675.

Nemes, P.; Woods, A. S.; Vertes, A. Anal. Chem. 2010, 82, 982-988.

Nielen, M. W. F.; van Beek, T. A. Anal. Bioanal. Chem. 2014, 406, 6805-6815. van Geenen, F. A. M. G.; Franssen, M. C. R.; Schotman, A. H. M., et al. Anal. Chem. 2017, 89, 4031-4037.

Nemes, P.; Huang, H.; Vertes, A. Phys. Chem. Chem. Phys. 2012, 14, 2501-2507. Nazari, M.; Ekelöf, M.; Khodjaniyazova, S., et al. Rapid Commun. Mass Spectrom. 2017, 31, 1868-1874.

van Geenen, F. A. M. G.; Franssen, M. C. R.; Zuilhof, H.; Nielen, M. W. F. Anal. Chem. 2018, 90, 10409-10416.

Walles, M.; Thum, T.; Levsen, K.; Borlak, J. J. Chromatogr. A 2002, 970, 117-130. Giles, K.; Williams, J. P.; Campuzano, I. Rapid Commun. Mass Spectrom. 2011, $25,1559-1566$.

Zhu, M.; Zhao, W.; Jimenez, H., et al. Drug Metab. Dispos. 2005, 33, 500-507. Mitroka, S.; Zimmeck, S.; Troya, D.; Tanko, J. M. J. Am. Chem. Soc. 2010, 132, 2907-2913.

Kuuranne, T.; Leinonen, A.; Schänzer, W., et al. Drug Metab. Dispos. 2008, 36, 571-581. 
Chapter 4

35 Thevis, M.; Thomas, A.; Fußhöller, G., et al. Rapid Commun. Mass Spectrom. 2010, 24, 2245-2254.

36 Krug, O.; Thomas, A.; Beuck, S., et al. J. Equine Vet. Sci. 2012, 32, 562-568.

37 Hansson, A.; Knych, H.; Stanley, S., et al. Drug Test. Analysis 2015, 7, 673-683.

38 Hansson, A.; Knych, H.; Stanley, S., et al. Rapid Commun. Mass Spectrom. 2016, 30, 833-842.

39 Rydevik, A.; Thevis, M.; Krug, O., et al. Xenobiotica 2013, 43, 409-420.

40 de Rijke, E.; Essers, M. L.; Rijk, J. C. W., et al. Food Addit. Contam. Part A 2013, 30, 1517-1526.

41 Thevis, M.; Thomas, A.; Möller, I., et al. Rapid Commun. Mass Spectrom. 2011, 25, 2187-2195. 


\section{Supporting Information Chapter 4}

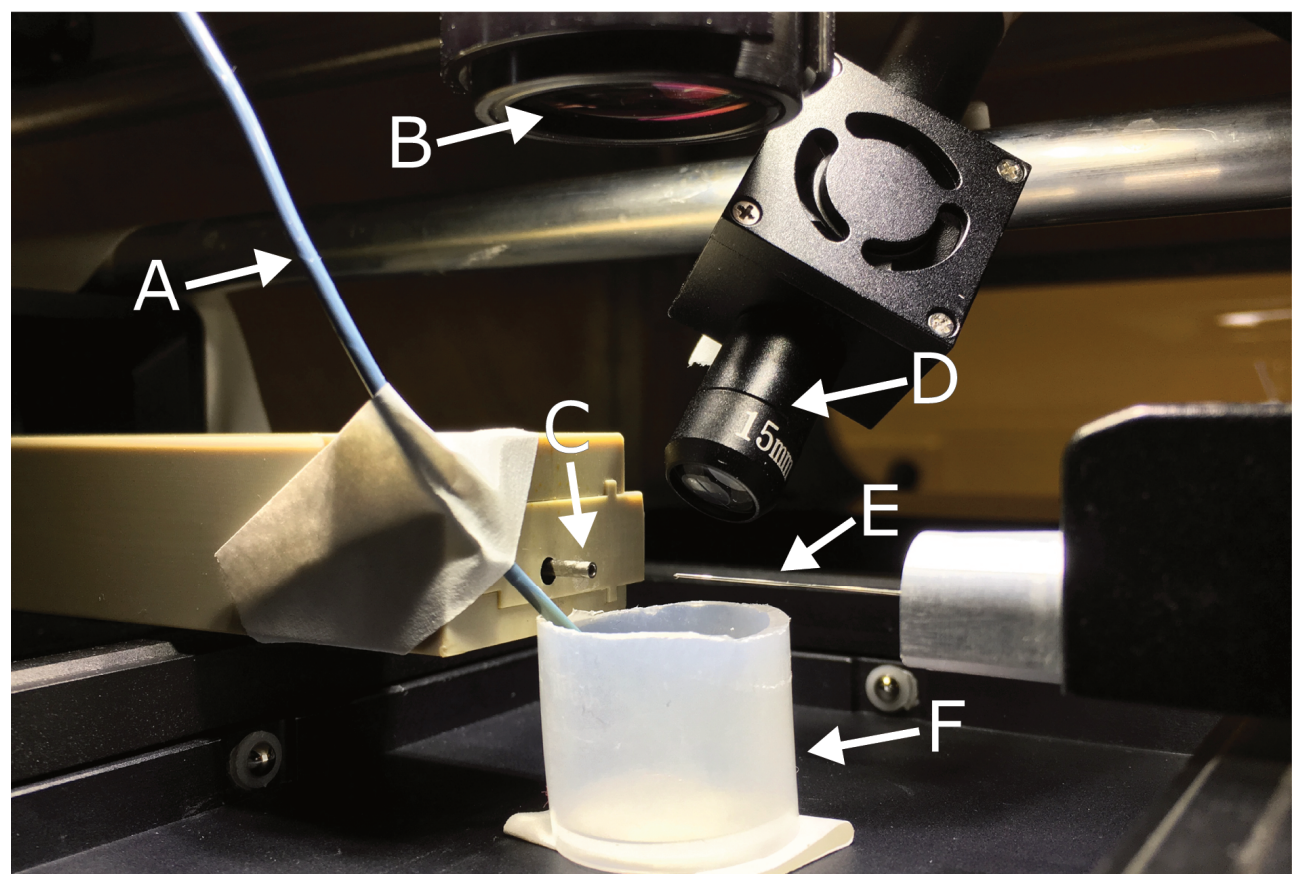

Figure S1. Picture of the time-resolved LAESI-MS setup depicted in Figure 1B. A PEEK tubing for bubbling air (oxygen supply and stirring mechanism), B mid-IR laser focussing lens, C heated MS inlet, D $365 \mathrm{~nm}$ UV lamp, E ESI spray needle and $\mathbf{F}$ polypropylene sample cup. The distance between MS inlet $\mathbf{C}$ and the ESI needle tip $\mathbf{E}$ was $15 \mathrm{~mm}$. 
Chapter 4
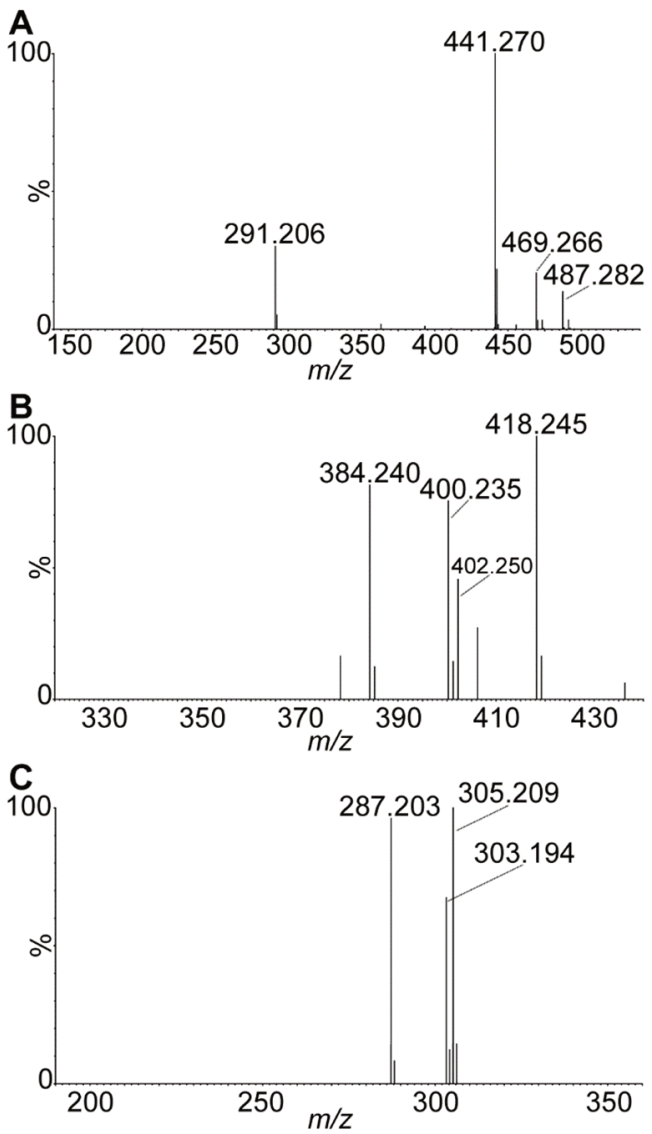

Figure S2. $\mathrm{TiO}_{2}$ photocatalyzed oxidation LAESI-MS background subtracted mass spectra of $\mathbf{A}$ verapamil, $\mathbf{B}$ buspirone and $\mathbf{C}$ testosterone on $\mathrm{TiO}_{2}$-coated glass slides. $\mathrm{CID}$ MS/MS mass spectra of observed product ions are presented in Figures S3 (verapamil), S4 (buspirone) and S5 (testosterone). For conditions, see experimental section. 

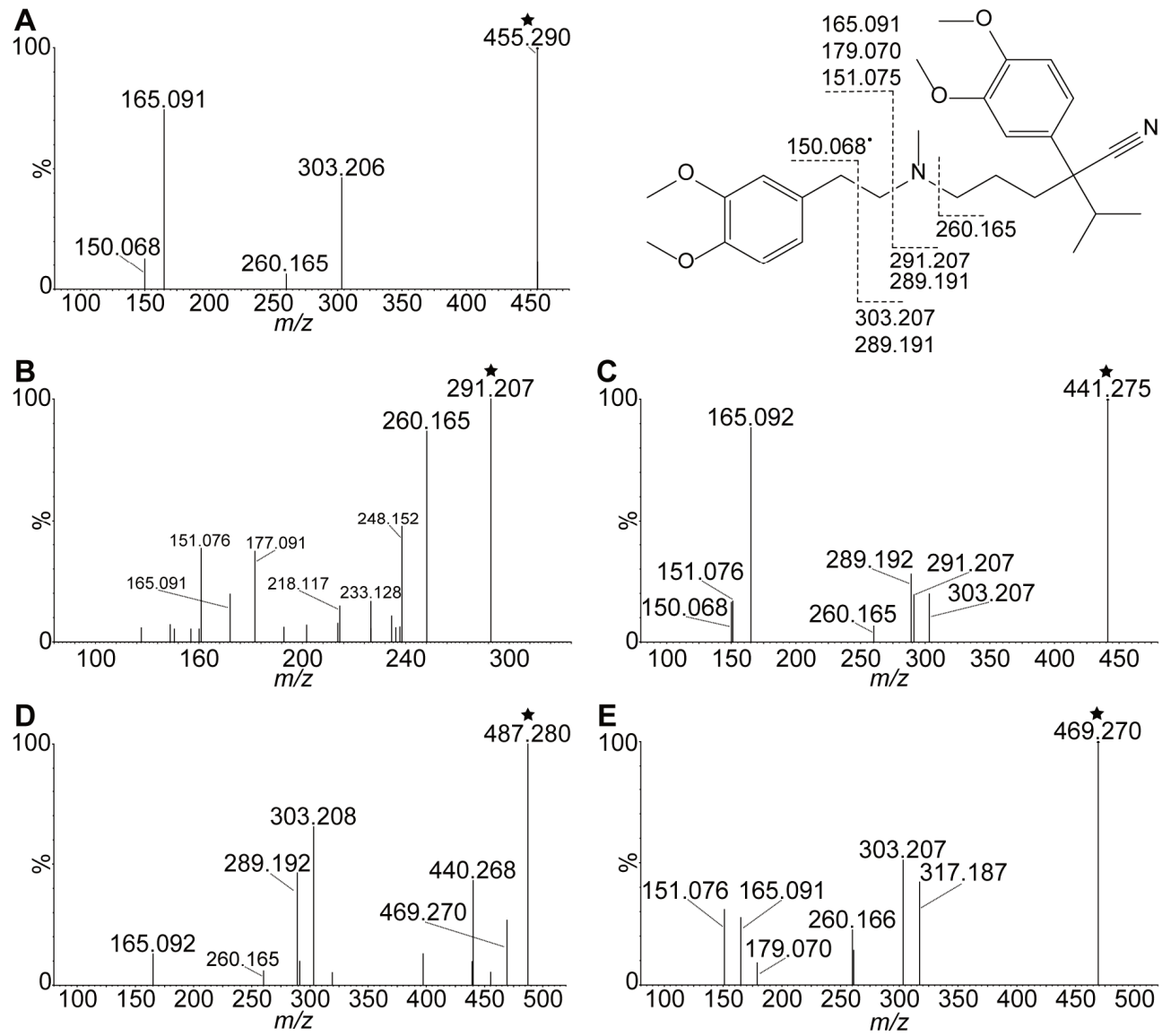

Figure S3. CID MS/MS mass spectra of verapamil (panel A) and observed $\mathrm{TiO}_{2}$ photocatalyzed oxidation products (panels B-E). Selected precursor ion is annotated with a star. The fragmentation pattern is in excellent agreement with literature. ${ }^{1,2}$ 
Chapter 4

\begin{tabular}{|l|l|l|} 
Structure & $\begin{array}{c}\text { CCS } \\
(\Omega)\end{array}$ & $\begin{array}{c}\text { Deviation (\%) from } \\
\text { average }\end{array}$ \\
\hline
\end{tabular}

Table S1. Structures and calculated collision cross sections of buspirone hydroxylation products. Structures of hydroxylation products were proposed by Calza et al. ${ }^{3}$ Hydroxy buspirone 3D structures were auto optimized using Avogadro V1.1.1 (http://avogadro.cc/) with force field set to MMFF94s. CCS values were calculated using CCSCalc (Waters), gas radius was $1.0 \AA$ and CCS tolerance $0.1 \%$. 

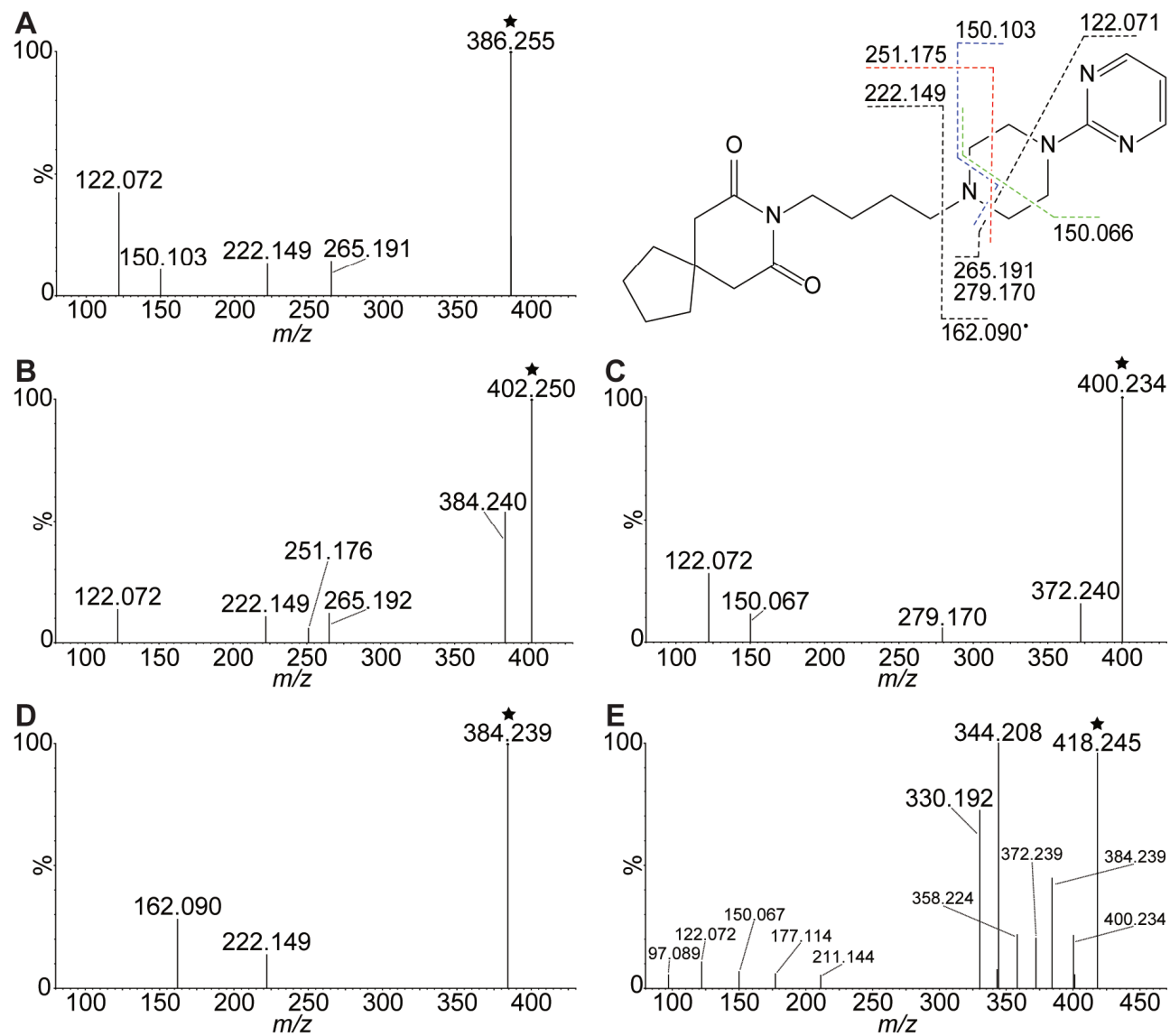

Figure S4. CID MS/MS mass spectra of buspirone (panel A) and observed $\mathrm{TiO}_{2}$ photocatalyzed oxidation products (panels B-E). Selected precursor ion is annotated with a star. The fragmentation pattern is in excellent agreement with literature..$^{3,4}$ 
Chapter 4

\begin{tabular}{|c|c|c|c|c|c|}
\hline \multirow{3}{*}{$\begin{array}{c}\text { Photocatalyzed } \\
\text { oxidation } \\
\text { product }\end{array}$} & \multirow{3}{*}{$\begin{array}{l}{[\mathrm{M}+\mathrm{H}]^{+}} \\
289.216\end{array}$} & \multicolumn{4}{|c|}{ Observed CID MS/MS product ions $(\mathrm{m} / \mathrm{z})$} \\
\hline & & A & B & \multicolumn{2}{|c|}{ other } \\
\hline & & 109.065 & 97.065 & 253.195 & 271.205 \\
\hline $\mathrm{M}-\mathrm{H}_{2}$ & 287.203 & 109.062 & 97.064 & 251.179 & 269.193 \\
\hline $\mathrm{M}+\mathrm{O}-\mathrm{H}_{2}$ & 303.194 & 109.062 & 97.064 & & 267.173 \\
\hline $\mathrm{M}+\mathrm{O}$ & 305.209 & 109.064 & 97.064 & & 269.185 \\
\hline & & . & 97.065 & Test & ne \\
\hline
\end{tabular}

Table S2. Observed photocatalyzed oxidation products of testosterone, as generated on $\mathrm{TiO}_{2}$-coated glass slides together with their CID MS/MS product ions. A background subtracted mass spectrum is given in Figure S2C and MS/MS spectra of testosterone and observed oxidation products are provided in Figure S5. 

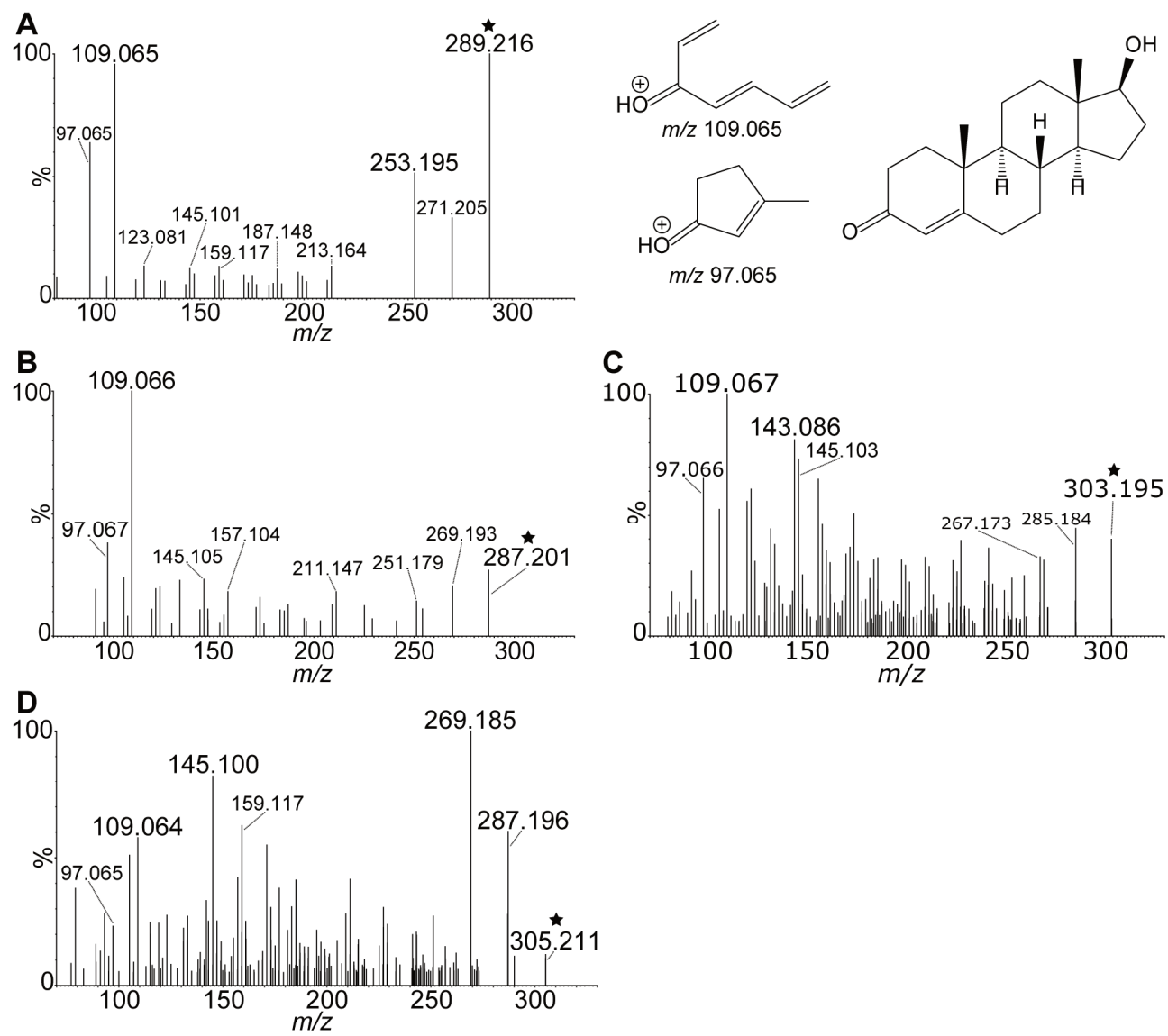

Figure S5. CID MS/MS mass spectra of testosterone (panel A) and observed $\mathrm{TiO}_{2}$ photocatalyzed oxidation products (panels B-D). Selected precursor ion is annotated with a star. The structures for $\mathrm{m} / \mathrm{z} 97$ and $\mathrm{m} / \mathrm{z} 109$ are in agreement with literature..$^{5,6}$<smiles>[R2]COc1ccc([R])cc1</smiles>
keto-enol<smiles>[R2]COC[C@]1(OC[R])C=CC([R])=CC1=O</smiles>

Figure S6. Proposed mechanism for $\mathrm{TiO}_{2}$ photocatalyzed oxidation products of andarine and ostarine, observed at $\mathrm{m} / \mathrm{z}$ 307.058 and $m / z 287.062$, respectively. 
A 100

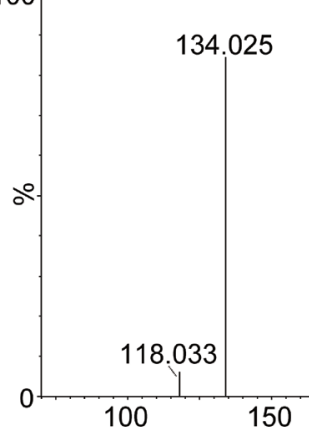

B

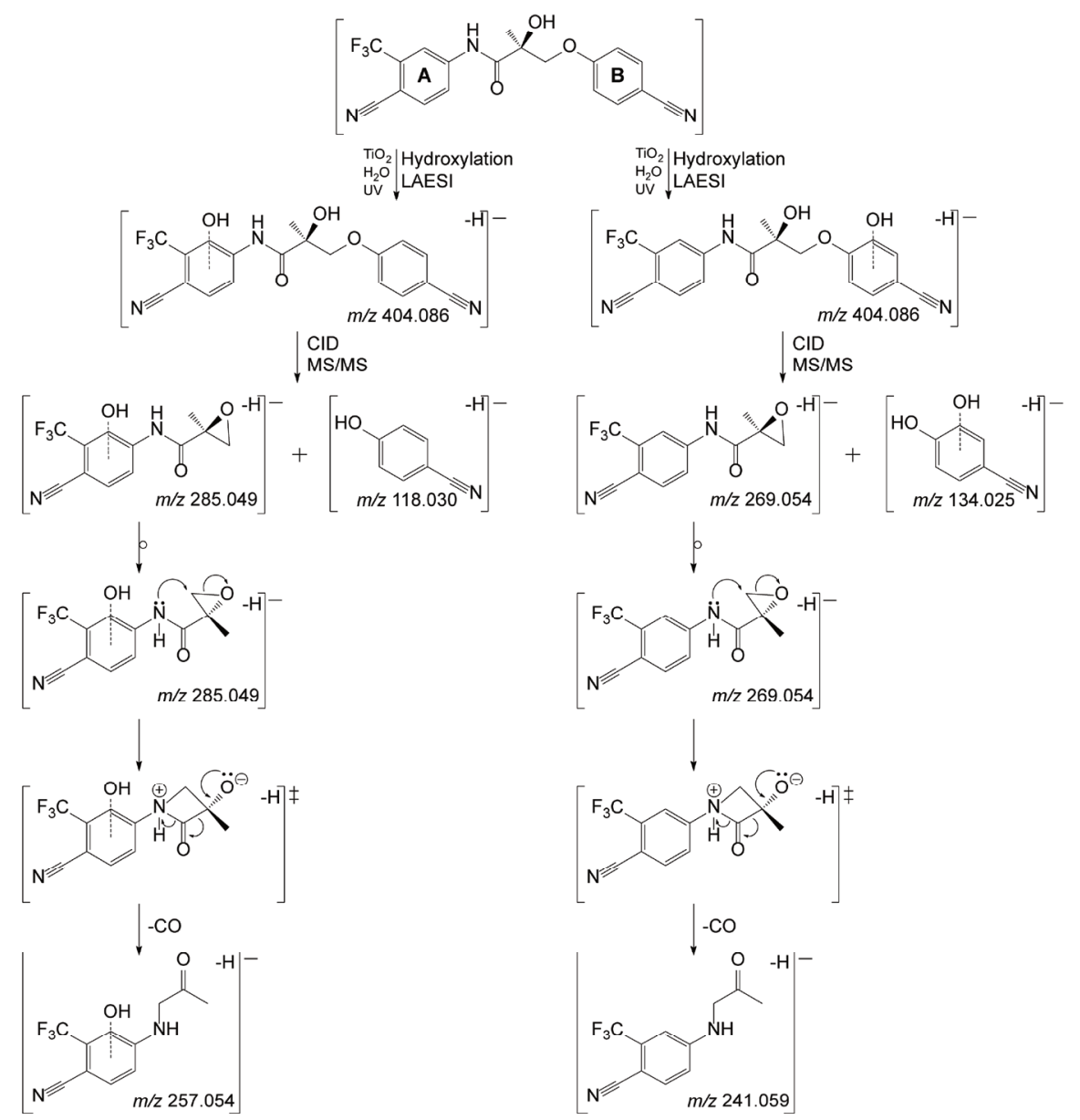

Figure S7. MS/MS on ostarine hydroxylation product ions with proposed structures. We proposed an alternative fragmentation mechanism to form $\mathrm{m} / \mathrm{z} 257$ (similarly 241) than which has been proposed previously. ${ }^{7}$ 


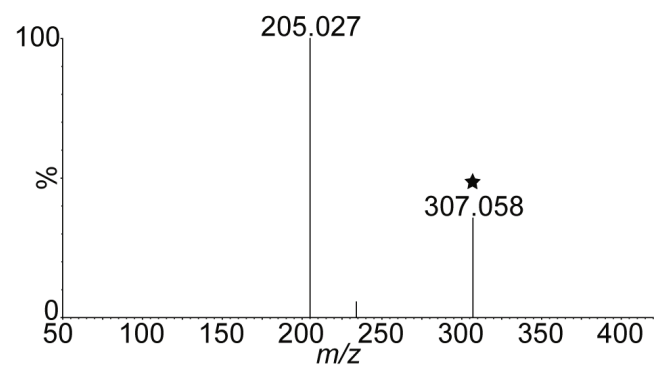

Figure S8. Andarine MS/MS on $\mathrm{m} / \mathrm{z} 307$. Selected precursor ion is annotated with a star.

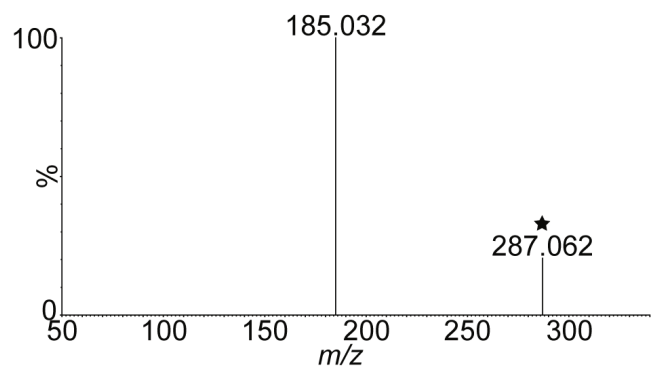

Figure S9. Ostarine MS/MS on $\mathrm{m} / \mathrm{z} 287$. Selected precursor ion is annotated with a star.
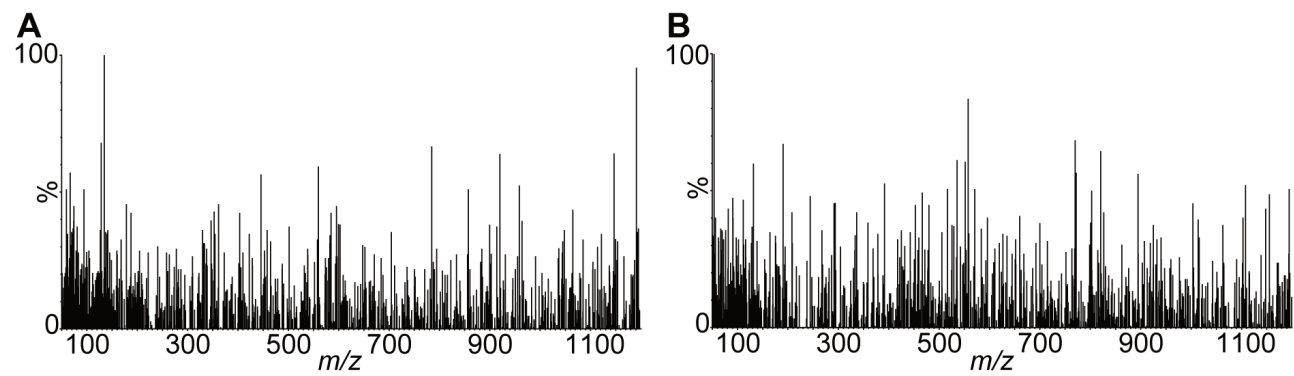

Figure S10. Background subtracted mass spectra of Ostarine and Andarine on glass slides without $\mathrm{TiO}_{2}$ coating. Both samples - Figures A (Ostarine) and B (Andarine) - were treated as described in the experimental section. This experiment was meant to detect any effect of photodegradation as a result of uncatalyzed UV exposure; yet, no effect (product) was observed. 
Chapter 4

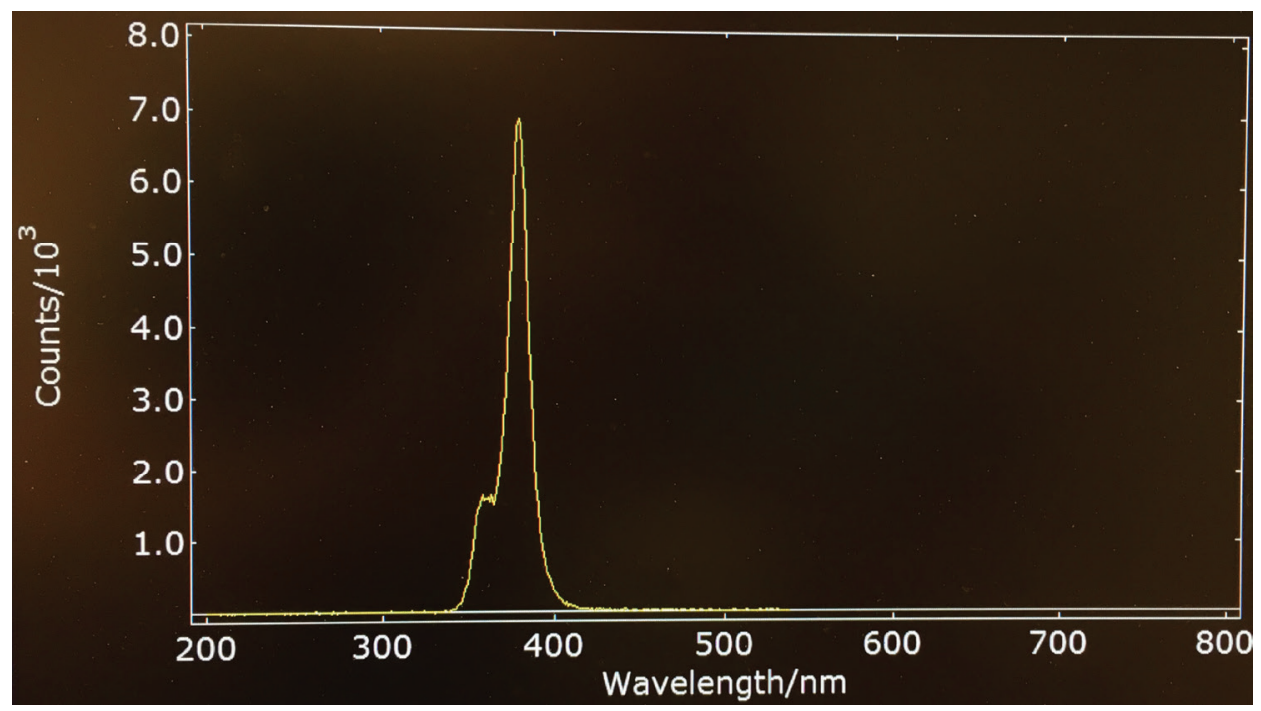

Figure S11. Emission spectrum of used UV lamp. 


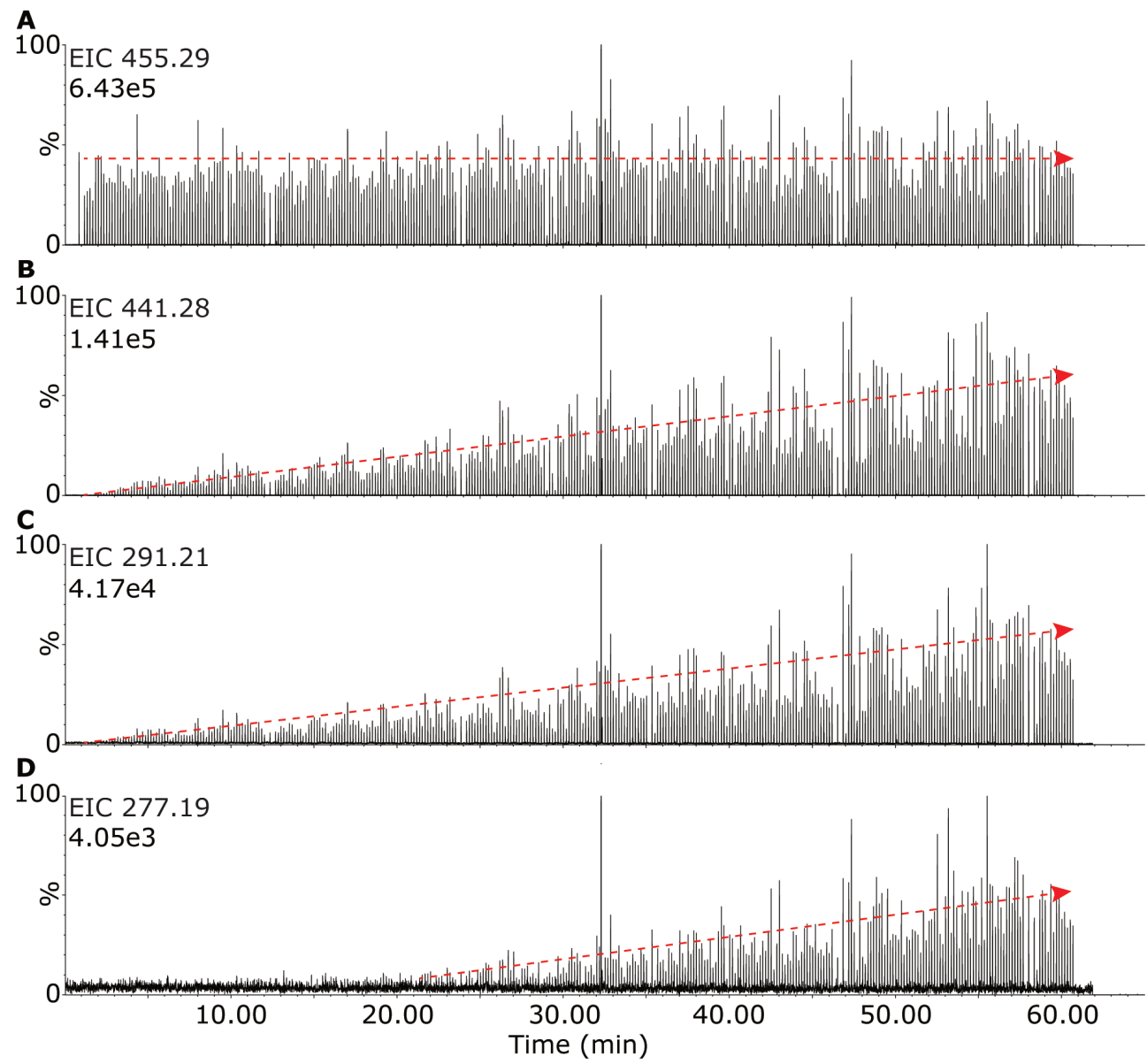

Figure S12. EIC's of verapamil and oxidation products generated by time-resolved photocatalysis LAESI-MS using suspended $\mathrm{TiO}_{2}$ nanoparticles. Each peak in the EIC's is a result of a laser pulse (injection) with a frequency of $0.1 \mathrm{~Hz}$. The red lines indicate the trend in the raw data. A shows EIC of verapamil $[\mathrm{M}+\mathrm{H}]^{+}$which shows a constant signal (apart from normal instrumental - LAESI - variation) for 60 minutes reaction time. $\mathbf{B}$ and $\mathbf{C}$ show the $\mathrm{TiO}_{2}$ photocatalysis products $\left[\mathrm{M}-\mathrm{CH}_{2}+\mathrm{H}\right]^{+}$(demethylation) and $[\mathrm{M}-164+\mathrm{H}]^{+}(\mathrm{N}$-dealkylation), respectively. Finally, $\mathbf{D}$ shows the product of both the $\mathrm{N}$-dealkylation and demethylation, which are depicted in $\mathbf{B}$ and $\mathbf{C}$, and is observed starting from 25 minutes onwards. This result shows consecutive reactions of already formed products and demonstrates the relevance of time-resolved measurements. 
Chapter 4

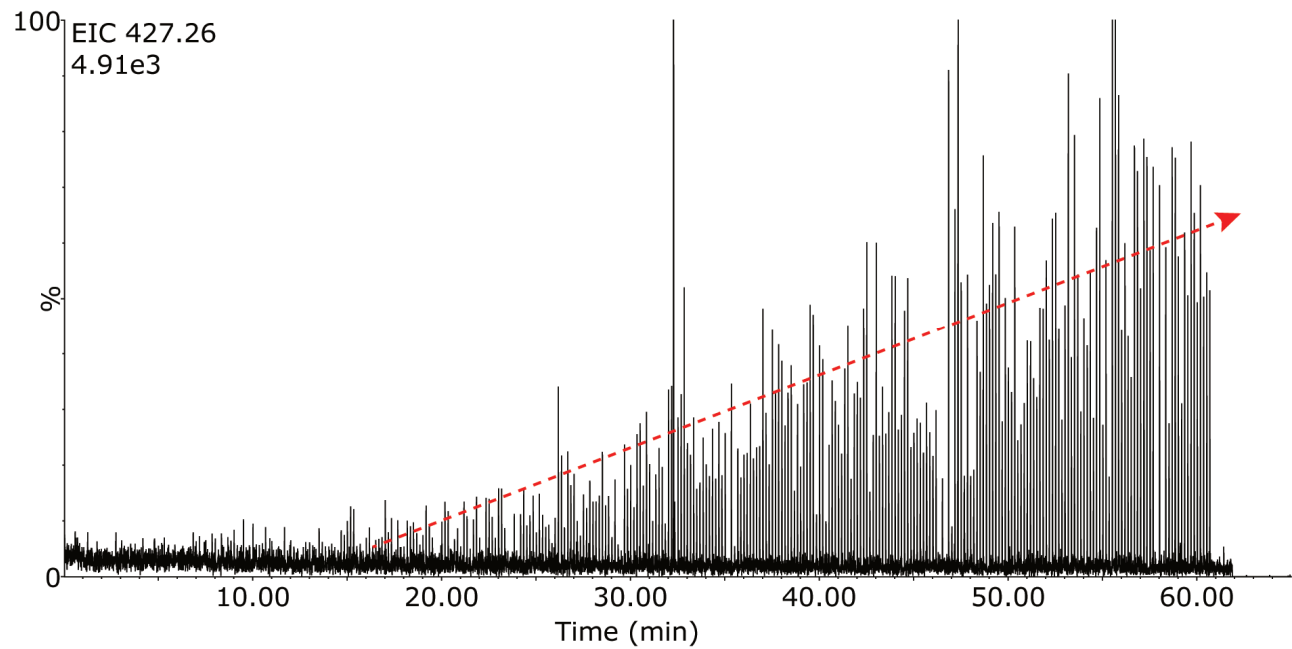

Figure S13. EIC of $m / z 427.26$ following time-resolved $\mathrm{TiO}_{2}$ photocatalyzed oxidation LAESI-MS of verapamil. Each peak in the EIC is a result of a laser pulse (injection) with a frequency of $0.1 \mathrm{~Hz}$. The red line indicates the trend in the raw data. 

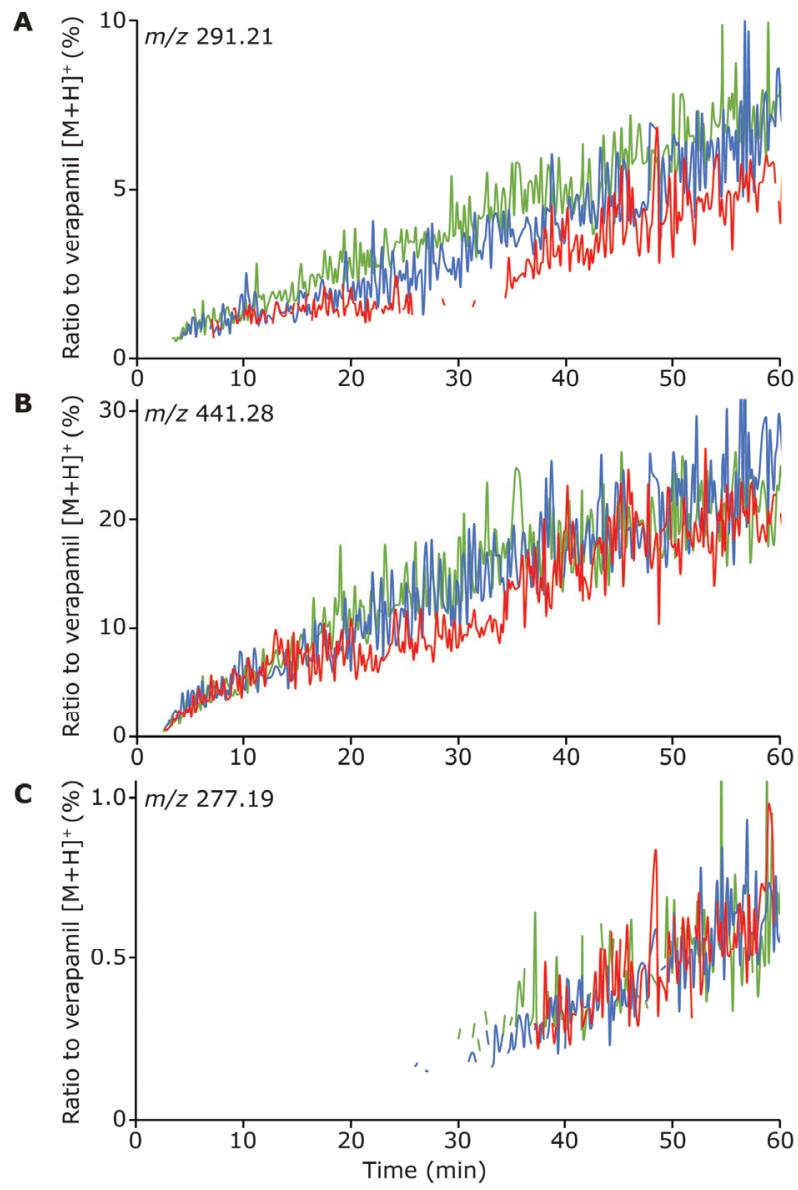

Figure S14. Individual data for oxidation products generated by time-resolved $\mathrm{TiO}_{2}$ photocatalysis $\mathrm{LAESI-MS}$ as presented in Figure 3. Each signal is normalized to verapamil $[\mathrm{M}+\mathrm{H}]^{+}$. A shows data for $\mathrm{m} / z 291.21$ ( $\mathrm{N}$-dealkylation). B presents data for $m / z 441.28$ (demethylation) and C depicts data for $m / z 277.19$ (both the $\mathrm{N}$-dealkylation and demethylation).

\section{References}

1 Walles, M.; Thum, T.; Levsen, K.; Borlak, J. J. Chromatogr. A 2002, 970, 117-130.

2 Ruokolainen, M.; Miikkulainen, V.; Ritala, M., et al. Anal. Chem. 2017, 89, 1121411218.

3 Calza, P.; Pazzi, M.; Medana, C., et al. J. Pharm. Biomed. Anal. 2004, 35, 9-19.

4 Zhu, M.; Zhao, W.; Jimenez, H., et al. Drug Metab. Dispos. 2005, 33, 500-507.

5 Thevis, M.; Beuck, S.; Höppner, S., et al. J. Am. Soc. Mass Spectrom. 2012, 23, 537-546.

6 Williams, T. M.; Kind, A. J.; Houghton, E.; Hill, D. W. J. Mass Spectrom. 1999, 34, 206-216.

7 Thevis, M.; Thomas, A.; Fußhöller, G., et al. Rapid Commun. Mass Spectrom. 2010, $24,2245-2254$. 


\section{Chapter 5}

\section{Laser Ablation Electrospray Ionization Hydrogen/Deuterium Exchange for Structure Elucidation in Ambient Mass Spectrometry Imaging}

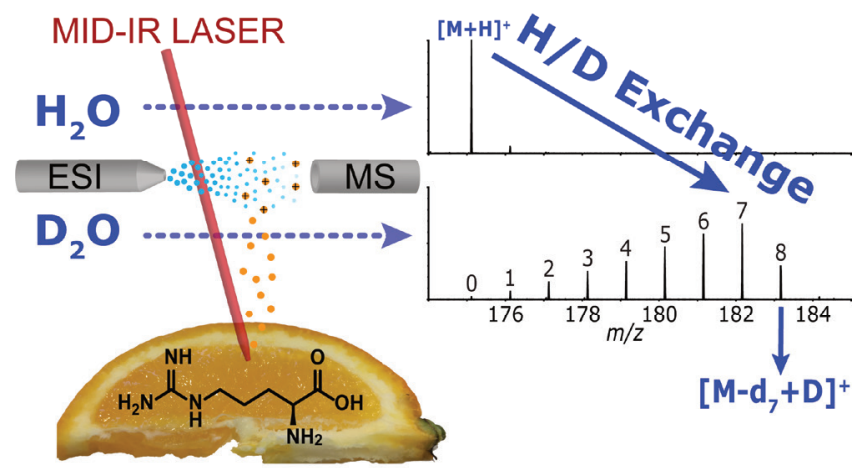

This Chapter has been submitted as:

Fred A. M. G. van Geenen, Frank W. Claassen, Maurice C. R. Franssen, Han Zuilhof and Michel W. F. Nielen. Laser Ablation Electrospray Ionization Hydrogen/Deuterium Exchange for Structure Elucidation in Ambient Mass Spectrometry Imaging. 


\section{Abstract}

Ambient mass spectrometry (MS) currently draws great interest for its simple and fast analysis capabilities and its feasibility in imaging approaches. Identification and confirmation of known as well as unknown (bio)chemical entities in ambient MS mostly involves accurate mass determination, often in combination with MS/MS or $\mathrm{MS}^{n}$ work flows. However, an accurate mass only provides the elemental composition of the (bio)molecule, still resulting in numerous possible structures. MS/MS procedures are often insufficient in differentiating between the hundreds possible candidate substances in database searches. Obtaining additional information and thereby improving structural assignment as well as reducing the vast number of possible candidates is thus of paramount importance in any ambient MS study to compensate for the lack of extensive sample preparation or chromatographic separation. Here we present an ambient hydrogen/deuterium exchange (HDX) laser ablation electrospray ionization (LAESI) MS method for structure elucidation and confirmation of (bio)molecules. The concept was demonstrated with both small molecules such as melamine and monosaccharides as well as large molecules such as peptides and proteins. Moreover, the same approach could be applied to mass spectrometry imaging (MSI) as shown by the ambient MSI of arginine and oligosaccharides on an orange slice and an ubiquitin spiked muscle tissue sample. Eventually, this approach will allow spatially resolved $\mathrm{MSI}$ of different protein conformers and may have a major impact in the life sciences. 


\section{Introduction}

Mass spectrometry (MS) studies commonly aim to find the identity and/or quantity of molecules in a sample. In mass spectrometry imaging (MSI) studies the localization of molecules is also included to find, e.g., lipid profiles, the accumulations of drugs and their metabolites, and peptide or protein distributions in tissue. ${ }^{1-5}$ However, often samples require laborious pretreatment steps and/or vacuum conditions to be compatible with MS(I) analysis. Sample pretreatment prevents real-time analysis and can largely affect the outcome of MS analysis, such as analyte losses and delocalization of molecules. ${ }^{6,7}$ Additionally, samples can be disrupted or damaged when affected by vacuum conditions. ${ }^{8,9}$ Ambient MS was therefore introduced to record mass spectra of samples in their native environment, without any sample pretreatment. ${ }^{10-13}$

Structural identification of (bio)molecular entities in ambient MS(I) is of prime interest and largely depending on accurate mass measurements, usually combined with MS/MS or $\mathrm{MS}^{\mathrm{n}}$ approaches. ${ }^{14}$ The accurate mass is acquired with high resolution instruments such as time-of-flight and orbitrap MS, and provides the elemental composition of the (bio)molecule. ${ }^{15}$ This elemental composition usually still yields numerous candidates of chemical substances. Databases can be used to obtain known and/or expected molecular structures comprising the same elemental composition. ${ }^{16} \mathrm{MS} / \mathrm{MS}$ approaches can then further elucidate the (bio)molecular structure but with hundreds of molecular structure options, this would require the availability of large libraries of standard substances. ${ }^{17}$ Also orthogonal methods like ion mobility (IMS) or online reactions can be used during ambient MS(I) data acquisition to obtain additional information, but these have limited success for elucidation of unknown entities and are mostly used for improved selectivity and signal-to-noise ratio. ${ }^{18-21}$ The development of methods to obtain additional molecular structural information is thus of utmost importance for reducing the number of options and thereby increasing the certainty in, as well as the speed of, identification in ambient MS(I).

One such an approach which may assist in the identification of unknown entities is hydrogen/deuterium exchange (HDX) MS. ${ }^{22}$ HDX-MS is a method to obtain the number of exchangeable hydrogens in a molecule, present in, e.g., $-\mathrm{NH},-\mathrm{OH}$ and $-\mathrm{SH}$ moieties. The $\mathrm{m} / \mathrm{z}$ value of the unknown entity will increase accordingly with each included deuteron, providing valuable information on the number of exchangeable protons. This information can then be used to assist in (bio)molecular structure datamining, as a method to probe protein $3 \mathrm{D}$ structure, and/or to investigate molecular complex formation. ${ }^{23-25}$ Common HDX procedures are focused on adding a deuterated gas to trapped gas-phase ions for exchange reactions inside the vacuum of the mass spectrometer, ${ }^{26}$ using a deuterated reaction gas in chemical ionization $(\mathrm{Cl}),{ }^{27}$ or by dissolving/diluting the sample in a deuterated solvent prior to MS analysis. ${ }^{28}$ However, gas-phase HDX of trapped ions is nearly impossible to combine with the - wide $m / z$ range - high ion throughput of non-targeted approaches that are common in 
imaging and -omics studies. $\mathrm{Cl}$, or desorption atmospheric pressure chemical ionization (DAPCI), is only compatible with volatiles and very low molecular weight molecules, limiting its use for surface and tissue analysis. ${ }^{29}$ Furthermore, dissolving/diluting samples obviously causes delocalization of analytes, is incompatible with surface analysis, and may also suffer from back-exchange. A combination of HDX with ambient MS(I) would be a valuable addition for structure identification in surface analysis including tissue. Apart from an initial DART setup for ambient gas-phase HDX, ambient MS has not been explored for HDX yet. ${ }^{30}$

Laser ablation electrospray ionization (LAESI) is an ambient MS ionization technique that can also be used for imaging. ${ }^{31}$ LAESI-MS applicability was demonstrated in the analysis of tissue, ${ }^{32-36}$ food contaminants, ${ }^{37}$ synthetic materials, ${ }^{38}$ and single cells. ${ }^{39-41}$ Furthermore, LAESI-MS was proven viable for top-down MS of intact proteins, ${ }^{42}$ and for performing and/or monitoring online time-resolved reactions. ${ }^{20,43,44}$ In LAESI-MS analysis, ablated sample material is continuously extracted by charged microdroplets that are produced by an orthogonally placed electrospray emitter, prior to MS analysis. In an HDX-LAESI-MS approach the ESI solvent can easily be replaced with a deuterated one, resulting in a simple ambient MS HDX method that would also enable imaging. As shown in previous ESI studies, the problem of back-exchange would be minimal as a result of the continuous ESI spray which is directed towards the MS inlet. ${ }^{45-47}$ Obviously different deuterium donors might be exploited, although in the present work $\mathrm{D}_{2} \mathrm{O}$ was used aiming for, amongst others, native protein conditions. HDX reaction kinetics of exchangeable hydrogens is expected to be rapid due to the liquid microdroplet environment, yielding up to complete conversions for at least small molecules. ${ }^{48}$

In this research we report a novel ambient MS approach, viz. HDX-LAESI, for (bio)molecular structure elucidation and confirmation. This method was demonstrated with the HDX reactions of small molecules, a peptide and two proteins, and its performance was critically compared with current literature. In addition, the applicability in (bio)molecular structure identification and MSI was shown in combination with ion mobility for biomolecules like arginine and oligosaccharides, detected directly from an orange slice. Finally, the feasibility of ambient HDX-LAESI-MS(I) in native protein analysis was initially demonstrated with a ubiquitin spiked muscle tissue sample. 


\section{Experimental section}

\section{Materials}

Ultrapure water $\left(\mathrm{H}_{2} \mathrm{O}\right) 18.2 \mathrm{M} \Omega \times \mathrm{cm}^{-1}$ at $25^{\circ} \mathrm{C}$ was freshly produced with a Millipore (Molsheim, France) Integral 3 system. Deuterium oxide $\left(\mathrm{D}_{2} \mathrm{O}\right)$ 99.9\% atom $\mathrm{D}$, melamine, bradykinin, a-lactalbumin from bovine milk, ubiquitin from bovine erythrocytes, deuterated formic acid (FA-d 2$) 99 \%+$ atom D, ammonium acetate $\left(\mathrm{NH}_{4} \mathrm{Ac}\right)$ LC-MS grade, acetic acid (HAc) LC-MS grade, and ammonium hydroxide $\left(\mathrm{NH}_{4} \mathrm{OH}\right)$ LC-MS grade were purchased from Sigma-Aldrich (Zwijndrecht, The Netherlands). A new ampule of $\mathrm{D}_{2} \mathrm{O}$ was used for each analysis. An orange and chicken muscle tissue were obtained from a local supermarket.

\section{Ambient HDX-LAESI-MS}

A Protea Biosciences (Morgantown, WV) LAESI DP-1000 system was coupled to a Waters (Manchester, U.K.) Synapt G2-S traveling wave ion mobility (TWIM) time of flight mass spectrometer (TOFMS), and used for all analyses. Masslynx v4.1 SCN 883 (Waters) was used to control the experimental settings of the Synapt G2-S, which was operated in positive ion TOFMS resolution mode (mass resolution approximately $18000 \mathrm{FWHM}$ ) with a scan time of 1 $\mathrm{s}$, source temperature $150{ }^{\circ} \mathrm{C}$, sample cone $40 \mathrm{~V}$, and source offset $80 \mathrm{~V}$. In the case of oligosaccharide analysis, TWIM was applied using the following experimental conditions: the IMS wave velocity was set at $1200 \mathrm{~m} / \mathrm{s}$ and wave height was $40 \mathrm{~V}$. Driftscope v2.7 (Waters) was used to individually select drift time regions corresponding to oligosaccharides (Figure $\mathrm{S} 1)$. Selected drift time data - containing all $\mathrm{m} / \mathrm{z}$ information residing within the selection were exported back into Masslynx and background-subtracted. In the case of native protein analysis experiments the source offset was $150 \mathrm{~V}$ and quad profile was set to manual fixed at $500 \mathrm{Da}$. The LAESI DP-1000 system was equipped with a $2940 \mathrm{~nm}$ mid-IR laser and controlled by LAESI desktop software v.2.0.1.3 (Protea Biosciences). Sample solutions were put in a 96-well plate. At each well 35 laser pulses were used at a frequency of $5 \mathrm{~Hz}$ to produce a plume of ablated sample material, which was continuously extracted with charged microdroplets generated from an orthogonally placed electrospray emitter prior to MS analysis. The electrospray solvent was either $\mathrm{H}_{2} \mathrm{O}$ or $\mathrm{D}_{2} \mathrm{O}$ at a flowrate of $3 \mu \mathrm{L} / \mathrm{min}$. In case of protein analysis $10 \mathrm{mM} \mathrm{NH}_{4} \mathrm{Ac}(\mathrm{pH}$ 7) was added to ensure native conditions. Electrospray voltage was set at $\sim 4 \mathrm{kV}$ in order to have a stable signal. Background-subtracted mass spectra were generated using the "combine spectrum" function in Masslynx: 5 scans were combined and 20 scans of electrospray background were subtracted.

\section{Ambient HDX-LAESI-MSI of Orange and Tissue Slices}

An orange was sliced with a kitchen knife and placed - on a glass slide - onto the temperature controlled sample stage maintained at $4{ }^{\circ} \mathrm{C}$. (HDX-)LAESI-MS data were 
acquired from a $14 \times 17$ pattern (238 sample locations) in a sampling area defined from an optical image. The chicken muscle tissue was sliced with a scalpel and spiked with 50 nanomoles ubiquitin, and dried in ambient air for $10 \mathrm{~min}$. The sample stage temperature was set at $4{ }^{\circ} \mathrm{C}$ and data were acquired from a $12 \times 10$ pattern (120 sample locations). For both analyses 20 laser pulses were used at each location with a frequency of $10 \mathrm{~Hz}$ and $3 \mathrm{~s}$ dwell time per analyses location. The laser spot size was approximately $200 \mu \mathrm{m}$ and the in-between spot interval was $1 \mathrm{~mm}$. These experimental settings resulted in total analysis times of 43 and $18 \mathrm{~min}$, respectively. Proteaplot v2.0.8.5 (Protea Biosciences) was used to create maximum intensity ion maps.

\section{Results and Discussion}

\section{Structure Elucidation of Small Molecules by Ambient HDX-LAESI-MS}

The feasibility of ambient HDX-LAESI-MS as a tool for structure elucidation was first investigated with the model compounds melamine, comprising multiple - $\mathrm{NH}$ groups, and monosaccharides, containing multiple $-\mathrm{OH}$ groups. Melamine has an elemental composition of $\mathrm{C}_{3} \mathrm{~N}_{6} \mathrm{H}_{6}$ and holds 6 exchangeable $-\mathrm{NH}_{2}$ protons (chemical structure is included in Figure $1 A$ ), this should experimentally result in a maximum $m / z$ increase of 7 for $\left[M-d_{6}+D\right]^{+}$. Figure $1 \mathrm{~A}-\mathrm{B}$ presents LAESI-MS background-subtracted mass spectra of a $100 \mu \mathrm{M}$ aqueous melamine solution with $\mathrm{H}_{2} \mathrm{O}$ (Fig. 1A) and $\mathrm{D}_{2} \mathrm{O}$ (Fig. 1B) as ESI solvents. While using $\mathrm{H}_{2} \mathrm{O}$ as ESI solvent an $[\mathrm{M}+\mathrm{H}]^{+}$ion was detected at $m / z$ 127.08. However, when changing to $\mathrm{D}_{2} \mathrm{O}$ a $\mathrm{m} / \mathrm{z}$ distribution ranging from 128.08 to 134.12 was observed. These values correspond to protonated melamine $\left[\mathrm{M}-\mathrm{d}_{0}+\mathrm{H}\right]^{+}$and stepwise increase the deuterium content up to a completely hydrogen/deuterium $(H / D)$ exchanged value of $m / z 134.12$ for $\left[M-d_{6}+D\right]^{+}$, which nicely corresponds with previously reported liquid and gas phase melamine HDX studies. ${ }^{30,49}$ The millisecond reaction in electrospray microdroplets upon LAESI provides sufficient time for $H / D$ exchange up to completion. Following elemental composition assessment based on accurate mass measurement, a SciFinder ${ }^{\circledR}$ elemental composition search was performed to obtain structural isomers options. $\mathrm{C}_{3} \mathrm{~N}_{6} \mathrm{H}_{6}$, initially resulted in 25 isomers, however, when only structures comprising six proton exchange sites were included, the number of structures was substantially reduced to only five.

The most common $\mathrm{C}_{6}$ monosaccharides have an elemental composition of $\mathrm{C}_{6} \mathrm{H}_{12} \mathrm{O}_{6}$ and contain five exchangeable $-\mathrm{OH}$ protons (chemical structure of a cationized monosaccharide, D-fructose, is included in Figure 1C). In positive ESI mode such saccharides are cationized and commonly detected as sodium adducts. ${ }^{50,51}$ As a consequence HDX is expected to result in a maximum $\mathrm{m} / \mathrm{z}$ shift of $+5 \mathrm{Da}$. Figure $1 \mathrm{C}$-D depicts LAESI-MS (with $\mathrm{H}_{2} \mathrm{O}$ as ESI solvent) and HDX-LAESI-MS (with $\mathrm{D}_{2} \mathrm{O}$ as ESI solvent) background-subtracted mass spectra of $\mathrm{C}_{6}$ monosaccharides in $20 \times$ diluted orange juice. With LAESI-MS a single $\mathrm{m} / \mathrm{z}$ value is detected at 203.05, corresponding to cationized monosaccharides $[\mathrm{M}+\mathrm{Na}]^{+}$. HDX- 
LAESI-MS however shows a clear increasing isotope pattern ranging from $\mathrm{m} / \mathrm{z} 203.05$ for [M$\left.\mathrm{d}_{0}+\mathrm{Na}\right]^{+}$up to a fully $\mathrm{H} / \mathrm{D}$ exchanged $m / z 208.08$ for $\left[\mathrm{M}-\mathrm{d}_{5}+\mathrm{Na}\right]^{+}$. This is in line with the number of exchanges for the previously observed $\left[\mathrm{M}-\mathrm{d}_{5}+\mathrm{D}\right]^{+}$monosaccharide ion in an atmospheric pressure chemical ionization study when $\mathrm{D}_{2} \mathrm{O}$ was used as a solvent. ${ }^{52} \mathrm{~A}$ SciFinder ${ }^{\circledR}$ elemental composition search was performed (on $\mathrm{C}_{6} \mathrm{H}_{12} \mathrm{O}_{6}$ ) and 450 results were obtained including all stereoisomers. 95 of the obtained isomers could be eliminated following the selection of five proton exchange sites. Both melamine and monosaccharides demonstrate that ambient HDXLAESI-MS is a simple and expeditious technique for a significant simplification of the structure elucidation for molecules comprising $\mathrm{N}-\mathrm{H}$ or $\mathrm{O}-\mathrm{H}$ bonds, reducing the number of SciFinder ${ }^{\circledR}$ candidates substantially by $80 \%$ and $21 \%$, respectively.
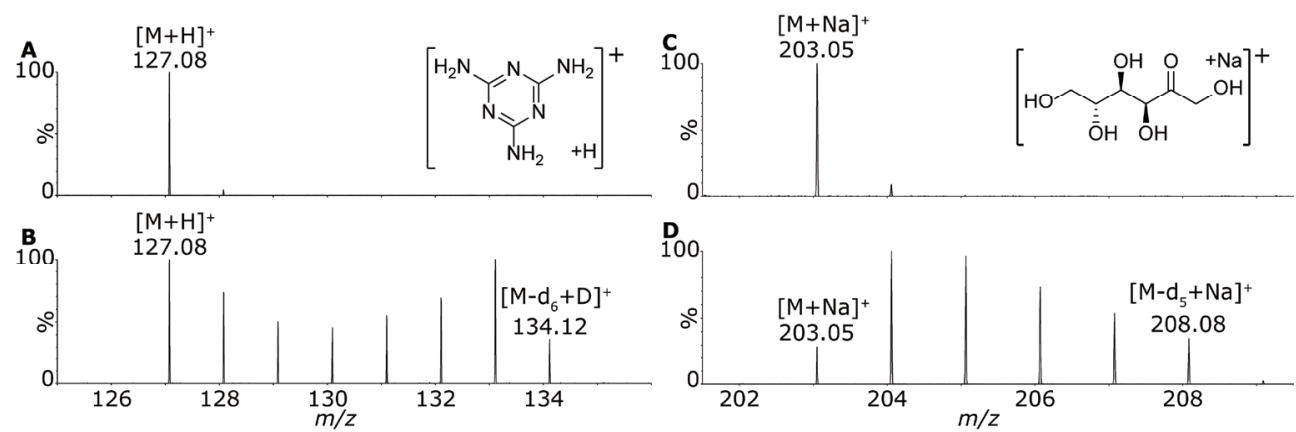

Figure 1. LAESI-MS and HDX-LAESI-MS of melamine and monosaccharides. A and $\mathbf{B}$ present LAESI-MS (ESI solvent was $\mathrm{H}_{2} \mathrm{O}$ ) and HDX-LAESI-MS (ESI solvent was $\mathrm{D}_{2} \mathrm{O}$ ) of an aqueous melamine solution, respectively. A also shows the chemical structure of protonated melamine. $\mathbf{C}$ and $\mathbf{D}$ show LAESI-MS and HDX-LAESI-MS of six-carbon monosaccharides in $20 \times$ diluted (with $\mathrm{H}_{2} \mathrm{O}$ ) orange juice, respectively. $\mathbf{C}$ additionally shows the structure of the most abundant (cationized) monosaccharide (D-fructose) in oranges. ${ }^{53}$

\section{Ambient HDX-LAESI-MS of Peptides}

Besides amine and hydroxyl groups HDX is commonly performed to exchange amide bond protons located in peptide and protein backbones. ${ }^{25,54,55} \mathrm{~A}$ nonapeptide, bradykinin (ArgPro-Pro-Gly-Phe-Ser-Pro-Phe-Arg, Figure S2), was examined as model compound. The HDX rate of freely exposed amide hydrogens is dependent on $\mathrm{pH}$, temperature, and solvent, due to the amphiprotic properties of the amide group. ${ }^{56,57}$ The exchange of amide hydrogen atoms at room temperature has a minimum rate at approximately $\mathrm{pH} 3$, with increasing rates by base or acid catalysis on either side of this minimum. ${ }^{58,59}$ Electrospray-generated charged microdroplets have decreasing $\mathrm{pH}$ values along the spray axis towards the MS inlet. ${ }^{60}$ Since the laser-ablated sample aerosol is continuously extracted by these charged microdroplets, the electrospray solvent $\mathrm{pH}$ is an important parameter in HDX-LAESI-MS analysis of bradykinin. Figure $\mathrm{S} 3$ shows mass spectra of bradykinin obtained with $0.1 \% \mathrm{FA}-\mathrm{d}_{2}$ or $10 \mathrm{mM}$ $\mathrm{NH}_{4} \mathrm{Ac}$ at either $\mathrm{pH} 7$ or $\mathrm{pH} 9$ as electrospray additives. When FA-d2 was added to the ESI 
solvent $\left(D_{2} \mathrm{O}\right)$ almost no $H / D$ exchanges were detected, whereas when $\mathrm{NH}_{4} A c$ was added at $\mathrm{pH} 7$ or 9 approximately 14 exchanges were observed. Indeed addition of FA-d2 (pH ca. 3) inhibits the HDX rate, thus an aqueous ESI solvent containing $10 \mathrm{mM} \mathrm{NH}_{4} \mathrm{Ac}$ at $\mathrm{pH} 7$ was used for peptide and protein samples providing rapid HDX and native conditions.

Figure 2 shows LAESI-MS and HDX-LAESI-MS background-subtracted mass spectra of double charged bradykinin ions (single charged ions are presented in Figure S4). The deuterium isotope pattern observed following HDX was similar for both single and double charged ions ranging from 0 to approximately 14 or 15 exchanges (Figures 2B and S4B), likely with some overlap of $H / D$ exchanges and ${ }^{13} \mathrm{C}$ isotopes ranging from $M+1$ to $M+18$. Bradykinin includes 17 exchangeable protons, thus a maximum of $18-$ for $\left[M-d_{17+} D\right]^{+}-$or $19-$ for $[M-$ $\left.\mathrm{d}_{17+2 D}\right]^{2+}-$ exchanges could be observed. Instead, the HDX-LAESI-MS results show up to 14 or 15 exchanges instead of the maximum of 18 or 19 , this is likely due to a reduced reaction rate for some exchangeable hydrogens as a result of intramolecular hydrogen bonding. ${ }^{61,62}$ Dual ESI microdroplet fusion experiments reported by the Zare group showed an increasing number of $\mathrm{H} / \mathrm{D}$ exchanges in bradykinin with an increased distance between the droplet fusion center (of $\mathrm{H}_{2} \mathrm{O}$ droplets containing $1 \mu \mathrm{M}$ ubiquitin and $\mathrm{D}_{2} \mathrm{O}$ droplets as the $2^{\text {nd }} \mathrm{ESI}$ spray) and the mass spectrometer inlet, i.e. with an increased reaction time. ${ }^{63}$ In their experiments, approximately 10 exchanges were reported for a distance of $\sim 2 \mathrm{~mm}$. The current LAESI setup, although a different technique, involves similar microdroplet chemistry and comprises a $5 \mathrm{~mm}$ reaction path, which we interpret to yield an increased reaction time as well. The obtained HDX-LAESI-MS results are in line with the expectations for a longer distance. ${ }^{63}$ In contrast to solvent-based HDX, gas-phase HDX studies of isolated bradykinin ions in trap based mass spectrometers have shown a substantial difference - even over a factor 1000 in relative $\mathrm{HDX}$ rate between $[\mathrm{M}+\mathrm{H}]^{+}$and $[\mathrm{M}+2 \mathrm{H}]^{2+}$ ions when studied up to $80 \mathrm{~s} .{ }^{64,65}$ In the current HDX-LAESI-MS setup similar isotope patterns were detected for both single and double charged bradykinin ions. This clearly suggests that with LAESI-MS the HDX reaction does not occur in the gas phase.

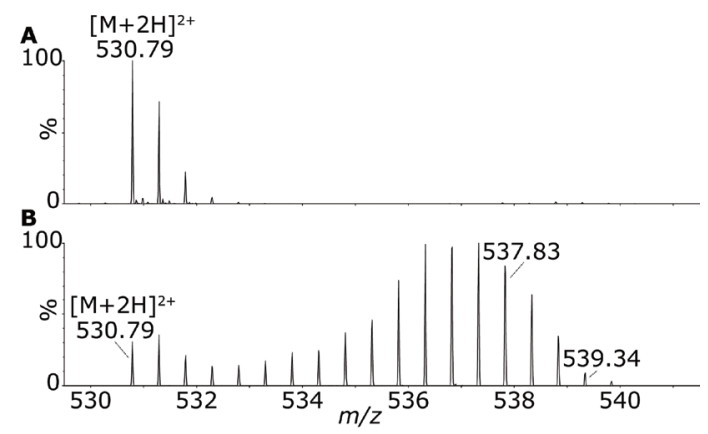

Figure 2. LAESI-MS and HDX-LAESI-MS of bradykinin $\left(0.2 \mathrm{mM}\right.$ in $\left.\mathrm{H}_{2} \mathrm{O}\right)$. A presents LAESI-MS (ESI solvent was $\mathrm{H}_{2} \mathrm{O}+$ $10 \mathrm{mM} \mathrm{NH}_{4} \mathrm{Ac} \mathrm{pH}$ 7) and $\mathbf{B}$ presents HDX-LAESI-MS (ESI solvent was $\mathrm{D}_{2} \mathrm{O}+10 \mathrm{mM} \mathrm{NH}_{4} \mathrm{Ac} \mathrm{pH}$ 7). 


\section{Feasibility of Ambient HDX-LAESI-MS to Support Native Protein Conformation Analysis}

In case of large molecules such as proteins HDX kinetics are also largely influenced by effects originating from their 3D structure(s), like, e.g., intramolecular hydrogen bonding and the - HDX site - distance to the protein surface..$^{66,67}$ These effects on exchange reaction rates are used to obtain information on protein 3D conformations. ${ }^{24}$ In top-down proteomics conformational information is obtained following time-resolved HDX with subsequent electron transfer dissociation (ETD) or electron capture dissociation (ECD) MS/MS experiments. ${ }^{68}$ Fragments originally located at the outside of the 3D structure then display a different amount of deuterium incorporation in comparison with fragments originally located in the core of the 3D structure. ${ }^{69,70}$ Here, two proteins, ubiquitin and $\alpha$-lactalbumin, were used to investigate whether HDX-LAESI-MS provides deuterium incorporation in native protein analysis and, if so, whether ambient HDX-MSI of proteins would be feasible. Ubiquitin is a $8.6 \mathrm{kDa}$ protein that contains 76 amino acid residues, comprises 144 exchangeable hydrogen atoms, and plays a key role in the regulation of biological protein degradation. ${ }^{46,71}$ Figure $3 \mathrm{~A}-\mathrm{C}$ shows the background-subtracted mass spectra of the 10+ charge state of ubiquitin obtained with LAESIMS (Fig. 3A) and HDX-LAESI-MS (Fig. 3B) of a ubiquitin solution, and HDX-LAESI-MS following prolonged ubiquitin incubation in $95 \% \mathrm{D}_{2} \mathrm{O}$ (Fig. $3 \mathrm{C}$ ). Comparing LAESI-MS with HDX-LAESI-MS a mass shift for the isotope distribution of the ubiquitin $10+$ ion of approximately 12 to 60 Da was observed. However, when ubiquitin was dissolved in $95 \% D_{2} \mathrm{O}$ and incubated for 30 min prior to HDX-LAESI-MS analysis, a mass shift of ca. 75 to $125 \mathrm{Da}$ was detected. These spectra evidently demonstrate a time-resolved effect on the number of deuterium incorporations. Previously an ESI-MS study found a mass shift of approximately 80 Da after 50 ms reaction time for denatured ubiquitin. ${ }^{72}$ With the millisecond reaction time and native protein analysis conditions as used here the experimentally observed average mass shift of $36 \mathrm{Da}$ would be in accordance with expectations. In future protein studies and using a more dedicated protein MS/MS instrument, precursor ion(s) in the isotope distribution could be selected for fragmentation studies by ETD or ECD tandem MS for more detailed protein conformation assessment.

Analogously, bovine $\alpha$-lactalbumin is a $14 \mathrm{kDa}$ protein and contains 123 amino acid residues, comprises 232 exchangeable protons, and regulates the production of lactose in milk..$^{73,74}$ The same set of experiments as with ubiquitin was performed on $\alpha$-lactalbumin and Figure 3D-E show background-subtracted mass spectra of the 7+ $\alpha$-lactalbumin ion. Notably 3 additional signals, as a result of $\mathrm{Na}^{+}$and/or $\mathrm{Ca}^{2+}$ cationization, were detected with LAESIMS analysis (Fig. 3D). Therefore the approximate mass shift between conventional LAESIMS and HDX-LAESI-MS as well as HDX-LAESI-MS of a lactalbumin solution following prolonged incubation in $\mathrm{D}_{2} \mathrm{O}(95 \%)$ is complicated here due to significant overlap with $\mathrm{Na}^{+}$and $\mathrm{Ca}^{2+}$ adducts (Figure 3D-E). These adducts broaden and extend the protein isotope distribution even beyond the expected $m / z$ value of a fully $H / D$ exchanged $\alpha$-lactalbumin at approximately $\mathrm{m} / \mathrm{z} 2060$. It is unlikely that $\mathrm{m} / \mathrm{z} 2060$ would be obtained with $\alpha$-lactalbumin 
incubated in $\mathrm{D}_{2} \mathrm{O}$ for 30 min since a period of at least 3 days was required for complete HDX of a-lactalbumin in a Fourier-transform infrared (FTIR) and nuclear magnetic resonance (NMR) spectroscopy study. ${ }^{75}$ Nonetheless, for both proteins studied clear differences in mass shifts were observed between conventional LAESI-MS and HDX-LAESI-MS experiments of proteins under native conditions despite the short HDX timescale. Obviously, intramolecular hydrogen bonded and solvent-shielded protons are less likely to be exchanged within the timeframe of a LAESI setup. Nevertheless, comparative analysis in support of rapid protein conformation QA/QC analysis and 3D structure elucidation on MS/MS instruments equipped with ETD or ECD hardware should be all within reach.
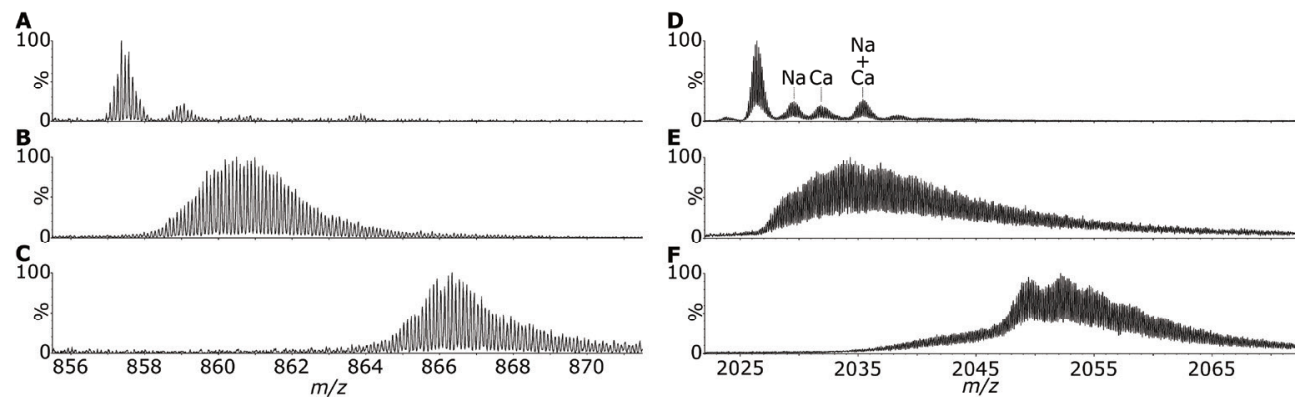

Figure 3. LAESI-MS and HDX-LAESI-MS background-subtracted mass spectra of the proteins ubiquitin (10+ charge state) and $\alpha$-lactalbumin (7+ charge state). A presents LAESI-MS of an aqueous ubiquitin solution, B presents HDXLAESI-MS of an aqueous ubiquitin solution and $\mathbf{C}$ shows LAESI-MS with ubiquitin dissolved in $95 \% \mathrm{D}_{2} \mathrm{O}$ (for $30 \mathrm{~min}$ ) and $\mathrm{D}_{2} \mathrm{O}$ as ESI solvent. Panel $\mathbf{D}$ shows the LAESI-MS mass spectrum of an aqueous $\alpha$-lactalbumin solution, $\mathbf{E}$ presents HDX-LAESI-MS of an aqueous $\alpha$-lactalbumin solution and $\mathbf{F}$ shows LAESI-MS with $\alpha$-lactalbumin dissolved in $95 \% \mathrm{D}_{2} \mathrm{O}$ (for $30 \mathrm{~min}$ ) and $\mathrm{D}_{2} \mathrm{O}$ as ESI solvent. The mass spectrum showing all detected charge states of ubiquitin is provided in Figure S5.

\section{Ambient HDX-LAESI-MS and MSI of Fruit and Tissue Slices}

An abundant class of biomolecules in fruits are oligosaccharides. Oligosaccharides contain a substantial number of exchangeable protons and are therefore an excellent class of biomolecules to evaluate ambient HDX-LAESI-MS for real biological samples. Oligosaccharides are detected with HDX-LAESI-MS in positive ion mode as sodium adducts, so theoretical $\mathrm{m} / \mathrm{z}$ values of 365.1054 for $\left[\mathrm{C}_{12} \mathrm{H}_{22} \mathrm{O}_{11}+\mathrm{Na}\right]^{+}, 527.1583$ for $\left[\mathrm{C}_{18} \mathrm{H}_{32} \mathrm{O}_{16}+\mathrm{Na}\right]^{+}$, 689.2111 for $\left[\mathrm{C}_{24} \mathrm{H}_{42} \mathrm{O}_{21}+\mathrm{Na}\right]^{+}$, etc. are expected to be observed in LAESI-MS experiments of oligosaccharides. With HDX-LAESI-MS these $m / z$ values can be shifted up to a maximum of $5+3 \times(n-1)$, in which $n$ is the number of monomers from $n=1$ onwards, resulting in maximum $m / z$ value shifts of $+8,+11,+14$, etc. for di-, tri-, and tetra saccharides, respectively. Figure 4 presents mass spectra of carbohydrates obtained with LAESI-TWIM-MS and HDX-LAESITWIM-MS from the surface of an orange slice. Figure 4A shows the $m / z$ value of 689.21 for $\left[\mathrm{C}_{24} \mathrm{H}_{42} \mathrm{O}_{21}+\mathrm{Na}\right]^{+}$, and Figure 4B depicts HDX-LAESI-TWIM-MS which clearly shows the 
expected $m / z$ shift - for $5+3 \times(4-1)$ - of up to +14 , followed by a small ${ }^{13} \mathrm{C}$ isotope peak having a similar isotope ratio as in Figure 4A (Figures S6 and S7 present similar results obtained with the other oligosaccharides). In Figure $4 \mathrm{~A} \mathrm{~m} / \mathrm{z} 689.21$ is located at the tail of an unresolved interfering background ion at $\mathrm{m} / z 689.1592$ (mass spectra of ESI solvent background and orange with ESI solvent background are shown in Figures 4C-D, respectively), therefore the mass assignment is less accurate. In HDX-LAESI-TWIM-MS, however, the exact $\mathrm{m} / \mathrm{z}$ 703.2290 of $\left[\mathrm{C}_{24} \mathrm{H}_{28} \mathrm{D}_{14} \mathrm{O}_{21}+\mathrm{Na}\right]^{+}$is clearly obtained within $10 \mathrm{ppm}$ mass accuracy. This shows that, when TWIM cannot deal with unresolved background interferences, using HDX the $\mathrm{m} / \mathrm{z}$ value can be shifted to obtain enhanced mass accuracy and sensitivity. The number of observed exchanges in the oligosaccharides is in excellent agreement with previously reported HDX-MALDI and ESI in-source HDX-MS studies of oligosaccharides. ${ }^{51,76}$ Interestingly, Liyanage et al. reported that a trisaccharide sodium adduct ion with theoretically 11 exchangeable protons does not undergo gas-phase HDX reactions, due to its highly unfavorable basicity in the gas-phase. ${ }^{77}$ The current number of observed $\mathrm{H} / \mathrm{D}$ exchanges, as with bradykinin, therefore clearly suggest that the exchange reaction occurs inside the liquid microdroplets generated by the LAESI process.

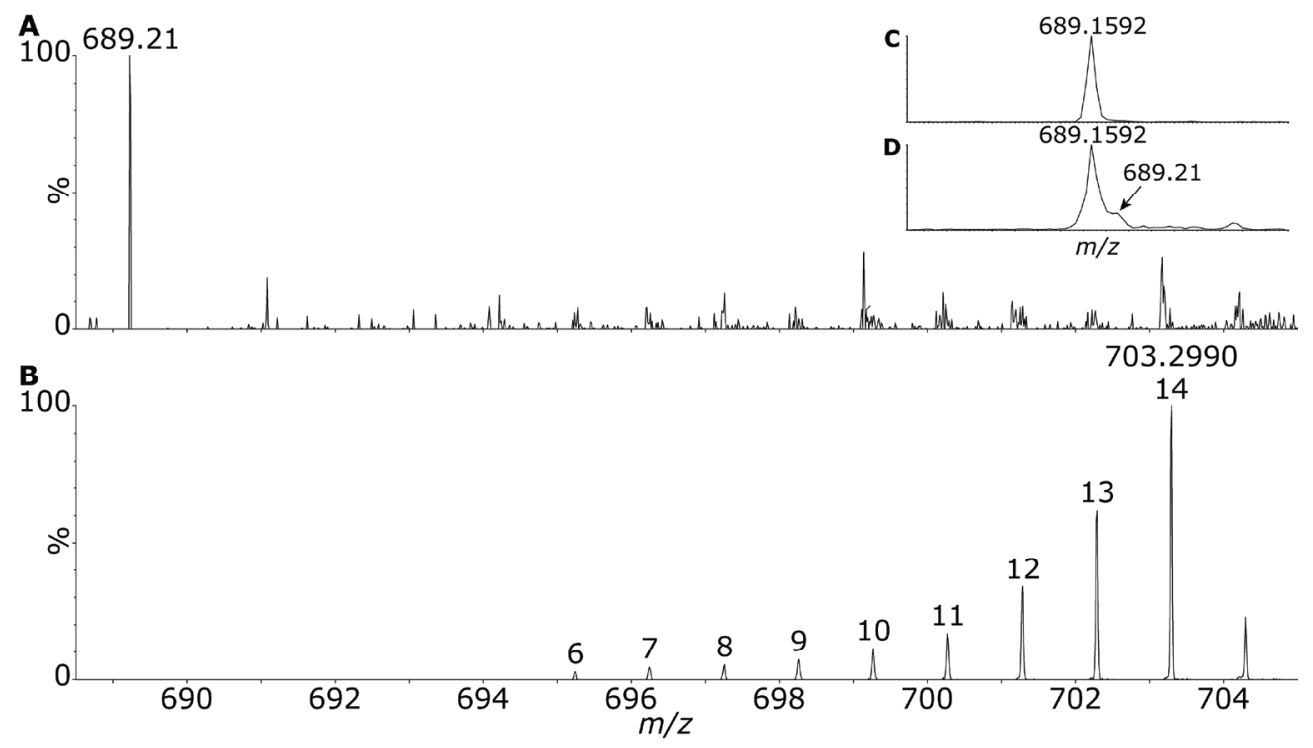

Figure 4. LAESI-TWIM-MS and HDX-LAESI-TWIM-MS of an orange slice. Both $\mathbf{A}$ and $\mathbf{B}$ show IMS drift time 85-92 bins selected and subsequently background-subtracted mass spectra of an orange obtained with A LAESI-TWIM-MS and B HDX-LAESI-TWIM-MS analysis. Additionally, insert in A: $\mathbf{C}$ shows the ESI solvent background and $\mathbf{D}$ shows the orange mass spectrum including background. Obviously the mass resolving power is insufficient to baseline separate the cationized oligosaccharide from the background interference. 
Apart from carbohydrates many other ions were detected in the orange slice such as, e.g., $m / z 175.1189$ (Figure 5A). This relates to an unexpected endogenous compound for which HDX strongly facilitated its structure elucidation. Based on the exact mass only 1 elemental composition (comprising: $\mathrm{C}_{0-20}, \mathrm{H}_{0-50}, \mathrm{O}_{0-10}, \mathrm{~N}_{0-10}, \mathrm{~S}_{0-2}, \mathrm{P}_{0-2}, \mathrm{Na}_{0-1}, \mathrm{~K}_{0-1}$ ) was obtained within 10 ppm mass accuracy: $\mathrm{C}_{6} \mathrm{H}_{15} \mathrm{~N}_{4} \mathrm{O}_{2}$, so obviously a protonated substance rather than a cationized species. A SciFinder ${ }^{\circledR}$ elemental composition search - excluding substances comprising isotopes and substances without any references - resulted in 103 chemical structures. HDX-LAESI-MS showed up to 8 exchanges to occur (Figure 5B), including the deuteronation. As a result of the 7 detected exchangeable protons for $\left[M-d_{7}+D\right]^{+}$the search was refined to only include compounds comprising 7 proton donors. As a result from the initial 103 options only 13 structures fulfil this requirement (Table S1), which includes several sets of stereoisomers. Upon excluding stereoisomers, which cannot be resolved by MS alone, 10 out of the remaining 11 substances have only 1-5 references with various origins such as, e.g., synthesis, patents, and review articles. One substance however, arginine, is obviously well documented with approximately 100,000 references and would justify a hypothesis that the unexpected ion in the orange slice belongs to arginine. Indeed, this hypothesis could be confirmed by literature. ${ }^{78-80}$ Fragmentation by MS/MS on $\mathrm{m} / \mathrm{z} 175.1189$ could experimentally verify the proposed compound, but that was beyond the scope of the present study.

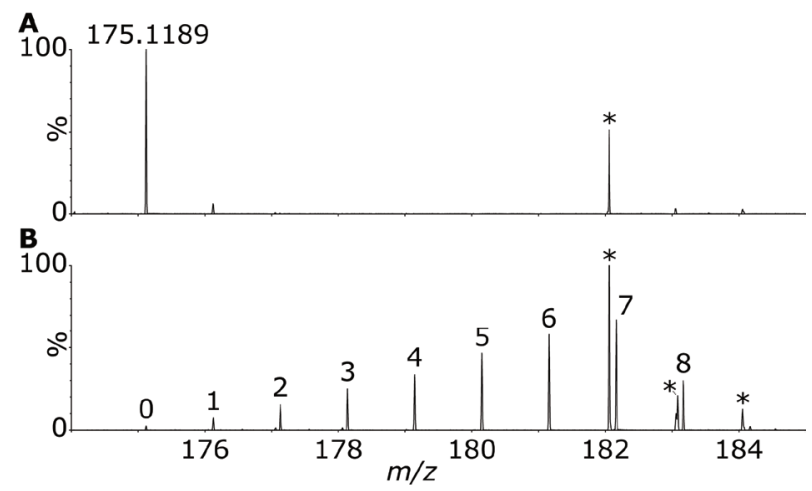

Figure 5. LAESI-MS and HDX-LAESI-MS of an orange slice. Both $\mathbf{A}$ and $\mathbf{B}$ show background-subtracted mass spectra of arginine within an orange, obtained with A LAESI-MS and B HDX-LAESI-MS analysis. The signals marked with a * belong to other unknown biomolecules.

Next, in order to show the full potential of ambient HDX surface analysis, a (HDX-) LAESI-MSI experiment was performed on the same orange slice. The laser ablation $x-y$ coordinates were used to create $2 D$ ion maps of the obtained arginine and fully $H / D$ exchanged arginine LAESI-MS signals, and both were superimposed on an optical image of the orange (Figure $6 A-B$ ). The area inside the red box was imaged and for both ions $-m / z$ 175.12 for $\left[\mathrm{M}-\mathrm{d}_{0}+\mathrm{H}\right]^{+}$and $m / z 183.17$ for $\left[\mathrm{M}-\mathrm{d}_{7}+\mathrm{D}\right]^{+}-$images were obtained from the pulp area 
in the orange slice, with a few minor artefacts - resulting from slicing the orange - on the peel. These images show LAESI-MSI and HDX-LAESI-MSI provide nicely corresponding 2D ion maps and thereby clearly demonstrate the feasibility of HDX-LAESI-MSI in real-life food samples.
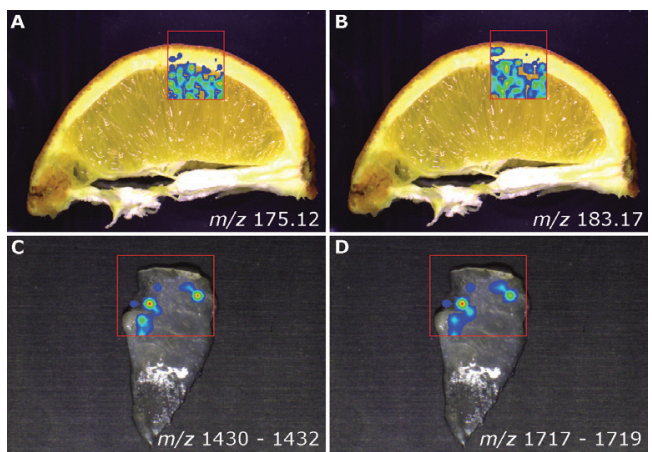

Figure 6. (HDX)-LAESI-MSI of endogenous arginine in an orange slice, and muscle tissue spiked with ubiquitin. Arginine is shown in A and $\mathbf{B}$ : A shows the LAESI-MSI 2D ion map of $m / z 175.12$ for $\left[\mathrm{M}-\mathrm{d}_{0}+\mathrm{H}\right]^{+}$whereas $\mathbf{B}$ provides the HDXLAESI-MSI 2D ion map of $m / z 183.17$ for $\left[\mathrm{M}-\mathrm{d}_{7}+\mathrm{D}\right]^{+}$. C and $\mathbf{D}$ show the HDX-LAESI-MSI 2D ion map of ubiquitin spiked muscle tissue with in $\mathbf{C}$ the ubiquitin $6+$ ion $(\mathrm{m} / \mathrm{z} 1430-1432)$ and $\mathbf{D}$ the ubiquitin $5+$ ion $($ at $m / z 1717-1719)$. The red box on the superimposed pictures marks the imaged area.

Finally, as initial feasibility experiments in order to investigate the potential of ambient MSI in future protein conformation studies, a spiked tissue slice was analyzed by HDX-LAESIMSI. Figures 6C-D show the highly similar HDX-LAESI-MSI 2D ion maps of the 6+ (Fig. 6C) and $5+$ (Fig. 6D) ubiquitin ions. In both of the imaging experiments a $m / z$ shift of approximately 6 to $30 \mathrm{Da}$ was observed, which is somewhat less than previously discussed 12 to $60 \mathrm{Da}$ for ubiquitin from an aqueous solution, likely due to matrix effects. Nevertheless, the feasibility of ambient protein HDX-LAESI-MSI is shown, and, with future hardware developments to improve the LAESI system sensitivity, eventually native protein conformations could be imaged in ambient mass spectrometry studies. 


\section{Conclusions}

We have developed a novel ambient HDX-LAESI-MS(I) approach to acquire molecular structural information for the identification and confirmation of (bio)molecules. Like all ambient MS approaches this method provides simple and rapid analysis of samples under ambient conditions but now also includes an additional amount of structural information. HDX reactions in ambient LAESI-MS were rapidly demonstrated with the model compounds melamine, monosaccharides, bradykinin, ubiquitin and $\alpha$-lactalbumin. For small molecules the maximum number of $\mathrm{H} / \mathrm{D}$ exchanges significantly reduced the number of structures in elemental composition database searches. Mass spectrometric investigation of an orange slice revealed the presence of, amongst others, oligosaccharides, and the amino acid arginine. The latter was investigated in detail, and it was proven that combining accurate mass and literature data with HDX quickly led to the unambiguous structural elucidation of that compound. Additionally, a significant increase in sensitivity was observed for oligosaccharides as a result of the HDX reaction $\mathrm{m} / \mathrm{z}$ shift away from an unresolved background interference. The method was shown viable for imaging of biological samples, using arginine in oranges, as well as spiked ubiquitin in chicken muscle tissue. As a future hardware simplification, the use of a dual- or theta ESI sprayer - one with $\mathrm{H}_{2} \mathrm{O}$ and the other with $\mathrm{D}_{2} \mathrm{O}$ - can be considered in the LAESI set-up. This HDX-LAESI-MS(I) method can be used in future ambient MS research to elucidate structures of unknown chemical entities, support rapid QA/QC of native protein conformation in production settings, potentially increase sensitivity and selectivity in LAESI-MS and possibly allow ambient MSI of different protein conformations under native conditions.

\section{Acknowledgements}

The authors are grateful to receive funding for this research from The Netherlands Organization for Scientific Research (NWO) in the framework of the Technology Area TACOAST2 of the Fund New Chemical Innovations (Project No. 053.21.111). Pepijn Beekman and Sevil Sahin are acknowledged for inspiring conversations. 


\section{References}

Bowman, A. P.; Heeren, R. M. A.; Ellis, S. R. TrAC, Trends Anal. Chem. 2018.

Lamont, L.; Eijkel, G. B.; Jones, E. A., et al. Anal. Chem. 2018, 90, 13229-13235.

Cillero-Pastor, B.; Heeren, R. M. A. J. Proteome Res. 2014, 13, 325-335.

McDonnell, L. A.; Heeren, R. M. A. Mass Spectrom. Rev. 2007, 26, 606-643.

Caprioli, R. M. Proteomics 2014, 14, 807-809.

Dettmer, K.; Aronov, P. A.; Hammock, B. D. Mass Spectrom. Rev. 2007, 26, 5178.

Wu, C.; Dill, A. L.; Eberlin, L. S., et al. Mass Spectrom. Rev. 2013, 32, 218-243.

Cha, S.; Zhang, H.; Ilarslan, H. I., et al. Plant J. 2008, 55, 348-360.

Thomas, A.; Charbonneau, J. L.; Fournaise, E.; Chaurand, P. Anal. Chem. 2012, 84, 2048-2054.

Cooks, R. G.; Ouyang, Z.; Takats, Z.; Wiseman, J. M. Science 2006, 311, 1566.

Cody, R. B.; Laramée, J. A.; Durst, H. D. Anal. Chem. 2005, 77, 2297-2302.

Venter, A.; Nefliu, M.; Cooks, R. G. TrAC, Trends Anal. Chem. 2008, 27, 284-290.

Chen, H.; Gamez, G.; Zenobi, R. J. Am. Soc. Mass Spectrom. 2009, 20, 19471963.

Perez, C. J.; Bagga, A. K.; Prova, S. S., et al. Rapid Commun. Mass Spectrom. 2018, doi.org/10.1002/rcm.8145.

Manicke, N. E.; Dill, A. L.; Ifa, D. R.; Cooks, R. G. J. Mass Spectrom. 2010, 45, 223-226.

Little, J. L.; Cleven, C. D.; Brown, S. D. J. Am. Soc. Mass Spectrom. 2011, 22, 348-359.

Cajka, T.; Fiehn, O. Methods Mol. Biol. 2017, 1609, 149-170.

Li, H.; Smith, B. K.; Márk, L., et al. Int. J. Mass Spectrom. 2015, 377, 681-689.

Bennett, R. V.; Gamage, C. M.; Galhena, A. S.; Fernández, F. M. Anal. Chem. 2014, 86, 3756-3763.

van Geenen, F. A. M. G.; Franssen, M. C. R.; Zuilhof, H.; Nielen, M. W. F. Anal. Chem. 2018, 90, 10409-10416.

Chen, H.; Cotte-Rodriguez, I.; Cooks, R. G. Chem. Commun. 2006, 597-599.

Kostyukevich, Y.; Acter, T.; Zherebker, A., et al. Mass Spectrom. Rev. 2018, 37, 811-853.

Masson, G. R.; Jenkins, M. L.; Burke, J. E. Expert Opin. Drug Discov. 2017, 12, 981-994.

Trabjerg, E.; Nazari, Z. E.; Rand, K. D. TrAC, Trends Anal. Chem. 2018, 106, 125138.

Konermann, L.; Pan, J.; Liu, Y.-H. Chem. Soc. Rev. 2011, 40, 1224-1234.

Cheng, X.; Fenselau, C. Int. J. Mass Spectrom. Ion Processes 1992, 122, 109-119.

Hunt, D. F.; McEwen, C. N.; Upham, R. A. Anal. Chem. 1972, 44, 1292-1294.

Adejare, A.; Brown, P. W. Anal. Chem. 1997, 69, 1525-1529.

Chen, H.; Zheng, J.; Zhang, X., et al. J. Mass Spectrom. 2007, 42, 1045-1056.

Vail, T. M.; Jones, P. R.; Sparkman, O. D. J. Anal. Toxicol. 2007, 31, 304-312.

Nemes, P.; Vertes, A. Anal. Chem. 2007, 79, 8098-8106.

Nemes, P.; Barton, A. A.; Li, Y.; Vertes, A. Anal. Chem. 2008, 80, 4575-4582.

Vertes, A.; Nemes, P.; Shrestha, B., et al. Appl. Phys. A 2008, 93, 885-891.

Sripadi, P.; Nazarian, J.; Hathout, Y., et al. Metabolomics 2009, 5, 263-276.

Nemes, P.; Barton, A. A.; Vertes, A. Anal. Chem. 2009, 81, 6668-6675.

Nemes, P.; Vertes, A. Methods Mol. Biol. 2010, 656, 159-171.

Nielen, M. W. F.; van Beek, T. A. Anal. Bioanal. Chem. 2014, 406, 6805-6815. van Geenen, F. A. M. G.; Franssen, M. C. R.; Schotman, A. H. M., et al. Anal. Chem. 2017, 89, 4031-4037.

Shrestha, B.; Vertes, A. Anal. Chem. 2009, 81, 8265-8271. 
Shrestha, B.; Vertes, A. J. Vis. Exp. 2010, e2144.

Shrestha, B.; Patt, J. M.; Vertes, A. Anal. Chem. 2011, 83, 2947-2955.

Kiss, A.; Smith, D. F.; Reschke, B. R., et al. Proteomics 2014, 14, 1283-1289.

Nazari, M.; Ekelöf, M.; Khodjaniyazova, S., et al. Rapid Commun. Mass Spectrom. 2017, 31, 1868-1874.

van Geenen, F. A. M. G.; Franssen, M. C. R.; Miikkulainen, V., et al. J. Am. Soc. Mass Spectrom. 2019, doi.org/10.1007/s13361-13018-12120-x.

Kostyukevich, Y.; Kononikhin, A.; Popov, I.; Nikolaev, E. Anal. Chem. 2013, 85, 5330-5334.

Katta, V.; Chait, B. T. J. Am. Chem. Soc. 1993, 115, 6317-6321.

Kim, H. J.; Liyanage, O. T.; Mulenos, M. R.; Gallagher, E. S. J. Am. Soc. Mass Spectrom. 2018, 29, 2030-2040.

Jansson, E. T.; Lai, Y.-H.; Santiago, J. G.; Zare, R. N. J. Am. Chem. Soc. 2017, 139, 6851-6854.

Nielen, M. W. F.; van de Ven, H. J. F. M. Rapid Commun. Mass Spectrom. 1996, 10, 74-81.

Kostyukevich, Y.; Kononikhin, A.; Popov, I.; Nikolaev, E. Anal. Chem. 2014, 86, 2595-2600.

Price, N. P. J. Anal. Chem. 2006, 78, 5302-5308.

Choi, S. S.; Kim, J. C. Carbohydr. Res. 2010, 345, 408-413.

Carballo, S.; Zingarello, F. A.; Maestre, S. E., et al. Int. J. Food Sci. Technol. 2014, 49, 146-152.

Zhang, Q.; Willison, L. N.; Tripathi, P., et al. Anal. Chem. 2011, 83, 7129-7136.

Pan, J.; Zhang, S.; Chou, A.; Borchers, C. H. Chem. Sci. 2016, 7, 1480-1486.

Englander, S. W.; Sosnick, T. R.; Englander, J. J.; Mayne, L. Curr. Opin. Struc. Biol. 1996, 6, 18-23.

Englander, S. W.; Mayne, L.; Bai, Y.; Sosnick, T. R. Protein Sci. 1997, 6, 11011109.

Tüchsen, E.; Woodward, C. J. Mol. Biol. 1985, 185, 421-430.

Dempsey, C. E. Biochemistry 1988, 27, 6893-6901.

Zhou, S.; Prebyl, B. S.; Cook, K. D. Anal. Chem. 2002, 74, 4885-4888.

Gimon-Kinsel, M. E.; Barbacci, D. C.; Russell, D. H. J. Mass Spectrom. 1999, 34, 124-136.

Pierson, N. A.; Chen, L.; Valentine, S. J., et al. J. Am. Chem. Soc. 2011, 133, 13810-13813.

Lee, J. K.; Kim, S.; Nam, H. G.; Zare, R. N. Proc. Natl. Acad. Sci. U.S.A 2015, 112, 3898-3903.

Freitas, M. A.; Marshall, A. G. Int. J. Mass Spectrom. 1999, 182-183, 221-231.

Mao, D.; Douglas, D. J. J. Am. Soc. Mass Spectrom. 2003, 14, 85-94.

Hilser, V. J.; Freire, E. J. Mol. Biol. 1996, 262, 756-772.

Resing, K. A.; Hoofnagle, A. N.; Ahn, N. G. J. Am. Soc. Mass Spectrom. 1999, 10, 685-702.

Brodie, N. I.; Huguet, R.; Zhang, T., et al. Anal. Chem. 2018, 90, 3079-3082.

Abzalimov, R. R.; Kaplan, D. A.; Easterling, M. L.; Kaltashov, I. A. J. Am. Soc. Mass Spectrom. 2009, 20, 1514-1517.

Pan, J.; Han, J.; Borchers, C. H.; Konermann, L. J. Am. Chem. Soc. 2009, 131, 12801-12808.

Hershko, A. Trends Biochem. Sci 1991, 16, 265-268.

Pan, J.; Wilson, D. J.; Konermann, L. Biochemistry 2005, 44, 8627-8633.

Brew, K.; Castellino, F. J.; Vanaman, T. C.; Hill, R. L. J. Biol. Chem. 1970, 245, 4570-4582.

Ebner, K. E.; Brodbeck, U. J. Dairy Sci. 1968, 51, 317-322.

Tanaka, N.; Kunugi, S. Int. J. Biol. Macromol. 1996, 18, 33-39. 
76 Kostyukevich, Y.; Kononikhin, A.; Popov, I.; Nikolaev, E. J. Mass Spectrom. 2015, 50, 1150-1156.

77 Liyanage, O. T.; Brantley, M. R.; Calixte, E. I., et al. J. Am. Soc. Mass Spectrom. 2018, doi.org/10.1007/s13361-13018-12080-13361.

78 Wang, S.; Tu, H.; Wan, J., et al. Food Chem. 2016, 199, 8-17.

79 Verma, N.; Singh, A. K.; Saini, N. Sens. Biosensing Res. 2017, 15, 41-45.

80 de Oliveira, C. R.; Carneiro, R. L.; Ferreira, A. G. Food Chem. 2014, 164, 446-453. 


\section{Supporting Information Chapter 5}

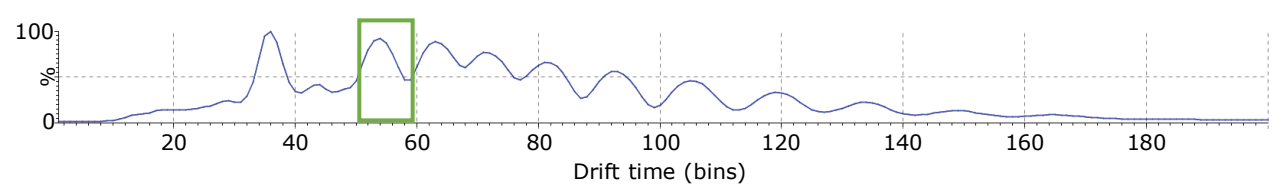

Figure S1. Drift time selection for oligosaccharides in (HDX)-LAESI-TWIM-MS. For trisaccharides drift time bins 52-60 were selected as marked by the green square.

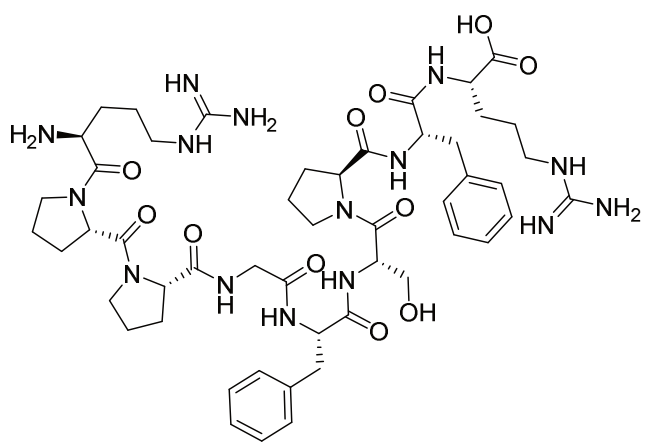

Figure S2. Chemical structure of Bradykinin. Bradykinin (Arg-Pro-Pro-Gly-Phe-Ser-Pro-Phe-Arg) has 17 exchangeable protons. 

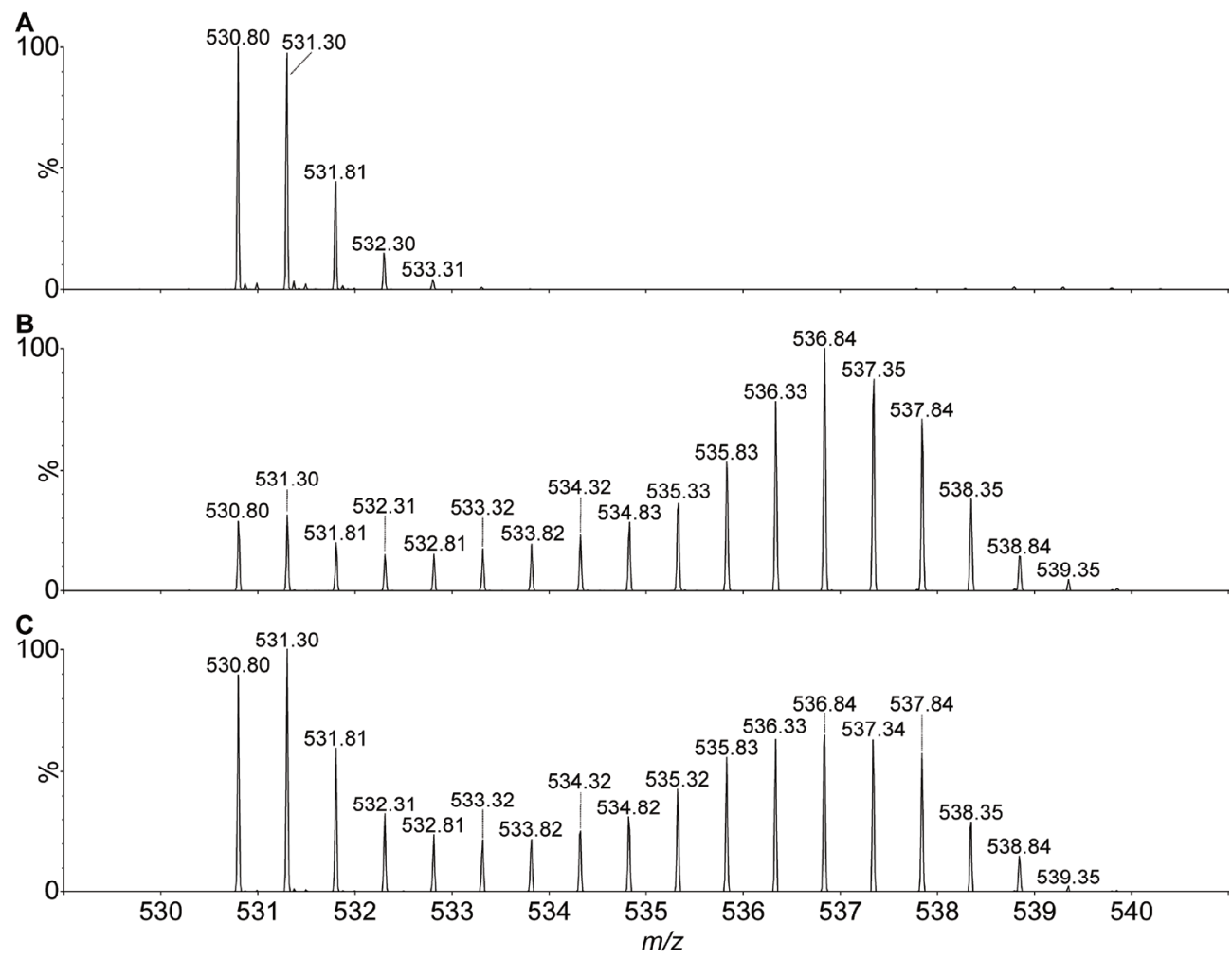

Figure S3. HDX-LAESI-MS background-subtracted mass spectra of bradykinin at various ESI solvent pH values. A ESI solvent was $\mathrm{D}_{2} \mathrm{O}+0.1 \%$ FA-D . B ESI solvent was $\mathrm{D}_{2} \mathrm{O}+10 \mathrm{mM} \mathrm{NH}_{4} \mathrm{Ac} \mathrm{pH}$. C ESI solvent was $\mathrm{D}_{2} \mathrm{O}+10 \mathrm{mM} \mathrm{NH}_{4} \mathrm{Ac}$ $\mathrm{pH} 9$. 
Chapter 5
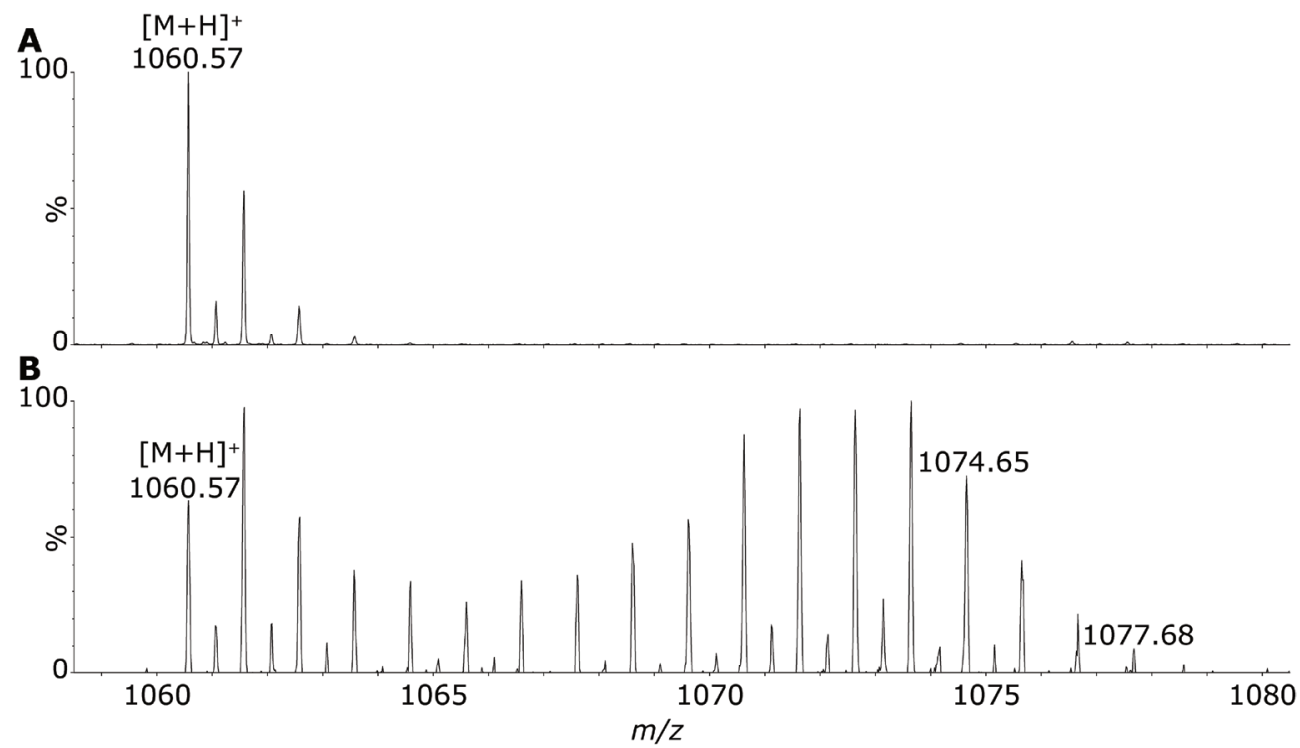

Figure S4. (HDX)-LAESI-MS background-subtracted mass spectra of Bradykinin. A LAESI of a 0.2 mM aqueous bradykinin solution (ESI solvent was $\mathrm{H}_{2} \mathrm{O}+10 \mathrm{mM} \mathrm{NH}_{4} \mathrm{Ac} \mathrm{pH}$ 7). B HDX-LAESI of a $0.2 \mathrm{mM}$ aqueous bradykinin solution (ESI solvent was $\mathrm{D}_{2} \mathrm{O}+10 \mathrm{mM} \mathrm{NH}_{4} \mathrm{Ac} \mathrm{pH}$ 7). 

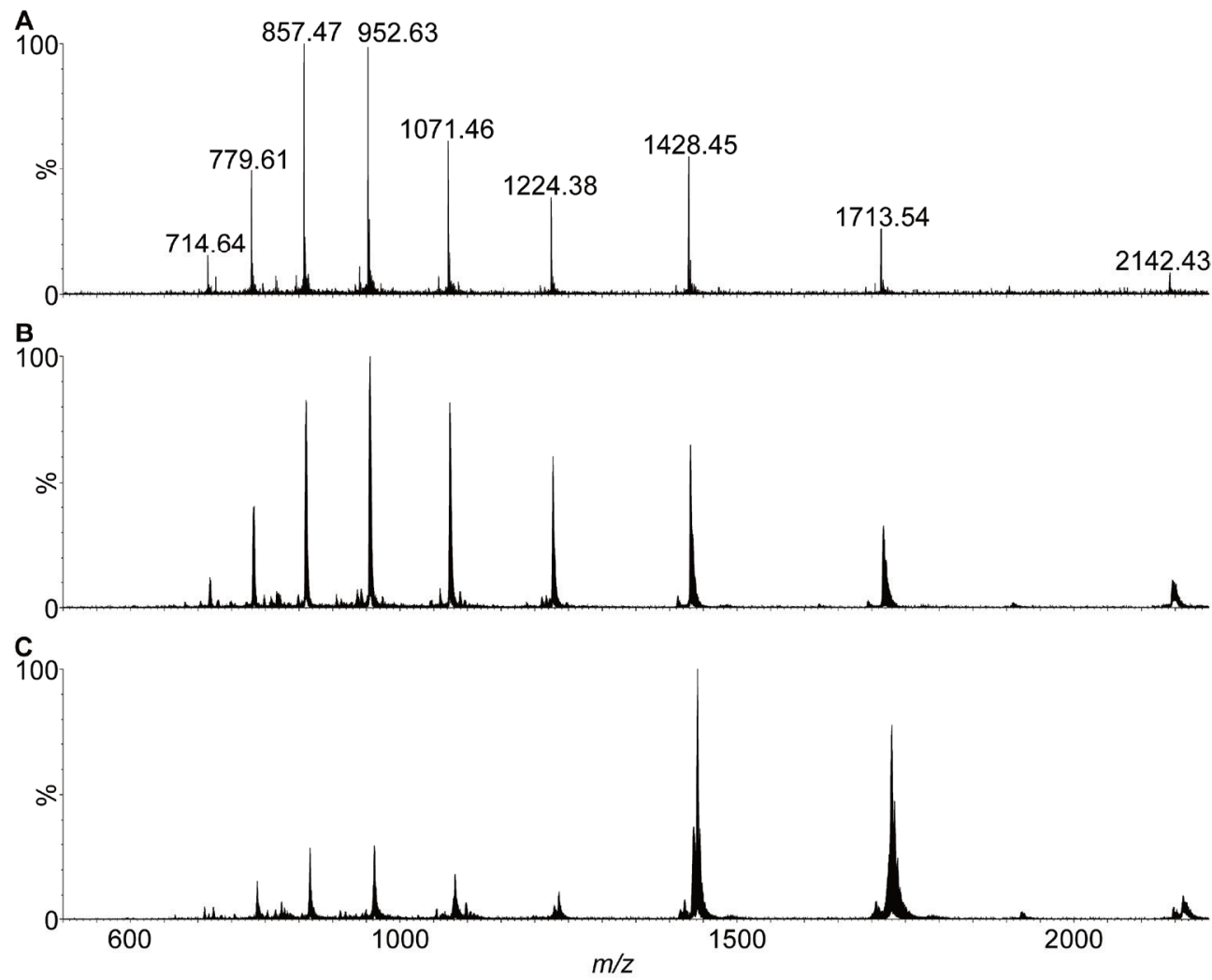

Figure S5. Ubiquitin (HDX)-LAESI-MS $\mathrm{m} / \mathrm{z}$ range 500 - 2200. A shows multiple charges in the background-subtracted LAESI-MS mass spectrum of ubiquitin with the $10+$ charge state at $m / z 857$. B presents the background-subtracted HDXLAESI-MS mass spectrum and $\mathbf{C}$ shows the LAESI-MS (ESI solvent was $\mathrm{D}_{2} \mathrm{O}$ with $10 \mathrm{mM} \mathrm{NH}_{4} \mathrm{Ac} \mathrm{pH}$ 7) backgroundsubtracted mass spectrum of a $0.2 \mathrm{mM}$ ubiquitin solution following prolonged incubation for $30 \mathrm{~min}$ in $95 \% \mathrm{D}_{2} \mathrm{O}$. 

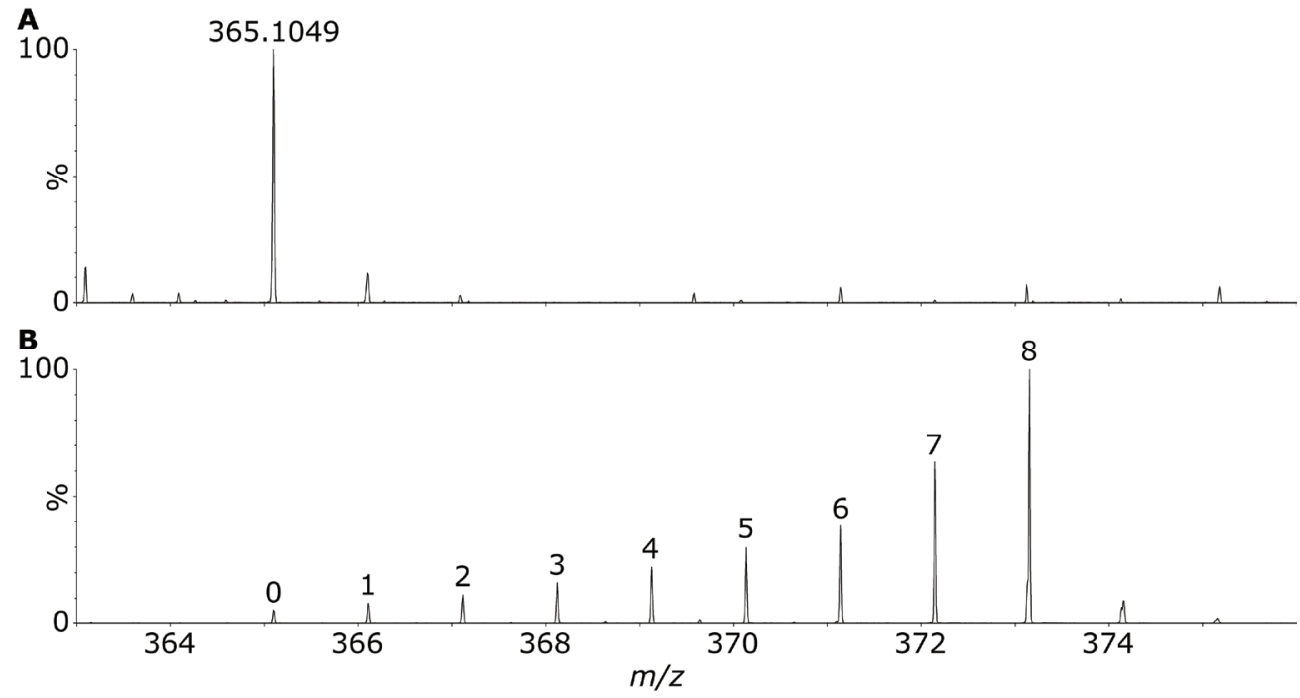

Figure S6. Disaccharides in an orange slice detected with (HDX)-LAESI-MS. Both $\mathbf{A}$ and $\mathbf{B}$ show background-subtracted mass spectra of an orange obtained with A LAESI-MS and B HDX-LAESI-MS analysis.

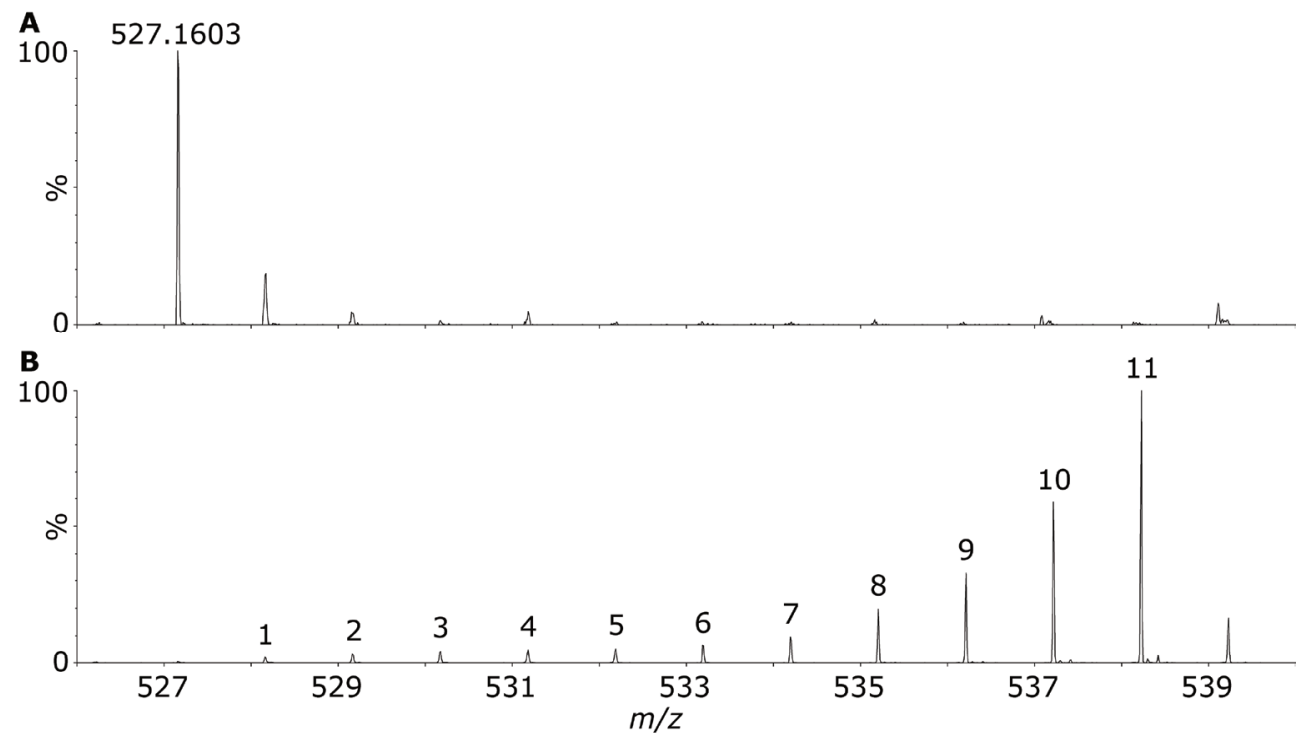

Figure S7. Oligosaccharides in an orange slice detected with (HDX)-LAESI-TWIM-MS. Both A and B show IMS drift time 52-60 bins selected and subsequently background-subtracted mass spectra of an orange slice obtained with $\mathbf{A}$ LAESI-TWIM-MS and B HDX-LAESI-TWIM-MS analysis. 


\begin{tabular}{|lc|}
\hline Substance name & $\begin{array}{c}\text { Number of } \\
\text { references }\end{array}$ \\
\hline L-Arginine & 96609 \\
\hline D-Arginine & 1319 \\
\hline Arginine & 610 \\
\hline Pentanamide, 2-amino-5-[(aminocarbonyl)amino]-, (2S)- & 5 \\
\hline Ornithine, N2-amidino- (7Cl) & 5 \\
\hline Pentanoic acid, 3-amino-5-[(aminoiminomethyl)amino]-, (3S)- & 4 \\
\hline Pentanamide, 5-[(aminoiminomethyl)amino]-2-hydroxy-, (S)- (9Cl) & 2 \\
\hline Pentanamide, 2-amino-5-[(aminocarbonyl)amino]- & 2 \\
\hline 3-Butenoic acid, 2-amino-4-(2-aminoethoxy)-, hydrazide, (3E)- & 1 \\
\hline Butanediamide, 2-[(2-aminoethyl)amino]- & 1 \\
\hline Cyclopentanecarboxylic acid, 2-hydrazinyl-2-hydroxy-, hydrazide & 1 \\
\hline Guanidine, N-(4-amino-5-hydroxy-2-pentenyl)-N'-hydroxy-, [S-(E)]- (9Cl) & 1 \\
\hline Propanediamide, 2,2-diamino-N1'-propyl-Arginine & 5 \\
\hline
\end{tabular}

Table S1. Substances retrieved from a SciFinder ${ }^{\circledR}$ database search. Search parameters were: elemental composition $\mathrm{C} 6 \mathrm{H} 14 \mathrm{~N} 4 \mathrm{O} 2$, no isotopes, only with references, and must contain 7 hydrogen donor groups. Accessed on November 30, 2018. 
Chapter 6

General Discussion and Future Perspectives 
Chapter 6 


\section{General Discussion}

Molecular imaging by mass spectrometry is gaining considerable interest since the start of this century, as can be seen from the substantial increase in MSI publications. Ambient mass spectrometry imaging was introduced to measure molecules in their native environment in real-time. Laser ablation electrospray ionization is potentially a strong candidate for the next generation of ambient mass spectrometry imaging techniques. As with most new techniques, a thorough understanding of its scope and limitations is necessary to establish its position in the rapidly changing world of contemporary MS(I) techniques. The previous chapters outlined several application areas and innovative approaches in LAESI-MS(I) analysis. In this chapter, the significant findings are discussed, including the limitations, and future perspectives.

\section{Sample Compatibility: The Effect of a Sample's Surface Composition on LAESI-MSI}

Surfaces of samples may have many different properties. Surfaces can be soft or hard, smooth or rough, liquid or solid, and may contain many different molecules involving various functional groups. The importance of surface properties in LAESI-MSI, and MSI in general, cannot be overstated as the surface forms the primary interaction between the sample and the imaging technique. In LAESI, a $2940 \mathrm{~nm}$ mid-IR laser is exploited for interaction with the sample surface in order to ablate sample material for ionization. The laser wavelength is typically absorbed by the $-\mathrm{OH}$ stretch vibration of (endogenous) molecules possessing such a moiety, like water in tissue. ${ }^{1,2}$ The laser energy is then dissipated in these molecules, leading to sample ablation and subsequent mass spectrometric detection of mostly intact analytes following the ESI ionization mechanism. ${ }^{3-6}$

Chapter 2 describes the investigation on the use of the sample's functional groups to absorb laser energy, instead of water. Apart from $-\mathrm{OH}$, also the $-\mathrm{NH}$ stretch vibration has an absorption band at $2940 \mathrm{~nm}\left(3401 \mathrm{~cm}^{-1}\right) .{ }^{7}$ Various polymer model systems comprising different functional groups, like amides and esters (molecular structures are shown in Figure 1), were examined. Indeed it was found that the laser energy was absorbed by $-\mathrm{NH}$ and $-\mathrm{OH}$ moieties, breaking the polymer backbone and leading to the MS detection of small $(<500 \mathrm{Da})$ polymer fragments. When these moieties were not present, as is the case with esters PMMA and PET, no polymer-derived signals were obtained.

Chapter 2 also established that the presence of laser absorbing compounds to dissipate the energy in a sample is a determining factor for whether LAESI is either a soft or a hard ionization technique. While irradiation of aqueous solutions leads to the generation of droplets containing intact analytes, in the absence of water even rigid structures such as polyaramid fibers were fragmented. Although the polymer materials containing $-\mathrm{NH}$ and $-\mathrm{OH}$ moieties were identified from their obtained mass spectra, the hard ionization leading to the detection of only small fragments is in contrast to one of the aims for the next generation of 
ambient MSI; image acquisition in a wide $\mathrm{m} / \mathrm{z}$ range and covering as many molecules as possible (i.e., in a complete approach).<smiles>CC(=O)NCCCCC(C)(C)C</smiles><smiles>CC(C)Nc1ccc(C(=O)Nc2ccc(C(C)(C)Br)cc2)cc1</smiles><smiles>CCc1cc(O)c(-c2nc3nc4[nH]c(C)nc4cc3[nH]2)cc1O</smiles><smiles>COC(=O)C1(C)CC2(C)CC(C)(Br)C1(C)C2(C)C</smiles><smiles>CCOC(=O)c1ccc(C(=O)OCC(C)(C)C)cc1</smiles>

Figure 1. Chemical structures of A polyamide 6, B polyaramid, C M5, D PMMA, and E PET.

The dependence of LAESI on endogenous matrix or solvent molecules to absorb and dissipate the laser energy in order to detect intact ions is not an issue for DESI, as DESI directs its solvent spray towards the surface and relies on liquid extraction of surface molecules. $^{8}$ The DESI liquid extraction mechanism means that the solubility of molecules rather than their specific electromagnetic absorption characteristics is one of the main parameters for DESI analysis. Enhancing the sensitivity of DESI analysis can simply be achieved by adjusting the solvent (composition) of the spray to match the solubility of compounds of interest. Jackson et al. investigated the analysis of polymers like poly(ethylene glycol) 200 (PEG), poly(propylene glycol) 425 (PPG), poly(methyl methacrylate) 630 (PMMA), and poly ( $\alpha$-methyl styrene) 1580 (PMS) by DESI-MS. ${ }^{9}$ This study only included relatively short chain polymers, but PMS signals were obtained for up to approximately the 14-mer. Although the average PMS polymer weight was $1580 \mathrm{Da}$, the intensities started to reduce from about the pentamer at $m / z 700$ (Figure 2). This reduction in signal intensity is likely a result of reduced solubility for longer polymer chains in DESI compatible solvents, which is limiting DESI applicability for synthetic polymer materials comprising medium and high molecular weight polymer chains. 


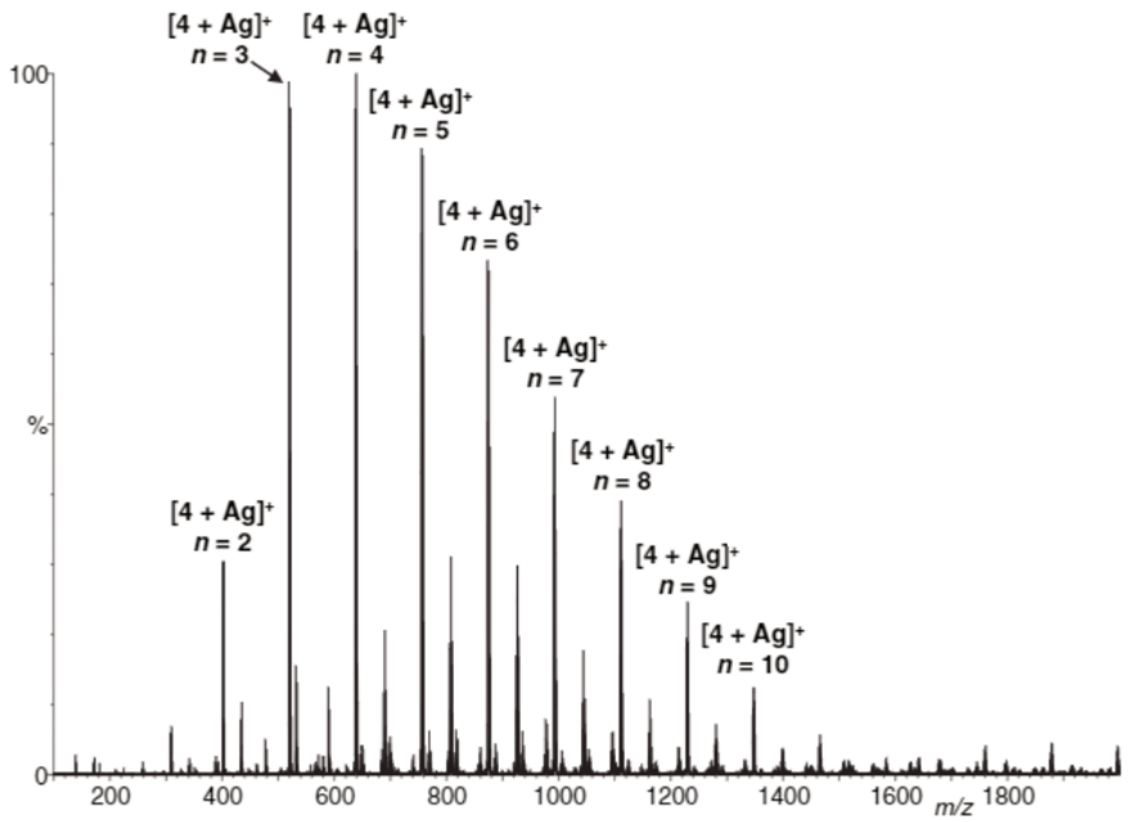

Figure 2. DESI-MS mass spectrum of PMS 1580. Image was reprinted from ${ }^{9}$, with permission from John Wiley and Sons.

For LAESI analysis, sample surfaces can also be wetted or iced by condensation of ambient water vapor on the sample. The condensation is simply achieved by decreasing the sample stage temperature before analysis, but as a non-endogenous "matrix" is added the technique should now be referred to as IR-MALDESI. The IR-MALDESI experiments in Chapter 2 show that the previously undetected aramid fiber PEG 400 based surface finish layer can be detected in such a setting. These experiments resulted in a detected polymer distribution with a mass range similar to ESI-MS analysis (Chapter 2, Figures S9B and S11C). It can be considered that when there are no suitable molecules present to absorb and dissipate laser energy, LAESI can be operated in an IR-MALDESI mode for "soft" analyte ionization in MSI. The addition of a (ice) matrix, however, removes the "ambient" aspect and might cause analyte delocalization, reducing image quality.

The LAESI dependence for resonance absorbance of laser energy was also observed for liquid samples. In chapter 5, LAESI-MS experiments in which model compounds were dissolved in either $\mathrm{H}_{2} \mathrm{O}$ or $\mathrm{D}_{2} \mathrm{O}$ were conducted. As a deuteron is ca. double the weight of a proton, the $-O D$ stretch vibration is at a completely different wavelength $\left(2625 \mathrm{~cm}^{-1}\right)$ in comparison to the $-\mathrm{OH}$ stretch vibration $\left(3530 \mathrm{~cm}^{-1}\right) .{ }^{10}$ Hence, no LAESI-MS signal from the model compounds was observed when they were dissolved in $\mathrm{D}_{2} \mathrm{O}$. Only after the addition of a few percent $\mathrm{H}_{2} \mathrm{O}$ to the solution, model compound LAESI mass spectra were obtained (Chapter 5, Figure 3). 
Apart from the presence of IR-absorbing functional groups, LAESI is suitable for the analysis of rough surfaces like tissue slices as shown in Chapter 5, Figure 6. LAESI-MSI analysis of surfaces with irregularities (in the order of a few $\mathrm{mm}$ ) is straightforward due to the (vertical) distance sample material gets ablated following laser irradiation. Non-laser based ambient MSI techniques that are capable of imaging with a spatial resolution of $<200 \mu \mathrm{m}$, such as (nano-)DESI or continuous flow liquid microjunction sampling, ${ }^{11-13}$ are much less tolerant to surface irregularities as the used capillaries have a fixed (height) position and can damage or scratch the surface.

The surface properties of samples are thus a crucial factor for LAESI-MSI feasibility, and not all sample types are compatible with LAESI-MSI analysis. In the perspective of imaging in a "complete" approach, it is of paramount importance that the analytes are embedded in a suitable compound, e.g., a solvent like water. Such an IR-absorbing compound will absorb and dissipate the laser energy, leading to mostly intact molecular ions in LAESIMSI analysis. It can be concluded that the feasibility of LAESI-MSI is highly sample dependent; however, most frequently biological (tissue) samples contain endogenous water molecules. Regarding these results, LAESI-MSI could become a useful technique for the next generation of ambient $\mathrm{MSI}$ in case of samples containing endogenous water, like tissue.

\section{Analyte Detection: The Sensitivity of LAESI-MSI}

The molecular mass range for MS imaging in a "complete" approach is an essential aspect of any ionization technique. Using LAESI, (bio)molecules have been detected directly from tissue samples, covering a wide mass range, ranging from small metabolites up to 15 kDa proteins. ${ }^{14}$ Apart from the mass range, the ability to detect molecules that are present in low concentrations is a necessity for MSI. It would be reasonable to assume that the sensitivity of molecules in LAESI analysis is related to the sensitivity that those molecules would exhibit following ESI analysis, since the same ionization mechanisms are involved. However, two other processes occur before the electrospray ionization step in LAESI: 1) laser ablation of sample material and 2) subsequent extraction of the generated sample aerosol by ESI produced microdroplets. ${ }^{1}$ As discussed in the previous paragraph, the amount of sample material ablated by the laser is strongly related to the resonance absorbance of laser energy by the sample. The laser energy in LAESI analysis is most frequently absorbed by endogenous water molecules. Apart from sample water content many more sample properties and instrumental parameters can influence the amount of sample material that gets ablated following laser irradiation. Some examples of important influencing properties or parameters are the mechanical strength of the surface (e.g., type of tissue) and the laser power (energy administered to the sample). ${ }^{15}$ General statements about LAESI sensitivity would thus be highly system, sample, and analyte dependent, and would comprise a large degree of uncertainty. 
One realistic approach to estimate LAESI sensitivity would be the isolation of the ablation and extraction steps from the final electrospray ionization step. Although analyte ESIMS sensitivity is highly complex and affected by many sample properties and instrumental parameters, the technique itself is widely known and most professionals can predict the approximate sensitivity for different classes of compounds based on a vast amount of ESI-MS literature. It would, therefore, be much more valuable to compare LAESI sensitivity to ESI sensitivity in the same hardware. The LAESI DP-1000 system is also able to operate in ESI mode, and a sample solution can be electrosprayed for MS analysis. The same sample solution can then be measured in LAESI mode, providing a clear indication of the effect of laser ablation and sample aerosol extraction on the sensitivity, relatively to ESI. This comparison experiment was performed using a PEG based lubricant solution in methanol/water 1:1. The obtained background subtracted mass spectra of both ESI-MS and LAESI-MS are presented in Figure 3. In order to roughly reach the same order of ion counts (i.e. signal intensity) with LAESI $\left(1 \times 10^{5}\right)$ as was obtained with ESI $\left(9 \times 10^{5}\right)$, the sample was required to be 1000 times more concentrated. These results indicate that (likely at best), LAESI-MS is ca. 1000 times less sensitive compared to ESI-MS. In this experiment, real-life sample properties that could potentially reduce LAESI sensitivity such as the mechanical strength of the surface, water content, analyte solubility and speed of dissolution in ESI microdroplets were not taken into account. While considering the current systems relatively poor sensitivity, it is evident that LAESI-MS requires substantial sensitivity gains if it is ever to become the next generation of ambient MSI.

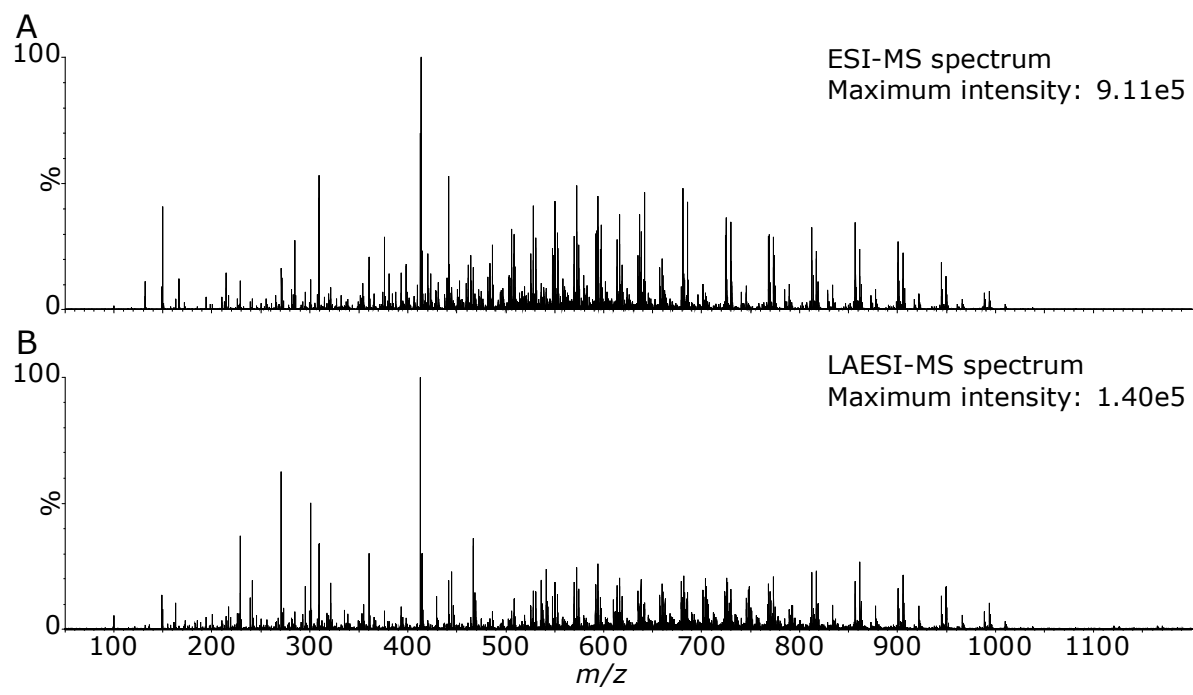

Figure 3. ESI and LAESI mass spectra of a PEG based lubricant. A shows the ESI-MS background subtracted mass spectrum of a 100.000 times diluted $\left(\mathrm{MeOH}: \mathrm{H}_{2} \mathrm{O}\right)$ PEG based lubricant. B shows the LAESI-MS background subtracted mass spectrum of the same, but only 100 times $\left(\mathrm{MeOH}: \mathrm{H}_{2} \mathrm{O}\right)$ diluted, PEG based lubricant. Both experiments were performed using the LAESI DP-1000 system hardware. 
One approach to improve LAESI-MSI sensitivity to specific -classes of- analytes is by the use of online chemical reactions, in which the analyte of interest specifically couples to a charged or easily ionisable reaction partner. This approach was demonstrated for several reactions with DESI-MS analysis, ${ }^{16,17}$ such as the reactions of ketones with hydroxylamine and cis-diols with boronic acids. ${ }^{18,19}$ In comparison with DESI, the time that sample analytes are exposed to the (electrospray) reagents in LAESI analysis is substantially reduced and is estimated to be only in the timeframe of (tens of) microseconds. ${ }^{20,21}$ Many chemical reactions require more time to take place and an increase of reaction time is thus necessary for a feasible reactive LAESI approach. Hardware improvements that increase LAESI reaction time and thereby enable reactive LAESI-MSI analysis are introduced in Chapter 3 . By the insertion of a reaction tube in-between the LAESI source and the inlet of the mass spectrometer, the reaction time could be increased by at least half a second. A reaction new to ambient MSI (an inverse electron demand Diels-Alder addition reaction) was introduced and demonstrated the effectiveness of the hardware upgrade. Although reactive LAESI-MSI sensitivity improvements following various possible chemical online reactions are highly analyte specific, require sample pre-knowledge, and restrict imaging in a complete approach, a beginning is made to improve the sensitivity of the system, as is shown in Chapter 3, Figure 6.

\section{Analyte Identification: Molecular Structure Elucidation in LAESI-MS(I)}

The primary goal of molecular imaging is to generate images of the spatial distribution of molecules. One of the significant advantages of MSI over most other molecular imaging techniques is that MSI is feasible in a non-targeted approach and without the preknowledge required for probe design and synthesis. The non-targeted imaging approach often results in the detection of a plethora of molecules in MSI studies. Unfortunately, the molecular $\mathrm{m} / \mathrm{z}$ values rather than complete molecular structures are recorded following MSI analysis. After molecular image acquisition, the $m / z$ value has to be converted to a molecular structure to study the image and understand the underlying (biological) information and effects.

Resolving molecular structures in MS is generally approached with the combination of accurate mass measurements and MS/MS or $\mathrm{MS}^{\mathrm{n}}$ fragmentation pathways. The accurate mass provides the elemental composition of the obtained $\mathrm{m} / \mathrm{z}$ value and can be used for database searches. Fragmentation pathways grant partial structural insights, aiding in the selection or confirmation of structures obtained following those database searches. Database searches often result in hundreds to thousands of possible structures and examining all of them is often a daunting task. Additionally, the complete structure including full stereochemistry cannot be elucidated by MS alone. Additional approaches to obtain some structural foreknowledge and complementary structural information are thus of high importance for ambient MSI image interpretation.

Chapter 4 describes a new approach for obtaining molecular knowledge for products of phase I metabolism oxidation reactions. These enzymatic reactions are essential for, e.g., 
the inactivation of drugs and/or the activation of prodrugs. The distribution of drugs and their metabolites in an animal or human body is an extremely important subject in pharmacology. Structure elucidation can be considerably simplified with prior knowledge on metabolite $\mathrm{m} / \mathrm{z}$ values and fragmentation patterns. $\mathrm{TiO}_{2}$ photocatalyzed oxidation of drugs can be used as a rapid non-biological method to initially investigate oxidation products for molecular information before LAESI-MSI analysis. ${ }^{22-25}$ Such an approach was reported feasible for DESI-MS. ${ }^{26}$ The $\mathrm{TiO}_{2}$ photocatalyzed oxidation products of several model compounds were similar to the DESIMS results and various in vitro studies (Chapter 4, Tables 1 and 2). It has to be noted that, although similar $\mathrm{m} / \mathrm{z}$ values and fragments were obtained, the same selectivity as enzymatic reactions cannot be reached by this approach. The obtained products are thus potential structures for the compounds that could be discovered in real-life samples. Nonetheless, we introduced a novel online time-resolved method in which sequential oxidation steps could be monitored, which helps further structural identification and provides extra information on various potential metabolites. Such prior knowledge on sample composition and target analytes is highly beneficial for targeted as well as non-targeted LAESI-MSI methods, likely resulting in a greater understanding of the biotransformation studies, despite the additional experiments required to implement this approach prior to LAESI-MSI analysis.

Chapter 5 presents a LAESI-MS(I) method to obtain structural information complementary to accurate mass and MS/MS data. In this approach, exchangeable analyte hydrogen atoms are online exchanged by heavier deuterium atoms. Each H/D exchange increases the molecular $\mathrm{m} / \mathrm{z}$ value and provides information on its functional groups (Chapter 5, Figure 1). The number of possible chemical substances that are obtained by database searches following accurate mass measurements is substantially reduced with the number of exchangeable protons included. An ambient MSI H/D exchange approach is often challenging to implement in ambient MS techniques due to (back-)exchange with ambient moisture. However, the relatively short ESI microdroplet lifetime in a LAESI-MSI setup is key to its feasibility, limiting back exchange and controlling the partial exchange of larger biomolecular conformations (due to factors as internal hydrogen bonding and solvent accessibility). A distribution of $\mathrm{H} / \mathrm{D}$ exchanged isotopes can however overlap with sample background ions, complicating data interpretation. Also the sensitivity could be reduced when the signal is spread over multiple isotopes. However, the additional complementary information obtained in an ambient $\mathrm{H} / \mathrm{D}$ exchange LAESI-MSI approach is highly valuable for both the identification as well as the confirmation of (bio)molecules and shows promise that LAESI can become the next generation of ambient MSI in the area of analyte identification. 


\section{The Future Perspectives of Ambient Laser Ablation Electrospray lonization Mass Spectrometry Imaging}

Ambient mass spectrometry imaging is being exploited for a wide variety of applications and in various research studies. New ambient MSI instrumentation is continuously being developed to better match the needs of end users, like microbiologists, pathologists, and botanists. In order to reach the end user requirements for ambient MSI, significant improvements in sensitivity, molecular mass range, and spatial resolution are vital. Ambient laser ablation electrospray ionization mass spectrometry imaging shows the potential to match those needs, but hardware updates are necessary to realize that potential. Areas of application that were previously difficult or even impossible to study can then be explored leading to a greater understanding of their topics.

The developed LAESI-MSI methods in this research contribute to a better understanding of sample compatibility, improved sensitivity, and molecular structure elucidation. One way to realize substantial sensitivity gains is by hardware improvements. Zenobi and coworkers achieved a sensitivity increase by a factor 1000 in an extractive electrospray ionization setup following the insertion of an ion funnel. ${ }^{27}$ After the laser ablation step in LAESI, the extraction of sample material by the electrospray microdroplets and subsequent ionization mechanisms are similar to the extractive electrospray ionization processes. ${ }^{28}$ Updating the LAESI-MSI hardware with the insertion of an ion funnel is therefore expected to achieve similar gains. A factor 1000 increase in sensitivity would be beneficial for many end users.

The (LAESI) laser ablation process would also require hardware updates to grant end users simple workflows that are reproducible and provide meaningful results. The laser ablation of sample material is strongly dependent on the mechanical strength of the (tissue) sample, presence of endogenous water or other IR absorbing compounds, and laser energy deposited. ${ }^{15}$ An automated system that sets the laser power for each analysis location corresponding to local water content could significantly improve the simplicity of operation and standardize the amount of ablated sample material. The system could be based on an infrared sensor that probes local water content and adjusts the laser settings accordingly. Such an approach would increase sample location-to-location as well as sample-to-sample reproducibility. Analyte quantitation will however be unlikely without an internal standard to compensate for ablation and (ESI related) ion suppression effects.

Apart from the sample properties on the efficiency of laser ablation, also the direction of sample material ejected could be influenced. A final hardware improvement in the form of an ablation cup in combination with optimized gas flow dynamics, previously demonstrated with laser ablation inductively coupled plasma mass spectrometry (Figure 4), ${ }^{29-31}$ would direct all ejected material into the continuous stream of ESI microdroplets. Such an update is expected to further improve both LAESI sensitivity and reproducibility. Although an ablation 
cup with optimized gas flow settings might be a source of laser ablation pulse-to-pulse carry over, it is likely to reduce on-sample location cross contamination as the ablated material is not polluting a wide area of the sample surface when descending back onto the surface. $A$ combination of all three hardware updates will likely establish LAESI-MSI as a unique platform for a wide variety of applications.

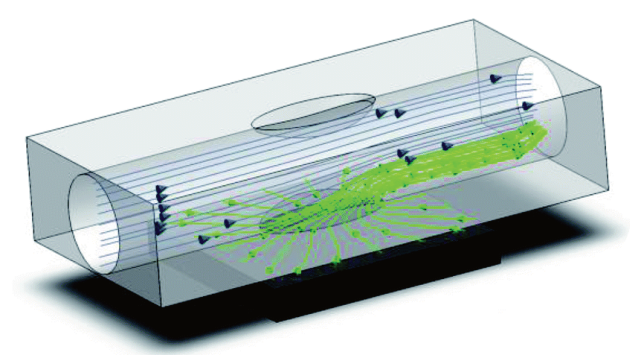

Figure 4. A potential design for a LAESI-MSI ablation cup. The blue lines show the ESI microdroplet path and the green lines shows the gas flow dynamics. Image was reprinted from ${ }^{32}$, with permission of The Royal Society of Chemistry.

An exciting area for exploration that could lead to significant biological breakthroughs is the ambient imaging of endogenous tissue proteins in their native 3D conformations, as this may shine light on their local function. In an ambient $\mathrm{H} / \mathrm{D}$ exchange LAESI-MSI approach, the ESI solvent exposed -exchangeable- protons in proteins (e.g., the protons on the outside of the 3D structure) are rapidly exchanged with deuterons from the ESI solvent. Subsequent MS/MS can then distinguish protein fragments from the inside (standard $\mathrm{m} / \mathrm{z}$ ) and the outside (increased $\mathrm{m} / \mathrm{z}$ ) of the native $3 \mathrm{D}$ protein structure. ${ }^{33}$ The $\mathrm{m} / \mathrm{z}$ value of the fragments would then provide an indication of the native protein $3 D$ structure. ${ }^{34}$ The suggested hardware updates to provide sufficient sensitivity in combination with our developed H/D exchange approach should allow ambient MSI of different protein conformations under native conditions.

Another appealing field of application would be to study the chemistry of life, which mostly takes place in small enclosed volumes such as cells or atmospheric aerosols. Macromolecular reactions, for example, are commonly studied outside the cell in uncrowded buffers; however, biological processes involving macromolecules occur in crowded environments which result in significant effects on both the rates and equilibria of reactions. ${ }^{35}$ The LAESI (electrospray) generated microdroplets are small compartments and could be a better model to study reactions that are initiated by a reagent in the ESI solvent spray, than an uncrowded buffer. The reactions of biomolecules (directly from a biological sample) with a reagent can then be studied in an ambient time-resolved reactive LAESI-MSI setup, which involves the novel reaction tube introduced in Chapter 3.

Apart from these two exciting research areas, ambient LAESI-MSI can be exploited for research in many fields, like microbiology, pathology, and botany. 


\section{Conclusions}

This research aimed to improve the capabilities and broaden the scope of laser ablation electrospray ionization mass spectrometry imaging. The main achievements that are described in this thesis offer insights on sample compatibility, hardware improvements to enable online time-resolved reactions and structure elucidation approaches. The outcome of the research chapters shows that LAESI-MS(I) is a highly versatile technique applicable to many research areas. Although the technique is highly dependent on endogenous water in samples for analysis of intact molecules, LAESI can also be exploited for the analysis and identification of (water free) polymer materials. Unfortunately, LAESI sensitivity relative to electrospray ionization is weak, and therefore the technique can currently not live up to the status of the next generation of ambient MSI. Analytes that are present in high abundance, in samples containing endogenous water (like tissue), are feasible for imaging by LAESI-MS and the technique is recommended for such samples in various research areas. For low abundance analytes, however, several hardware improvements are required to increase the sensitivity of the results substantially. When the hardware improvements are developed and implemented, the road is open for many end users in, e.g., microbiology, pathology, and botany, to make significant breakthroughs in their fields. 


\section{References}

$1 \quad$ Nemes, P.; Vertes, A. Anal. Chem. 2007, 79, 8098-8106.

2

3

4

5

6

Vertes, A.; Nemes, P.; Shrestha, B., et al. Appl. Phys. A 2008, 93, 885-891.

Nemes, P.; Barton, A. A.; Li, Y.; Vertes, A. Anal. Chem. 2008, 80, 4575-4582.

Shrestha, B.; Nemes, P.; Nazarian, J., et al. Analyst 2010, 135, 751-758.

Nemes, P.; Woods, A. S.; Vertes, A. Anal. Chem. 2010, 82, 982-988.

Nemes, P.; Vertes, A. Methods Mol. Biol. 2010, 656, 159-171.

Coates, J. In Encyclopedia of Analytical Chemistry, Meyers, R. A.; McKelvy, M. L., Eds., 2006.

Cooks, R. G.; Ouyang, Z.; Takats, Z.; Wiseman, J. M. Science 2006, 311, 1566. Jackson, A. T.; Williams, J. P.; Scrivens, J. H. Rapid Commun. Mass Spectrom. 2006, 20, 2717-2727.

Bonner, O. D.; Curry, J. D. Infrared Phys. 1970, 10, 91-94.

Wiseman, J. M.; Ifa, D. R.; Song, Q.; Cooks, R. G. Angew. Chem. Int. Ed. 2006, 45, 7188-7192.

Roach, P. J.; Laskin, J.; Laskin, A. Analyst 2010, 135, 2233-2236.

Griffiths, R. L.; Randall, E. C.; Race, A. M., et al. Anal. Chem. 2017, 89, 56835687.

Kiss, A.; Smith, D. F.; Reschke, B. R., et al. Proteomics 2014, 14, 1283-1289.

Apitz, I.; Vogel, A. Appl. Phys. A 2005, 81, 329-338.

Cotte-Rodríguez, I.; Takáts, Z.; Talaty, N., et al. Anal. Chem. 2005, 77, 67556764.

Takáts, Z.; Cotte-Rodriguez, I.; Talaty, N., et al. Chem. Commun. 2005, 19501952.

Huang, G.; Chen, H.; Zhang, X., et al. Anal. Chem. 2007, 79, 8327-8332.

Zhang, Y.; Chen, H. Int. J. Mass Spectrom. 2010, 289, 98-107.

Lee, J. K.; Kim, S.; Nam, H. G.; Zare, R. N. Proc. Natl. Acad. Sci. U.S.A 2015, 112, 3898-3903.

van Geenen, F. A. M. G.; Franssen, M. C. R.; Zuilhof, H.; Nielen, M. W. F. Anal. Chem. 2018, 90, 10409-10416.

Ruokolainen, M.; Gul, T.; Permentier, H., et al. Eur. J. Pharm. Sci. 2016, 83, 3644.

Ruokolainen, M.; Ollikainen, E.; Sikanen, T., et al. J. Am. Chem. Soc. 2016, 138, 7452-7455.

Ruokolainen, M.; Valkonen, M.; Sikanen, T., et al. Eur. J. Pharm. Sci. 2014, 65, 45-55.

Nissilä, T.; Sainiemi, L.; Karikko, M.-M., et al. Lab Chip 2011, 11, 1470-1476.

Ruokolainen, M.; Miikkulainen, V.; Ritala, M., et al. Anal. Chem. 2017, 89, 1121411218.

Meier, L.; Berchtold, C.; Schmid, S.; Zenobi, R. Anal. Chem. 2012, 84, 2076-2080.

Chen, H.; Venter, A.; Cooks, R. G. Chem. Commun. 2006, 2042-2044.

Wang, H. A. O.; Grolimund, D.; Giesen, C., et al. Anal. Chem. 2013, 85, 1010710116.

Douglas, D. N.; Managh, A. J.; Reid, H. J.; Sharp, B. L. Anal. Chem. 2015, 87, 11285-11294.

van Malderen, S. J. M.; van Elteren, J. T.; Vanhaecke, F. J. Anal. At. Spectrom. 2015, 30, 119-125.

van Malderen, S. J. M.; Managh, A. J.; Sharp, B. L.; Vanhaecke, F. J. Anal. At. Spectrom. 2016, 31, 423-439.

Pan, J.; Han, J.; Borchers, C. H. Int. J. Mass Spectrom. 2012, 325-327, 130-138. Kostyukevich, Y.; Shulga, A. A.; Kononikhin, A., et al. Sci. Rep. 2017, 7, 6176.

Rivas, G.; Minton, A. P. Trends Biochem. Sci 2016, 41, 970-981. 
Summary 
Several molecular imaging techniques are available to study and understand biological objects, like positron-emission tomography, fluorescence, and magnetic resonance imaging. These techniques often require chemical probes and image in a targeted approach. As many biological questions can only be answered in a systems approach, molecular imaging methods that can simultaneously measure many molecules are desired. Mass spectrometry imaging (MSI) is capable of measuring many molecules simultaneously without the use of chemical probes. MSI experiments often require sample stage vacuum conditions and extensive sample pretreatment such as matrix application. Vacuum conditions can disrupt or damage biological samples, and sample pretreatment prevents real-time analyses and can cause analyte losses, analyte delocalization and denaturation of proteins. Ambient ionization was introduced to measure samples under ambient conditions without any sample pretreatment, and ambient MSI followed rapidly after that. A next generation of ambient MSI techniques is desired to improve its sensitivity, molecular mass range, and spatial resolution. This work aims to improve the capabilities and broaden the scope of laser ablation electrospray ionization (LAESI) MSI.

In order to broaden the scope and increase the understanding of ambient LAESI$\mathrm{MS}(\mathrm{I})$, polymer materials and synthetic fibers were investigated. The direct analysis of synthetic fibers under ambient conditions is highly desired to identify the polymer, the finishes applied and irregularities that may compromise its performance and value. In Chapter 2 LAESI ion mobility MS was used for the analysis of synthetic polymers and fibers. The key to this analysis was the absorption of laser light by aliphatic and aromatic nitrogen functionalities in the polymers. Analysis of polyamide (PA) 6, 46, 66, and 12 pellets and PA 6, 66, polyaramid and M5 fibers yielded characteristic fragment ions, enabling their unambiguous identification. Synthetic fibers are, in addition, commonly covered with a surface layer for improved adhesion and processing. The same setup, but operated in a transient infrared matrix-assisted laser desorption electrospray ionization mode, allowed the detailed characterization of the fiber finish layer and the underlying polymer. Differences in finish layer distribution may cause variations in local properties of synthetic fibers. In Chapter 2, also the feasibility of mass spectrometry imaging (MSI) of the distribution of a finish layer on the synthetic fiber and the successful detection of local surface defects was shown.

Reactions in confined compartments like charged microdroplets are of increasing interest, notably because of their substantially increased reaction rates. When combined with ambient MS, reactions in charged microdroplets can be used to improve the detection of analytes or to study the molecular details of the reactions in real time. In Chapter 3, we introduce a reactive LAESI time-resolved MS method to perform and study reactions in charged microdroplets. This approach was demonstrated with so-called click chemistry reactions between substituted tetrazines and a strained alkyne or alkene. Click reactions are high-yielding reactions with a high atom efficiency. Although click reactions are typically at least moderately fast, in a reactive LAESI approach a substantial increase of reaction time is 
required for these reactions to occur. This increase was achieved using microdroplet chemistry and followed by MS using the insertion of a reaction tube between the LAESI source and the MS inlet, leading to near complete conversions due to significantly extended microdroplet lifetime. This novel approach allowed for the collection of kinetic data for a model click reaction and showed in addition excellent instrument stability, improved sensitivity, and applicability to other click reactions. In Chapter 3, reactive LAESI was also demonstrated in a mass spectrometry imaging setting to show its feasibility in future imaging experiments.

In drug discovery it is important to identify phase I metabolic modifications as early as possible to screen for inactivation of drugs and/or activation of prodrugs. As the major class of reactions in phase I metabolism are oxidation reactions, oxidation of drugs with $\mathrm{TiO}_{2}$ photocatalysis can be used as a simple non-biological method to initially eliminate (pro)drug candidates with an undesired phase I oxidation metabolism. Analysis of reaction products is commonly achieved with mass spectrometry coupled to chromatography. However, sample throughput can be substantially increased by eliminating pretreatment steps and exploiting the potential of ambient MS. Furthermore, online monitoring of reactions in a time-resolved way would identify sequential modification steps. In Chapter 4 we introduce a novel (timeresolved) $\mathrm{TiO}_{2}$-photocatalysis LAESI-MS method for the analysis of drug candidates. This method was proven to be compatible with both $\mathrm{TiO}_{2}$-coated glass slides as well as solutions containing suspended $\mathrm{TiO}_{2}$ nanoparticles, and the results were in excellent agreement with studies on biological oxidation of several drugs. Additionally, a time-resolved LAESI-MS setup was developed and results for verapamil showed excellent analytical stability for online photocatalyzed oxidation reactions within the set-up up to at least one hour.

Identification and confirmation of (bio)chemical entities in ambient MS mostly involves accurate mass determination, often in combination with MS/MS work flows. However, an accurate mass only provides the elemental composition of the (bio)molecule, still resulting in numerous possible structures. MS/MS procedures are often insufficient in differentiating between the hundreds possible candidate substances in database searches. Obtaining additional information and thereby improving structural assignment as well as reducing the vast number of possible candidates is thus of high importance in any ambient MS(I) study. In Chapter 5 we present an ambient hydrogen/deuterium exchange (HDX) LAESI-MS method for structure elucidation and confirmation of (bio)molecules. The concept was demonstrated with small molecules, peptides, and proteins. Moreover, the same approach could be applied to $\mathrm{MSI}$ as shown by the ambient MSI of arginine and oligosaccharides on an orange slice. Eventually, this approach will allow spatially resolved MSI of different protein conformers and may have a major impact in the life sciences.

The main achievements that are described in this thesis offer insights on sample compatibility, hardware improvements to enable online time-resolved reactions and structure elucidation approaches. The outcome of the research chapters shows that LAESI-MS(I) is a highly versatile technique applicable to many research areas. Although the technique is highly 
dependent on endogenous water in samples for analysis of intact molecules, LAESI can also be exploited for the analysis and identification of (water free) polymer materials. Unfortunately, LAESI sensitivity relative to electrospray ionization is weak, and therefore the technique can currently not live up to the status of the next generation of ambient MSI. Analytes that are present in high abundance are feasible for imaging by LAESI-MS. For low abundance analytes, however, several hardware improvements are required to substantially increase the sensitivity of the results. When the hardware improvements are developed and implemented, the road is open for many end users in, e.g., microbiology, pathology, and botany, to make significant breakthroughs in their fields. 
Summary 


\section{Acknowledgements}

The work presented in this thesis would not be possible without the beautiful people surrounding me. Indeed, I could not have done this by myself, and I want to thank everyone for his or her support. Support is a word with so many meanings that I like to elaborate on it; support is to give encouragement to someone, to provide the right conditions to someone, or to help someone in an emotional or practical way, all because you want him to succeed. I am grateful to everyone who supported me, thank you!

I want to thank my supervisors, Michel Nielen, Maurice Franssen and Han Zuilhof. You gave the opportunity to start my $\mathrm{PhD}$ and always provided the conditions for me to succeed. Han, thank you for accepting me into your department, all the good moments during my stay, the great $\mathrm{PhD}$ trips, and for given support when needed. Maurice, you have a great personality, and I feel you managed to take some of my sharp edges away (but not my dialect!). You were always there for me, regardless if I was enthusiastic or feeling down. Thank you for becoming my supervisor during my $\mathrm{PhD}$ and all the moments we shared. Michel, in life there are only a few people someone truly looks up to, and you are one of these few people to me. You are much more than a supervisor; you are a mentor that always wants the best for his students and gets the best out of them. You prioritize the needs of your students above all and are always open to provide advice, on a professional as well as a personal level. I feel blessed for the years I could learn from you and work with you, thank you so much for everything.

I would also like to thank all my colleagues from ORC for the great atmosphere, support and fun activities. I consider ORC as a great environment to do a $\mathrm{PhD}$, and this is primarily due to all of you. I want to thank the technical staff and the secretaries; each of you is a great person that makes the life of a PhD student so much easier! I thank all staff members for the pleasant chats, gained insights, and the organization of lectures and events for us to attend. I also like to thank all PhD students, mainly for being a PhD student with me: for being part of a team that understands each other and enjoys the company of all. Many of you have become my friend! I want to share my appreciation for the input of my students, Martine, Larissa, Vincent, and you too a bit Anouk. I am also grateful for the input and support of my project partners and collaborators; you made this project possible and were always supporting our work!

As you might have noticed, I do not like to list out many names. However, I feel to mention a few persons personally. Frank Claassen, you helped me a lot during my $\mathrm{PhD}$ in so many ways. Thank you for all the help and every good moment (with a good glass of whisky). Wilco Duvivier and Bas van den Berg, thank you for helping to find my way around in Wageningen. Wilco, you helped me a lot as a fellow analytical chemist, and I enjoyed being your paranimph. Bas, you became my first friend in Wageningen and I always enjoy spending my time with 
you, bro! Esther van Andel, Andrada But, Alexandre Villela, Jorick Bruins, Satesh Gangarapu, Milou Santbergen, Alyssa van den Boom, and Alice Guarneri; thanks for all the fun moments, great chats, and great songs! Pepijn "super saiyan" Beekman and Sevil "jigglypuff" Sahin, you both are entirely awesome and have wonderful personalities. I am grateful for the countless moments we shared, and I am most happy to have you on my side during my public defense.

Finally, I want to thank my friends and family. You encouraged me to succeed, understood my time-restraints, and helped me so much in an emotional way. I am certain this thesis would not be here without your support and understanding. Thank you all so much for being a part of my life. I am grateful for every little moment with you!

Rose, I know you for so many years that you feel like my sister and I feel blessed my brother one day came home with you. During my PhD you brought Bo into this world, she is truly a wonderful girl, and I am filled with happiness when she is around. Michel, if I had the choice to choose a brother out of anyone in this world, I would want you. You are simply the best brother I can imagine. Mom, you always care for me in so many ways only a mother can, and dad, you are always looking out for me and encouraging me all the way. You provided me a home, food, love and the opportunity to grow. Mom and dad, these are the things that parents are supposed to do for their children, but I do not think that anyone could have done it better. I have never said thank you for that, so I like to take this moment to thank you for being my parents. Mom, dad, Michel, Rose, and Bo, you all show the true meaning of the word family, and I am so grateful to be part of that. I love you all.

Fred 


\section{Curriculum Vitae}

Freddie Antonius Martinus Godefridus van Geenen was born on the $22^{\text {nd }}$ of November 1984 in Waalwijk, The Netherlands. After completing high school, he studied Chemistry at Radius College. He thereafter worked for 1.5 year as applied analytical chemist at Sachem Europe before deciding to pursue a Bachelor's degree in Chemistry at Avans Hogeschool. This program included a 6-month internship in which he developed a liquid chromatographyfluorescence method for the detection of

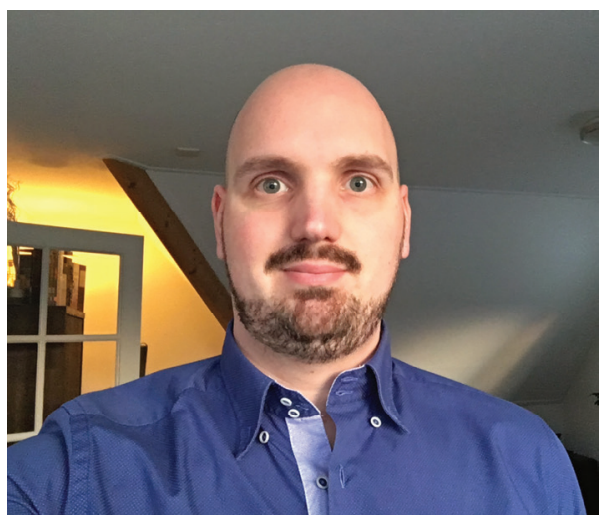
estradiol in human plasma. He then worked 6 months at Avans Hogeschool where he developed a liquid chromatography-mass spectrometry method for the detection of testosterone in human serum. During these experiences, he developed a great interest in analytical sciences and decided to take the Analytical Sciences master program at Vrije Universiteit Amsterdam. He did his research project in the bioanalysis group at Wil Research where he studied the automation of sample pre-treatment techniques for the detection of small molecules in plasma. After his MSc, he returned to Avans Hogeschool for 8 months to work on a metabolomics approach for urine metabolites by hydrophilic interaction chromatographymass spectrometry and principal component analysis. In November 2014 he started his PhD at the Laboratory of Organic Chemistry at Wageningen University under the supervision of Prof. Dr. Michel Nielen, Prof Dr. Han Zuilhof, and Dr. Maurice Franssen. The results obtained in the period 2014-2018 are described in this thesis. Freddie currently works as a post-doctoral researcher in the molecular structure and dynamics group at Radboud University. 


\section{List of Publications}

Fred A. M. G. van Geenen, Maurice C. R. Franssen, Anton H. M. Schotman, Han Zuilhof and Michel W. F. Nielen. Ambient Characterization of Synthetic Fibers by Laser Ablation Electrospray lonization Mass Spectrometry. Analytical Chemistry, 2017, 89, 4031-4037.

Fred A. M. G. van Geenen, Maurice C. R. Franssen, Han Zuilhof and Michel W. F. Nielen. Reactive Laser Ablation Electrospray Ionization Time-Resolved Mass Spectrometry of Click Reactions. Analytical Chemistry, 2018, 90, 10409-10416.

Fred A. M. G. van Geenen, Maurice C. R. Franssen, Ville Miikkulainen, Mikko Ritala, Han Zuilhof, Risto Kostiainen and Michel W. F. Nielen. $\mathrm{TiO}_{2}$ Photocatalyzed Oxidation of Drugs Studied by Laser Ablation Electrospray Ionization Mass Spectrometry. Journal of The American Society for Mass Spectrometry, 2019, 30, 639-646.

Fred A. M. G. van Geenen, Frank W. Claassen, Maurice C. R. Franssen, Han Zuilhof and Michel W. F. Nielen. Laser Ablation Electrospray Ionization Hydrogen/Deuterium Exchange for Structure Elucidation in Ambient Mass Spectrometry Imaging. Revisions submitted.

\section{Conference publications}

Fred A. M. G. van Geenen, Maurice C. R. Franssen, Han Zuilhof and Michel W. F. Nielen. Characterization of Synthetic Fibers and their Finish Layer by LAESI - MS. Proceedings of the $65^{\text {th }}$ ASMS Conference on Mass Spectrometry and Allied Topics, Indianapolis, Indiana, June 4-8, 2017.

Fred van Geenen, Satesh Gangarapu, Maurice Franssen, Michel W. F. Nielen and Han Zuilhof. Kinetics of a Click Reaction in Charged Microdroplets with Time-Resolved Reactive Laser Ablation Electrospray Ionization Mass Spectrometry. Abstract Book of the $22^{\text {nd }}$ International Mass Spectrometry Conference, Florence, Italy, August 26-31, 2018. 


\section{Overview of Completed Training Activities}

\section{General courses}

Interpersonal Communication (WGS), 2018

Effective behavior in your professional surroundings (WGS), 2018

Applied Statistics (VLAG), 2016

Chemometrics (VLAG), 2016

Efficient Writing Strategies (WGS), 2015

Presenting with Impact (WGS), 2016

Scientific Artwork with Photoshop and Illustrator (WUR Library), 2016

Brain Training (WGS), 2016

PhD Week (VLAG), 2015

\section{Discipline specific activities}

MALDI Imaging Mass Spectrometry: Basic Tools and Techniques (ASMS), Indianapolis, Indiana, USA, 2017

Ion Mobility Training (Waters), Wageningen, The Netherlands, 2015

Advanced Chemistry (VLAG), Wageningen, The Netherlands, 2014-2017

Multimodal Molecular Imaging (TI-COAST), Maastricht, The Netherlands, 2017

*65 ${ }^{\text {th }}$ ASMS Conference (ASMS), Indianapolis, Indiana, USA, 2017

*22 ${ }^{\text {nd }}$ IMSC Conference (IMSC), Florence, Italy, 2018

${ }^{*} 6^{\text {th }}$ CHAINS Conference (NWO), Veldhoven, The Netherlands, 2018

*FAST Conference (TI-COAST), Veldhoven, The Netherlands, 2018

*NVMS Conference (NVMS), Amsterdam, The Netherlands, 2017

${ }^{*}$ FAST Conference (TI-COAST), Veldhoven, The Netherlands, 2017

${ }^{*}$ TI-COAST Program Meeting (TI-COAST), Amersfoort, The Netherlands, 2016

$3^{\text {rd }}$ NVMS-BSMS Conference (NVMS-BSMS), Rolduc, The Netherlands, 2016

$3^{\text {rd }}$ CHAINS Conference (NWO), Veldhoven, The Netherlands, 2015

$4^{\text {th }}$ CHAINS Conference (NWO), Veldhoven, The Netherlands, 2016

NVMS Conference (NVMS), Delft, The Netherlands, 2017

NVMS Conference (NVMS), Nieuwegein, The Netherlands, 2018

${ }^{*}$ Represents oral presentations. Posters have been presented at the other conferences.

\section{Optional activities}

Chair of Organizing Committee PhD trip to Denmark and Sweden (ORC), 2017

Preparation of PhD Research Proposal (ORC), 2014

$\mathrm{PhD}$ trip to Denmark and Sweden (ORC), 2017 
PhD trip to Canada (ORC), 2015

Colloquia (ORC), 2014-18

Project Meetings (ORC), 2014-18

\section{Educational activities}

Analytical methods in organic chemistry, 2015-18

Organic chemistry 2, 2015

Supervision of BSc thesis students, 2016-18 
The research presented in this thesis was financially supported by The Netherlands Organization for Scientific Research (NWO) in the framework of the Technology Area TACOAST2 of the Fund New Chemical Innovations (Project No. 053.21.111).

Financial support from Wageningen University for printing this thesis is gratefully acknowledged. 

